University of Louisville

ThinkIR: The University of Louisville's Institutional Repository

Electronic Theses and Dissertations

$12-2016$

\title{
The role of nicotine, a7 nicotinic acetylcholine receptors and extracellular matrix remodeling in pulmonary fibrosis.
}

\author{
Glenn Ward Vicary \\ University of Louisville
}

Follow this and additional works at: https://ir.library.louisville.edu/etd

Part of the Animal Diseases Commons, Biological Phenomena, Cell Phenomena, and Immunity Commons, Chemical and Pharmacologic Phenomena Commons, Circulatory and Respiratory Physiology Commons, Investigative Techniques Commons, Medical Pharmacology Commons, Medical Toxicology Commons, Medicinal and Pharmaceutical Chemistry Commons, Organic Chemicals Commons, Other Pharmacy and Pharmaceutical Sciences Commons, Pulmonology Commons, Respiratory Tract Diseases Commons, and the Translational Medical Research Commons

\section{Recommended Citation}

Vicary, Glenn Ward, "The role of nicotine, a7 nicotinic acetylcholine receptors and extracellular matrix remodeling in pulmonary fibrosis." (2016). Electronic Theses and Dissertations. Paper 2589.

https://doi.org/10.18297/etd/2589

This Doctoral Dissertation is brought to you for free and open access by ThinkIR: The University of Louisville's Institutional Repository. It has been accepted for inclusion in Electronic Theses and Dissertations by an authorized administrator of ThinkIR: The University of Louisville's Institutional Repository. This title appears here courtesy of the author, who has retained all other copyrights. For more information, please contact thinkir@louisville.edu. 


\title{
THE ROLE OF NICOTINE, $\alpha 7$ NICOTINIC ACETYLCHOLINE RECEPTORS
} AND EXTRACELLULAR MATRIX REMODELING IN PULMONARY FIBROSIS

By

\author{
Glenn Ward Vicary \\ B.A., Tusculum College, 2010 \\ M.S., University of Louisville, 2014

\begin{abstract}
A Dissertation
Submitted to the Faculty of the

School of Medicine of the University of Louisville

in Partial Fulfillment of the Requirements for the Degree of
\end{abstract}

Doctor of Philosophy

in

Pharmacology and Toxicology

Department of Pharmacology and Toxicology

University of Louisville

Louisville, Kentucky

December, 2016 
Copyright 2016 by Glenn Ward Vicary

All Rights Reserved 

THE ROLE OF NICOTINE, $\alpha 7$ NICOTINIC ACETYLCHOLINE RECEPTORS AND EXTRACELLULAR MATRIX REMODELING IN PULMONARY FIBROSIS

\section{By}

Glenn Ward Vicary

B.A., Tusculum College, 2010

M.S., University of Louisville, 2014

\section{A Dissertation Approved on}

November $22^{\text {nd }}, 2016$

by the following Dissertation Committee:

Jesse Roman, M.D. Dissertation Director

Gavin E. Arteel, Ph.D. Committee Member

Shirish Barve, Ph.D. Committee Member

David A. Scott, Ph.D. Committee Member

Patricia Soucy, Ph.D. Committee Member 


\section{DEDICATION}

I dedicate this dissertation to my parents, Thomas and Joan Vicary, for their support to chase my goals of earning a doctorate in pharmacology and toxicology. My parents have always practiced tough love, allowing me the freedom to make my own successes and failures. The past six years have been taxing on them as they have seen me struggle with my research and becoming a father. No matter how bad the day, even if they knew nothing about the science, they were there to listen and motivate me to proceed on. I will never be able to thank them enough for being there, but hopefully I can help get you into a nice retirement home.

"At the end of the day, the most overwhelming key to a child's success is the positive involvement of parents." - Jane D. Hull 


\section{ACKNOWLEDGEMENTS}

Earning a doctorate is like summiting Mount Everest. Very few people attempt the journey, even fewer achieve success. When they do, it is not accomplished alone, but with the help of many. I have several friends who have meant more to me during this degree than I could ever put into words.

The mental stress of earning a doctorate is just as taxing as the long hours. Being able to laugh with "The Best Friend's Club" consisting of Dr. Alex Bajorek, Dr. Daniel Fioret, Mason Griffin, Dr. Erik Korte and Grayson Sharpe always provided much needed comic relief.

Brett and Lauren Bearfield whom I consider my Louisville adoptive parents. Although I may yell at them as their coach on the kickball diamond, they have been important pillars in my life. Brett helped me to discover running, often in some ridiculous mud run. Lauren educated me about the great city of Detroit. Of all my memories, being the "best person" in their wedding is something I will always cherish.

Research is often arduous and quiet, but the Roman lab with Edilson Torres-González, Caleb Greenwell and Jeff Ritzenthaler truly broke that mold. Whether it was a discussion about experiments, an aluminum foil war, or sports, time in the lab was truly enjoyable. I would also like to thank Dr. Igor Zelko and Dr. Bert Watson for their continuous help. 
Additionally, I have to thank Dr. Cedric Francois, Jon Austin, Rachel Hanna and the rest of Apellis Pharmaceuticals and Revon Systems. Dr. Francois took a chance in offering me an internship, which has allowed me to work with the most amazing group of people. You've taught me skills that truly complemented what I was learning in the research lab.

Lastly, to Dr. Roman. Your enthusiasm about science and education is always contagious. No matter how busy your schedule, you always made time for me. I could not have asked for a better advisor with which to earn my degree.

"Friendship... is not something you learn in school. But if you haven't learned the meaning of friendship, you really haven't learned anything." - Muhammad Ali 


\begin{abstract}
THE ROLE OF NICOTINE, $\alpha 7$ NICOTINIC ACETYLCHOLINE RECEPTORS AND EXTRACELLULAR MATRIX REMODELING IN PULMONARY FIBROSIS
\end{abstract}

Glenn Ward Vicary

November $22^{\text {nd }}, 2016$

The median survival for idiopathic pulmonary fibrosis (IPF) patients from diagnosis is a dismal 3 years. This condition is characterized by pulmonary fibroproliferation and excess production and disordered deposition of extracellular matrix $(E C M)$ proteins resulting in obliteration of the original tissue architecture, loss of lung function and eventual death due to respiratory failure.

The main hindrance to the development of effective treatments against pulmonary fibrosis is the late detection of its progression and is often of unknown cause. Tobacco smoke represents the most important environmental factor linked to the development of pulmonary fibrosis, with over $60 \%$ of IPF patients current or ex-smokers, yet exactly how tobacco influences lung injury and repair is unknown. Research in this area has been hampered by the fact that tobacco is a very 
complex substance, containing thousands of chemicals. Due to this complexity, I have pursued a different approach and focused on factors, specifically nicotine, which might render the lung susceptible to fibrosis and contribute to the early pathophysiology of IPF.

In this dissertation, I extend the work of Dr. Jesse Roman's lab to investigate additional extracellular matrix modifications via nicotine exposure, including collagen type I. Investigating the cellular receptors and molecular mechanisms mediating the effects of nicotine on fibroblast collagen production/deposition and the potential role of nicotine-induced remodeling in rendering the host susceptible to pulmonary fibrosis are explored through 5 chapters: 1) The effects of nicotine on lung fibroblast proliferation and collagen expression/deposition in vitro and in vivo, and the cholinergic receptors responsible for these effects. 2) The effects of chronic nicotine exposure on injury-induced fibrosis. 3) The impact of chronic nicotine exposure on survival after bleomycin-lung injury. 4) A new diagnostic physiological formula for earlier detection of pulmonary fibrosis progression in IPF patients. 5) A clinical review on Hermansky-Pudlak syndrome, an orphan disease characterized by the natural formation of pulmonary fibrosis. This work provides a detailed understanding of the mechanisms by which tobacco promotes lung remodeling, leading to the development of better tools for diagnostic tracking, care and treatment of these patients. 


\section{TABLE OF CONTENTS}

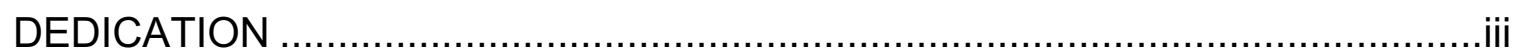

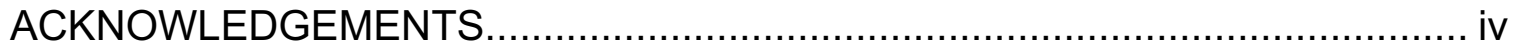

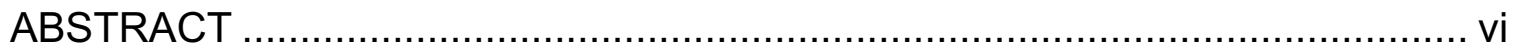

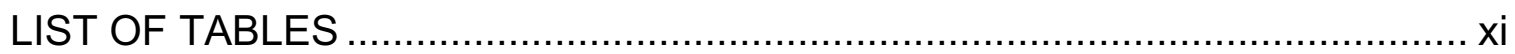

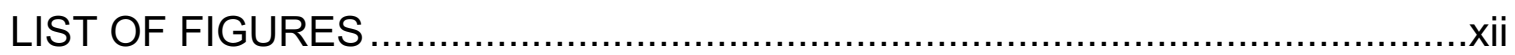

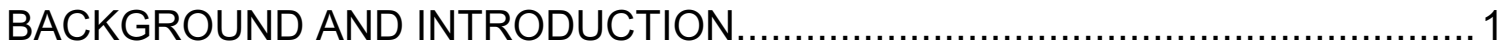

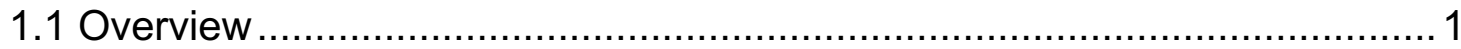

1.2 Prevalence of Idiopathic Pulmonary Fibrosis (IPF) .................................. 1

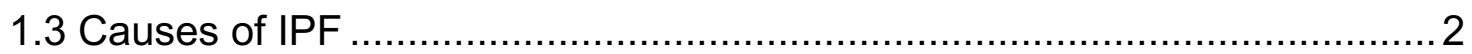

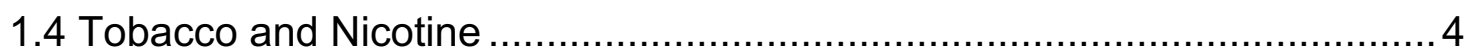

1.5 Mechanisms of Idiopathic Pulmonary Fibrosis ...................................... 5

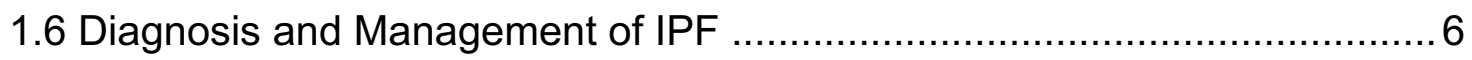

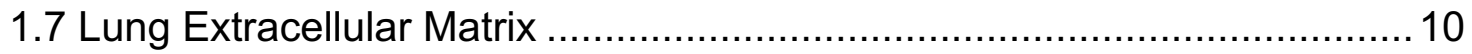

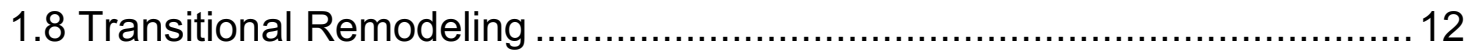

1.9 Nicotinic Acetylcholine Receptors (nAChRs) in Lungs ........................... 13

1.10 The Role of MEK-1/Erk pathway in mediating nAChR signaling .............16

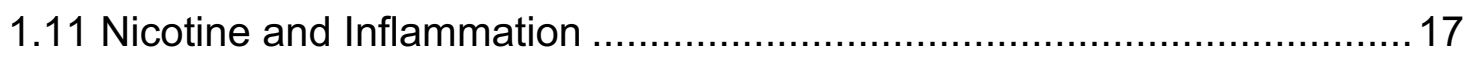

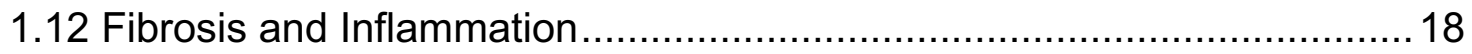

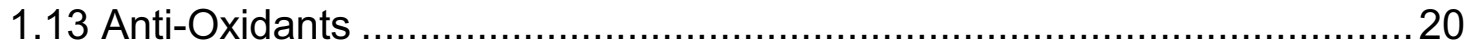

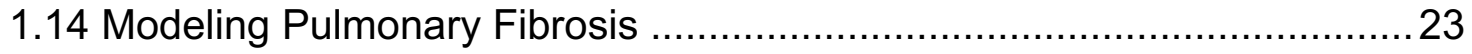

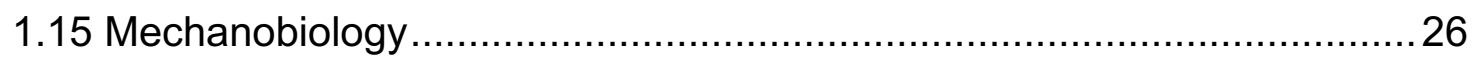

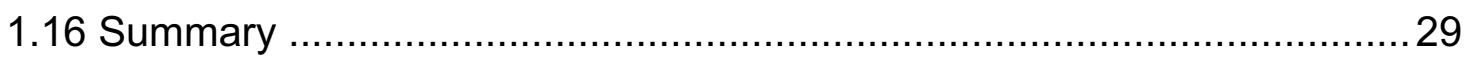

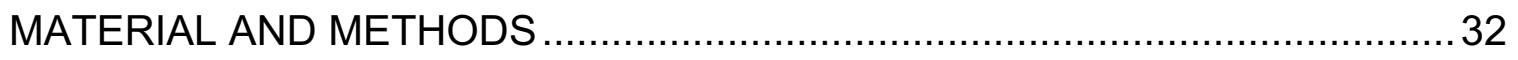

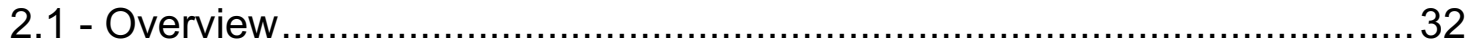

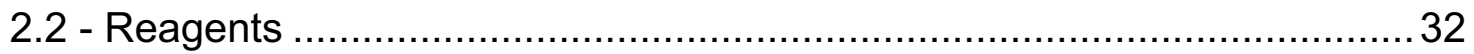

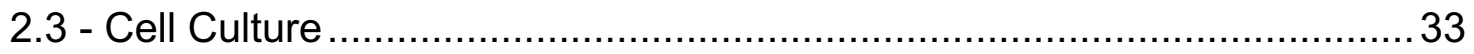

2.4 - Isolation and Detection of mRNAs by Reverse-Transcriptase Polymerase

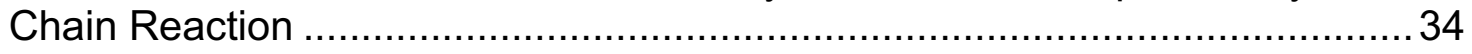

2.5 - Protein Detection via Western Blotting ............................................... 36 


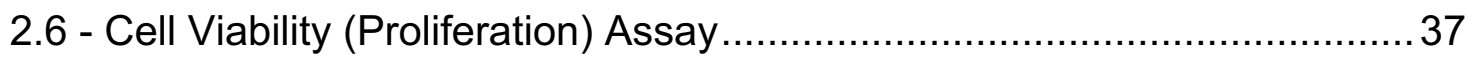

2.7 - Matrix Deposition and IL-1 $\beta$ Measurement ........................................... 38

2.8 - Superoxide Dismutase Activity Assay ................................................... 39

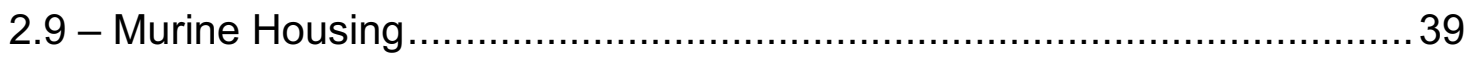

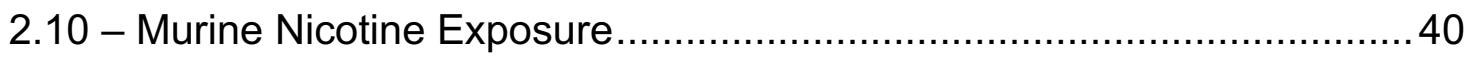

2.11 - Murine Cigarette Smoke Exposure …................................................. 40

2.12 - Murine Bleomycin-Induced Injury ……......................................... 41

2.13 - Murine Bronchoalveolar Lavage (BAL) ........................................ 41

2.14 - Murine Histological Analysis ........................................................ 41

2.15 - Protein Detection via Immunohistochemistry ................................... 42

2.16 - Murine Lung Decellularization .................................................... 43

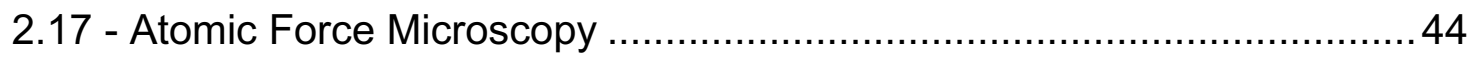

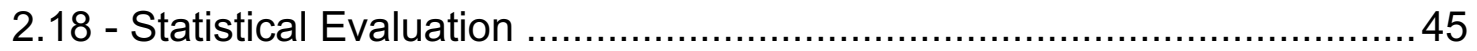

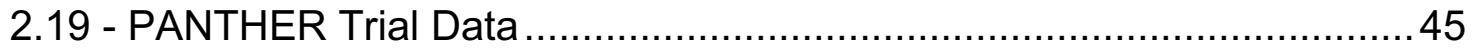

2.20 - PANTHER Trial Statistical Analysis .................................................. 46

2.21 - PANTHER Trial Human Subject Protection ........................................ 47

THE EFFECTS OF NICOTINE ON LUNG FIBROBLAST PROLIFERATION AND COLLAGEN EXPRESSION/DEPOSTION IN VITRO AND IN VIVO, AND THE CHOLINERGIC RECEPTORS RESPONSIBLE FOR THESE EFFECTS ...........48

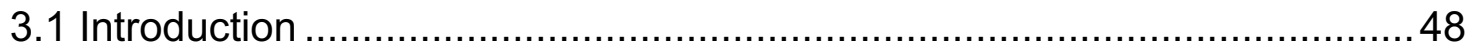

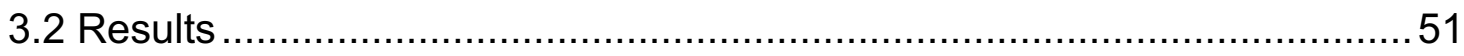

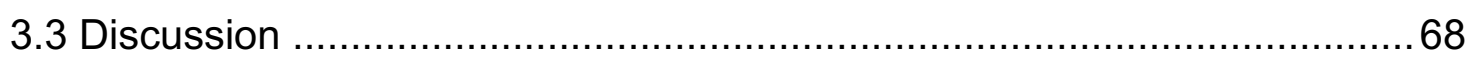

THE EFFECTS OF CHRONIC NICOTINE EXPOSURE ON INJURY-INDUCED

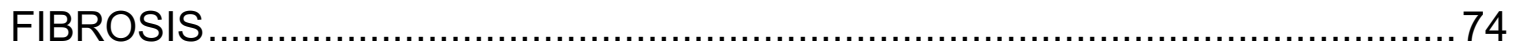

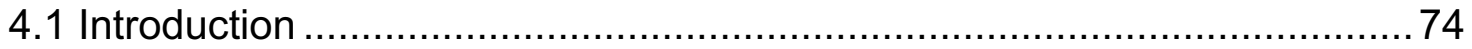

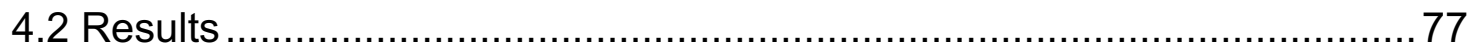

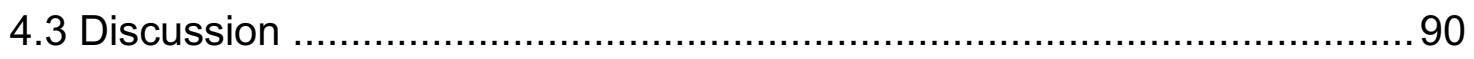

THE IMPACT OF CHRONIC NICOTINE EXPOSURE ON SURVIVAL AFTER

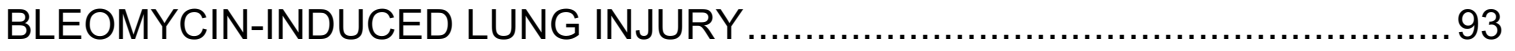

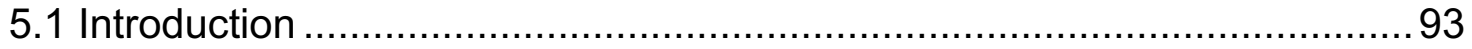

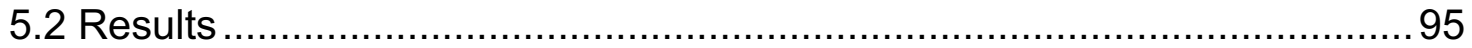

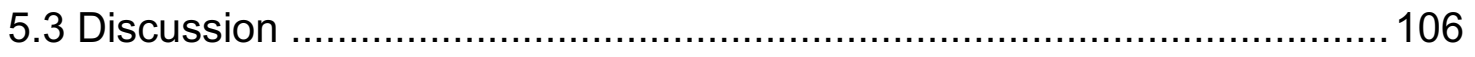




A NEW DIAGNOSTIC PHYSIOLOGICAL FORMULA FOR EARLIER
DECTECTION OF PULMONARY FIBROSIS PROGRESSION IN IPF PATIENTS
PULMONARY FIBROSIS IN HERMANSKY-PUDLAK SYNDROME




\section{LIST OF TABLES}

Table 1. Drop in 10\% FVC Patient Cohort Baseline Characteristics ................115

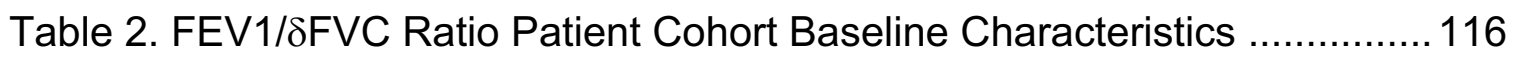

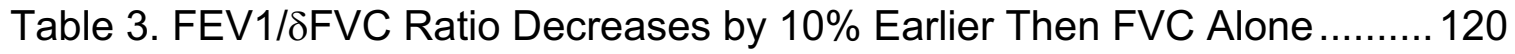

Table 4. Drop in 10\% FVC as Predictor of Hospitalization/Death .................... 122

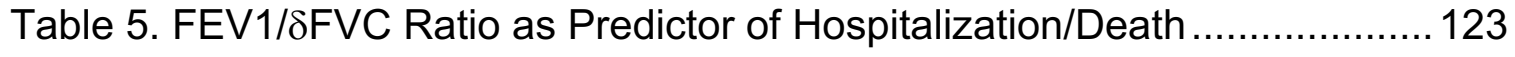

Table 6. Human Hermansky-Pudlak Syndrome (HPS) Variants ...................... 131

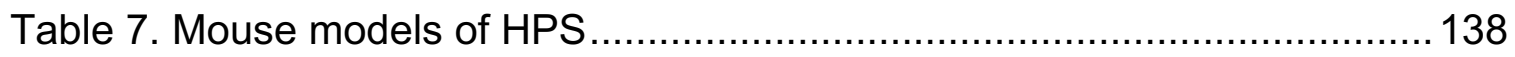

Table 8. Phenotype and cellular abnormalities in mouse models of HPS .........139 


\section{LIST OF FIGURES}

Figure 1. Classifying Interstitial Lung Diseases............................................. 3

Figure 2. Proposed Events of Idiopathic Pulmonary Fibrosis ............................ 8

Figure 3. Fibroblastic Foci in Idiopathic Pulmonary Fibrosis .............................. 9

Figure 4. Transitional Remodeling of the Lung Extracellular Matrix (ECM) ........14

Figure 5. Structure of Nicotinic Acetylcholine Receptors …............................ 15

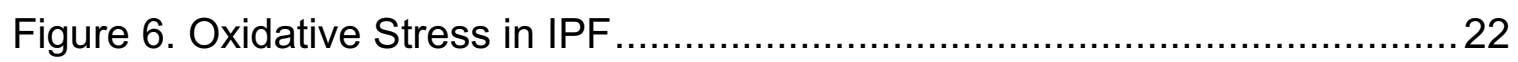

Figure 7. Bleomycin-Induced Model of Pulmonary Fibrosis ..............................25

Figure 8. Mechanisms of Atomic Force Microscopy .........................................30

Figure 9. Lung Mechanobiology ……............................................................ 31

Figure 10. Nicotine Stimulates Collagen Type I mRNA and Protein Expression 52 Figure 11. Nicotine Stimulates Collagen Type I mRNA and Protein Expression at

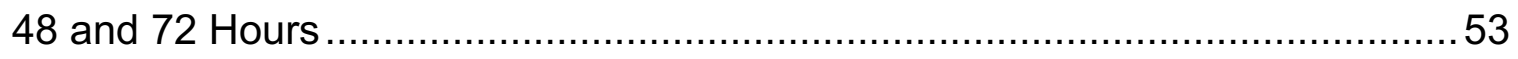

Figure 12. Nicotine Induces Collagen Type I Through $\alpha 7$ nAChRs ..................54

Figure 13. Nicotine Stimulates the Proliferation of Lung Fibroblasts via $\alpha 7$

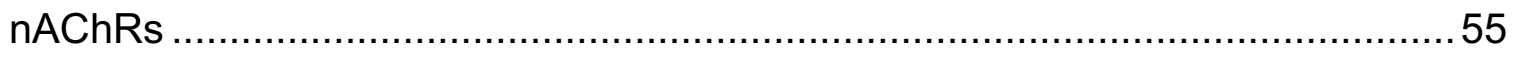

Figure 14. Nicotine Stimulates the Proliferation of Lung Fibroblasts via Induction

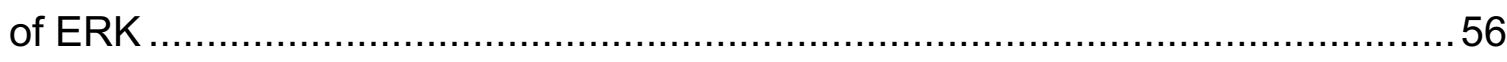

Figure 15. Nicotine Stimulates Collagen Type I mRNA Expression in Lung

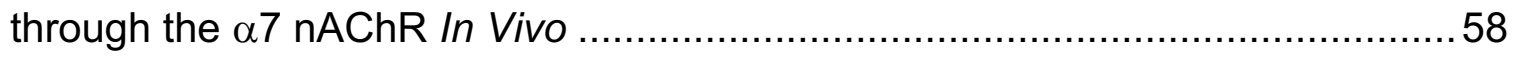


Figure 16. Nicotine Stimulates p-Erk, p-Smad3 and Collagen Type I Protein Expression in Lung through the $\alpha 7$ nAChR In Vivo..........................................59

Figure 17. Nicotine Stimulates Collagen Expression in Lung In Vivo..................60 Figure 18. Nicotine-Induced Collagen Expression Mediated via $\alpha 7$ nAChRs in Lung In Vivo 61

Figure 19. Matrices Derived from Nicotine-Treated Fibroblasts Stimulate IL-1 $\beta$ Expression in Monocytic Cells Through the $\alpha 2 \beta 1$ Integrin Receptor 63

Figure 20. Pro-Inflammatory Matrices Derived from Nicotine-Treated Fibroblasts Mediated by $\alpha 7 \mathrm{nAChR}$

Figure 21. Pro-Inflammatory Matrices Derived from Nicotine-Treated Fibroblasts Blocked by $\alpha 7$ nAChR Inhibitor 65

Figure 22. Pro-Inflammatory Matrices Derived from Nicotine-Treated Fibroblasts Blocked by MEK-1 Inhibitor 66

Figure 23. Nicotine Stimulates IL-1 $\beta$ Expression in Lung In Vivo 67

Figure 24. Nicotine Stimulates Fibronectin Protein Expression Through $\alpha 7$ nAChRs 78

Figure 25. Nicotine Stimulates $\alpha$-Smooth Muscle Actin ( $\alpha$-SMA) mRNA Expression

Figure 26. Nicotine Stimulates mRNA Fibronectin Expression through $\alpha 7$ nAChRs In Vivo 80 Figure 27. Nicotine Stimulates $\alpha$-SMA Protein Expression through $\alpha 7$ nAChRs In Vivo 81 
Figure 28. Nicotine Stimulates Fibronectin Expression through $\alpha 7$ nAChRs In Vivo

Figure 29. Bronchoalveolar Lavage (BAL) Fluid from Nicotine-Treated Mice Induced Fibronectin Expression in NIH3T3 Fibroblasts 83

Figure 30. Nicotine Induces Bleomycin-Induced Fibrosis ...............................86

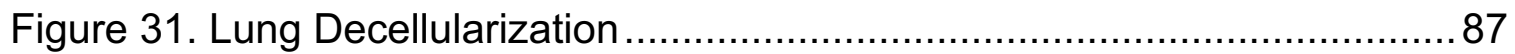

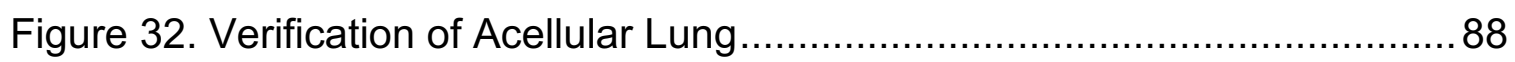

Figure 33. Nicotine Did Not Increased Stiffness of Bleomycin-Treated Lungs....89 Figure 34. Nicotine Induces Protection Against Dead Caused by BleomycinInduced Lung Injury 98

Figure 35. Nicotine-Treatment Does Not Affect Bleomycin-Induced Weight Loss

Figure 36. Nicotine-Induced Protection (107 Days) Against Bleomycin-Induced

Death Showed No Differences in Fibrosis. 100

Figure 37. Nicotine-Induced Protection (207 Days) Against Bleomycin-Induced

Death Showed No Differences in Fibrosis..... 101

Figure 38. Nicotine-Induced Protection Against Bleomycin-Induced Death Not Connected to Inflammation 102

Figure 39. Nicotine-Induces Superoxide Dismutase 1 (SOD1) mRNA Expression 103

Figure 40. Nicotine-Induces SOD1 mRNA Expression and Activity In Vivo ...... 104

Figure 41. Nicotine-Induces SOD1 Activity In Vivo ....................................... 105

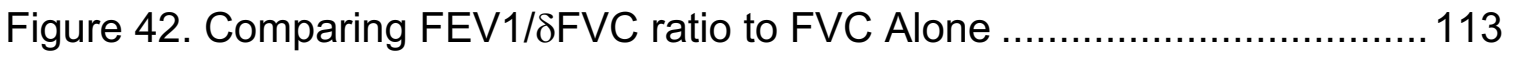


Figure 43. PANTHER Trial Enrollment and Outcomes

Figure 44. Distribution of FEV1, FVC, FEV1/FVC and FEV1/8FVC Values ......119

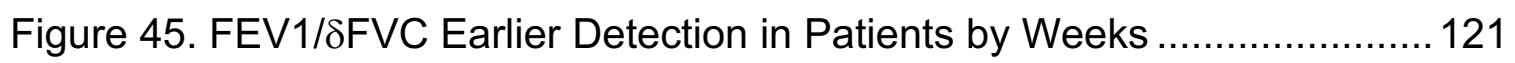

Figure 46. Death and Hospitalization Receiver Operating Characteristics (ROC)

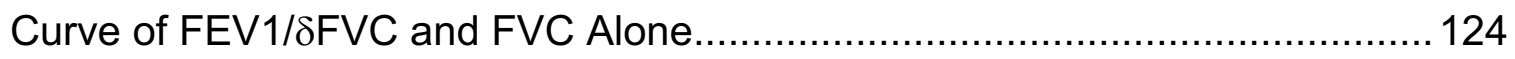

Figure 47. Patient Hospitalization/Death by Weeks ..................................... 125

Figure 48. Lysosomal-Related Organelles (LROs) in HPS ............................ 133

Figure 49. Examples of Chest CT findings in Subjects with HPS-PF ............... 144

Figure 50. Dissertation Summary ........................................................ 158

Figure 51. Cigarette Smoke Induces Fibronectin In Vivo …........................... 166

Figure 52. Cigarette Smoke Induces Fibronectin Time-Dependently In Vivo.... 167

Figure 53. Bleomycin Treatment is Associated with Increased Metastases in

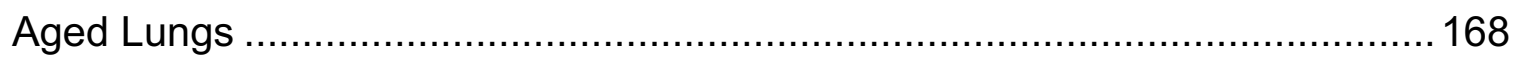




\section{CHAPTER I}

\section{BACKGROUND AND INTRODUCTION}

\subsection{Overview}

The lung is a dynamic organ, constantly expanding and contracting while being exposed to environmental and chemical challenges. To handle these insults, the lung readily repairs itself against a range of antigens and environmental pathogens. Correct lung repair allows for normal lung function, but when disrepair occurs, a variety of diseases can occur, including idiopathic pulmonary fibrosis. Lung remodeling caused by disrepair is often drastic, affecting protein composition, lung structure, elasticity, and oxygen diffusion, making it progressively difficult for patients to do even basic activities such as walking and talking.

\subsection{Prevalence of Idiopathic Pulmonary Fibrosis (IPF)}

Idiopathic pulmonary fibrosis (IPF) is a chronic disease that affects over 100,000 Americans [1]. The median survival for patients from diagnosis is a dismal 
3 years [2]. IPF is usually diagnosed in older patients, with more than $85 \%$ of patients over 50 years old and an increased prevalence in males [2-9]. Surprisingly, the number of IPF cases appears to be increasing; likely due to the aging population, increased patient and physician awareness of IPF and improved computerized tomography (CT) imaging [10].

IPF is classified as an interstitial lung disease (ILD), which is an umbrella term for a large group of pulmonary disorders characterized by progressive remodeling of pulmonary tissue. ILDs are characterized by known and unknown causes, consisting of over 200 diseases including sarcoidosis and idiopathic interstitial pneumonias (IIPs). IIPs are subclassified based on histological appearance, with the most common and severe form of IIP being IPF; which accounts for $20-30 \%$ of ILDs (Figure 1) [11, 12]. IPF is diagnosed by exclusion of other diseases when there is evidence of impairment of lung function and presence of a histopathological pattern of usual interstitial pneumonia (UIP).

\subsection{Causes of IPF}

Environmental factors (e.g., allergens, tobacco), occupational exposures (e.g. asbestos and silica exposure), genetic factors (e.g. surfactant-related proteins, telomerase, HPS) and viral factors (herpesvirus) have been associated with the development of pulmonary fibrosis, but its etiology can be elusive [1, 1317]. 


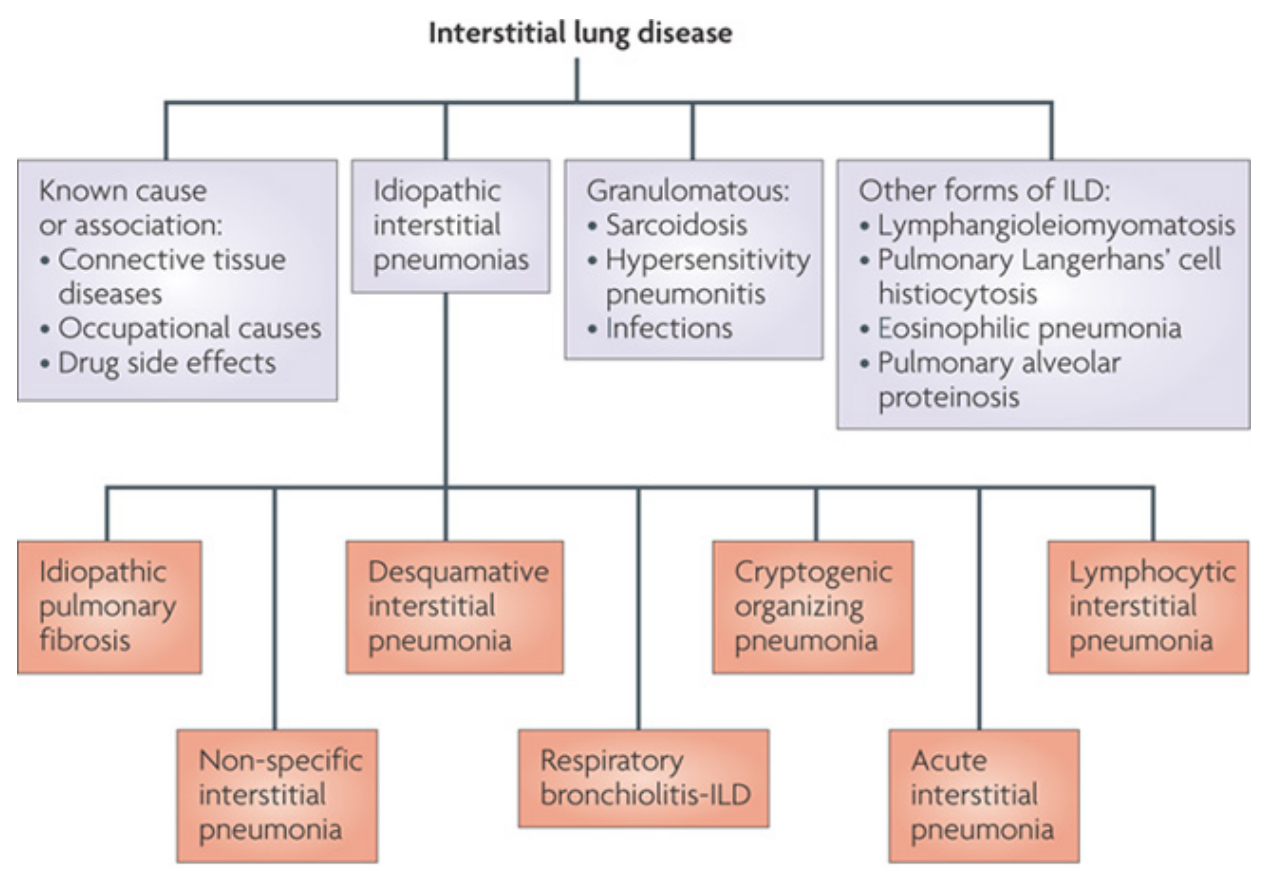

Nature Reviews | Drug Discovery

\section{Figure 1. Classifying Interstitial Lung Diseases}

Reprinted with permission from Nature Publishing Group. [Nat Rev Drug Discover] (du Bois, R.M., Strategies for treating idiopathic pulmonary fibrosis, 2010. 9, p. 124-140) Copyright 2010

License \#: 3972681241289

Idiopathic pulmonary fibrosis is classified as an interstitial lung disease, which is an umbrella term for a large group of pulmonary disorders characterized by progressive remodeling of pulmonary tissue. Interstitial lung diseases can be subdivided into four broad categories based on histological appearance. Idiopathic pulmonary fibrosis is the most lethal ILD and its diagnosis depends on the exclusion of other diseases. 
Underlying amplifying factors like aging and epigenetic changes are thought to influence the progressive nature of pulmonary fibrosis [18]. Most cases of IPF are sporadic with $4 \%$ of patients being familial [19-22]. The familial form of IPF tends to present at a younger age when compared with the more common idiopathic form.

\subsection{Tobacco and Nicotine}

Of all the environmental risk factors, tobacco smoke represents a major factor linked to the development of IPF, with over $60 \%$ of IPF patients being current or ex-smokers [1, 23-25]. However, studying the role of tobacco in IPF has been hampered by the complex nature of tobacco smoke, which contains over 7,000 chemicals $[26,27]$. Due to the complexity of tobacco, attention has recently turned to the potential role of nicotine in mediating the effects of tobacco smoke.

Nicotine is an alkaloid present in tobacco and represents $\sim 0.6-3.0 \%$ of the dry weight of tobacco and is known for driving the addictiveness of cigarettes. Nicotine's unique properties allow $30 \%$ of it to be instantly absorbed and reach the brain within 7 seconds $[28,29]$. Studies have shown smokers to have average nicotine levels of $10-50 \mathrm{ng} / \mathrm{ml}(0.3 \mu \mathrm{M})$ in blood plasma [30]. The metabolism of nicotine varies between species, with a plasma half-life of 2 hours in humans and approximately 6-7 minutes in mice [30]. In humans, the metabolism rate of nicotine is affected by age, race, and gender. Women and younger smokers have been 
reported to metabolize nicotine quicker [31, 32]. Additionally, caucasians cleared nicotine more rapidly than Asians and African Americans [30].

Nicotine not only drives addiction, but affects several biological processes, including cell proliferation, apoptosis, migration, invasion, angiogenesis, inflammation and immunity [33]. Additional studies have unveiled that nicotine also affects lung development through increased branching and increasing nAChR expression [34, 35]. Thus, it appears that nicotine is not just responsible for tobacco dependence, but might also contribute to the pathogenesis of tobaccorelated disorders, including IPF.

\subsection{Mechanisms of Idiopathic Pulmonary Fibrosis}

The exact mechanisms which contribute to the development of IPF are unknown. However, current models of pulmonary fibrosis pathogenesis indicate epithelial cell apoptosis and dysfunction, likely type I alveolar epithelial cells (AECs), as the inciting cause of IPF [36]. Type I AECs are believed to control pulmonary structural mesenchymal cells through the secretion of various mediators and cell-cell contact [37]. When injured, type I AECs are thought to signal type II AECs to undergo hyperplastic proliferation to cover the exposed basement membranes. After normal repair, the hyperplastic type II AECs will undergo regulated apoptosis and any remaining cells will differentiate to become type I AEC [38-40]. In contrast, disrepair leads to scar tissue formation, which is usually self-limited, but can become progressive, with ongoing lung parenchymal 
destruction and replacement by pathologic fibrosis with the activation of fibroblasts (Figure 2).

Under these conditions of disrepair, Transforming Growth Factor- $\beta$ (TGF$\beta$ ) stimulation may activate fibroblasts to differentiate into $\alpha$-smooth muscle actin ( $\alpha$-SMA) expressing myofibroblasts [18, 36, 41]. The origins of these fibroblasts (myofibroblasts) are unclear, but combinations of mechanisms are hypothesized, including the transdifferentiation of epithelial cells (epithelial-mesenchymal transformation (EMT)), the differentiation of fibrocytes and stem cells, and the proliferation of resident fibroblasts [42-44].

Regardless of the source of origin, the interplay between dysfunctional epithelial cells and activated myofibroblasts seems to drive unrestrained collagen deposition and tissue scarring 'fibrotic foci', resulting in obliteration of the original architecture, loss of lung function and, eventual death due to respiratory failure (Figure 3) [45-48]. In IPF patients, areas of AEC apoptosis and foci of $\alpha$-SMApositive myofibroblasts were detected together, consistent with the mechanism proposed above [49].

\subsection{Diagnosis and Management of IPF}

There is no cure for IPF, so treatment focuses on slowing the progression of fibrosis and improving patient quality of life. Avoidance of cigarette smoke and other pulmonary irritants is critical with daily exercise recommended to avoid deconditioning. Recently, the FDA has approved two drugs for IPF treatment, 
Pirfenidone and Nintedanib [50-53]. It should be emphasized that these drugs are not curative, but instead appear to slow down progression in IPF. Also, the effectiveness of these drugs in other conditions resulting in pulmonary fibrosis has not been studied, thus, current interventions for pulmonary fibrosis remain suboptimal. Pirfenidone is a small molecular TGF- $\beta$ inhibitor, whereas Nintedanib is a tyrosine kinase inhibitor that targets various growth factor receptors including platelet derived growth factor (PDGF), fibroblast growth factor (FGFR) and vascular endothelial growth factor (VEGF) receptors [50, 52, 54]. These drugs also have undesirable side effects, so supportive therapy is an additional approach to management of IPF. Additional therapies include supplemental oxygen for hypoxemia, pulmonary rehabilitation and lung transplantation in appropriate candidates.

The main hindrance to the development of effective treatments against pulmonary fibrosis is due to late diagnosis. For this reason, efforts should be directed at novel therapeutics for prevention of lung remodeling or early detection through the identification of specific early biomarkers of disease. It is for this reason that I have pursued a different approach and focused on the factors that render the lung susceptible to fibrosis and contribute to the early pathophysiology of IPF, which might help gain insight into the overall mechanisms that lead to the development of this disease and the factors that regulate its progression. 


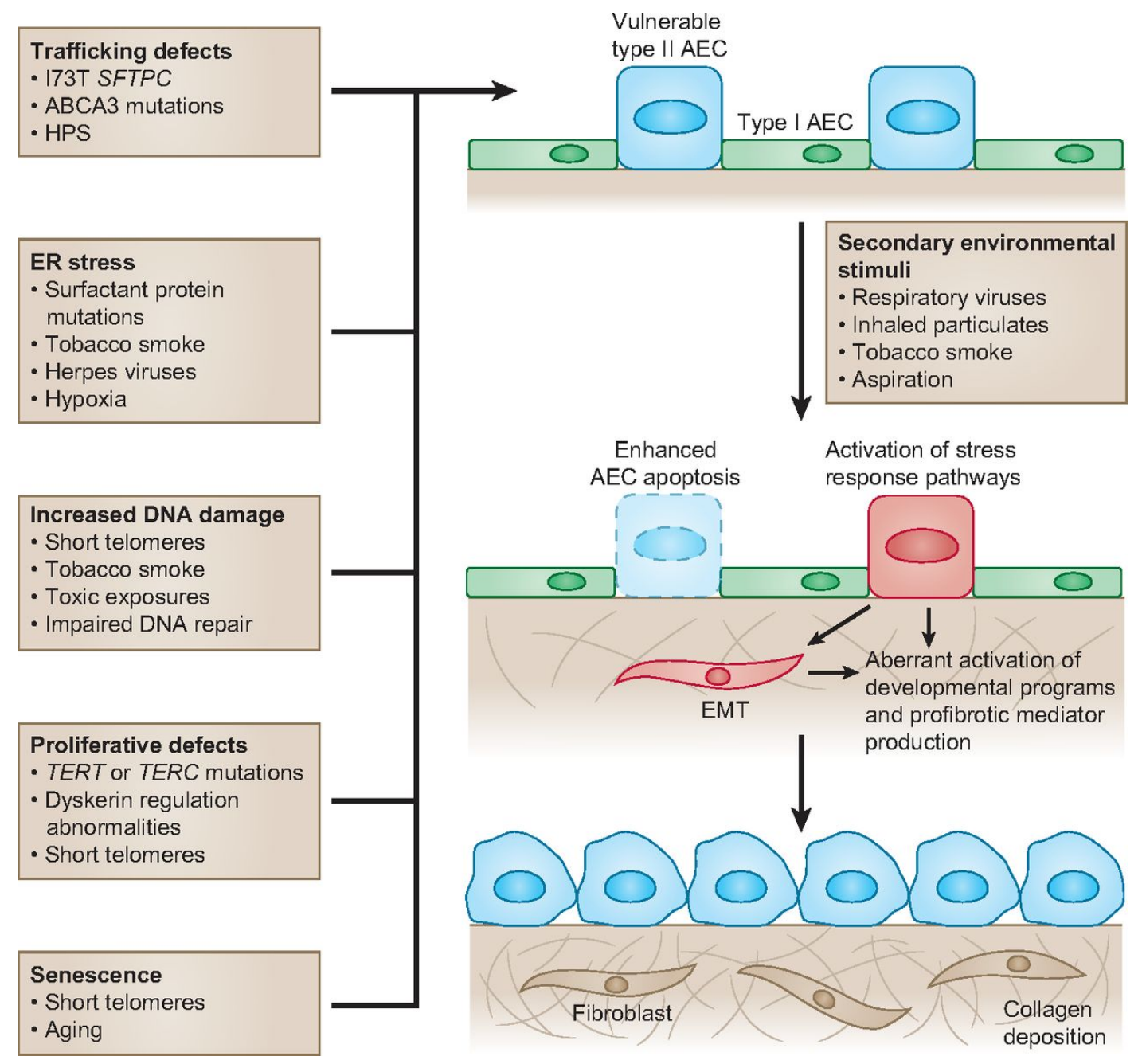

Figure 2. Proposed Events of Idiopathic Pulmonary Fibrosis

Reprinted with permission from The Company of Biologists Ltd. [Dis. Model. Mech.] (Kropski J. A. et Al., Genetic studies provide clues on the pathogenesis of idiopathic pulmonary fibrosis, 2013. 6(1): p. 9-17) Copyright 2012

Reproduction Lic\# https://creativecommons.org/licenses/by-nc-sa/3.0

Various genetic factors and environmental exposures produce a 'vulnerable' alveolar epithelial cell (AEC) population. Exposure to a secondary recurrent or persistent injury leads to enhanced AEC apoptosis, as well as aberrant activation of stress response pathways. Ongoing type II AEC dysfunction leads to scar formation and progressive loss of lung architectural complexity. This culminates into progressive fibrotic pulmonary tissue remodeling. 

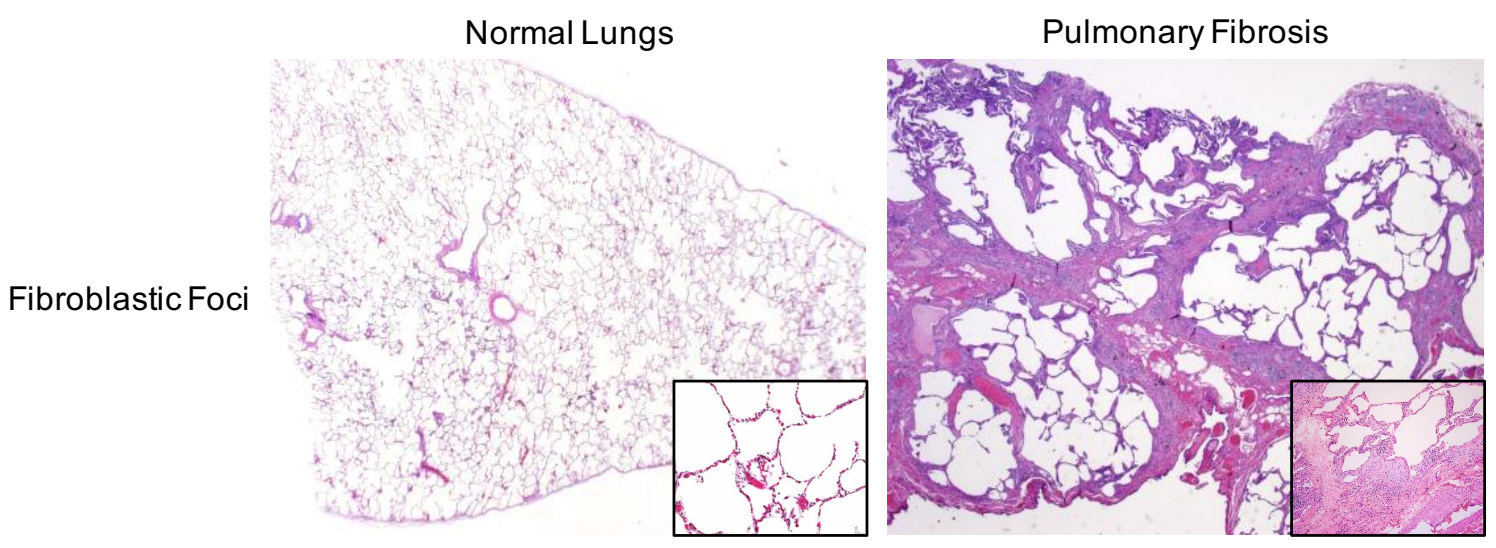

Figure 3. Fibroblastic Foci in Idiopathic Pulmonary Fibrosis

Copyright Permissions not required for this type of use. (Lederer, D., A new era in IPF: Diagnosing Idiopathic Pulmonary Fibrosis) http://www.pilotforipf.org/ipf-resources/slide-library

Idiopathic pulmonary fibrosis is characterized by unrestrained collagen deposition and tissue scarring from fibroblasts, forming 'fibrotic foci', resulting in obliteration of the original extracellular matrix as seen here in histological staining by hematoxylin and eosin staining. 


\subsection{Lung Extracellular Matrix}

Extracellular matrix remodeling is the main characteristic of pulmonary fibrosis. The extracellular matrix (ECM) was historically believed to be a noncellular scaffolding of fibrous proteins, glycoproteins and proteoglycans for cells to adhere, but recent research has shown that the ECM plays many crucial roles in homeostasis and disease [55]. Now, the ECM is known to affect important biochemical and biomechanical signaling between cells, including cellular differentiation [56].

The pulmonary ECM consists of basement membranes and the interstitial spaces. These basement membranes are found under epithelial and endothelial cells which form the interstitial spaces of the parenchyma [57]. Within this interstitium is where fibroblasts, which are mainly responsible for ECM production, reside. The ECM of murine and human lungs have been characterized, showing the majority of the matrisome is conserved $[58,59]$. Under homeostatic conditions, the ECM is tightly regulated for normal development and wound healing. Alterations in pulmonary ECM are suspected to play a role in disorders affecting all lung compartments (airways, vessels, alveolar units), including IPF, Chronic Obstructive Pulmonar Disease (COPD) and sarcoidosis.

Fibronectin is an ECM glycoprotein highly expressed in injured tissues, and is considered a sensitive marker of tissue injury and activation of tissue remodeling [60-62]. In the injured lung, fibronectin is deposited over denuded basement membranes where it is thought to support the migration of alveolar epithelial cells 
during repair [61]. It promotes the proliferation of fibroblasts, aids in the deposition of collagen, stimulates the chemotaxis of monocytes and other immune cells and induces cytokine production in macrophages [63-69]. Excessive fibronectin deposition, however, has been hypothesized to promote disrepair [70]. Fibronectin expression and fibroproliferation were increased in the lungs of smokers [71-74]. Also, clinical studies have shown increased fibronectin content within the bronchoalveolar lavage fluid of smokers [75].

Fibrillar collagen is another matrix component highly expressed in lung tissues during injury and repair, and its expression signals activation of more permanent tissue remodeling. Collagen type $\mathrm{I}$ is the most common form of collagen in the human body and is highly abundant within connective tissues, including tendon, ligament, skin, and lung tissue. Each rope-like procollagen molecule is made up of three chains: two pro- $\alpha 1$ (I) chains, which are produced from the COL1A1 gene, and one pro- $\alpha 2$ (I) chain, which is produced from the COL1A2 gene. After processing, the resulting mature collagen molecules arrange themselves into long, thin fibrils. Individual collagen molecules are then crosslinked to one another within these fibrils, thereby forming strong collagen fibrils [76].

These ECM proteins, together with proteoglycans and other matrix components compose the acellular structure of the lung. The controlled deposition and turnover of these ECM proteins is essential for maintaining tissue integrity. Alterations in these processes lead to aberrant tissue remodeling and fibrotic lung diseases. 


\subsection{Transitional Remodeling}

The connection between nicotine and tissue remodeling seen in pulmonary fibrosis has yet to be completely elucidated. To date, there are no data suggesting nicotine, alone, can promote pulmonary fibrosis. The Roman lab has previously shown that nicotine exposure induces fibronectin deposition by pulmonary fibroblasts. Additionally, increased fibronectin deposition was detected in the lungs of animals chronically exposed to nicotine, but without overt alterations in overall lung architecture [77].

Instead, this dissertation provides evidence of a process termed transitional remodeling, which might precede the dramatic tissue alterations and potentiate pulmonary fibrosis progression. Transitional remodeling is defined as subtle alterations in the ECM composition deposition and assembly, including fibronectin and collagen, without causing overt change in tissue architecture. Nicotine exposure induces transitional remodeling, but this could be reversible and, in fact, in most injuries, the body may undergo transitional remodeling as part of the normal wound healing process. As such, the body returns to normal. However, the implications of transitional remodeling in disrepair are not fully understood. If the lung experiences a second hit (e.g. bleomycin), transitional remodeling may render the host susceptible to disrepair through the pro-inflammatory effects of matrix fragments and by its ability to promote the deposition of other more permanent ECM proteins (Figure 4) [78]. 


\subsection{Nicotinic Acetylcholine Receptors (nAChRs) in Lungs}

As presented above, nicotine promotes transitional remodeling. However, the mechanisms involved in this process are unclear. Nicotine interacts with the body through receptors capable of signal transduction termed nicotinic acetylcholine receptors (nAChRs) (Figure 5) [79, 80]. nAChRs are a family of multimeric acetylcholine-triggered cation channel proteins that form the excitatory neurotransmitter receptors on muscles and nerves in the peripheral nervous system. In addition to the nervous system, nAChRs have been found in lymphocytes [81], leukocytes [82], monocytes [83], keratinocytes [84], and NIH3T3 fibroblasts [85]. These receptors are pentameters made up in homomeric and heteromeric fashion. The homomeric $(\alpha 7)_{5}$ is the most common nAChR, containing five $\alpha 7$ subunits and is the most abundant homomeric form in the body. Binding of a ligand (acetylcholine/nicotine) leads to depolarization of the membrane and generation of an action potential that spreads along the surface of cell membranes.

Evidence of $\alpha 3, \alpha 5, \alpha 7$ nAChR subunits has been detected in both human and mouse bronchial epithelial cells [86]. $\alpha 7 \mathrm{nAChRs}$ have been detected in airway epithelial cells of developing primate lungs around large airways and blood vessels, free alveolar macrophages, alveolar type II cells and pulmonary neuroendorcrine cells [34]. 


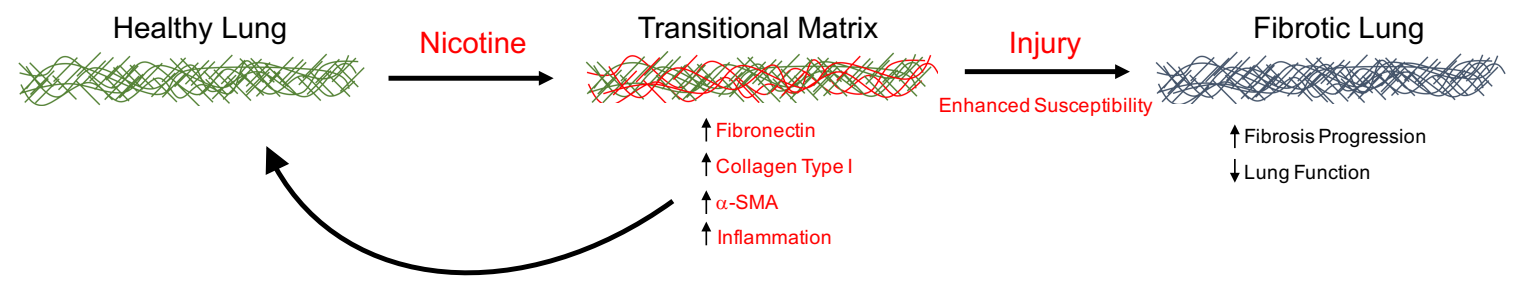

Injury Resolution

Figure 4. Transitional Remodeling of the Lung Extracellular Matrix (ECM)

Copyright Permissions not required for this type of use. (Adapted from Poole, L. and Arteel, G., Transitional Remodeling of the Hepatic Extracellular Matrix in Alcohol-induced Liver Injury) https://www.hindawi.com/journals/bmri/aip/3162670/

Nicotine exposure induces ECM proteins and subclinical remodeling forming a transitional extracellular matrix. This transitional matrix, while it causes no detrimental changes in the lung, may contribute to disrepair and inflammation after injury. If the insult is removed, the transitional matrix may resolve back to a normal state. Exposure to a secondary injury may progress to a fibrotic matrix via increased ECM synthesis and blunted ECM degradation, ultimately leading to pulmonary fibrosis. 

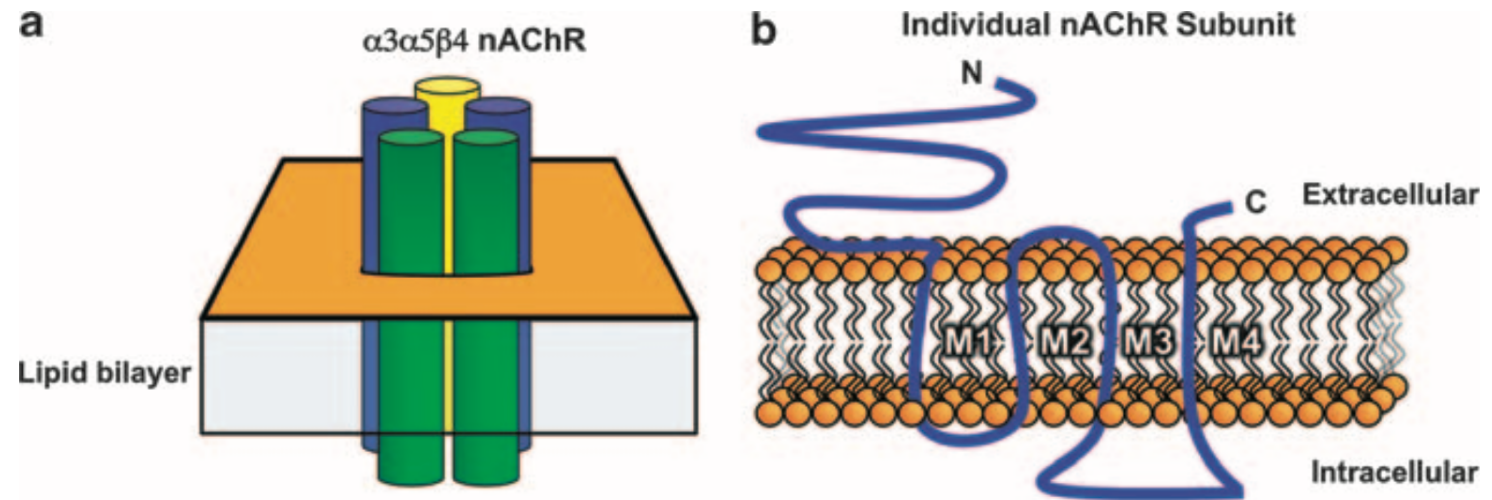

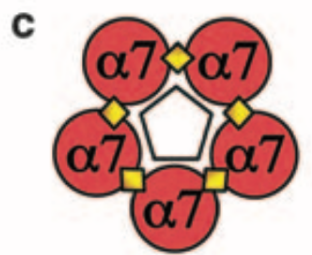

Homomeric nAChR

(e.g. $\alpha 7)$

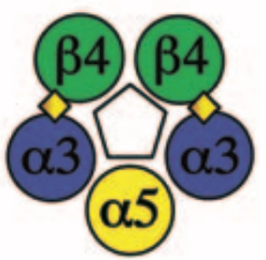

Heteromeric nAChRs (e.g. $\alpha 3 \beta 4 \alpha 5$ and $\alpha 3 \beta 4$ )

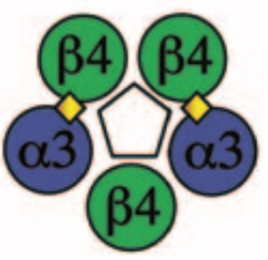

$\beta 4$

Figure 5. Structure of Nicotinic Acetylcholine Receptors

Reprinted with permission from Nature Publishing Group. [Oncogene] (Improgo, M.R., From smoking to lung cancer: the CHRNA5/A3/B4, 2010. 29, p. 4874-4884) Copyright 2010

Nicotinic Acetylcholine Receptors (nAChRs) are a family of multimeric acetylcholine-triggered cation channel proteins that form the excitatory neurotransmitter receptors on muscles and nerves in the peripheral nervous system. (A) Schematic representation illustrating the pentameric arrangement of subunits in an assembled nAChR. (B) Conserved domains of a $n A C h R$ subunit including the amino $(\mathrm{N})$ and carboxy $(\mathrm{C})$ terminals, transmembrane segments (M1-M4) and the intracellular loop. (C) Assembly of heteromeric and homomeric nAChR subtypes. Individual nAChR subunits are represented as colored circles, with diamonds representing ligand-binding sites. Pentagons in the center of each pentamer represent the pore region, which is selective for cations (e.g. Calcium). 
The role of nAChRs in the lung is largely unknown. Acetylcholine is produced by several non-neuronal lung cells as a regulator of bronchomotor tone and mucus secretion from airway submucosal glands in lungs. Nicotine-nAChR interactions can affect lung development and ECM deposition. Dr. Roman's lab has demonstrated that nicotine induces fibronectin expression in lung fibroblasts via $\alpha 7 \mathrm{nAChRs}$ [77]. This increase in matrix proteins suggests that nicotine might induce myofibroblastic features in lung fibroblasts, which has been associated with pulmonary fibrosis [87]. Dr. Elliot Spindel's group has published articles showing $\alpha 7 \mathrm{nAChR}$ expression was increased in animals exposed to nicotine, correlating with increased tissue remodeling (collagen deposition) in surrounding large airways and vessels [77, 88-94]. Nicotine- $\alpha 7 \mathrm{nAChR}$ interactions also influence lung branching morphogenesis and result in airway thickening and dysfunction [34, $35,91]$.

\subsection{The Role of MEK-1/Erk pathway in mediating nAChR signaling}

The intracellular pathways by which nicotine-nAChR interactions promote lung tissue remodeling are not fully characterized. Fibroblasts from IPF patients show sustained mitogen-activated protein kinase (MAPK) induction and activation, a downstream target of the MEK-1/Erk pathway [95, 96]. Fibrotic agents, like crystalline silica and asbestos, promote activation of MEK-1, which increases the level of pro-inflammatory cytokines [97]. MEK-1/Erk also induces tumor necrosis factor- $\alpha$ (TNF- $\alpha)$ through the pro-fibrotic growth factor TGF- $\beta$ [98]. 
As a proof of concept for clinical targeting, a MEK inhibitor prevented TNF$\alpha$ induction and even reversed existing TGF- $\beta$-induced lung fibrosis [99]. Additionally, a different MAPK/Erk inhibitor prevented bleomycin-induced fibrosis [100]. No studies have been published on the effects of MEK-1 inhibition in humans. MEK-1/Erk could provide a future clinical target for preventing nicotine induced lung changes.

\subsection{Nicotine and Inflammation}

In addition to ECM, the regulation of the immune system is a critical physiological process during pulmonary development, healing, trauma, or infection. Controlled production of cytokines, such as interleukins (ILs) and TNF- $\alpha$ induce beneficial inflammatory responses that promote local coagulation to confine infection and tissue damage [101]. However, unrestricted production of these cytokines can cause more damage than the original injury.

Acetylcholine is an important neurotransmitter that controls the production of pro-inflammatory cytokines. Acetylcholine (endogenous) and nicotine (exogenous) can either bind to muscarinic receptors, which are G-protein-coupled receptors, or nAChRs, which are ligand-gated ion channels [102].

How nicotine affects inflammation is still being elucidated. nAChRs are believed to induce different inflammatory responses based on receptor affinities in multiple immune cell types [103-107]. $\alpha 7$ nAChR ligands (acetylcholine and nicotine) are normally considered anti-inflammatory due to activation of the 
cholinergic anti-inflammatory pathway. Nicotine has been shown to reduce proinflammatory cytokine expression in monocytes [108]. Interestingly, nicotine is more specific than acetylcholine at inhibiting the production of pro-inflammatory cytokines from macrophages and monocytes and this appears to be $\alpha 7 \mathrm{nAChR}$ specific [101, 109-111]. Additionally, an $\alpha 7$ agonist PNU-282987 was used in a murine model of LPS-induced acute lung injury reduced neutrophil recruitment and IL- $1 \beta$, TNF- $\alpha$, IL- 6 and IL-10 cytokine levels in the bronchoalveolar lavage fluid. In addition, the frequency of M1 macrophages suggests a change in the macrophage profile [112]. Nicotine can also affect immune cell function, inhibiting phagocytic activity in neutrophils while enhancing neutrophil elastase release without affecting superoxide production [113, 114]. Additionally, nicotine induces neutrophil traps, which can contribute to tissue damage and inflammation [115]. Thus, nicotine- $\alpha 7 \mathrm{nAChR}$ interactions appears to directly decrease inflammation in certain cells, but systemic exposure and indirection activation still need to be investigated.

\subsection{Fibrosis and Inflammation}

Pulmonary fibrosis has traditionally been considered related to extensive ECM remodeling with subdued inflammation, but more recent research connects innate and adaptive immune responses to fibrogenesis. TGF- $\beta 1$ is considered a central mediator in pulmonary fibrosis and is the most studied soluble mediator in addition to playing a central role in promoting EMT [116, 117]. Additionally, 
activated fibroblasts are highly responsive to additional growth factors/proinflammatory cytokines (IL-1 $\beta$, IL-6, IL-13, IL-33, TNF- $\alpha$, and interferon- $\gamma($ IFN- $\gamma)$ ) which enhance TGF- $\beta 1$-induced EMT $[118,119]$. IL-1 $\beta$ is a primary inflammasome cytokine produced by a variety of cells, including activated macrophages, dendritic cells, neutrophils, and epithelial cells and fibroblasts. It has been shown to contribute to the progression of adenoviral-induced pulmonary fibrosis and bleomycin-induced pulmonary fibrosis [120, 121].

Although several cytokines have been associated with IPF progression, the involvement of each individual immune cells is more complex. Recent studies have re-ignited the interest into the potential role of inflammation in lung fibrosis and have implicated t cells, neutrophils, alveolar macrophages, and other cells capable of releasing the important cytokine regulating mediators, potentially regulating IPF progression (IFN- $\gamma$, IL-2, TNF- $\beta$, IL-4, IL-5, IL-6, IL-9, IL10, IL-13) [122-128].

In addition to cytokines and soluble mediators, immune cells and fibroblasts can alternatively be activated by integrins, which can directly interact with the ECM [129]. Some integrins include $\alpha_{\| 1 b} \beta 3$ (fibrinogen receptor), $\alpha v \beta_{3}$ (vitronectin receptor), $\alpha_{2} \beta_{1}$ (collagen receptor), $\alpha_{5} \beta_{1}$ (fibronectin receptor), and $\alpha_{6} \beta_{1}$ (laminin receptor) [130]. This dissertation focuses mainly on collagen type I, because type I makes up $90 \%$ of all collagen in the body. The $\alpha_{2} \beta_{1}$ integrin is the main receptor that interacts with collagen $[131,132]$. Collagen has been found to induce M2 macrophages (Th2 response), feeding a feedback loop between fibroblasts and macrophages, promoting fibrotic responses to lung injury [133-135]. Expression levels of integrins also seems to play a role in fibroblastic response, with low $\alpha 2 \beta 1$ 
integrin expression enhancing the proliferation of fibroblasts from patients with IPF [136].

\subsection{Anti-Oxidants}

In the lung, another line of protection against the constant exposure to environmental chemicals and oxidants is a high concentration of anti-oxidants. When the tightly regulated balance of oxidants and anti-oxidants becomes altered, this state is referred to as oxidative stress [137]. Oxidant stress can be generated through many pathways including the generation of nitrogen and reactive oxygen species (ROS), alterations in Nox expression, and mitochondrial dysfunction [138]. ROS include superoxide, hydrogen peroxide and hydroxyl radicals, and cause unspecific damage to cells and extracellular matrix [139].

Anti-oxidants are the main line of defense against oxidative damage in the lung (Figure 6). These include superoxide dismutases (SODs), catalase, glutathione peroxidase, glutathione S-transferase, and thioredoxin. The SOD family catalyzes superoxide radicals into hydrogen peroxide and oxygen in a first order reaction. There are three mammalian SODs: cytosolic (SOD1), mitochondrial (SOD2), and extracellular (SOD3) [140-142]. The major intracellular SOD is SOD1, which is a 32-kD copper and zinc containing homodimer (also called Cu/Zn SOD) present in the cytoplasm and nucleus [143]. SOD2 (MnSOD) contains a manganese $93-\mathrm{kD}$ homotetramer and is synthesized in the cytoplasm to be translocated to the inner matrix of mitochondria $[141,144]$. SOD2 seems to play a 
role in neurodegenerative diseases [145]. SOD3 (EC-SOD) is the last mammalian SOD to be discovered and is primarily extracellular [142].

Acute and chronic forms of lung injury, including those associated with pulmonary fibrosis, are associated with oxidant stress [146]. BAL fluid from IPF patients showed increased levels of 8-isoprostane, a biomarker of oxidative stress [147]. Additionally, higher levels of oxidants (e.g. ELF myeloperoxidase, superoxide, hydrogen peroxide, iNOS) were detected in IPF patients, further suggesting a role for oxidant stress. [148-150].

With tobacco smoke being the main risk factor for IPF, some have postulated that cigarette smoke might promote tissue fibrosis through the induction of oxidant stress, yet the role of nicotine in this process is unclear [146]. In rats, nicotine has been shown to affect the oxidative state of the lung by increasing the activity of superoxide dismutase, an important protective mechanism in lung [151, 152]. Overexpression of superoxide dismutase was protective against fibrosis in the bleomycin model [153]. 


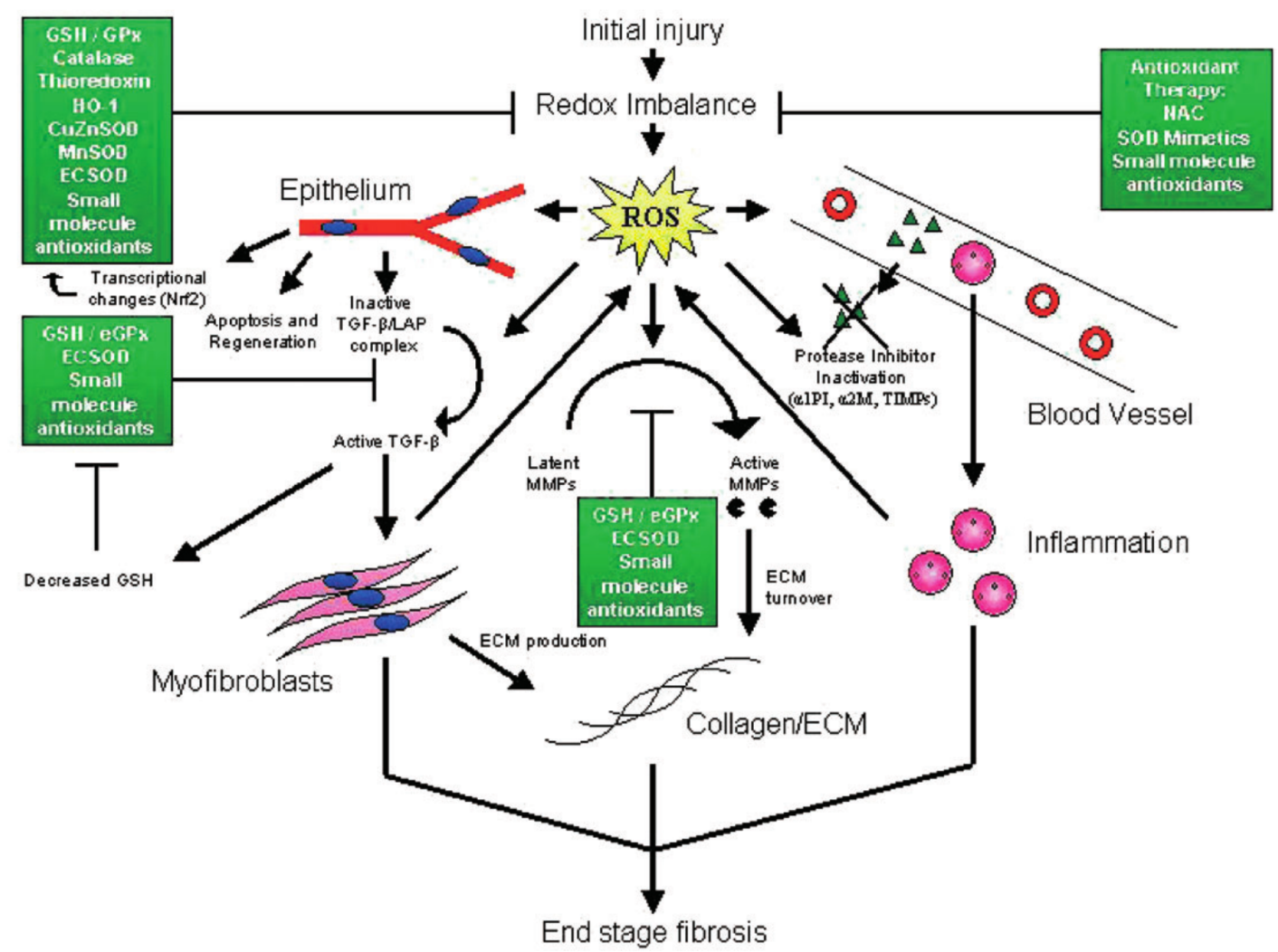

Figure 6. Oxidative Stress in IPF

Reprinted with permission from American Thoracic Society. [Am J Respir Crit Care Med] (Kinnula V.L. et Al., Oxidative stress in pulmonary fibrosis, 2005. 172(4): p. 417-422) Copyright 2005

Anti-oxidants are the main line of defense against oxidative damage in the lung. Exogenous and endogenous irritants in IPF create a redox imbalance, resulting in the production of ROS, which potentiate pulmonary fibrosis. Shown are endogenous antioxidants and the steps at which they can protect the lung from the effects of ROS. $\alpha 1 \mathrm{PI}=\alpha 1$-proteinase inhibitor; $\alpha 2 \mathrm{M}=\alpha 2$-macroglobulin; CuZnSOD = SOD1; ECSOD = SOD3; eGPx = extracellular glutathione peroxidase; $\mathrm{GPx}=$ glutathione peroxidase $; \mathrm{HO}-1$ = heme-oxygenase $1 ; \mathrm{MnSOD}=\mathrm{SOD} 2 ; \mathrm{TIMP}$ $=$ tissue inhibitor of metalloproteinases. 


\subsection{Modeling Pulmonary Fibrosis}

IPF occurs without cause in the majority of patients, making it difficult for science to develop relevant animal models that resemble human disease. Several models have been used to study pulmonary fibrosis, yet none can accurately recapitulate IPF. Fibrosing lung disease occurs naturally in a variety of animals including horses, cats, donkeys, and dogs (e.g. Westie Terriers) [154-158]. Although these are considered to have pulmonary fibrosis, the similarities between animal and human disease still need to be elucidated and, thus, they are difficult to use for research due to a myriad of issues including physiological differences, genetics, prevalence and cost [159].

More commonly, inducible models of pulmonary fibrosis are utilized. Bleomycin-induced pulmonary fibrosis is the most commonly used model to investigate the mechanisms that lead to tissue remodeling after lung injury [160]. Bleomycin has been used as an anti-cancer agent, as it is a glycopeptide antineoplastic agent derived from $\mathrm{Cu}(\mathrm{II})$ complexes from bacterium Streptomyces verticillus. Bleomycin is effective towards reducing or inhibiting the growth of neoplasias by degrading cellular DNA with single- and double-strand breaks. This is due to a DNA-binding and iron-binding site. Bleomycin forms a complex with iron, causing the iron to oxidize, therefore, reducing oxygen into free-radicals. The free-radicals cause single- and double-strand DNA breaks, leading to apoptosis, which is useful in causing damage to cancer cells. An unwanted side effect of 
bleomycin treatment, however, is the development of pulmonary fibrosis due to low abundance of the inactivating enzyme, bleomycin hydrolase, in the lung [161-163].

Physiologically, intratracheal bleomycin instillation elicits an acute pneumonitis characterized by the infiltration of the lung with immune cells within the first week. After the second week, inflammation subsides and the fibrotic stage of lung injury begins to form with the activation of tissue remodeling genes including fibronectin, collagens and TGF- $\beta$ [164-166]. This phase is characterized by modification of the original lung architecture and formation of pulmonary fibrosis (Figure 7).

Today, bleomycin is considered one of the best animal models to studying lung fibrosis due to several similarities with the human disease. IPF and bleomycininduced lung fibrosis share activation of (M2) macrophages, EMT, and microMRNA 21 induction [167-170]. One of the main drawbacks of the bleomycin model is the changing conditions between the different stages and the fact that the fibrosis is reversible [171]. Additionally, there is absence of hyperplastic type II AECs [172].

The bleomycin model has provided some insight into the role of tobacco exposure in pulmonary fibrosis, with cigarette smoke exposure enhancing alveolar architecture derangements in hamsters and guinea pigs [173-175]. However, cigarette exposure failed to enhance bleomycin-induced lung fibrosis in rats, but the exposure was not initiated till post bleomycin [176]. Thus, there appears to be a role for tobacco exposure in pulmonary fibrosis, but much remains to be elucidated. 


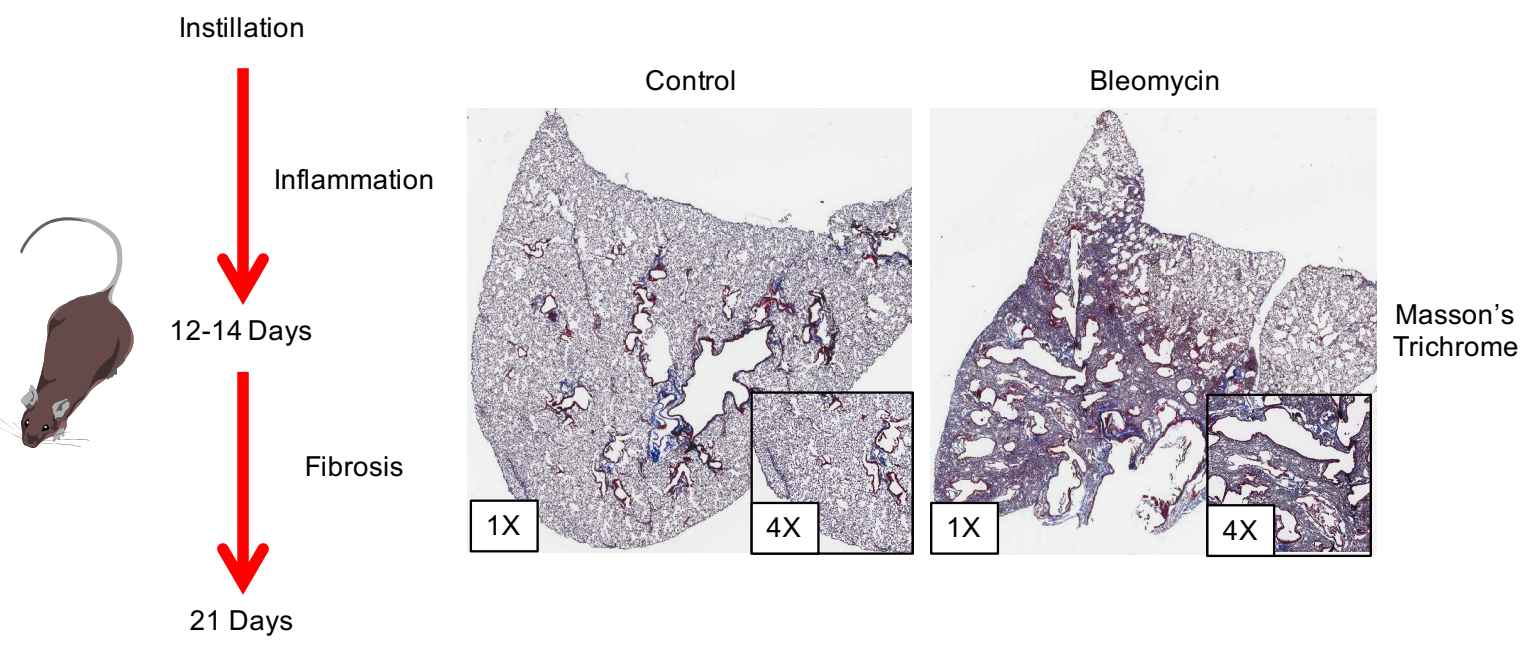

Figure 7. Bleomycin-Induced Model of Pulmonary Fibrosis

Vicary, Unpublished.

Bleomycin elicits an acute pneumonitis characterized by early infiltration of the lung with immune cells within the first week. In addition to inflammation, there is activation of tissue remodeling genes including fibronectin, collagens, and profibrotic TGF- $\beta$. Two weeks after bleomycin instillation, inflammation subsides and a fibrotic stage of lung injury begins to form after the second week. This phase is characterized by modification of the original lung architecture and replacement with fibrosis, shown here with Masson's tri-chrome staining. 


\subsection{Mechanobiology}

The lung is one of the few organs constantly expanding and contracting. How pulmonary fibrosis affects the mechanobiology of the lung and vice versa has only recently been investigated. Tissue forces were proposed to regulate tissue remodeling over a century ago, but it has taken modern research technology to begin to explore the relationship between mechanical forces and cellular behavior. Recent research has demonstrated that most eukaryotic cells can interact with the ECM and generate intracellular forces that affect cellular stiffness [177], cytoskeletal dynamics [178], cell migration [179-181], differentiation [182, 183] and drug response [184]. Cells can sense changes in the tensile strength and elastic recoil of the lung, which is determined by fibrillary proteins, such as collagens and fibronectins $[185,186]$.

During homeostasis, the turnover of ECM proteins varies greatly, which can alter the ECM stiffness [187]. Collagen fibers are estimated to remodel at 3-10\% per day, whereas elastin fibers, another ECM protein, are life-long, surviving approximately 74 years $[188,189]$. Disorders of matrix remodeling share a common paradigm with disease progression: provisional matrices formed in the context of injury emit signals to promote inflammatory responses and activate epithelial cells, provoking the production of connective tissue elements that lead to persistent and at times permanent matrix remodeling [190]. These changes in the rate of tissue remodeling likely plays an important role in pulmonary disease progression. 
Increased collagen deposition in IPF is associated with marked increases in lung stiffness, which has been shown to alter fibroblast morphology, proliferation, TGF- $\beta$ signaling, and myofibroblast activation [59, 191-194]. Collagen fibers affect matrix stiffness by strengthening protein-protein interactions and other physical characteristics such as resistance to proteolysis, emergence of neoepitopes, and enhanced cell-matrix interactions [195]. Collagen crosslinking can also play a major role in determining matrix stiffness, which feedbacks to increase additional collagen deposition in fibroblasts [196]. Enzymes can affect crosslinking, such as the lysyl oxidase family [195]. These various posttranslational modifications can result in significant changes of ECM moduli (protein ratios) and resultant pathology when normal remodeling functions are modified.

Traditionally, in vitro studies of cell-matrix interactions have used matrixcoated culture dishes of either solidary proteins such as collagen, or mixtures like Matrigel ${ }^{\circledR}$. These conditions do not recapitulate the in vivo 3D environment, however, as they lack characteristics such as the heterogeneity of lung tissue or 3D orientation of proteins. In vivo, cells protrude in all directions, often coming into contact with several ECM proteins at once. This is a limitation of culture systems, where cells react differently than cells in 3D environments [197-200]. Additionally, in vivo, cells are exposed to different ECM stress and strain forces due to the lung continuously expanding and contracting, exposing pulmonary cells (endothelial, epithelial and fibroblasts) to rhythmic stress and strain, which are necessary for cellular homeostasis. For example, airway epithelium exposed to cyclic stretching increases the production of surfactant and barrier function [201]. 
To study these 3D microenvironments in a more relevant system, the process of organ decellularization was developed, removing all cellular material, while retaining the ECM in its native 3D structure. Several processes have been published with variability in loss of proteins, growth factors, and matrix-associated molecules, depending on detergents uses, pressure applied, and the length of the process [202-204]. Decellularization allows measurement of the mechanical characteristics of the ECM with the development of physical tools such as the atomic force microscope (AFM) [205]. AFM is a high-resolution microscopy that uses a probe to touch the tissue surface and detect characteristics (force, imaging, sample manipulation) in the nanometer range (Figure 8) [206]. These strain/stress forces are expressed as elastic (Young's) modulus, which describes the force necessary to deform materials [207]. Different organs and tissues have different Young's modulus $(\mathrm{Pa})$ based of cell types and composition of ECM, among other factors, ranging from $50 \mathrm{~Pa}$ (blood plasma) to bone (100,000 Pa) (Figure 9) [208]. This is very different from tissue culture dishes which have Young's moduli of 24GPa [208]. Booth et. al. showed that after removal of cells, the normal human lung elastic modulus is 0.44 to $7.5 \mathrm{kPa}$ [59]. Several pulmonary diseases are associated with changes in lung Young's modulus, including emphysema having decreased stiffness, and fibrotic disorders such as IPF, having increased lung stiffness [59, 209, 210]. 


\subsection{Summary}

Affecting over 100,000 Americans and carrying a dismal diagnosis, IPF is a horrible disease. Diagnostic techniques have improved, but understanding the cause of IPF is still convoluted. This dissertation investigates the potential role of a transition matrix induced by nicotine exposure, and its effect on IPF formation and death associated with exposure to lung injury. Additionally, tracking of IPF is crucial to patient treatment, to which I investigate inclusion of obstructive airway measurement to improve IPF progression using spirometry values. Improving our understanding of the mechanisms by which tobacco promotes lung remodeling, will lead to the development of better tools for diagnostic tracking, care and treatment of these patients with this horrible condition. 


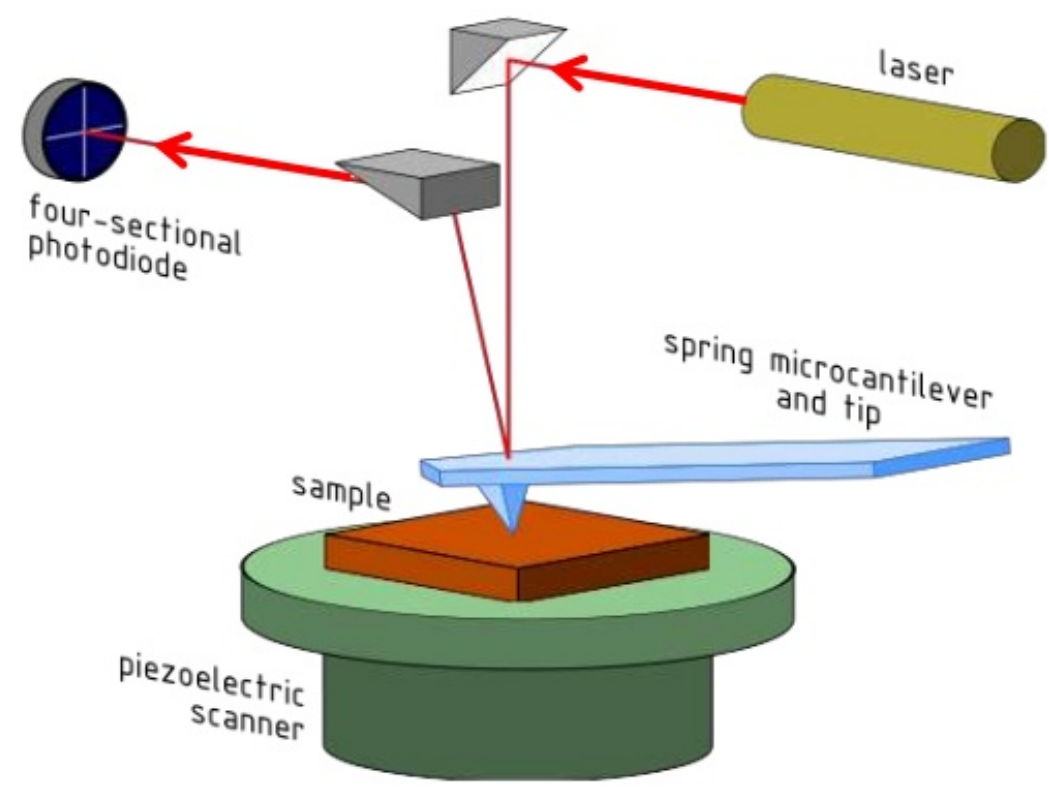

Figure 8. Mechanisms of Atomic Force Microscopy

Copyright Permissions not required for this type of use. (Bhattacharjee, J. Atomic Force Microscope: Fundamental Principles) http://www.slideshare.net/joybiitk/atomic-force-microscope-fundamental-principles (Wikipedia) https://en.wikipedia.org/wiki/Atomic-force_microscopy

Atomic force microscopy is a high-resolution microscope that uses a probe to touch the tissue surface and detect characteristics (force, imaging, sample manipulation) in the nanometer range using laser defection off a cantilever. These strain/stress forces are expressed as elastic (Young's) modulus, which describes the force necessary to deform a material. 


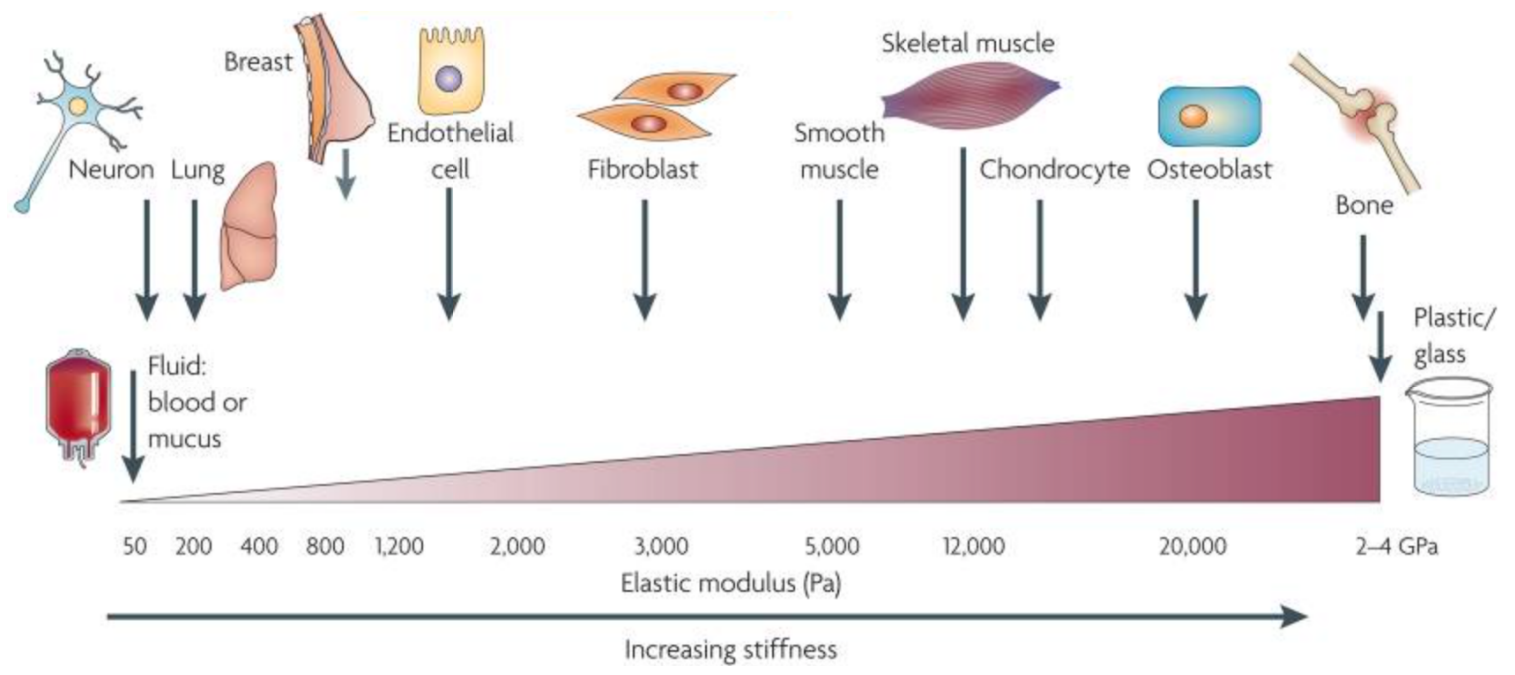

Figure 9. Lung Mechanobiology

Reprinted with permission from Nature Publishing Company. [Nature Review Cancer] (Butcher, D.T. et Al., A tense situation: forcing tumour progression, 2009. 9(2): p. 108-122) Copyright 2009

Substrate stiffness has been shown to alter fibroblast morphology, proliferation, TGF- $\beta$ signaling, and differentiation. Organs and tissues have different Young's modulus $(\mathrm{Pa})$ based of cells types, composition of ECM, among others ranging from $50 \mathrm{~Pa}$ (blood plasma) to bone $(100,000 \mathrm{~Pa})$. This is very different from tissue culture dishes which have Young's moduli of 2-4GPa. 


\section{CHAPTER III}

\section{MATERIAL AND METHODS}

\section{1 - Overview}

This dissertation consists of in vivo and in vitro experiments capable of investigating the complex interplay of nicotine and fibroblasts with the extracellular matrix. My experimental designs shed light on the potential role of nicotine and the $\alpha 7 \mathrm{nAChR}$ in the pathogenesis of pulmonary fibrosis.

\section{$\underline{2.2 \text { - Reagents }}$}

The mitogen-enhanced kinase-1 (MEK-1) inhibitor PD98059 was purchased from New England Biolabs, Inc. (Beverly, MA) and the $\alpha 7$ antagonist, MG-264, was purchased from Santa Cruz (Dallas, TX). Trypsin 10x was purchased from Corning Cellgro (Manassas, VA) and diluted to $2.5 \mathrm{X}$ with $\mathrm{dH}_{2} \mathrm{O}$. Mouse $\alpha 7 \mathrm{nAChR}$ siRNA, and control non-target siRNAs and Real-Time Quantitative PCR primers (QuantiTect Primer Assays, Qiagen, Valencia, CA) used to quantify mRNA levels by Real-Time RT-PCR were purchased from Qiagen. 
All other reagents were purchased from Sigma Chemical Company (St. Louis, MO) or Fisher Scientific (Pittsburgh, PA) unless otherwise specified.

\section{$\underline{2.3-\text { Cell Culture }}$}

Murine NIH3T3 fibroblast from the American-Type Culture Collection (CRL \#1658; Manassas, VA) and primary lung fibroblasts (between 3-10 passages) were harvested from wildtype control C57BL/6 or $\alpha 7$ nAChR deficient C57BL/6 mice (Jackson Laboratories, Bay Harbor, MA) and cultured in Dulbecco's Modified Eagle Medium (DMEM; Corning Cellgro), as previously described [77, 211]. The lack of $\alpha 7$ nAChRs in knock-out mice was verified by RT-PCR and Western Blot (Figure 12). U937, human monocytic cells permanently transfected with human IL-

$1 \beta$ gene promoter connected to a luciferase reporter gene were cultured in 400ug/ml HyClone geneticin G418 (Thermo Scientific, Waltham, MA) in RPMI1640 (Corning Cellgro). THP-1 monocytic cells transiently transfected with the luciferase gene connected to matrix metallopeptidase 9 (MMP-9) promoter were cultured in RPMI-1640 [212]. All media was supplemented with $10 \%$ fetal bovine serum (Atlanta Biologicals, Lawrenceburg, GA) and 1\% Antibiotic Antimitotic Solution (Corning Cellgro). Cells were grown in Heracell 150 incubators (Thermo Scientific) at $37^{\circ} \mathrm{C}$ and $5 \% \mathrm{CO}_{2}$. Cell viability was determined by Trypan Blue exclusion. 


\section{$\underline{2.4 \text { - Isolation and Detection of mRNAs by Reverse-Transcriptase }}$ Polymerase Chain Reaction}

RNA isolation and real-time PCR were performed in two ways. First was cells were lifted from wells with cell scrapers (Corning) and centrifuged (550 x g for 5 minutes). PBS were aspirated then $400-500 \mu \mathrm{L}$ of RNAzol B ${ }^{\text {TM }}$ (Tel-test Inc., Friendswood, TX) was added to the harvested cells and vortexed. Chloroformisoamyl alcohol ( $400 \mu \mathrm{L})$ was added, vortexed, and placed on ice for 15 minutes. Samples were spun at $4^{\circ} \mathrm{C}$ for 15 minutes at $17,500 \times$ g. mRNA was transferred to a new microfuge tube and $500 \mu \mathrm{L}$ cold isopropanol were added, mixed, and incubated on ice for $15-30$ minutes. Samples were spun at $4^{\circ} \mathrm{C}$ for 15 minutes at $17,500 \times \mathrm{g}$, and pellet washed with $95 \% \mathrm{EtOH}$ and then $70 \% \mathrm{EtOH}$. mRNA pellets were resuspended in $100 \mu \mathrm{L}$ of RNAse free $\mathrm{H}_{2} \mathrm{O}$ with $1 \mathrm{mM}$ EDTA. mRNA concentrations were determined by $\mathrm{OD}_{260} \times$ dilution factor $\times 40=\mathrm{ng} / \mu \mathrm{l}$ in crystal cuvettes using a GS-800 Calibrated Densitometer (Bio-rad). Reverse transcription was performed using iScript ${ }^{\mathrm{TM}}$ cDNA Synthesis Kit (Bio-rad) according to manufacturer's protocol.

PCR was performed using iTaq ${ }^{\mathrm{TM}}$ Universal SYBR $®$ Green Supermix (Biorad) [211]. Primer sequences are as follows: Mouse collagen type I forward (5'GTGCTGTTGGTGCTGCTG), reverse (5'-CAGGAGCACCAGCAATAC); $18 \mathrm{~s}$ forward (5'-GTGACCAGAGCGAAAGCA),

reverse ACCCACGGAATCGAGAAA); Mouse fibronectin forward primer (CTGTGACAACTGCCGTAG), reverse primer (CAGCTTCTCCAAGCATCG), 
probe

(ACCAAGGTCAATCCACA

C);

IL-1 $\beta$ forward

$(5$

GAGCACCTTCTTTTCC), reverse (5'-CTGGTGGAAGAAAAGG), probe; mouse $\alpha$-SMA forward primer (7ATGGAGTCAGCGGGCATC), reverse primer (AACTGGAGGCGCTGATCC), probe (AGCCACCGATCCAGACAG); mouse $\beta$ actin forward primer (ATGGATGACGATATCGCT), reverse primer (ATGAGGT AGTCTGTCAGGT), probe (GGATGGCTACGTACATGGCT); and $\alpha 7 \mathrm{nAChR}$ forward (5'-CTGCTGGGAAATCCTAGGCACACTTGAG or GACAAGACCGGCTTCCATCC), reverse CCTGGTCCTGCTGTGTTAAACTGCTTC) in a Labnet Multigene Gradient thermocycler (Edison, NJ) for PCR and Cepheid SmartCycler (Cepheid, Sunnydale, CA) for real-time PCR. Negative controls consisted of $\mathrm{dH}_{2} \mathrm{O}$ and mRNA without PCR agents. Values were normalized to $18 \mathrm{~S}$ or GAPDH and expressed as relative change vs. untreated mouse lung tissue.

The second method of isolation and detection isolated mRNA with QuickmRNA $^{\text {тм }}$ Micro- or Mini-Prep kits (Zymo Research, Irvine, CA) according to manufacturer's protocol. Real-time PCR conducted using Fast SYBR Master Mix (Applied Biosystems, Foster City, CA) using certified mouse primers for $18 \mathrm{~S}$ (Mm03928990_g1), $\beta$-Actin (Mm04394036_g1), GAPDH (Mm99999915_g1), Collagen Type 1 (Mm00801366_g1), Fibronectin (Mm01256744_m1), IL$1 \beta$ (Mm00434228_m1) SOD1 (Mm01344233_g1), TGF- $\beta 1$ (Mm01178820_m1), TNF- $\alpha$ (Mm00443258_m1) (Life Technologies, Carlsbad, CA) in a StepOnePlus ${ }^{\mathrm{TM}}$ real-time PCR system (Life Technologies). Negative controls consisted of $\mathrm{dH}_{2} \mathrm{O}$ 
and mRNA without PCR agents. Values were normalized to $18 \mathrm{~S}, \beta$-Actin, or GAPDH and expressed as relative change vs. untreated samples.

\section{5 - Protein Detection via Western Blotting}

Western blots were performed as previously described [77, 211]. Samples were isolated using western homogenization buffer $(50 \mathrm{mM} \mathrm{NaCl}, 50 \mathrm{mM} \mathrm{NaF}, 50$ mM NaP2O7-10 H2O, 5 mM EDTA, 5 mM EGTA, 2 mM Na3VO4, 0.5 mM PMSF, 0.01\% Triton X-100, $10 \mu \mathrm{g} / \mathrm{ml}$ leupeptin, $10 \mathrm{mM}$ HEPES, $\mathrm{pH}$ 7.4), and sonicated for 5 seconds using Sonifier 450 (Branson, Danbury, CT). Protein concentrations were determined using Bradford reagent (Sigma) readings in DU-800 Spectrophotometer (Beckman Coulter, Brea, CA). Gels were either 10\% (denaturing) or $8 \%$ (native) acrylamide gels (Bio-rad, Hercules, CA) run in a mini trans-blot system (Bio-rad). Samples were heated to $95^{\circ} \mathrm{C}$ for 5 minutes and spun for 10 minutes at $17,500 \times \mathrm{g}$. Gels were run for 2 hours at 125 volts using a Powerpack HC power supply (Bio-rad).

Protein was transferred to Protran Nitrocellulose transfer membrane (Whatman), between 4 pieces of extra thick western blotting filter paper (Thermo Scientific), soaked in Pierce Western Blot Transfer Buffer (Thermo Scientific), then transferred for 2 hours at 25 volts in Trans-Blot SD semi-dry transfer cell (Bio-rad). Membranes were agitated in wash buffer $(3 \times 10$ minutes $)$ and then incubated in $5 \%$ Bovine Serum Albumin or $5 \%$ non-dry fat milk blocking buffer for 1 hour. Blots were incubated with primary polyclonal antibody against either GAPDH (Sigma; 
1:1000 dilution), Collagen type I (Abcam, Cambridge, MA; 1:10000; native, nonreducing gel), p-Smad3 (Rockland Immunochemicals, Gilbertsville, PA; 1:2000), p-Erk 1\&2 (Cell Signaling, Beverly, MA; 1:1000), total Smad3 (Upstate Cell Signaling, Lake Placid, NY; 1:1000), total Erk (Cell Signaling; 1:1000), and $\alpha 7$ nAChR (Sigma; 1:500) overnight at $4^{\circ} \mathrm{C}$. Membranes were agitated in wash buffer ( $3 \times 10$ minutes) before incubation in $2^{\circ}$ antibody goat anti-rabbit IgG (Sigma; $1: 20,000)$ for 1 hour at room temperature.

Non-florescent blots were incubated with Amersham ECL Western Blotting Detection Reagents (GE Healthcare, Little Chalfont, UK) for 5 minutes and exposed to Genemate Blue Basic Autorad film (Bioexpress, Kaysville, UT) for up to 1 hour. Protein densitometry was completed using GS-800 Calibrated Densitometer (Bio-rad).

Florescent lots were incubated with secondary goat anti-rabbit IgG (HRPconjugated) (Li-CORE, Lincoln, NE) (1:20000). Membranes were agitated in wash buffer ( 3 x 10 minutes). Florescent blots read on a LI-COR Odyssey CLx imaging system and analyzed in Image Studio (LI-COR).

\section{6 - Cell Viability (Proliferation) Assay}

Wildtype or transfected primary lung fibroblasts $\left(1 \times 10^{4}\right.$ cells $\left./ \mathrm{ml}\right)$ were added to 96 -well tissue culture plates and incubated at $37^{\circ} \mathrm{C}$ for 24 hours in COMPLETE ${ }^{\text {TM }}$ Serum-Free/Low-Protein Medium (Corning Cellgro), then for up to 72 hours with nicotine $(50 \mu \mathrm{g} / \mathrm{ml})$ in the presence or absence of the MEK1 inhibitor, 
PD98059 $(50 \mu \mathrm{M})$. Afterwards, the luminescence of viable cells was detected using Cell Titer-Glo Luminescent Cell Viability Assay Kit (Promega) in a Luminoskan Ascent Luminometer (Beckman Coulter) according to the manufacturer's instructions.

\section{7 - Matrix Deposition and IL-1 $\beta$ Measurement}

Fibroblasts were cultured for 24 hours then treated with nicotine $(50 \mu \mathrm{g} / \mathrm{ml})$, PD98059 $(50 \mu \mathrm{M})$, and/or MG-264 $(10 \mu \mathrm{M})$ in DMEM (Cellgro) and retained in culture in 6 well Costar Cell Culture plates (Corning) for 120 hours. Afterwards, the fibroblasts were eliminated by osmotic lysis and the remaining matrix-coated dishes were washed thoroughly [213]. Cells were washed once with PBS containing $1 \mathrm{mM}$ EDTA (Solution A), then treated for 30 minutes at $4^{\circ} \mathrm{C}$ with the solution $\mathrm{B}\left(0.25 \mathrm{M} \mathrm{NH}_{4} \mathrm{OH}, 1 \mathrm{mM}\right.$ EDTA, $1 \mathrm{mM}$ PMSF). The cells were washed 2 additional times with solution $3 \mathrm{~A}$, and then treated for 15 minutes at $4^{\circ} \mathrm{C}$ with solution $\mathrm{C}(1 \mathrm{M} \mathrm{NaCl}$ in $50 \mathrm{mM}$ Tris (pH 7.4), $1 \mathrm{mM}$ EDTA, $1 \mathrm{mM}$ PMSF). Lastly, the culture plates were washed once with solution A. Isolated matrices were stored at $4^{\circ} \mathrm{C}$ with PBS.

Human monocytic U937 cells permanently transfected with the human IL$1 \beta$ gene promoter fused to a luciferase reporter gene were incubated in RPMI1640 on matrix-coated plates for 24 hours [214]. Inhibition was achieved by pretreatment of mouse $\lg (1: 100$, Sigma) or anti- $\alpha 2 \beta 1$ integrin (1:100, Abcam) antibody for 1 hour followed by culturing with the matrix-coated plates 24 hours. 
Afterwards, the luminescence of viable cells was detected using Cell Titer-Glo Luminescent Cell Viability Assay Kit (Promega).

\section{$\underline{2.8 \text { - Superoxide Dismutase Activity Assay }}$}

Activity of superoxide dismutases was determined using an in vitro assay as previously published [215]. The quantity of SOD required to produce $50 \%$ inhibition of the rate of reduction of cytochrome $\mathrm{c}$ under specified conditions is defined as 1 unit. Samples were collected in $600 \mu \mathrm{L}$ cold PPS solution (50 mM potassium phosphate, $0.1 \mathrm{mM}$ EDTA, $\mathrm{H}_{2} \mathrm{O}, \mathrm{pH} 7.8$ ) and homogenized thoroughly and spun for 30 minutes at $14,000 \times$ g. In a GS-800 Beckman Coulter spectrometer, the control reaction was determined adding Ferricytochrome C (Cytochrome C) $0.1 \mathrm{mM}$ (Sigma \#C7752), Xanthine (Sigma \#X4002), and Phosphate buffer $(800 \mu \mathrm{L})$ into $1 \mathrm{~mL}$ plastic cuvette with light path $1 \mathrm{~cm}$ at $25^{\circ} \mathrm{C}$. Reaction initiated by adding $10 \mu \mathrm{L}$ Xanthine Oxidase (Sigma \#X4875) and the sample and read at $550 \mathrm{~nm}$ every 30 seconds for 300 Seconds.

\section{$\underline{2.9-\text { Murine Housing }}$}

Wildtype or $\alpha 7$ nAChR deficient C57BL/6 mice (female, 8-12 weeks; Jackson Laboratories) were housed on a 12-hour light cycle in a pathogen-free barrier facility accredited by the Association for Assessment and Accreditation of 
Laboratory Animal Care. The University of Louisville's Institutional Animal Care and Use Committee approved all animal studies.

\section{$\underline{2.10-\text { Murine Nicotine Exposure }}$}

Wildtype and $\alpha 7$ nAChR knockout C57BL/6 mice (female, 8-12 weeks) were fed a normal diet and exposed to nicotine in their drinking water $(100 \mu \mathrm{g} / \mathrm{ml})$ for 8 weeks as previously described by Rowell et al. [216, 217]. Per Rowell's research, mice received an average of $28.6 \mathrm{mg} / \mathrm{kg}$ of nicotine a day with a plasma nicotine concentration approximately $40 \mathrm{ng} / \mathrm{ml}(0.21 \mu \mathrm{M})$, which is comparable to what has been seen in humans. In vitro doses of nicotine $(1-100 \mu \mathrm{g} / \mathrm{ml})$ used was chosen based on previous experiments in the lab and published literature [77].

\section{$\underline{2.11 \text { - Murine Cigarette Smoke Exposure }}$}

In collaboration with Douglas Arenberg at the University of Michigan, mice were exposed to cigarette smoke with an automated smoking machine (JaegerBaumgartner CSM) as previously described [218-220]. The machine draws a puff of smoke in two seconds at a flow of 1.02 liters/min, once each minute. The smoke was diluted with medial grade air at 3 liters/min and injected into a sealed 22-gallon fish tank containing up to 5 Mico-isolator mouse cages (tops removed). This mixture yields a particulate content of $250 \mathrm{mg} / \mathrm{cubic}$ meter. Mice were exposed 4 hours/day 5 days/week for up to 6 months. Exposures are well tolerated using this 
system for up to one year with acceptable levels of carboxyhemoglobin.

\subsection{2 - Murine Bleomycin-Induced Injury}

Bleomycin-induced lung injury was performed as previously described [70]. Mice received either bleomycin (1.0 or $2.0 \mathrm{U} / \mathrm{kg}$ ) (Sigma) in phosphate-buffered saline (PBS) or vehicle (PBS) alone intratracheally. Mice were euthanized at 12 or 21 days following injury. Mice were euthanized followed by mRNA and protein isolation along with en bloc isolation of the lungs for analysis.

\section{$\underline{2.13 \text { - Murine Bronchoalveolar Lavage (BAL) }}$}

Bronchoalveolar lavage (BAL) was performed as previously described [212]. Cells obtained from BAL were plated for 1 hour at $37^{\circ} \mathrm{C}$ and the supernatant collected. Supernatant $(100 \mu \mathrm{L})$ was cultured on NIH3T3 fibroblasts or THP-1 monocytes for 24 hours. The supernatants from culture were utilized in fibronectin

or MMP-9 gene transcription assays using Luciferase Assay Reagent (Promega) as previously described [212].

\subsection{4 - Murine Histological Analysis}

Mice were euthanized by exsanguination followed by en bloc isolation of the lungs which were inflated at standard pressure, fixed in formalin, paraffin- 
embedded, and sectioned (5 $\mu \mathrm{m}$ ) using JUNG RM2055 microtome (Leica, Buffalo Groce, IL), then transferred onto Colorfrost microslides (VWR Sciences, Radnor, PA) for histological analysis. Lung sections were stained using Weigert's iron hematoxylin for 10 minutes, rinsed in $\mathrm{dH}_{2} \mathrm{O}$, then treated with Biebrich scarlet-acid fuchsin solution for 10 minutes. The slides were washed in $\mathrm{dH}_{2} \mathrm{O}$, then transferred to aniline blue stain for 30-60 minutes (Masson Tri-chrome Staining Kit, RichardAllan Scientific, Kalamazoo, MI). For Sirius Red/Fast Green staining, slides were treated with 5\% Sirius Red (Polysciences Inc, Warrington, PA) and then were Fast Green (Achros, New Jersey) saturated with picric acid for 30 minutes each. Lung microscopy pictures were taken with XL Core EVOS microscope (Life Technologies, Carlsbad, CA). The tri-chrome slides were blindly graded on their intensity of collagen staining by 6 investigators based on the Ashcroft score as previously described [221].

\subsection{5 - Protein Detection via Immunohistochemistry}

Immunohistochemistry was performed as previously described [77, 222]. Application of primary antibodies against fibronectin, DAPI, IgG, or IL-1 $\beta$ (Abcam, 1:100 dilution) were incubated at $4{ }^{\circ} \mathrm{C}$ overnight. HRP-conjugated secondary antibodies were applied (Sigma), and the specific staining was visualized with Vectastain ABC Kit (Vector Labs, Burlingame, CA). Lungs of smoked and nicotinetreated mine were processed and incubated with fibronectin antibodies Afterwards, the tissue sections were de-identified and the sections were scored for fibronectin 
expression by two investigators blinded to treatment groups.

\section{$\underline{2.16 \text { - Murine Lung Decellularization }}$}

Based off previously published method [59], mice were euthanized by $\mathrm{CO}_{2}$. Lung and heart were isolated en bloc. All reagents were made fresh for each use. Lungs were perfused in a $50 \mathrm{~mm}$ cell culture dish by injecting $10 \mathrm{cc}$ of DI solution $\left(\mathrm{dH}_{2} \mathrm{O}\right.$ with $1 \%$ Antibiotic Antimitotic Solution (Corning Cellgro)) into the right ventricle of the heart. Three-cc syringe with 19-gauge needle were filled with DI solution and inserted into trachea and secured with 2 silk suture knots. Lungs were inflated with DI solution with needle left in trachea. All perfusion steps were performed by injecting $3 \mathrm{~mL}$ intracardiac and intratracheal samples of each of the solutions and the lungs were allowed to recoil and expel any excess fluid. Perfusions consisted of $0.1 \%$ Trition X-100 (Sigma), 2\% Na deoxycholate (Fisher), $1 \mathrm{M} \mathrm{NaCl}$ (Fisher), and DNAse solution (DNAse I (Sigma), $30 \mu \mathrm{g} / \mathrm{ml}$ in $1.3 \mathrm{mM}$ $\mathrm{MgSO}_{4}$ and $2 \mathrm{mM} \mathrm{CaCl}$ ). Perfusions of each solution were followed by incubations at $4^{\circ} \mathrm{C}$ on a rocker with gentle stirring for minimum of 1 hour each, and solution change were performed in the tissue culture hood. After each incubation, the lungs were flushed with DI solution before the next perfusion. Once the decellularization process was complete, the lungs were stored in $50 \mathrm{~mL}$ conicals containing PBS with $100 \mu \mathrm{g} / \mathrm{ml}$ Antibiotic Antimitotic Solution (Corning Cellgro) and $2.5 \mu \mathrm{g} / \mathrm{ml}$ Amphotericin B (Lonza) until embedding. 
Lungs are embedded in 6\% Seaprep Ultra Low Agarose (LONZA). Agarose was prepared on hot plate with stir rod and allowed to mix at medium heat until fully dissolved. Once melted, agarose was placed in a $55{ }^{\circ} \mathrm{C}$ degree water bath until use. Lung was dried on paper towel and dabbed dry. One and half $\mathrm{mL} 6 \%$ ultra-low melt agarose was injected into the trachea and heart and the organs were cut out and the lung dissected into separated lobes, placed into embedding molds, and covered with additional agarose. The embedded tissue was stored in a sealed container with a humidification agent.

Lung sections cut on Vibratome (Leica VT 1200, Wetzlar, Germany) at 0.2 $\mathrm{mm} / \mathrm{s}$ and $500 \mathrm{~nm}$. Loctite (Office Max, Cat\# A11363588) was used to glue sample to cutting platform. Cut samples were stored in DI solution in $50 \mathrm{~mL}$ conical. The agarose was melted by adding the tubes into a water bath set to $50^{\circ} \mathrm{C}$. To sterilize the slices before use, the DI solution was changed to $M Q$ water plus $0.18 \%$ peracetic acid (Pfalz \& Bauer) plus $4.8 \% \mathrm{EtOH}$ over night or the weekend at $4^{\circ} \mathrm{C}$. Afterwards, the sterilizing solution was decanted and cold sterile filtered water was added and rocked in cold room.

\section{$\underline{2.17-\text { Atomic Force Microscopy }}$}

Matrix stiffness was measured by atomic force microscopy (AFM). Stress is the magnitude and direction of the force on the cell and strain is the magnitude and direction of distortion (force/area) [223]. AFM is a very high-resolution type of scanning probe microscopy that utilizes a cantilever as a probe to characterize the 
surface of a sample. When the tip of the cantilever contacts the sample, forces between the tip and the sample lead to a deflection of the cantilever. The deflection is measured based on the displacement of a laser using an array of photodiodes.

Based on a previously published method [165]. Samples were tested with MFP-3D-Bio AFM Microscope (Asylum Research, Santa Barbra, CA) using cantilevers with a $0.03 \mathrm{~N} / \mathrm{m}$ spring and $10 \mu \mathrm{m} \mathrm{SiO}{ }_{2}$ Sphere tip (Novascan, Ames, IA). A force map ( $80 \mu \mathrm{M}$ scan size) was generated with a $16 \times 16$ spot test pattern for a total of 256 points. Each force plot was conducted with a velocity of $2 \mu \mathrm{M} / \mathrm{s}$ and a trigger point of $500 \mathrm{nM}$. Young's modulus was determined using a Hertz fit model, setting the tip poisson of 0.19 and modulus of $68 \mathrm{GPa}$, and a sample poisson of 0.4 . The upper limit of the calculation region was set at $75 \%$ of the curve. Data gathered and analyzed using Asylum Modified (Version 14) Igor Pro software (6.22A WaveMetrics Inc., Portland, OR).

\section{$\underline{2.18-\text { Statistical Evaluation }}$}

All experiments were repeated using at least 3 samples. Means plus standard deviations of the means were calculated for all experimental values. Significance was assessed by $p$-values $<0.05$, which were obtained using twotailed t-tests in Microsoft Excel (Redman, WA) or 2-way ANOVA with Bonferroni posttest in Graphpad Prism 6 (Graphpad Software, La Jolla, CA).

\section{$\underline{2.19}$ - PANTHER Trial Data}


The datasets for the Prednisone, Azathioprine, N-acetyl cysteine in IPF (PANTHER) Trial were obtained from NIH-NHLBI-sponsored Biologic Specimen and Data Repository Information Coordinating Center (BioLincc). This study was designed and conducted by the Idiopathic Pulmonary Fibrosis Clinical Research Network (IPFnet). The trial lasted 60 weeks and participants received a combination of prednisone, azathioprine, and NAC (combination therapy), NAC alone, or placebo in a 1:1:1 ratio [224] (ClinicalTrials.gov number, NCT00650091). At the end of the trial, increased risks of death and hospitalization were observed in patients treated with a combination of prednisone, azathioprine, and NAC, but no difference in FVC was detected. The database provided 240 patients for analysis. Because no difference in FVC was detected between treatment and placebo groups in the trial, all 85 patients (43 placebo/42 treatment) who achieved $>10 \%$ decline in the FVC during the study were included in statistical analysis. Spirometry values were collected every 15 weeks during the trial period of 60 weeks. Secondary outcomes include the frequency of re-hospitalizations and time of death.

\section{$\underline{2.20 \text { - PANTHER Trial Statistical Analysis }}$}

The analysis was completed in $\mathrm{R}$ (Version 3.2.4) [225]. A $10 \%$ loss in either

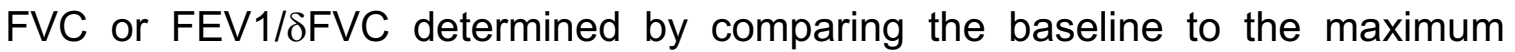
reading from the $15,30,45$, or 60 -week follow-up. Descriptive statistics were ran 
comparing placebo and treatment groups. Chi-squared or Fisher's exact tests were used as appropriate to test for differences between categorical variables. Wilcoxon rank sum tests were used to test for differences in continuous variables. The Stuart Maxwell Marginal Homogeneity Test (an extension of McNemar's test) was used to assess differences timing of loss between FVC and FEV1/אFVC. Receiver

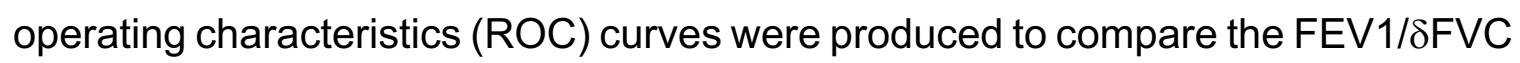
ratio versus FVC alone in regards to sensitivity and specificity as a predictor of death and re-hospitalization.

\subsection{1 - PANTHER Trial Human Subject Protection}

This study was approved by the University of Louisville Institutional Review Board (IRB) - Human Subjects Protection Program Office (HSPPO). 
CHAPTER III

\begin{abstract}
THE EFFECTS OF NICOTINE ON LUNG FIBROBLAST PROLIFERATION AND COLLAGEN EXPRESSION/DEPOSTION IN VITRO AND IN VIVO, AND THE CHOLINERGIC RECEPTORS RESPONSIBLE FOR THESE EFFECTS
\end{abstract}

\title{
$\underline{3.1 \text { Introduction }}$
}

Tobacco is considered the single greatest cause of preventable death in the world [226]. Chronic tobacco exposure is associated with the development of chronic lung disorders like chronic obstructive pulmonary disease and idiopathic pulmonary fibrosis (IPF), and is the main cause of lung cancer globally [226]. Tobacco smoke contains more than 4000 chemicals, which interact with a multitude of molecules and pathways, preventing the effective and safe targeting of a single mechanism of action with significant therapeutic benefit. A larger effect of tobacco smoke inhalation is the induction of inflammation, a process characterized by the release of soluble mediators, oxidant stress, and the recruitment of inflammatory cells into tissue [227]. This promotes tissue remodeling, including alterations in lung structure and function, and oncogenesis [228]. 
Due to nicotine's well known addictive effects, attention has turned to this plant derived alkaloid which represents $\sim 0.6-3.0 \%$ of the dry weight of tobacco. Recent studies have unveiled the effects of nicotine on lung development [34, 35] and on inflammatory processes [229]. This is possible because of the existence of nicotinic receptors in lung tissue capable of signal transduction [79]. These receptors, termed nicotinic acetylcholine receptors ( $\mathrm{AAChRs),} \mathrm{are} \mathrm{multimeric}$ acetylcholine-triggered channel proteins that form homomeric and heteromeric $\alpha$ and $\beta$ chain pentameters. The $\alpha 7 \mathrm{nAChR}$ is the most abundant homomeric receptor form, composed of five $\alpha 7$ subunits. In developing primate lungs, $\alpha 7$ nAChRs were detected in airway epithelial cells, around large airways and blood vessels, free alveolar macrophages, alveolar type II cells, and pulmonary neuroendorcrine cells [34]. Additionally, increased $\alpha 7 \mathrm{nAChR}$ expression has been shown with nicotine administration in bronchial epithelial and endothelial cells as well being implicated in regulation of inflammation and cancer [77, 92-94].

Our laboratory has previously demonstrated that nicotine stimulates lung fibroblasts to express fibronectin through induction of the MEK-1/Erk pathway [77]. Fibronectin is a matrix glycoprotein, which is highly expressed in injured tissues, and is considered a sensitive marker of tissue injury and activation of tissue remodeling [60,61]. In an injured lung, fibronectin is deposited over denuded basement membranes where it is thought to support the migration of alveolar epithelial cells during repair [61]. The excessive deposition of fibronectin, however, has been hypothesized to promote disrepair [70]. Human studies also show increased fibronectin content in the bronchoalveolar lavage fluid of smokers [75]. 
However, nicotine-induced fibronectin expression and deposition are not sufficient to alter overall lung architecture and to date there are no data suggesting that nicotine, alone, promotes significant lung tissue remodeling.

In this study, nicotine is shown to stimulate lung fibroblasts to express collagen type I. This fibrillar collagen is another extracellular matrix component highly expressed in tissues during injury and repair, and its expression signals activation of tissue remodeling. Collagen type $\mathrm{I}$ is the most abundant form of collagen in the human body and is present in connective tissue throughout the body including tendon, ligament, skin, and lung tissue. Each rope-like procollagen molecule is made up of three chains: two pro- $\alpha 1$ (I) chains, which are produced from the COL1A1 gene, and one pro- $\alpha 2$ (I) chain, which are produced from the COL1A2 gene. After processing, the resulting mature collagen molecules arrange themselves into long, thin fibrils. Individual collagen molecules are then crosslinked to one another within these fibrils thereby forming strong collagen fibrils. Studies performed in vivo confirmed nicotine induction of collagen type I without changes in overall lung architecture in lung matrix. Also, we found that nicotinetreated fibroblasts produce a collagen-containing matrix capable of stimulating monocytic cells to produce the pro-inflammatory cytokine IL-1 $\beta$ in vitro.

Together, these observations suggest that nicotine stimulates alterations in the relative composition of the lung extracellular matrix favoring fibronectin [77] and collagen type I (this report) expression without altering the overall tissue architecture of the lung. These subtle changes may render the host susceptible to excessive tissue damage after injury. 


\subsection{Results}

\section{Nicotine stimulates the expression of collagen type I}

While exploring the effects of nicotine on lung cells, we found that nicotine stimulated the expression of collagen type I in lung fibroblasts. As depicted in Figure 10, nicotine induced the expression of collagen type I mRNA in a dosedependent fashion (Figure 10). Maximum mRNA expression was observed at 50 $\mu \mathrm{g} / \mathrm{ml}$ of nicotine. Twenty-four hours after nicotine stimulation, increased collagen type I protein was detected in cell extracts (Figure 10). Nicotine also stimulated collagen type I mRNA and protein expression up to $72 \mathrm{~h}$ (Figure 11).

\section{Role of $\alpha 7 \mathrm{nAChRs}$ in collagen expression and cellular proliferation}

We evaluated the expression of $\alpha 7 \mathrm{nAChR}$ in $\alpha 7 \mathrm{KO}$ and wildtype mice (Figure 12). RT-PCR verified the absence of $\alpha 7 \mathrm{nAChRs}$ in the $\alpha 7 \mathrm{KO}$ mice. We then evaluated nicotine-induced collagen type I mRNA expression in primary lung fibroblasts harvested from $\alpha 7 \mathrm{KO}$ animals. Nicotine failed to stimulate collagen type I expression in $\alpha 7 \mathrm{KO}$ cells (Figure 12). Similarly, we found nicotine did not induced collagen type I protein expression in $\alpha 7 \mathrm{KO}$ fibroblasts. (Figure 12). Nicotine also stimulated the proliferation of lung fibroblasts and this effect was also through $\alpha 7$ nAChRs. As depicted in Figure 13, nicotine-induced proliferation was inhibited at 48 and $72 \mathrm{~h}$ by siRNA silencing of $\alpha 7 \mathrm{nAChRs}$. Additionally, the mitogenic effects of nicotine were inhibited by PD98059, a MEK-1/ERK inhibitor (Figure 14). 
A

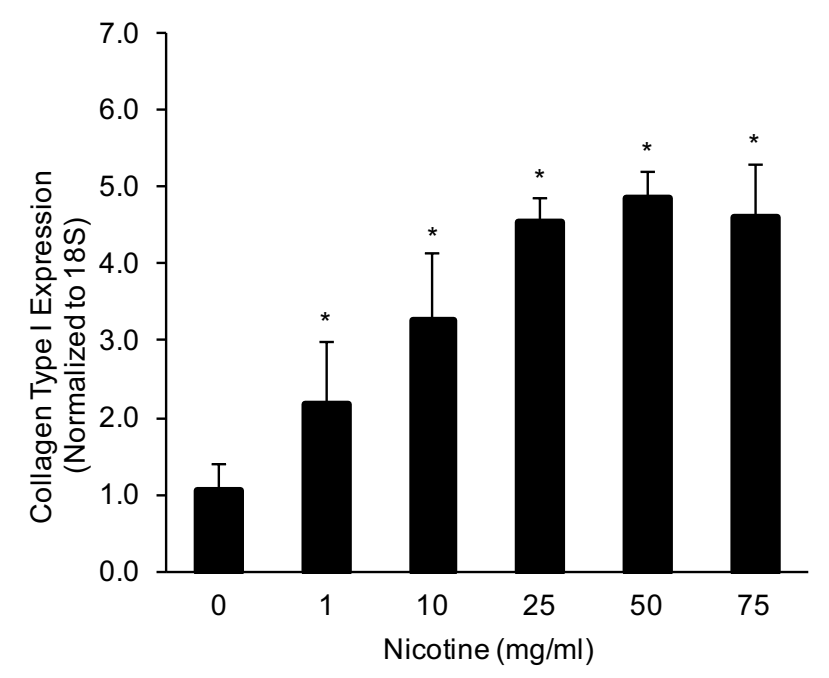

B

Collagen (I) Protein Expression
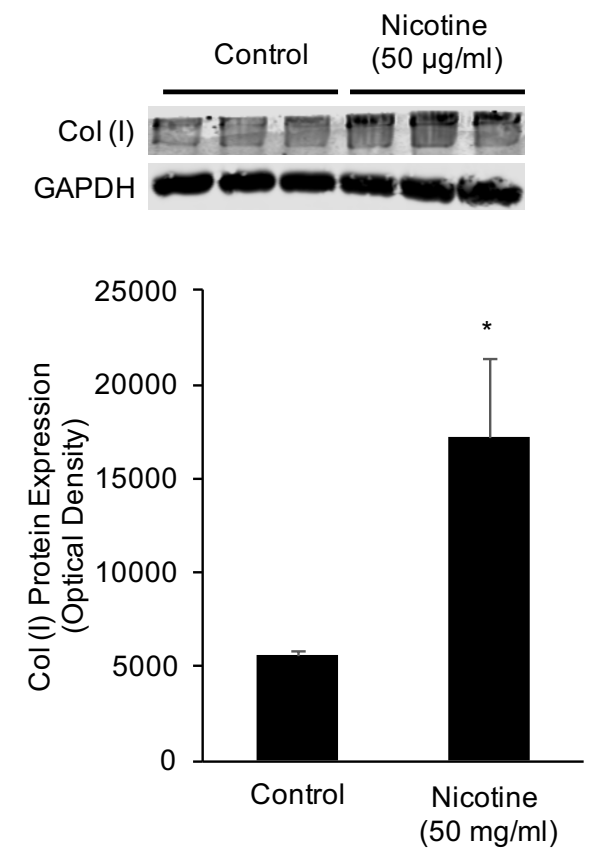

Figure 10. Nicotine Stimulates Collagen Type I mRNA and Protein Expression

Vicary et. Al. 2016

(A) Primary lung fibroblasts $\left(1 \times 10^{6}\right.$ cells/6 well) were treated with nicotine (1-75 $\mu \mathrm{g} / \mathrm{ml}$ ) for 24 hours. Real-time PCR reactions were performed using mouse collagen type I or $18 \mathrm{~S}$ primers. Note that nicotine induced a dose-dependent increase in collagen type I mRNA expression. mRNA levels were normalized to 18 S and collagen type I levels compared to untreated controls. (B) Nicotine-treated fibroblasts were subjected to Western blot analysis using anti-collagen type I antibody or GAPDH, followed by secondary goat anti-rabbit lgG (IRDye $\left.{ }^{\circledR}\right)$. Protein bands were separated by native (collagen type 1) or SDS-PAGE (GAPDH) gel electrophoresis ( $8 \%$ ) and quantified by densitometry. Collagen type I levels were elevated in the presence of nicotine $(50 \mu \mathrm{g} / \mathrm{ml})$. Significance was assessed using $p$ values $<0.05$ obtained by two-tailed t-test. 
A

Collagen (I) mRNA Expression

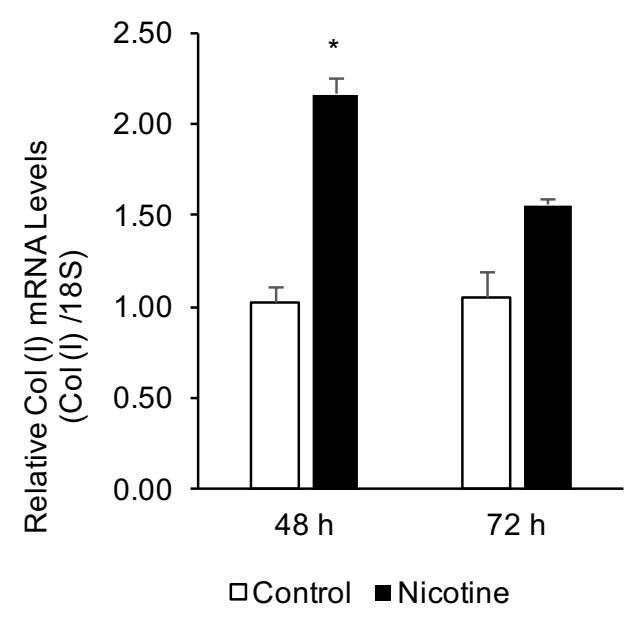

B

Collagen (I) Protein Expression

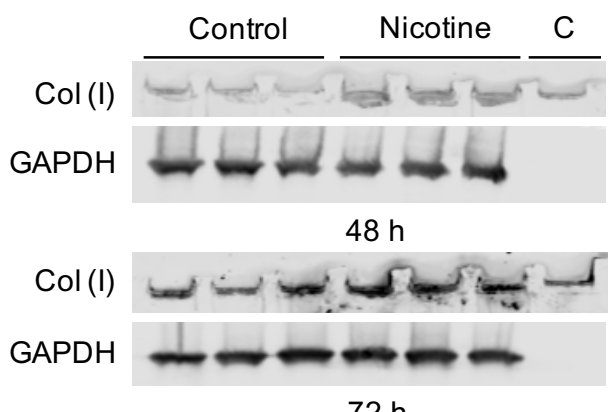

$72 \mathrm{~h}$

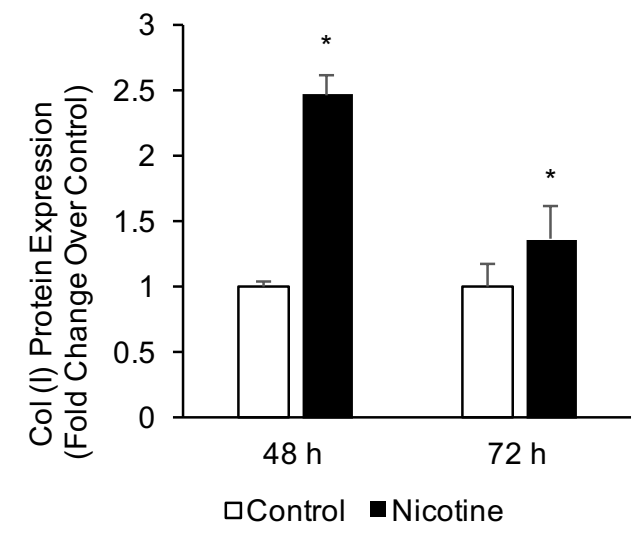

Figure 11. Nicotine Stimulates Collagen Type I mRNA and Protein Expression at 48 and 72 Hours

Vicary et. Al. 2016

(A) Primary lung fibroblasts were treated with nicotine $(50 \mu \mathrm{g} / \mathrm{ml})$ for up to 72 hours. Nicotine induced a significant increase in collagen type I mRNA levels at 48 hours. (B) Fibroblasts were treated with nicotine $(50 \mu \mathrm{g} / \mathrm{ml})$ for up to 72 hours. Collagen type I protein was increased at 48 and 72 hours by Western blot analysis. C: Purified rat-tail collagen. All experiments repeated at least 3 times. Significance was assessed using $p$ values $<0.05$ obtained by two-tailed t-test. 


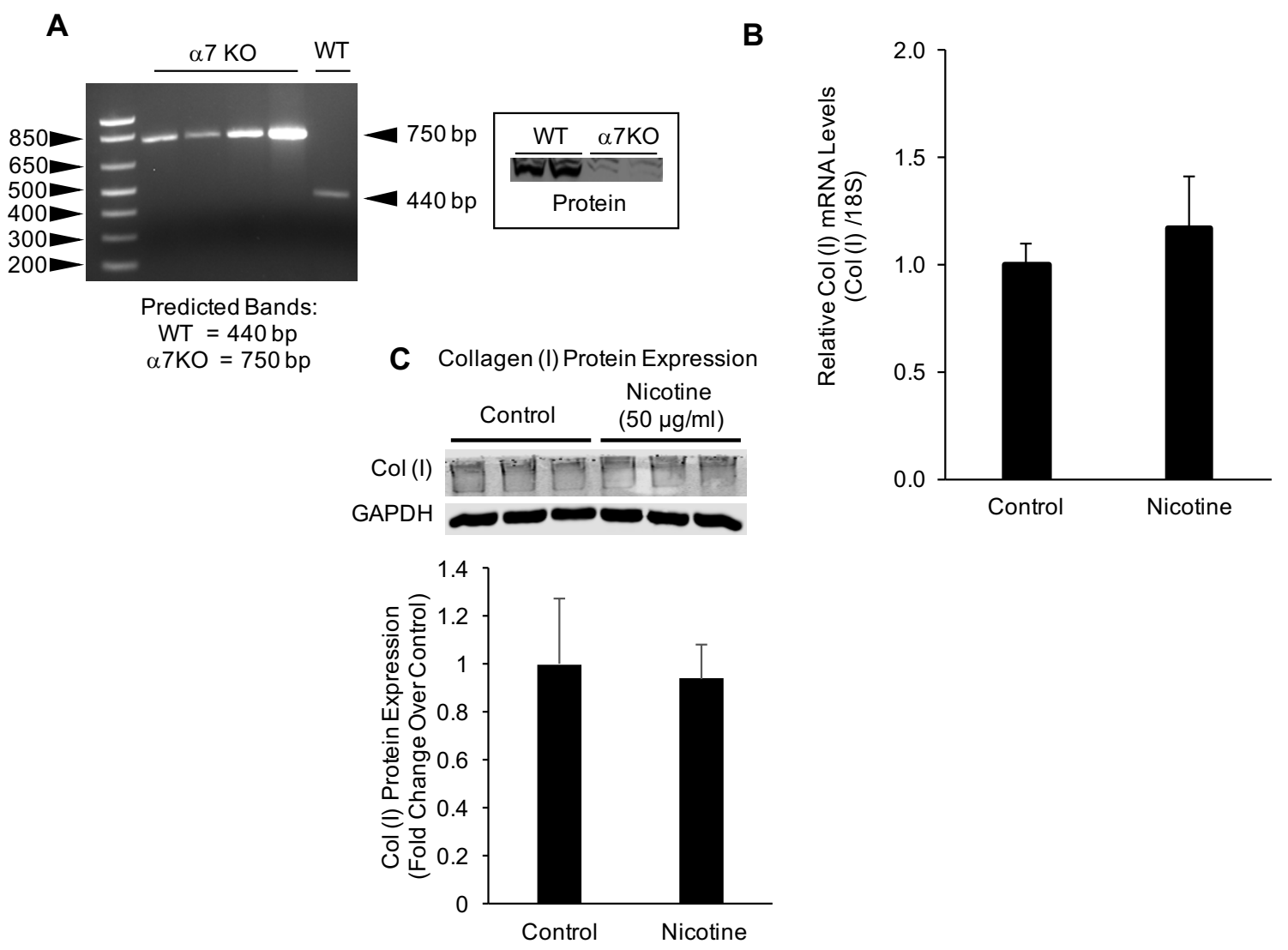

Figure 12. Nicotine Induces Collagen Type I Through $\alpha 7$ nAChRs

Vicary et. Al. 2016

(A) The absence of $\alpha 7 \mathrm{nAChR}$ was verified by mRNA and protein expression in the $\alpha 7 \mathrm{nAChR}$ deficient ( $\alpha 7 \mathrm{KO})$ mice. (B) $\alpha 7 \mathrm{KO}$ fibroblasts were exposed to nicotine for 24 hours. Nicotine failed to stimulate collagen type I mRNA expression in $\alpha 7 \mathrm{KO}$ cells. (C) Nicotine-treated $\alpha 7 \mathrm{KO}$ fibroblasts were subjected to Western blot analysis. Nicotine did not stimulate increased collagen deposition in $\alpha 7 \mathrm{KO}$ fibroblasts. Experiments were repeated at least 3 times. Significance was assessed using $p$ values $<0.05$ obtained by two-tailed t-test. 


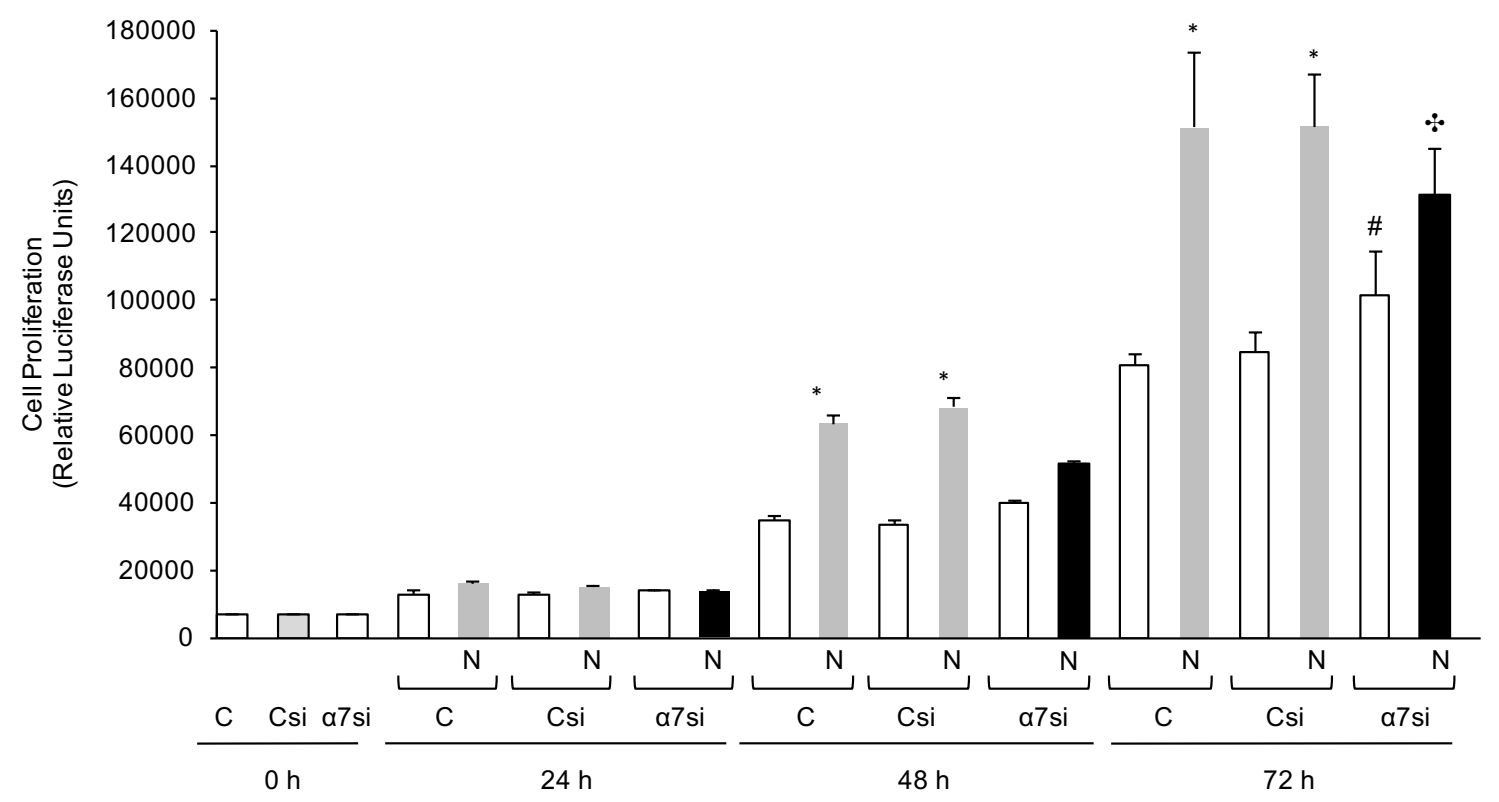

Figure 13. Nicotine Stimulates the Proliferation of Lung Fibroblasts via $\alpha 7$ nAChRs

Vicary et. Al. 2016

Lung fibroblasts were cultured for up to 72 hours after transfection with control (Csi) or $\alpha 7$ nAChR siRNAs ( $\alpha 7 \mathrm{si})$. At the appropriate times, the experiment was halted and live cells counted. Nicotine stimulated cell proliferation at 48 and 72 hours when compared to control cells $\left(^{*}\right)$. Absence of the a7 nAChR induced significant proliferation compared to control cells at 72 hours (\#). $\alpha 7 \mathrm{nAChR}$ siRNA inhibited the nicotine-induced response when compared to control cells at 48 hours and significantly decreased proliferation at 72 hours $(\because)$. Experiments were repeated at least 3 times. Significance was assessed using $p$ values $<0.05$ obtained by 2-way ANOVA with Bonferroni posttest. 


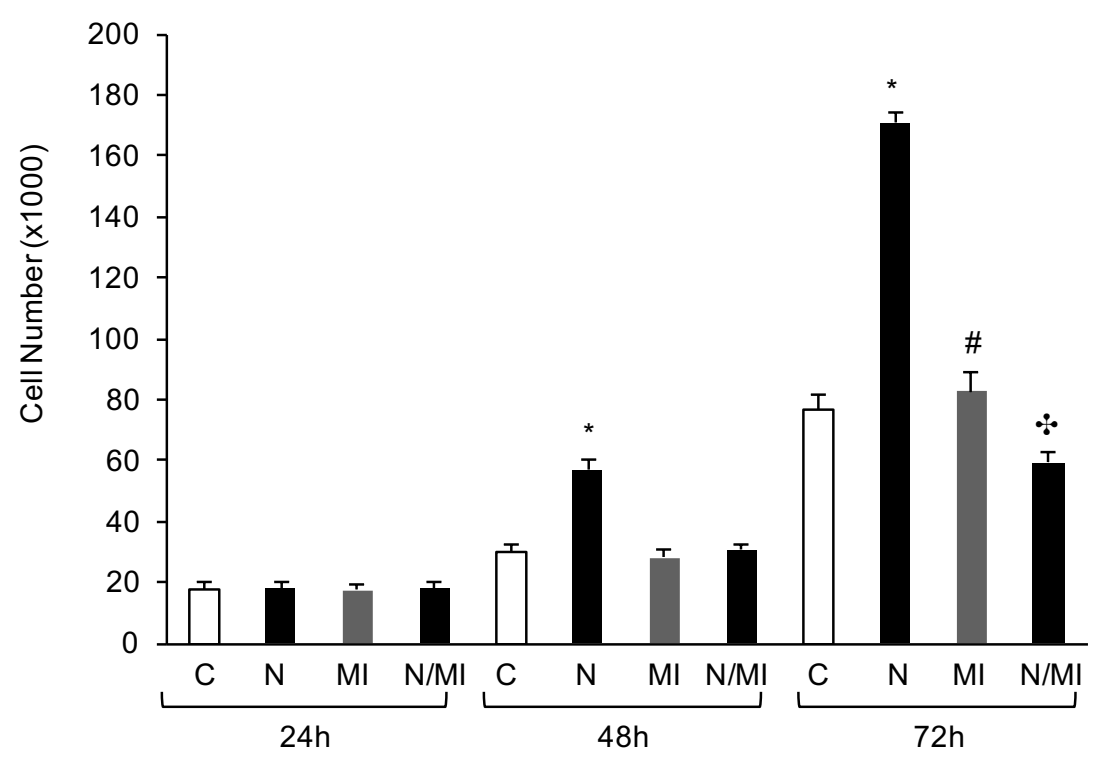

Figure 14. Nicotine Stimulates the Proliferation of Lung Fibroblasts via Induction of ERK

Fibroblasts were cultured for up to 72 hours with or without nicotine $(50 \mu \mathrm{g} / \mathrm{ml})$ in the presence or absence of PD98059 (MI, $50 \mu \mathrm{M})$, an inhibitor of MEK-1. Nicotine stimulated fibroblast proliferation at 48 hours, but the effect was most noticeable at 72 hours $\left(^{*}\right)$. The inhibitor PD98095 alone had a small effect on the proliferation of cells at 72 hours (\#), but did inhibited the stimulatory effect of nicotine $(\because)$. Experiments were repeated at least 3 times. Significance was assessed using $p$ values $<0.05$ obtained by 2 -way ANOVA with Bonferroni posttest. 


\section{Nicotine increases collagen expression in vivo}

To study the effects of nicotine in vivo, wildtype and $\alpha 7 \mathrm{KO}$ mice were exposed to nicotine in their drinking water $(100 \mu \mathrm{g} / \mathrm{ml})$ for 12 weeks to test if the mechanisms described above are relevant to the situation in vivo. This model has been shown to increase nicotine levels in blood and tissue similar to those of heavy smokers without affecting body weight [217]. Lower concentrations $(<1 \mu \mathrm{g} / \mathrm{ml})$ of nicotine can be found in the blood of smokers, while higher concentrations (1-10 $\mu \mathrm{g} / \mathrm{ml}$ ) are deposited in body tissues [216]. As depicted in Figure 15, the lungs of mice exposed to nicotine showed increased collagen type I mRNA expression. a7KO mice showed an insignificant increase in collagen type I mRNA. Protein analysis showed an increase in collagen type I, p-ERK 1/2, and p-Smad3 protein expression when compared to control lungs (Figure 16).

Histological analysis was performed on lung tissue harvested from untreated and nicotine-treated animals and found increased collagen deposition in wildtype mice treated with nicotine, noticeably around airway and vascular structures (red arrows) in tissues submitted to both Masson's tri-chrome and Sirius red staining (Figure 17). The increase in collagen was confirmed by blinded scoring of unlabeled tissue slides by eight investigators. Nicotine also failed to stimulate collagen deposition in the $\alpha 7 \mathrm{KO}$ mice (Figure 18). 


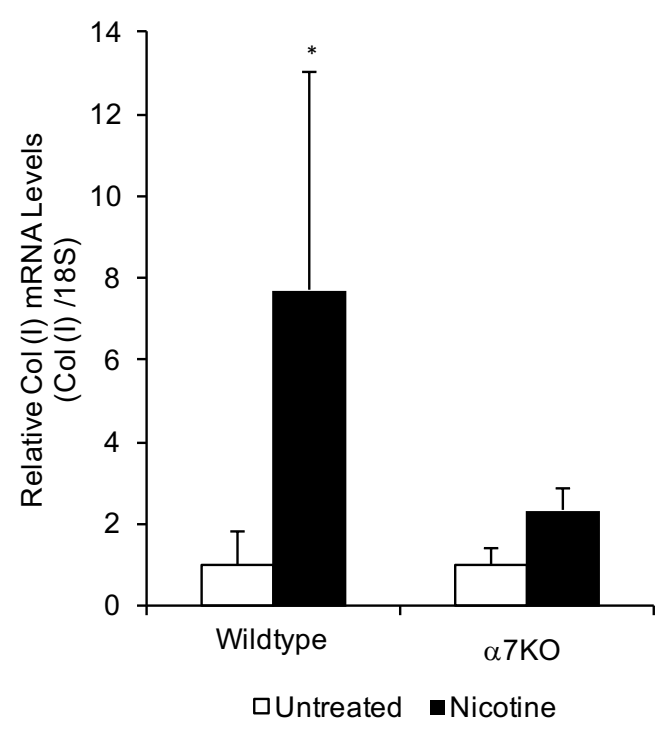

Figure 15. Nicotine Stimulates Collagen Type I mRNA Expression in Lung through the $\alpha 7$ nAChR In Vivo

Vicary et. Al. 2016

The lungs of wildtype mice exposed to nicotine $(100 \mu \mathrm{g} / \mathrm{ml})$ in the drinking water for 90 days were isolated for MRNA analysis, which showed an increase in collagen type I expression (Col (I)) in nicotine-treated wildtype mice, but not in $\alpha 7$ nAChR deficient ( $\alpha 7 \mathrm{KO})$ mice. Experiments were repeated at least 3 times. Significance was assessed using $p$ values $<0.05$ obtained by two-tailed t-test. 


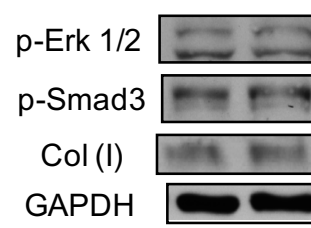

Control

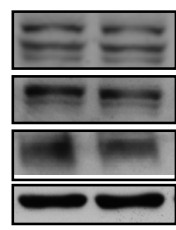

Nicotine

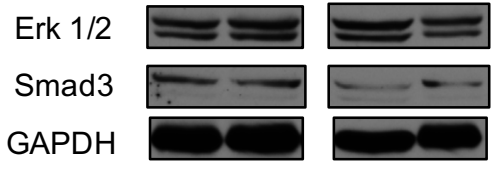

Control Nicotine

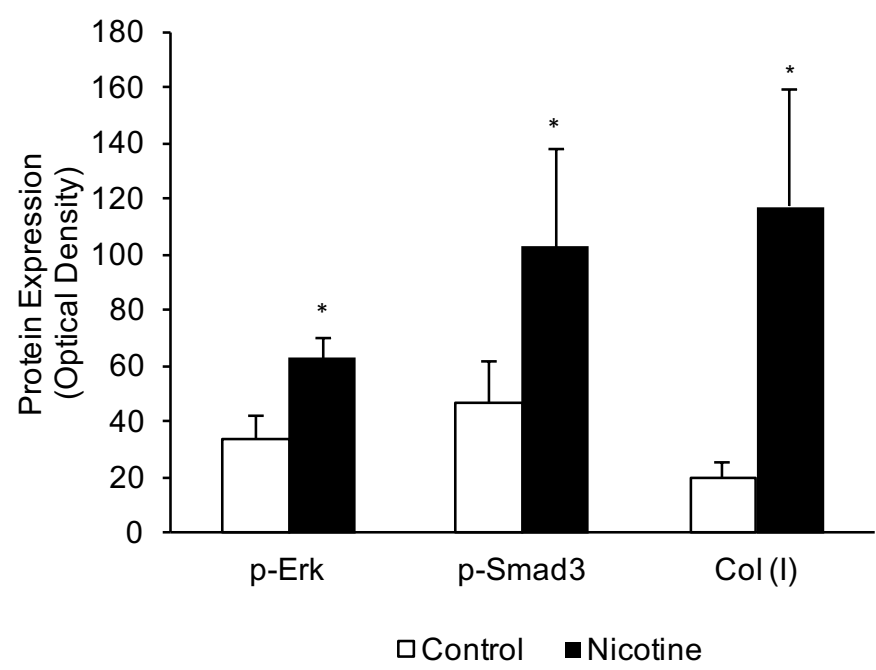

Figure 16. Nicotine Stimulates p-Erk, p-Smad3 and Collagen Type I Protein Expression in Lung through the $\alpha 7$ nAChR In Vivo

Vicary et. Al. 2016

Protein analysis the lungs of wildtype mice exposed to nicotine $(100 \mu \mathrm{g} / \mathrm{ml})$ in the drinking water for 90 days showed increased p-Smad3, p-Erk 1/2 and collagen type I $(\mathrm{Col}(\mathrm{I}))$ when compared to control lungs. P-Erk, p-Smad3, and collagen type 1 were all completed on the same gel, with the blot stripped between probings. Total Erk and Smad3 gels were run on a separate gel and stripped between probings. Experiments were repeated at least 3 times. Significance was assessed using $p$ values $<0.05$ obtained by two-tailed t-test. 
A

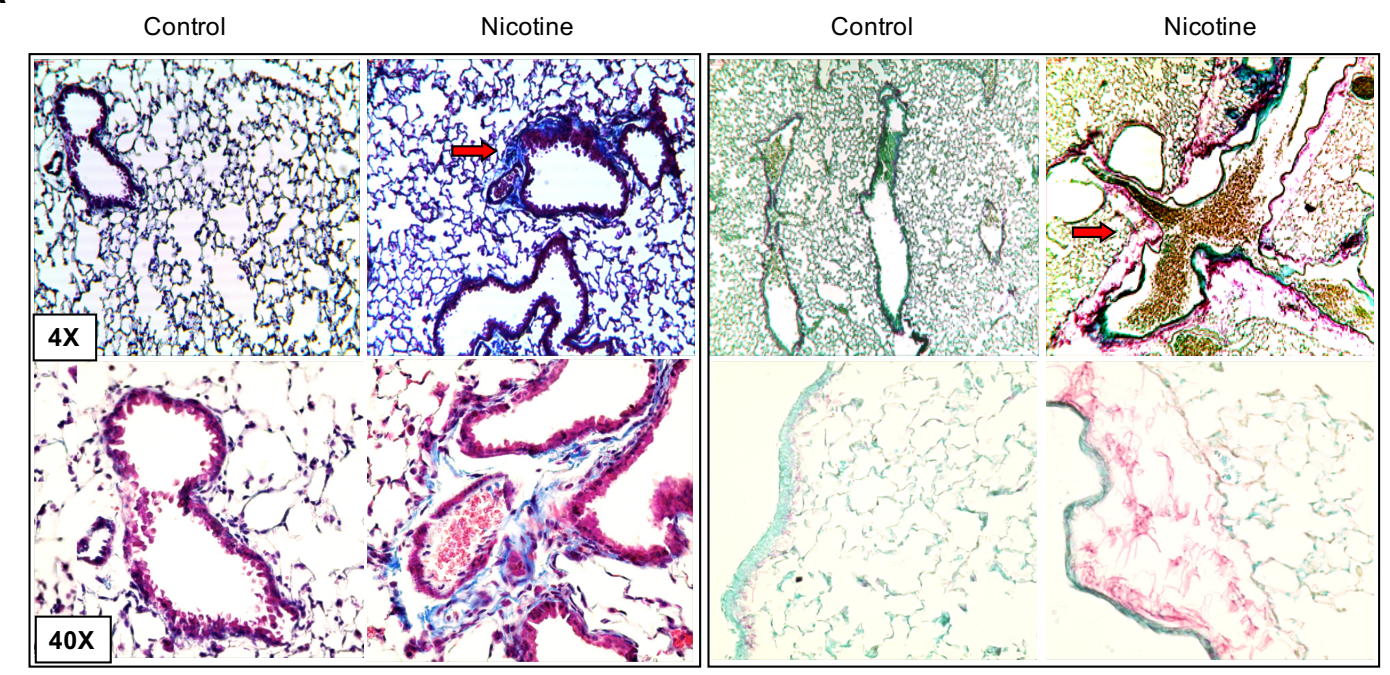

Masson's Tri-Chrome

Sirius Red/Fast Green

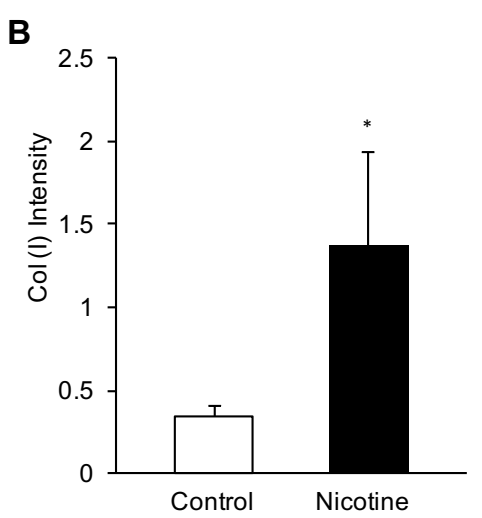

Figure 17. Nicotine Stimulates Collagen Expression in Lung In Vivo

Vicary et. Al. 2016

(A) The lungs of wildtype mice exposed to nicotine $(100 \mu \mathrm{g} / \mathrm{ml})$ in the drinking water for 90 days were inflated at standard pressure, fixed in formalin, paraffinembedded, and sectioned (5 $\mu \mathrm{m})$ for histological analysis. Lung sections were stained for tri-chrome or Sirius Red/Fast Green. Arrows indicate increased collagen type I deposition (blue stain or Red stain) in wildtype animals exposed to nicotine. (B) The tri-chrome slides were blindly graded on a scale of 0-3 based on the intensity of collagen staining. Significance was assessed using $p$ values $<0.05$ obtained by two-tailed t-test. 


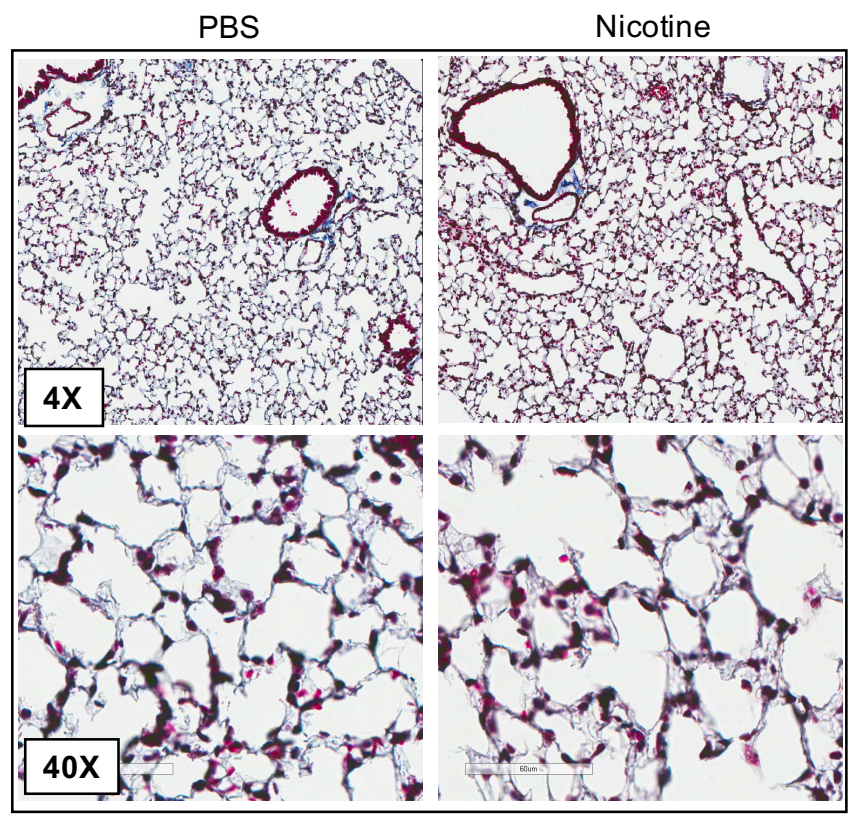

Figure 18. Nicotine-Induced Collagen Expression Mediated via $\alpha 7 \mathrm{nAChRs}$ in Lung In Vivo

Lungs of $\alpha 7 \mathrm{KO}$ mice exposed to nicotine $(100 \mu \mathrm{g} / \mathrm{ml})$ in the drinking water for 90 days were inflated at standard pressure, fixed in formalin, paraffin-embedded, and sectioned $(5 \mu \mathrm{m})$ for histological analysis. Nicotine treatment resulted in no differences in tri-chrome staining in $\alpha 7 \mathrm{nAChR}$ deficient mice. 


\section{Extracellular matrices derived from nicotine-treated lung fibroblasts stimulate monocytic cell expression of IL-1 $\beta$}

We then investigated whether matrices derived from nicotine-treated fibroblasts exert a differential effect on immune cells. For this, we cultured U937 human monocytic cells atop of matrices derived from untreated or nicotine-treated fibroblasts; U937 cells expressed the human IL-1 $1 \beta$ gene promoter fused to a luciferase reporter gene [214]. As presented in Figure 19, we found that IL-1® gene transcription was increased about 2-fold when cultured atop of matrices derived from nicotine-treated fibroblasts when compared to cells cultured atop matrices derived from untreated fibroblasts. This effect appeared to be mediated by collagen binding to $\alpha 2 \beta 1$, a collagen integrin receptor, since anti- $\alpha 2 \beta 1$ antibodies inhibited the induction of IL-1 $\beta$ (Figure 19). Importantly, IL-1 $\beta$ gene transcription was not increased in U937 cells cultured atop matrices derived from nicotine-treated $\alpha 7 \mathrm{KO}$ lung fibroblast (Figure 20). Treatment of fibroblasts with MEK-1 inhibitor PD98059 or an $\alpha 7$ nAChR inhibitor MG 624 prior to exposure to nicotine blunted U937 IL-1 $\beta$ induction by the matrix (Figure 21, Figure 22). Lastly, this process might be relevant in vivo since whole lung MRNA from nicotine-treated mice showed elevated IL-1 $\beta$ gene transcription and staining by immunohistochemistry (Figure 23). 


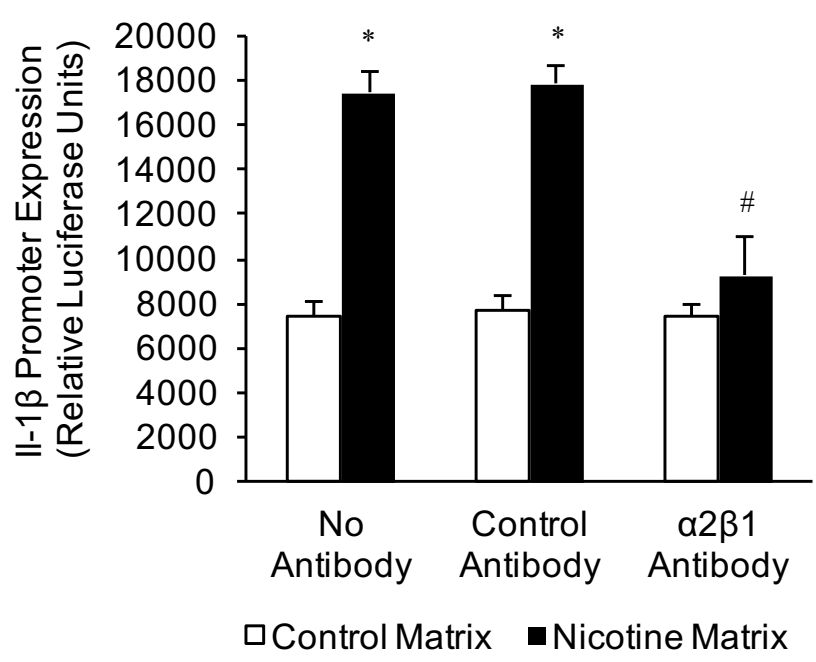

Figure 19. Matrices Derived from Nicotine-Treated Fibroblasts Stimulate IL$1 \beta$ Expression in Monocytic Cells Through the $\alpha 2 \beta 1$ Integrin Receptor

Vicary et. Al. 2016

Lung fibroblasts $\left(5 \times 10^{4}\right.$ cells $/ 12$ well) were treated with nicotine $(50 \mu \mathrm{g} / \mathrm{ml})$ for 120 hours. Fibroblasts were removed by osmotic lysis, the plates were washed thoroughly, and human monocytic U937 cells expressing the human interleukin-1 $\beta$ gene promoter connected to a luciferase reporter gene were overlaid atop the fibroblast-derived matrix. Afterwards, expression of the IL-1 $\beta$ promoter was analyzed by luciferase assay. Collagen-containing matrices derived from nicotinetreated fibroblasts stimulated monocytic cells to express the pro-inflammatory cytokine IL-1 $\beta\left(^{*}\right)$. Furthermore, nicotine-treated fibroblast matrix induction of IL-1 $\beta$ was significantly decreased by anti- $\alpha 2 \beta 1$ integrin antibodies (\#). Experiments were repeated at least 3 times. Significance was assessed using $p$ values $<0.05$ obtained by 2-way ANOVA with Bonferroni posttest. 


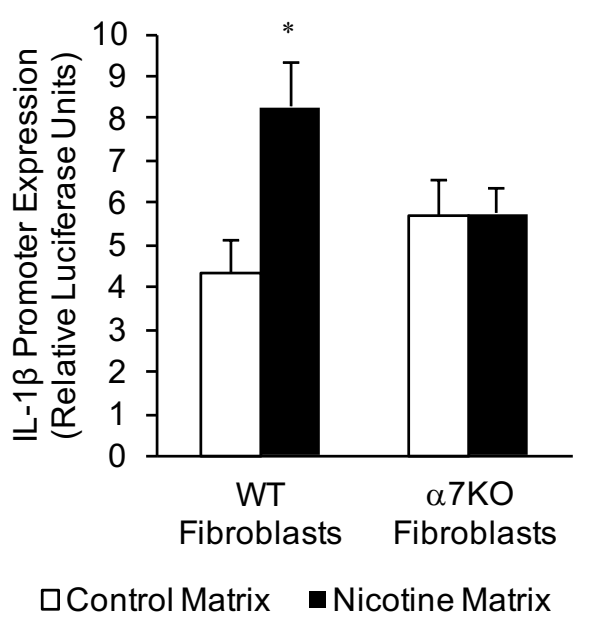

Figure 20. Pro-Inflammatory Matrices Derived from Nicotine-Treated Fibroblasts Mediated by $\alpha 7 \mathrm{nAChR}$

Vicary et. Al. 2016

Wildtype and $\alpha 7 \mathrm{nAChR}$ deficient lung fibroblasts ( $\alpha 7 \mathrm{KO})\left(5 \times 10^{4}\right.$ cells $/ 12$ well) were treated with nicotine $(50 \mu \mathrm{g} / \mathrm{ml})$ for 120 hours. Fibroblasts were removed by osmotic lysis, the plates were washed thoroughly, and human monocytic U937 cells expressing the human interleukin- $1 \beta$ gene promoter connected to a luciferase reporter gene were overlaid atop the fibroblast-derived matrix. Afterwards, expression of the IL-1 $\beta$ promoter was analyzed by luciferase assay. IL-1 $\beta$ gene transcription was not increased in U937 cells cultured on matrices derived from nicotine-treated $\alpha 7 \mathrm{nAChR}$ deficient primary lung fibroblast matrix over control. Experiments were repeated at least 3 times. Significance was assessed using $p$ values $<0.05$ obtained by 2 -way ANOVA with Bonferroni posttest. 


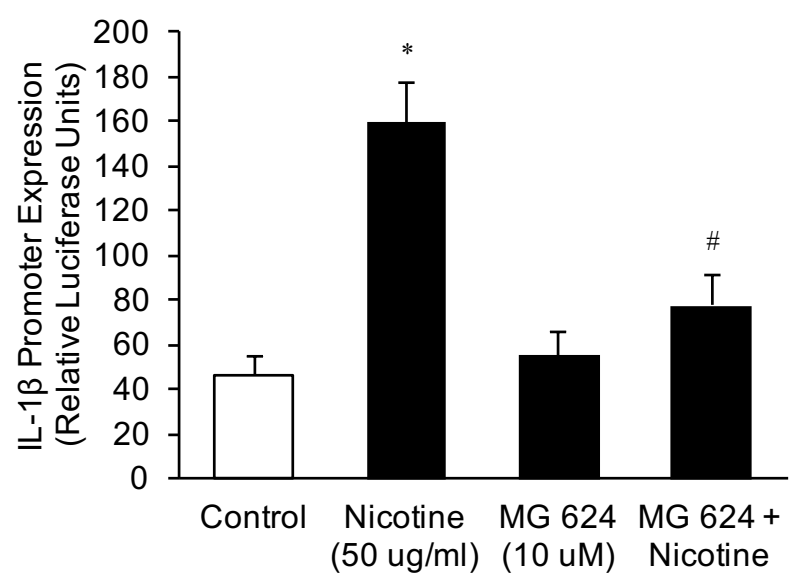

Figure 21. Pro-Inflammatory Matrices Derived from Nicotine-Treated Fibroblasts Blocked by $\alpha 7$ nAChR Inhibitor

Wildtype lung fibroblasts $\left(5 \times 10^{4}\right.$ cells $/ 12$ well) were treated with nicotine $(50 \mu \mathrm{g} / \mathrm{ml})$ and MG 624, an $\alpha 7 \mathrm{nAChR}$ antagonist $(10 \mu \mathrm{M})$, for 120 hours. Fibroblasts were removed by osmotic lysis, the plates were washed thoroughly, and human monocytic U937 cells expressing the human interleukin-1 $\beta$ gene promoter connected to a luciferase reporter gene were overlaid atop the fibroblast-derived matrix. Afterwards, expression of the IL-1 $\beta$ promoter was analyzed by luciferase assay. Fibroblasts treated with MG 624 concurrently with nicotine inhibited IL-1 $\beta$ expression without affecting baseline expression. Experiments were repeated at least 3 times. Significance was assessed using $p$ values $<0.05$ obtained by 2 -way ANOVA with Bonferroni posttest. 


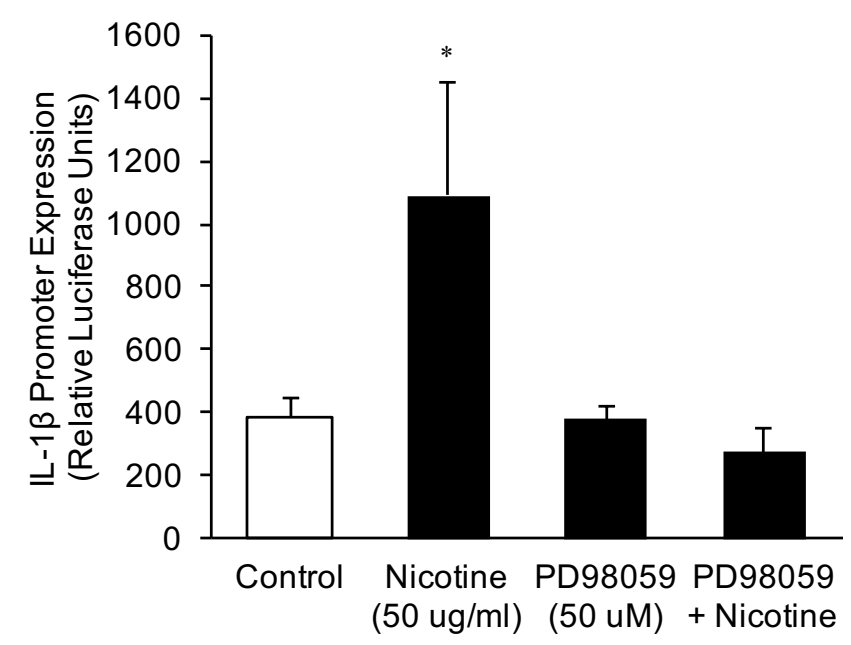

Figure 22. Pro-Inflammatory Matrices Derived from Nicotine-Treated Fibroblasts Blocked by MEK-1 Inhibitor

Vicary et. Al. 2016

Wildtype lung fibroblasts $\left(5 \times 10^{4}\right.$ cells $/ 12$ well $)$ were treated with nicotine $(50 \mu \mathrm{g} / \mathrm{ml})$ and PD98059, MEK-1 inhibitor (50 $\mu \mathrm{M})$, for 120 hours. Fibroblasts were removed by osmotic lysis, the plates were washed thoroughly, and human monocytic U937 cells expressing the human interleukin- $1 \beta$ gene promoter connected to a luciferase reporter gene were overlaid atop the fibroblast-derived matrix. Afterwards, expression of the IL-1 $\beta$ promoter was analyzed by luciferase assay. Fibroblasts treated with PD98059 concurrently with nicotine inhibited IL-1 $\beta$ expression without affecting baseline expression. Experiments were repeated at least 3 times. Significance was assessed using $p$ values $<0.05$ obtained by 2 -way ANOVA with Bonferroni posttest. 
A

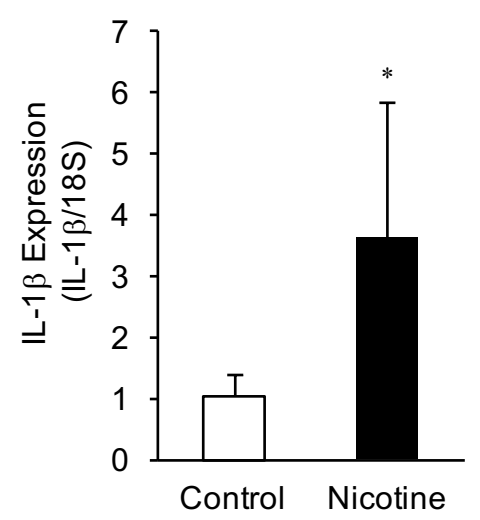

B

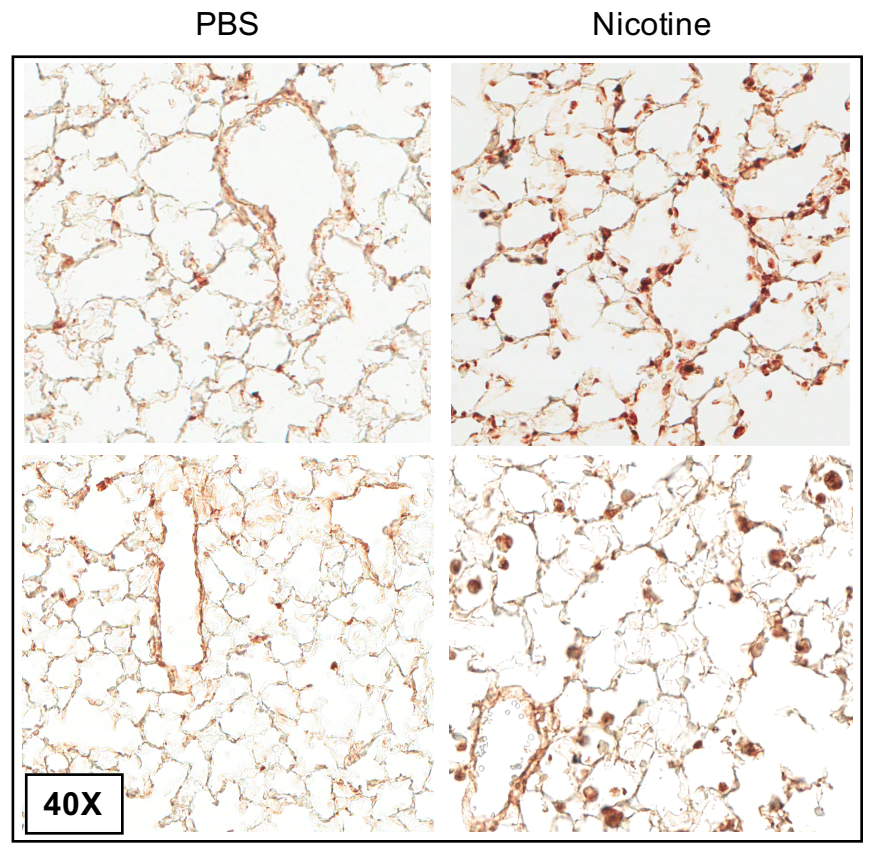

Figure 23. Nicotine Stimulates IL-1 $\beta$ Expression in Lung In Vivo

Vicary et. Al. 2016

(A) The lungs of mice exposed to nicotine $(100 \mu \mathrm{g} / \mathrm{ml})$ in the drinking water for 90 days were isolated for mRNA analysis, which showed an increase in IL-1 $\beta$ gene transcription by RT-PCR. (B) Lungs from control and nicotine-treated mice were stained by immunohistochemistry for IL-1 $\beta$. Increased staining was present in mice treated with nicotine. Experiments were repeated at least 3 times. Significance was assessed using $\mathrm{p}$ values $<0.05$ obtained by two-tailed t-test. 


\subsection{Discussion}

Tobacco-related lung disease is an important health problem worldwide. Several studies suggest that tobacco exposure promotes lung tissue remodeling through oxidant stress, inflammation, and the induction of matrix-degrading proteases, among other mechanisms. The latter is supported by animal studies showing that overexpression of matrix metalloproteinase (MMP)-1 promotes the development of emphysema in transgenic mice, while the lack of MMP-12 is protective $[219,230]$. Furthermore, alterations in MMPs and other proteases have been detected in humans with tobacco-related lung disease [231]. Unfortunately, this information has not yet translated into the development of effective therapeutic strategies. Here, we explore another mechanism of action, the induction of lung tissue remodeling through stimulation of extracellular matrix deposition. We hypothesized that nicotine, a major component of tobacco, is not only involved in tobacco addiction, but stimulates lung fibroblasts to release matrix components that affect the relative composition of the lung matrix. Consistent with this idea, we found that nicotine stimulates lung fibroblast expression and release of collagen type I up to 72 hours.

Previously, we reported that nicotine stimulates the expression in lung of fibronectin, a matrix glycoprotein implicated in injury and repair [77]. However, fibronectin matrices are often considered 'transitional' matrices whose deposition does not necessarily lead to irreversible changes in tissue architecture in the absence of other factors. The discovery that nicotine also stimulates the deposition 
of fibrillar collagens (this dissertation) is important because it suggests that the effects of nicotine on matrix composition may be more permanent. Collagen type I is highly expressed in injured lungs as demonstrated in acute lung injury, COPD, and chronic fibrotic lung disorders [232]. Additionally, collagen has been associated with extralobar pulmonary artery stiffening caused by chronic hypoxia [233], induction of epithelial-to-mesenchymal transition in non-small cell lung cancer cell lines [234], and stimulation of cell chemotaxis by its fragments [235].

Considering the importance of the proposed mechanisms of action, we turned our attention to the pathways involved in stimulation of collagen expression. We found that nicotine affected collagen expression in lung fibroblasts by acting on $\alpha 7$ nAChRs. Several studies have implicated $\alpha 7$ nAChRs in lung branching morphogenesis and in the pathogenesis of lung cancer [236, 237]. More recently, $\alpha 7$ nAChRs were found to mediate the effects of nicotine in developing lungs [35, 238]. Specifically, morphological airway abnormalities and airflow limitation were detected in the offspring of nicotine-treated wildtype animals, but not in animals lacking $\alpha 7$ nAChRs. Interestingly, and reminiscent to our work, collagen was found to be upregulated around the airways of animals exposed to nicotine. Based on the information presented here and the growing number of publications implicating $\alpha 7 \mathrm{nAChRs}$ in multiple disease states, it is reasonable to consider $\alpha 7 \mathrm{nAChRs}$ as promising targets for drug development to counteract the deleterious effects of tobacco. This is now possible considering that the technology to develop safe and effective agents that target nAChRs are currently available for human use [239]. 
Another important finding was that nicotine also stimulated fibroblast proliferation, a process capable of further promoting tissue remodeling. This effect was also mediated via $\alpha 7 \mathrm{nAChRs}$ as demonstrated by the lack of response in cells silenced for $\alpha 7$ nAChRs. In prior work, we and others demonstrated that nicotine leads to ERK activation [77]. Consequently, we tested the role of ERK and found that a MEK-1/ERK inhibitor, PD98059, inhibited nicotine-induced fibroblast proliferation.

To determine the potential relevance of our in vitro findings, we tested nicotine exposure in vivo. Previous studies have shown nicotine increases collagen type I expression in vivo in rhesus monkeys [90]. We exposed mice to nicotine in their drinking water for 8 weeks. When examined, the harvested lungs from nicotine-treated wildtype mice showed increased collagen deposition predominating around airway and vascular structures as determined by immunohistochemistry. Consistent with our in vitro findings, lung tissue also showed increased phosphorylation of ERK. We also detected increased phosphorylation of Smad3, a transcription factor known for mediating many of the pro-fibrotic effects of transforming growth factor- $\beta$. Increased Smad3 has been associated with collagen expression in cardiac and dermal fibroblasts [240, 241].

Our findings suggest that nicotine is capable of promoting fibronectin [77] and collagen type I (this report) deposition in lung without affecting the organ's overall architecture. We refer to this process, which appears to be self-limiting, as "transitional remodeling". Since deposition of new collagen fibrils in our model were not associated with dramatic alterations in lung architecture, how then do these 
subtle changes in lung matrix composition affect the lung? We reasoned that these newly deposited collagen fibrils do not affect the lung in the absence of other injurious stimuli, but instead, may influence immune cell function after injury. Consistent with this idea, our lab has previously shown that purified collagen type I can robustly activate monocytic IL-1 $\beta$ expression [242]. In this report, we show that collagen-containing matrices derived from nicotine-treated fibroblasts are capable of activating monocytic cells and stimulating their expression of the proinflammatory cytokine IL-1 $\beta$. This pro-inflammatory event was inhibited by pretreating fibroblasts with antibodies against $\alpha 2 \beta 1$, a collagen-binding integrin [243], PD98059 (MEK-1 inhibitor), MG 624 ( $\alpha 7$ nAChR inhibitor), or matrices derived from nicotine-treated $\alpha 7 \mathrm{KO}$ primary lung fibroblasts when compared to control. Increased IL-1 $\beta$ gene expression was also detected in the lungs of nicotine-treated mice (Figure 23). In addition to monocytic cells, integrins also control immune responses in T cells. For example, integrin-mediated binding to collagen provides a co-stimulatory signal for T cell activation [244], resulting in increased proliferation and secretion of pro-inflammatory cytokines such as TNF$\alpha$ and IFN- $\gamma$ [245]. Thus, by promoting subtle alterations in matrix composition, nicotine may indirectly stimulate the exaggerated expression of pro-inflammatory cytokines (e.g., IL-1 $\beta$ ) by immune cells recruited to the lung after injury, thereby helping perpetuate inflammation, a process considered important in the pathogenesis of tobacco-related lung disorders.

Elements of lung transitional remodeling have also been demonstrated in alcoholexposed rats and mice [211, 246], alcoholic subjects [212], post-lung transplant 
recipients [247], and aging mice [70]. However, the implications of lung transitional remodeling are unknown. It is presumed that if the stimulating agent is eliminated, a 'normal' matrix is restored. In contrast, persistence of the transitional matrix may lead to ineffective repair after injury through the induction of pro-inflammatory agents directly or via the release of matrix fragments [248]. We and others have suggested that these changes may explain the increased incidence and mortality observed for acute lung injury in alcoholics $[212,213]$, the predisposition to lung cancer in smokers [92, 249], the development of rejection after lung transplantation [247], and the worse outcomes observed in elderly patients with pulmonary disorders [70].

Additionally, it is important that we emphasize the implications of this research to understanding the potential impact (and safety) of e-cigarettes, an area that remains relatively unexplored. Consistent with this report, early studies suggested that e-cigarettes cause similar cell changes as those caused by tobacco exposure [250]. Considering this, in addition to serious concerns about their ability to serve as a gateway drug for smoking and current non-smokers, the use of these agents might pose a serious setback for global health with the use of e-cigarettes in adolescents, which has doubled yearly [251, 252].

However, until further studies are performed, these statements remain highly speculative. Booth et al., recently published a technique to isolate acellular lungs, which could provide the methods to study this interaction in a transitional matrix lung [59]. Nevertheless, the idea that lung transitional remodeling may 
precede processes such as COPD, acute lung injury and pulmonary fibrosis, among other disorders, is tantalizing and testable.

In summary, chronic nicotine exposure in mice results in lung matrix remodeling characterized by increased collagen type I expression/deposition in lung as well as phosphorylation of ERKs and Smad3. In the absence of injury, this subtle change in matrix composition does not affect the overall lung architecture, but may promote exaggerated inflammatory (e.g., induction of IL-1 $\beta$ by immune cells) and repair (e.g., fibroproliferation) responses after injury. These events appear to be mediated via $\alpha 7 \mathrm{nAChRs,} \mathrm{which} \mathrm{may} \mathrm{represent} \mathrm{promising} \mathrm{targets} \mathrm{for}$ intervention if lung transitional remodeling is proven to be a pre-disease susceptibility state that precedes (and promotes) lung destruction after injury. 


\section{CHAPTER IV}

\section{THE EFFECTS OF CHRONIC NICOTINE EXPOSURE ON INJURY-INDUCED FIBROSIS}

\subsection{Introduction}

Smoking is considered the most important contributing factor in the development of fibrosing lung disorders including idiopathic pulmonary fibrosis, where over half of patients with the disease are smokers or ex-smokers [23, 25]. IPF is characterized by progressive lung tissue remodeling and affects over 100,000 Americans [1].

How smoking contributes to tissue remodeling is unclear and work in this area has been hampered by the complex nature of tobacco smoke, which contains over 7,000 compounds $[26,27]$. Recently, attention has turned to the potential role of nicotine, an alkaloid present in tobacco, known for driving the addictiveness of cigarettes. Nicotine has been shown to affect several biological processes including cell apoptosis, migration, invasion, angiogenesis, inflammation and immunity [33]. Additionally, the Roman lab has previously demonstrated that 
nicotine stimulates the proliferation of primary lung fibroblasts as well as their production of fibronectin, a matrix glycoprotein implicated in tissue injury and disrepair $[46,77]$. I have also found that nicotine promotes the expression and deposition in lung of collagen type I, a fibrillar collagen highly expressed in maladaptive repair (Chapter 3). Thus, it appears that nicotine is not just responsible for tobacco dependence, but might also contribute to the pathogenesis of chronic lung disease. However, the exact mechanisms by which nicotine contributes to the pathophysiology of pulmonary fibrosis is unknown. Thus, we set out to investigate this in a murine model of lung fibrosis.

Several models have been used to study pulmonary fibrosis, yet none of these models have accurately reproduced the human condition at this time. Bleomycin-induced lung fibrosis is the most commonly used model use to investigate the mechanisms that lead to tissue remodeling after lung injury [160]. Studies performed in the bleomycin model of lung injury have provided some insight into the role of cigarette smoke. Guinea pigs exposed to cigarette extract for example, having worse lung fibrosis than the bleomycin treatment alone group [175]. Similarly, bleomycin-induced alveolar architecture derangement was enhanced by cigarette smoke in hamsters [173]. In another study, however, cigarette smoke exposure failed to enhance bleomycin-induced lung fibrosis in rats, but cigarette smoke exposure was not initiated till post bleomycin instillation [176]. Thus, there appears to be a role for tobacco exposure in pulmonary fibrosis, but much remains to be elucidated. To date, no studies have been published on the role of nicotine in bleomycin-induced lung fibrosis. We hypothesized that 
increased baseline levels of fibronectin and collagen due to nicotine exposure, creating a transitional matrix, would increase bleomycin-induced lung fibrosis.

Nicotine induced fibronectin and $\alpha$-SMA expression in pulmonary fibroblasts and mice. The nicotine induction was ablated in $\alpha 7 \mathrm{nAChR}$ deficient fibroblasts and mice. Additionally, nicotine induced ECM production indirectly, with bronchoalveolar lavage fluid from nicotine-treated mice increasing fibronectin expression in fibroblasts.

To investigate the role of this transitional matrix in pulmonary fibrosis, wildtype and $\alpha 7$ deficient mice ( $\alpha 7 \mathrm{KO}$ ) were treated with nicotine for 90 days, then instilled with bleomycin and euthanized 21 days later. Mice treated with nicotine and bleomycin had significantly more fibrosis when compared to bleomycin alone, but the role of $\alpha 7 \mathrm{nAChRs}$ in mediating this effect was less clear, with $\alpha 7 \mathrm{KO}$ mice having increased fibrosis at baseline when compared to wildtype, but nicotine exposure lowered fibrosis in $\alpha 7 K O$ mice to wildtype levels. Additionally, I investigated the mechanobiology effects of nicotine-induced fibrosis on the lung, but no significant differences in lung stiffness were detected between untreated and nicotine-treated mice instilled with bleomycin. In conclusion, subtle changes induced by nicotine may render the host susceptible to excessive tissue damage after injury. 


\section{$\underline{4.2 \text { Results }}$}

\section{Nicotine stimulates fibronectin and $\alpha$-SMA expression in vitro and in vivo via}

$\alpha 7$ nAChRs

Primary lung fibroblasts from wildtype and $\alpha 7 \mathrm{KO}$ mice were cultured with nicotine for 24 hours and isolated for protein and mRNA analysis. As depicted in Figure 24 and Figure 25, nicotine stimulation enhanced fibronectin and $\alpha$-SMA expression in wildtype lung fibroblasts, but was absent in $\alpha 7 \mathrm{KO}$ cells. In Vivo, nicotine stimulated fibronectin and $\alpha$-SMA expression in the lungs of wildtype mice, but not in $\alpha 7 \mathrm{KO}$ mice (Figure 26 and Figure 27). Similarly, transgenic mice expressing the human fibronectin gene fused to a luciferase reporter gene were exposed to nicotine in their drinking water for up to 90 days. Increased expression of luciferase (i.e. fibronectin) throughout the lung indicates the induction of fibronectin deposition in lung is due to increased expression in several resident lung cell types (Figure 28). Furthermore, the ability of the bronchoalveolar lavage fluid of nicotine-treated animals to stimulate fibronectin expression was investigated. As depicted in Figure 29, BAL fluid harvested from nicotine-treated animals stimulates fibronectin expression in otherwise untreated NIH3T3 lung fibroblasts. We found that this effect appears specific since the BAL fluid of nicotine-treated animals did not stimulate THP monocytes to express MMP-9, an enzyme involved in ECM breakdown and remodeling (Figure 29). 


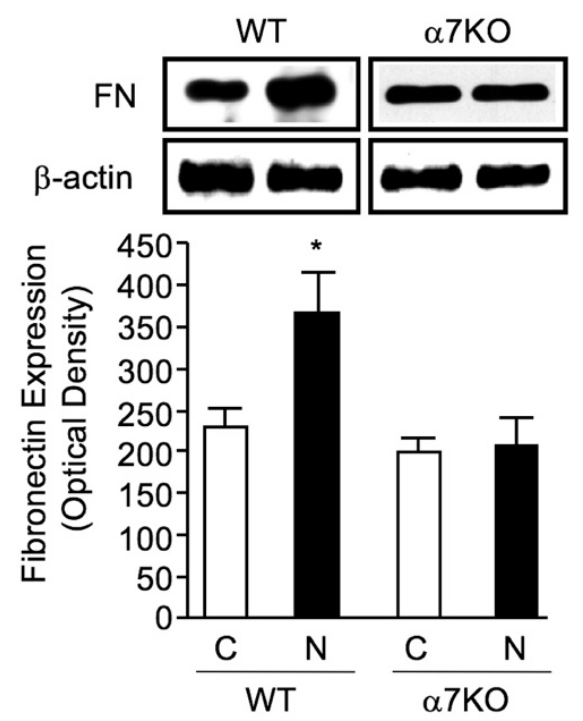

Figure 24. Nicotine Stimulates Fibronectin Protein Expression Through $\alpha 7$ nAChRs

Vicary et. Al., Unpublished

Fibroblasts were harvested from the lungs of wildtype (WT) and $\alpha 7 \mathrm{nAChR}$ deficient mice $(\alpha 7 \mathrm{KO})$ and cultured in the presence or absence of nicotine $(\mathrm{N})(50$ $\mu \mathrm{g} / \mathrm{ml}$ ) for 48 hours and isolated for western blot analysis. Actin was used for normalization purposes. Results expressed as mean optical density (OD) units. Nicotine stimulated fibronectin production in wildtype cells, but failed to induce a significant amount of fibronectin in the $\alpha 7 \mathrm{KO}$ fibroblasts. Experiments were repeated at least 3 times. Significance was assessed using $p$ values $<0.05$ obtained by two-tailed t-test. 


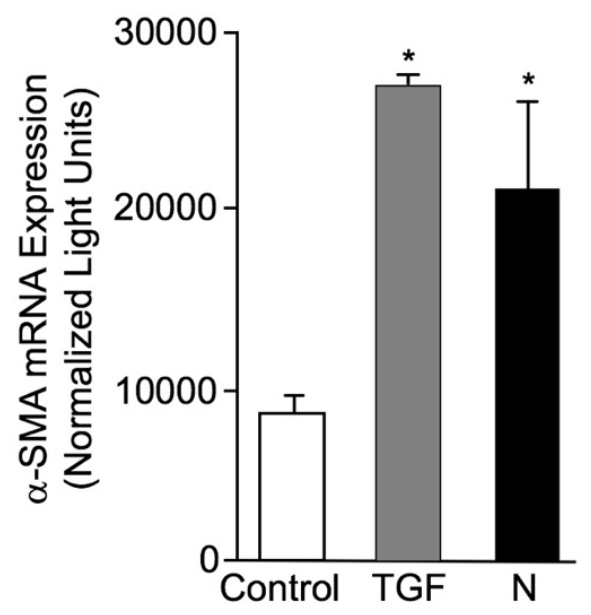

Figure 25. Nicotine Stimulates $\alpha$-Smooth Muscle Actin ( $\alpha$-SMA) mRNA Expression

Vicary et. Al., Unpublished

Fibroblasts were harvested from the lungs of wildtype mice and cultured in the presence or absence of nicotine $(\mathrm{N})(50 \mu \mathrm{g} / \mathrm{ml})$ for 48 hours and isolated for mRNA analysis. Actin was used for normalization purposes. TGF- $\beta 1$ (TGF) served as a positive control. Nicotine stimulated $\alpha$-SMA production in wildtype fibroblasts. Experiments were repeated at least 3 times. Significance was assessed using $p$ values $<0.05$ obtained by two-tailed t-test. 


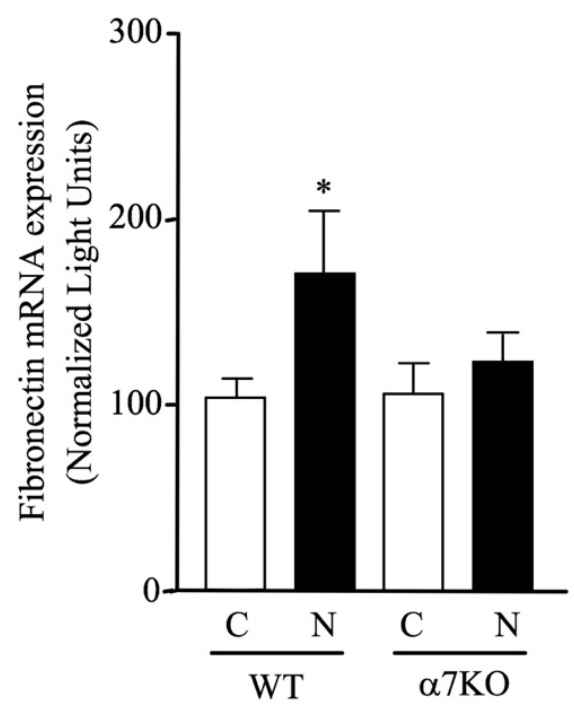

Figure 26. Nicotine Stimulates mRNA Fibronectin Expression through $\alpha 7$ nAChRs In Vivo

Wildtype (WT) and $\alpha 7 \mathrm{nAChR}$ deficient $(\alpha 7 \mathrm{KO})$ mice were exposed to nicotine $(100 \mu \mathrm{g} / \mathrm{ml})$ in the drinking water for 90 days. Lungs were isolated for mRNA analysis. $\beta$-actin was used for normalization purposes. Nicotine stimulated fibronectin production in wildtype lungs, but not in $\alpha 7 \mathrm{KO}$ mice. Experiments were repeated at least 3 times. Significance was assessed using $p$ values $<0.05$ obtained by two-tailed t-test. 


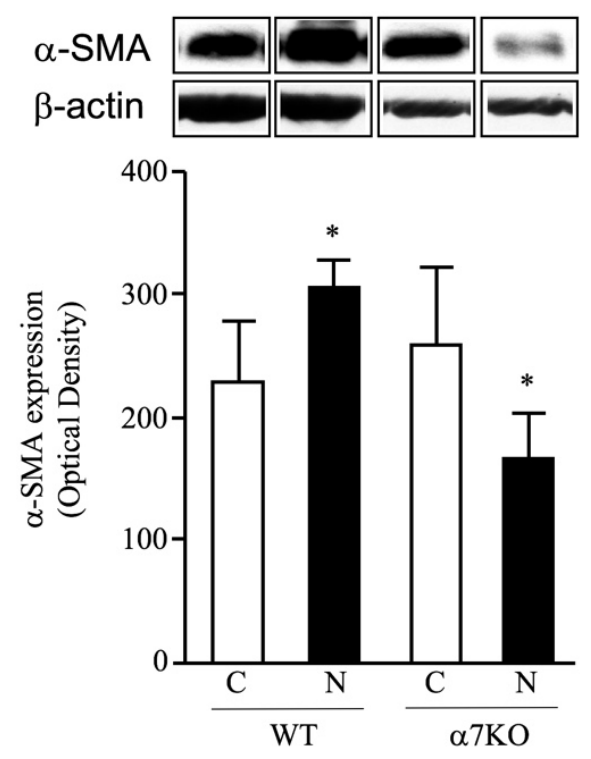

Figure 27. Nicotine Stimulates $\alpha$-SMA Protein Expression through $\alpha 7$ nAChRs In Vivo

Transgenic mice expressing the human fibronectin gene fused to a luciferase reporter gene were exposed to nicotine $(100 \mu \mathrm{g} / \mathrm{ml})$ in the drinking water for 90 days. Lungs were isolated for protein analysis. $\beta$-actin was used for normalization purposes. Results expressed as mean optical density (OD) units. Nicotine stimulated $\alpha$-SMA production in wildtype lungs, but not in $\alpha 7 \mathrm{KO}$ mice. Experiments were repeated at least 3 times. Significance was assessed using $p$ values $<0.05$ obtained by two-tailed t-test. 

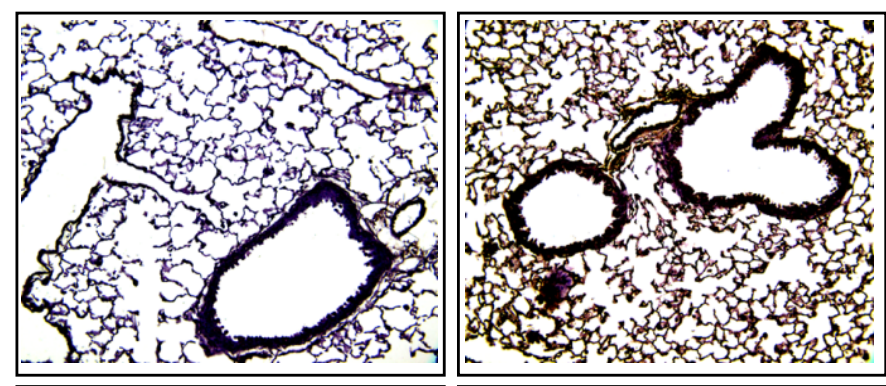

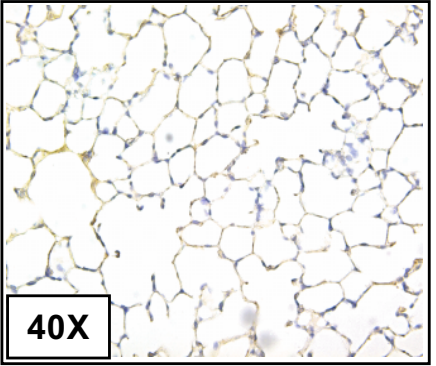

Control

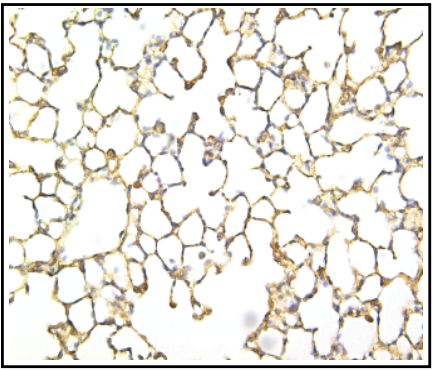

Nicotine

Figure 28. Nicotine Stimulates Fibronectin Expression through $\alpha 7 \mathrm{nAChRs}$ In Vivo

Transgenic mice expressing the human fibronectin gene fused to a luciferase reporter gene were exposed to nicotine $(100 \mu \mathrm{g} / \mathrm{ml})$ in the drinking water for 90 days. Lungs were isolated for histology. Increased expression of luciferase (connected to fibronectin gene) was detected throughout the lung indicating that the previously reported induction of fibronectin protein deposition in lung is due to increased gene expression in several resident lung cell types. Experiments were repeated at least 3 times. 
A

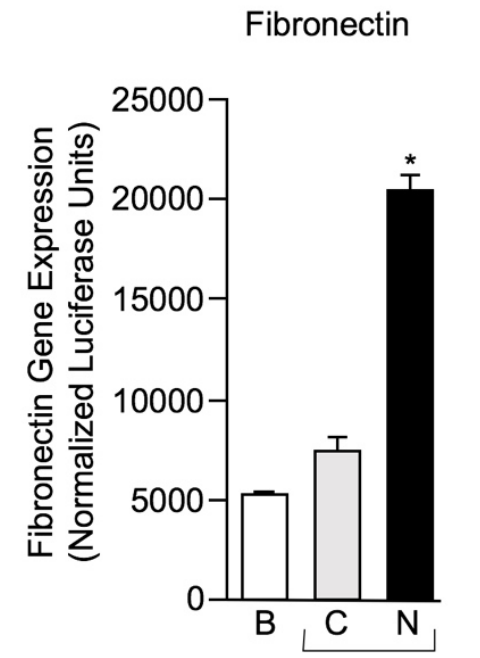

BALF treatment
B

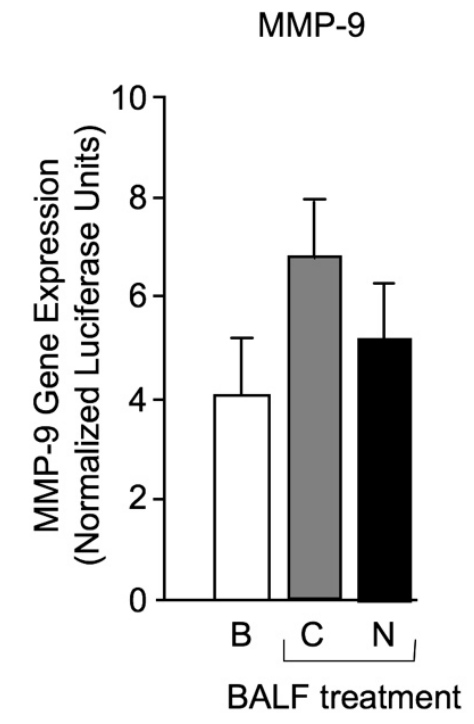

Figure 29. Bronchoalveolar Lavage (BAL) Fluid from Nicotine-Treated Mice Induced Fibronectin Expression in NIH3T3 Fibroblasts

Vicary et. Al., Unpublished

(A) BAL fluid was harvested from wildtype mice exposed to nicotine $(100 \mu \mathrm{g} / \mathrm{ml})$ in the drinking water for 90 days. BAL fluid was cultured with NIH3T3 fibroblasts transfected with the human fibronectin promoter connected to a luciferase reporter gene for 24 hours. BAL fluid from nicotine-treated mice induced significant expression of fibronectin. (B) BAL fluid did not stimulate the expression of the MMP-9 promoter in THP-1 cells transfected with a MMP-9 promoter-luciferase construct. B: baseline; C: BAL fluid from control animals; N: BAL fluid from nicotine treated animals. Experiments were repeated at least 3 times. Significance was assessed using $p$ values $<0.05$ obtained by two-tailed t-test. 


\section{Nicotine enhances bleomycin-induced lung fibrosis through $\alpha 7 \mathrm{nAChRs}$}

Having examined the effects of nicotine on fibronectin and $\alpha$-SMA expression in vitro and in vivo, we turned our attention to its effects on bleomycininduced lung fibrosis. Untreated and nicotine-treated animals were administered bleomycin $(1.0 \mathrm{U} / \mathrm{kg})$ intratracheally to promote the development of fibrosis. As hypothesized, bleomycin-induced lung fibrosis was increased in nicotine treated mice as observed by histology (Figure 30). However, the role of $\alpha 7 \mathrm{nAChRs}$ in mediating the increased fibrosis seen in nicotine-treated mice is unclear. $\alpha 7 \mathrm{KO}$ mice treated with bleomycin alone had increased fibrosis at when compared to wildtype mice treated with bleomycin alone, but was dissipated with nicotinetreatment (Figure 30).

\section{Creation of an ex vivo model and determination of lung stiffness}

Lungs from wildtype control and nicotine-treated mice instilled with bleomycin mice were decellularized by perfusions of $\mathrm{DI}$ solution $\left(\mathrm{dH}_{2} \mathrm{O}\right.$ with $1 \%$ Antibiotic Antimitotic Solution), 0.1\% Trition X-100, 2\% Na deoxycholate, 1M $\mathrm{NaCl}$, and DNAse solution and embedded in 6\% Seaprep Ultra Low Agarose (Figure 31). Acellular lung sections were cut in a vibratome and stained for hematoxylin, tri-chrome, and immunostaining. Lack of DAPI staining showed removal of cellular material with ECM remaining in normal architecture (Figure 32). 
Acellular lungs from bleomycin and bleomycin and nicotine treated mice were tested for possible mechanical property differences by atomic force microscopy. A force map of each sample was conducted and stiffness measurements (pascals) were averaged. There were no significant differences in lung stiffness between bleomycin and bleomycin and nicotine treated mice due to the large variation in stiffness within each lung (Figure 33). 
A

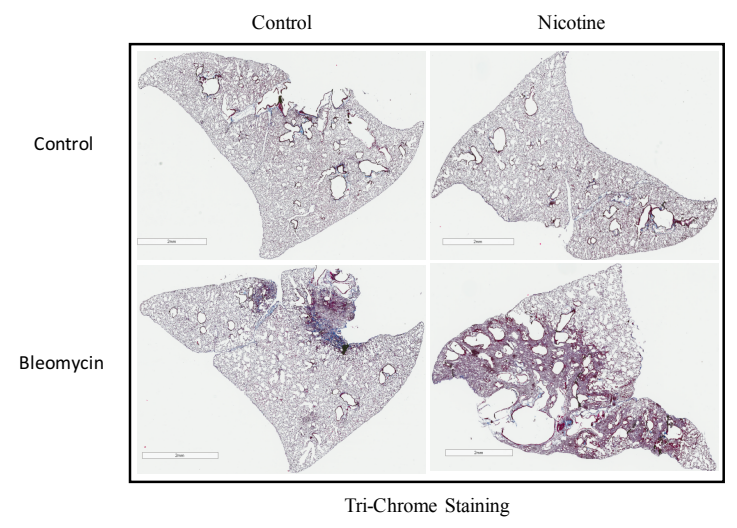

B

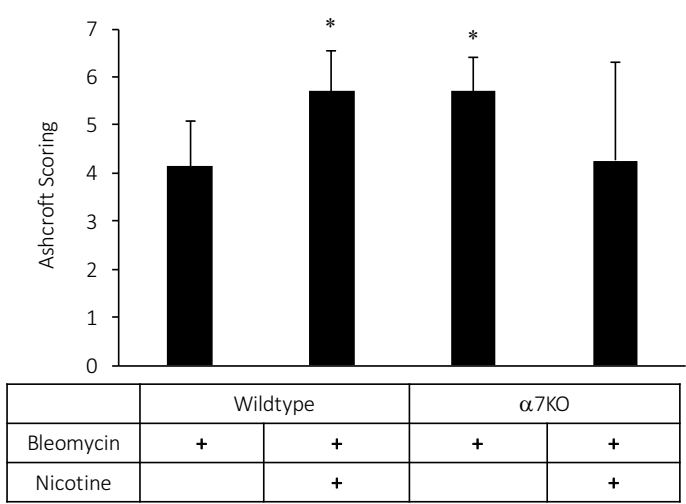

Figure 30. Nicotine Induces Bleomycin-Induced Fibrosis

Vicary et. Al., Unpublished

The lungs of wildtype and $\alpha 7 \mathrm{nAChR}$ deficient ( $\alpha 7 \mathrm{KO}$ ) mice exposed to nicotine $(100 \mu \mathrm{g} / \mathrm{ml})$ in the drinking water for 120 days. Bleomycin was administered intratracheally at a dose of $1.0 \mathrm{U} / \mathrm{kg}$ body weight. Surviving animals were sacrificed at 21 days post-bleomycin. (A) The lungs were processed and stained by Masson's trichrome staining. (B) Slides were blindly graded with PBS control lungs scored as 1 on the Ashcroft scale. Nicotine caused an increase in bleomycininduced pulmonary fibrosis at 21 days in WT mice. Nicotine's affects were not present in $\alpha 7 \mathrm{KO}$ mice, but interestingly, $\alpha 7 \mathrm{KO}$ mice had increased fibrosis with bleomycin alone when compared to wildtype mice. Experiments were repeated at least 3 times. Significance was assessed using $p$ values $<0.05$ obtained by twotailed t-test. 
A

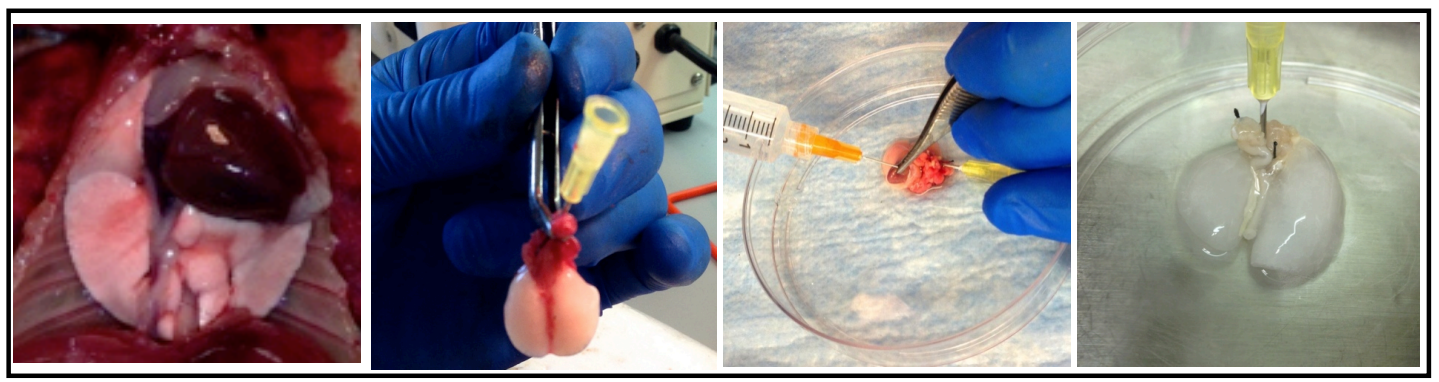

B

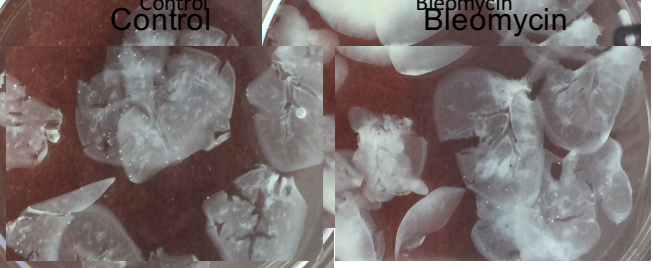

Figure 31. Lung Decellularization

Vicary et. Al., Unpublished

(A) Lungs were decellularized by perfusing $3 \mathrm{cc}$ of $\mathrm{H}_{2} \mathrm{O}$ solution into the right ventricle of the heart and trachea. Perfusions of Triton X-100, deoxycholate, $\mathrm{NaCl}$, DNase, and peracetic acid followed with incubations in each solution performed at $4^{\circ} \mathrm{C}$ on a rocker. Acellular lungs are embedded into low temperature SeaPrep ${ }^{\mathrm{TM}}$ agarose and cut using a vibratome. (B) Acellular lung sections from control and bleomycin-treated mice. Bleomycin-treated sections showed increased density of whiteness (i.e. ECM proteins). 


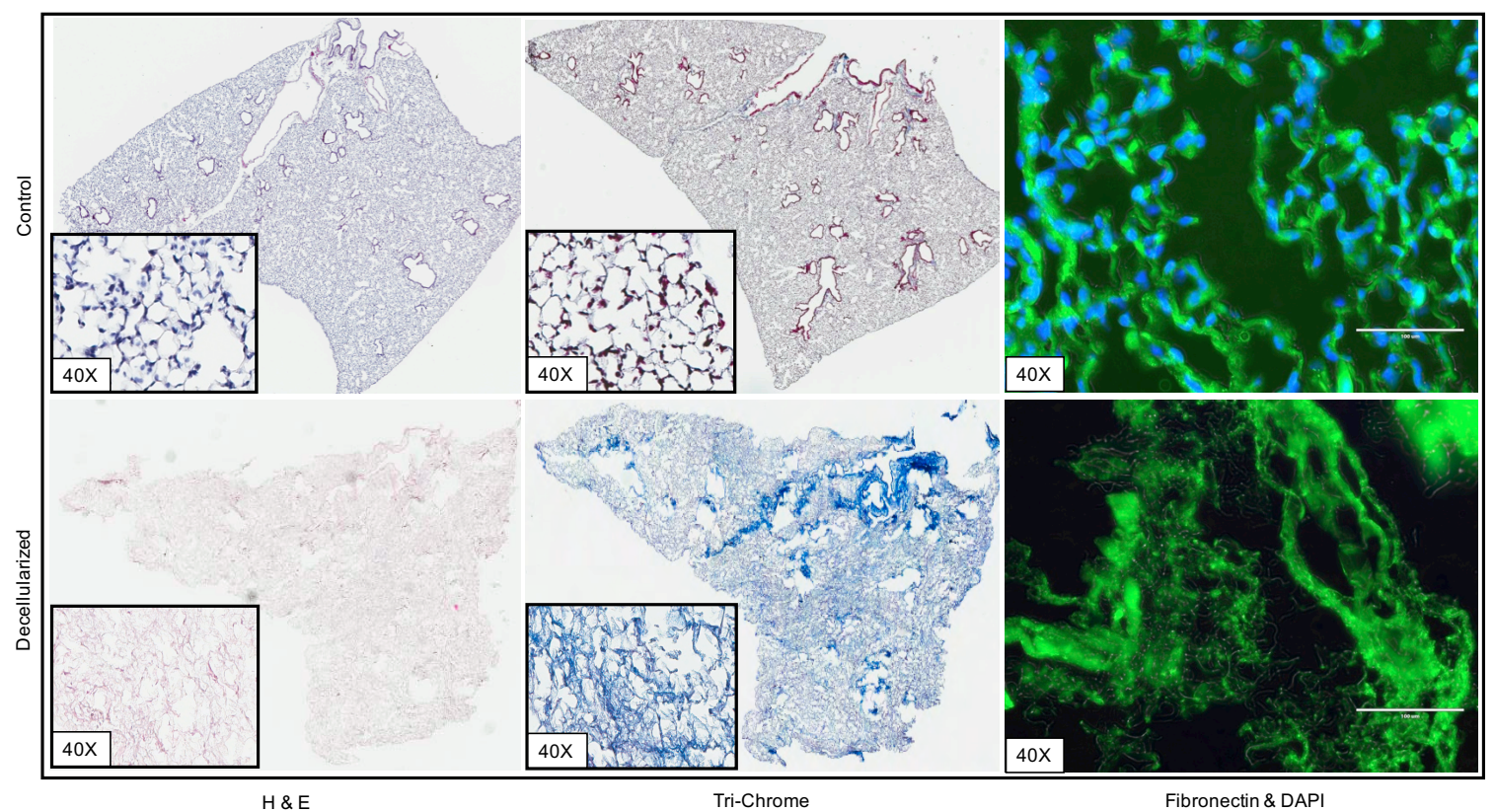

Figure 32. Verification of Acellular Lung

Vicary et. Al., Unpublished

Acellular and normal lung sections from wildtype mice were stained using hematoxylin and eosin, Masson's tri-chrome, or immunohistochemistry. Immunostaining was carried out with control antibodies and antibodies against Fibronectin, DAPI, and IgG. Staining showed removal of cellular material with extracellular matrix proteins in normal architecture remaining. 


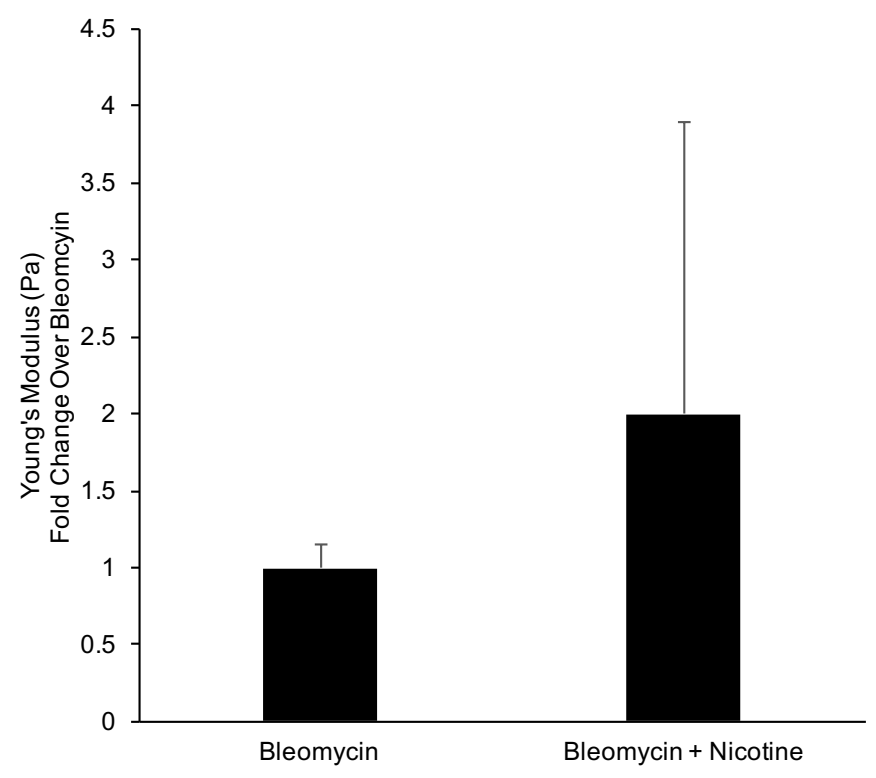

Figure 33. Nicotine Did Not Increased Stiffness of Bleomycin-Treated Lungs

Vicary et. Al., Unpublished

Acellular lungs from bleomycin alone and bleomycin and nicotine treated mice were tested for stiffness by atomic force microscopy. A force map of each sample was conducted and stiffness measurements (pascals) were averaged. There were no significant differences in lung stiffness between bleomycin and bleomycin and nicotine treated mice due to the large variation in stiffness within each lung. Experiments were repeated at least 3 times. 


\subsection{Discussion}

Smoking is considered the most significant contributing factor in several pulmonary diseases, including IPF, but the mechanisms by which these factors induce disease need to be elucidated $[23,25]$. The pathogenesis of pulmonary diseases is intriguing with some patients diagnosed with conditions such as IPF and chronic obstructive pulmonary disease after a relatively brief smoking history, while others can smoke for years with no detrimental health effects. This could be due to the requirement of a second hit or multiple chemical exposures to induce fibrogenesis. Supporting, no studies have shown nicotine alone can induce fibrosis or any other remodeling disease.

Here the data suggesting that nicotine creates an altered ECM state in the lung that accelerating fibrosis caused by a second challenge. Nicotine induces a transitional matrix marked by increased fibronectin, collagen type I, and $\alpha$-SMA expression (Figure 17 and Figure 28) [77]. Fibronectin, collagen, and $\alpha$-SMA are extracellular matrix proteins implicated in tissue injury and disrepair [46, 77, 253]. This induction of ECM proteins is mediated by $\alpha 7 \mathrm{nAChRs}$ (Figure 26 and Figure 27). Additionally, immunohistochemistry analysis showed increased expression of fibronectin throughout the lung, indicating that nicotine is able to induce fibronectin expression in several resident lung cell types, not just fibroblasts, which are normally considered the main producer of ECM proteins (Figure 28). Interestingly, nicotine can also induce fibronectin in an indirect fashion, via BAL fluid (Figure 29). 
Although others and I have shown that nicotine induces ECM proteins, they are at a subclinical level, not marked by structural changes (Figure 29).

Investigating the role of nicotine-induced transitional matrix in pulmonary fibrosis induced by injury, mice were exposed to nicotine for 90 prior to bleomycin instillation. We found nicotine significantly induced bleomycin-induced fibrosis in wildtype mice (Figure 30 ). This suggests that the increased collagen and fibronectin deposition caused by nicotine exposure accelerated fibrosis instigated by a secondary challenge. The role of $\alpha 7$ nAChRs in mediating this effect is unclear. Previously, lack of $\alpha 7 \mathrm{nAChR}$ has been shown to cause increased fibroblast proliferation and airway hyperplasia in $\alpha 7 \mathrm{nAChR}$ deficient mice [238, 254]. This may explain the increased fibrosis seen in untreated $\alpha 7 \mathrm{KO}$ mice when compared to wildtype mice (Figure 30 ). $\alpha 7 \mathrm{KO}$ mice treated with nicotine ablated the increased fibrosis, although the mechanism is unclear.

Lastly, we investigated potential changes in the mechanobiology of the lung caused by nicotine exposure. The lung continuously expanding and contracting, exposes pulmonary cells to rhythmic stress and strain, which are necessary for cellular homeostasis [201]. Changes in tissue stiffness have been shown in COPD and IPF [59, 191-194, 255, 256]. Acellular lungs were isolated from bleomycin alone and nicotine and bleomycin-treated mice. Removal of cellular material while maintaining ECM structure was verified by lack of DAPI staining (Figure 32). Using atomic force microscopy, no differences in lung stiffness were detected between the two groups (Figure 33). This was due to the large variability in lung stiffness seen within lungs. 
In conclusion, we present evidence that nicotine induces subtle changes in lung composition through ECM induction via $\alpha 7 \mathrm{nAChRs}$. This increase in proteins causes significant acceleration of pulmonary fibrosis caused by injury, but the role of $\alpha 7 \mathrm{nAChR}$ and lung stiffness are unclear. Clinically, $\alpha 7 \mathrm{nAChR}$ or ECM degradation might pose promising targets for the prevention of pulmonary fibrosis in current and former smokers. 


\section{CHAPTER V}

\section{THE IMPACT OF CHRONIC NICOTINE EXPOSURE ON SURVIVAL AFTER BLEOMYCIN-INDUCED LUNG INJURY}

\subsection{Introduction}

Approximately 40 million Americans smoke cigarettes, costing the U.S. economy over $\$ 175$ billion a year in healthcare expenditures $[257,258]$. Tobacco smoke is a risk factor for several pulmonary diseases characterized by tissue remodeling, including idiopathic pulmonary fibrosis (IPF). Studying how tobacco smoke promotes these diseases is hindered by tobacco smoke's complexity, containing over 7,000 chemicals $[26,27]$.

Due to the rapid rise of e-cigarettes, research has recently began focusing on nicotine, the main addictive component in tobacco smoke $[259,260]$. Nicotine interacts with cells via cholinergic receptors capable of signal transduction termed nicotinic acetylcholine receptors (nAChRs) [35, 238]. These receptors are multimeric acetylcholine-triggered channel proteins that form homomeric and heteromeric $\alpha$ and $\beta$ chain pentameters [261]. 
During embryogenesis, nicotine- $\alpha 7 \mathrm{nAChR}$ interactions influence lung branching morphogenesis and result in airway thickening and dysfunction $[34,35$, 91]. Nicotine has been shown to induce ECM proteins (e.g. collagen type I, fibronectin, $\alpha-S M A)$, a hallmark of tissue remodeling in lung diseases $[47,77]$. This ECM induction is mediated by $\alpha 7 \mathrm{nAChRs}$ in lung fibroblasts (Figure 10, Figure 12, and Figure 24) [77]. Nicotine cannot cause clinical remodeling (i.e. fibrosis) alone, thus, we set out to investigate the effects of chronic nicotine exposure after a secondary insult using the model of bleomycin-induced lung injury.

Bleomycin instillation generates free radicals injuring DNA [262, 263], lipids [264], and proteins [265], which drive robust inflammation the first two weeks closely resembling acute lung injury (ALI) [171, 266, 267].This phase is followed formation of pulmonary fibrosis during the third week post-instillation [171]. Pulmonary fibrosis has historically been characterized by extensive extracellular remodeling, but more recent research has connected immune responses [118121]. Activated fibroblasts being highly responsive to additional growth factors/proinflammatory cytokines $[118,119]$. This mechanism suggests a capacity for the immune system to accelerate fibroblast-mediated pulmonary remodeling.

Additionally, oxidants have implicated in pulmonary fibrosis [146]. Superoxide, one of the main oxidants in the lung, has been shown to stimulate fibroblasts to secrete collagens, a hallmarks of pulmonary fibrosis [268]. To protect against oxidant damage, the lung has a high abundance of anti-oxidants, including superoxide dismutases (SODs). Overexpression of superoxide dismutase 3 (SOD3) was protective against fibrosis in the bleomycin model [153]. How tobacco 
(i.e. nicotine) affects anti-oxidants is still being elucidated. Some have postulated that cigarette smoke promotes the induction of oxidant stress [146]. In rats, nicotine alone has been shown to affect the oxidative state of the lung by increasing the activity of superoxide dismutase, an important protective mechanism in lung [151, 152].

We hypothesized that nicotine would augment pulmonary injury in wildtype bleomycin-treated animals and that this effect would be ablated in $\alpha 7 \mathrm{nAChR}$ deficient animals $(\alpha 7 K O)$. Mice began dying $\sim 12$ days post bleomycin instillation, but surprisingly we found that nicotine was protective against death, and this protective effect was dependent on the presence of $\alpha 7 \mathrm{nAChR}$. Additionally, an increase in the anti-oxidant SOD1 expression and activity was detected with nicotine-treated wildtype mice, suggesting a potential mechanism of protection. In conclusion, our studies suggest that nicotine- $\alpha 7 \mathrm{nAChR}$ interactions could play an important role in protection against death caused by bleomycin-induced lung injury.

\section{$\underline{5.2 \text { Results }}$}

Nicotine is protective in high-dose bleomycin-induced lung injury by acting on $\alpha 7$ nAChRs

To test the role of chronic nicotine exposure in the bleomycin model, wildtype and $\alpha 7 \mathrm{KO}$ mice were exposed to nicotine $(100 \mu \mathrm{g} / \mathrm{ml})$ in their drinking water for 90 days followed by intratracheal instillation of bleomycin $(2.0 \mathrm{U} / \mathrm{kg})$. This dose of bleomycin was associated with significant mortality with $80 \%$ of animals 
dying within 12 days after bleomycin instillation (Figure 34A). Interestingly, nicotine exposure was protective with $40 \%$ fewer animals dying in response to bleomycin. Even more interesting was the observation that the protective effects of nicotine appeared dependent on $\alpha 7 \mathrm{nAChR}$ expression since animals deficient in this receptor also experienced high mortality despite nicotine treatment (Figure 34B). Similar observations were made in animals exposed to nicotine for a longer term (207 days) (Figure 34C).

\section{Nicotine did not affect bleomycin-induced weight loss or lung fibrosis}

We then set out to investigate the mechanisms responsible for the protective effects of nicotine. First, we examined the body weight of the animals and found that, as predicted, bleomycin-induced weight loss, but this was not affected by nicotine exposure or $\alpha 7 \mathrm{nAChR}$ expression (Figure 35). Second, we examined the histology of the lungs of the surviving mice. We noted no differences between the groups, independent of treatment (Figure 36 and Figure 37).

\section{Inflammation does not appear to play a role in the protective role of nicotine in bleomycin-induced lung injury}

Third, we examined the mRNA expression of soluble mediators involved in inflammation (IL-1 $\beta$ and TNF- $\alpha$ ) and fibrosis (TGF- $\beta$ ) in the surviving animals. As depicted in Figure 38, both nicotine and bleomycin were observed to stimulate the expression of all three mRNAs, but the expression pattern did not suggest inflammation as the cause of nicotine-induced protection. Interestingly, lack of $\alpha 7$ 
$\mathrm{nAChR}$ was associated with increased expression of all three mRNAs at baseline, but the general pattern of mRNA expression was not greatly affected by nicotine or bleomycin.

Nicotine induces superoxide dismutase 1 (SOD1) and appears to be mechanism of the protection in high-dose bleomycin-induced lung injury

Fourth, considering several lines of evidence implicating superoxides as one of the ROS that mediate lung injury, we examined SOD expression [146]. SOD1 has been shown to mediate asbestosis-induced pulmonary fibrosis through production of $\mathrm{H}_{2} \mathrm{O}_{2}$ [269]. In vitro, we found that nicotine stimulates the expression of SOD1 in cultured primary lung fibroblasts (Figure 39). Additionally, presented in Figure $40 \mathrm{~A}$ and $\mathrm{B}$, nicotine and bleomycin were found to increase SOD1 mRNA expression in wildtype, but was absent in $\alpha 7 \mathrm{nAChR}$ KO mice. Lastly, we found nicotine-treated mice had increased SOD activity when compared to non-nicotinetreated (Figure 41). 

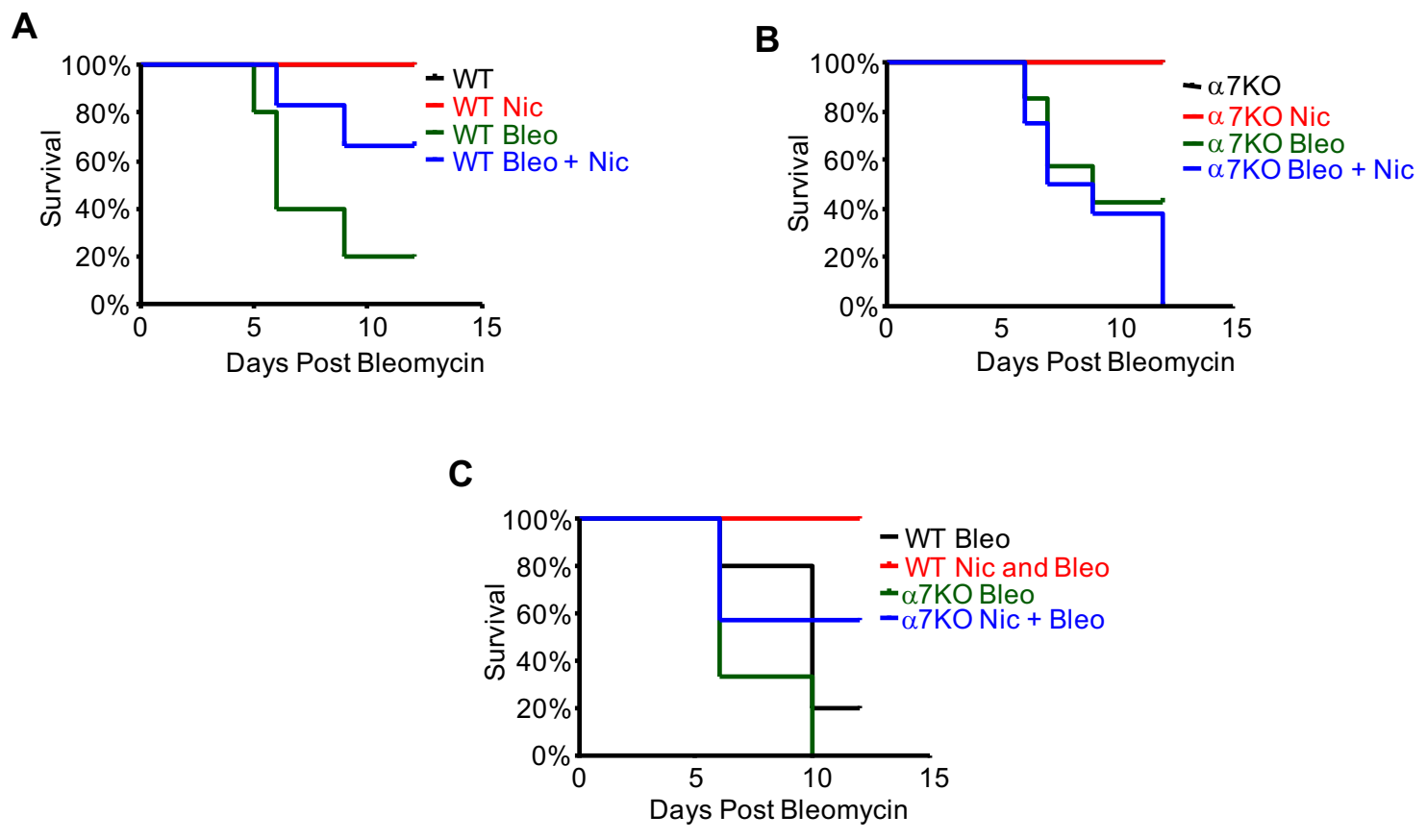

Figure 34. Nicotine Induces Protection Against Dead Caused by BleomycinInduced Lung Injury

Vicary et. Al., Unpublished

(A and B) Wildtype and $\alpha 7 \mathrm{nAChR}$ deficient ( $\alpha 7 \mathrm{KO})$ mice were exposed to nicotine $(100 \mu \mathrm{g} / \mathrm{ml})$ in the drinking water for 107 days then instilled with intratracheal bleomycin $(2.0 \mathrm{U} / \mathrm{kg})$. Death occurred in $80 \%$ of wildtype mice at 12 days post instillation. Nicotine induced significant protection in wildtype mice but was absent in $\alpha 7 \mathrm{KO}$ mice. Wildtype and nicotine-treated mice had $0 \%$ mortality, with wildtype survival line hid behind the nicotine treated mice. (C) Wildtype and $\alpha 7 \mathrm{KO}$ mice were exposed to nicotine $(100 \mu \mathrm{g} / \mathrm{ml})$ in the drinking water for 207 days then instilled with intratracheal bleomycin $(2.0 \mathrm{U} / \mathrm{kg})$. Death occurred in $80 \%$ of wildtype mice at 12 days post instillation. Nicotine induced protection in wildtype mice but there was also small amount of protection in $\alpha 7 \mathrm{KO}$ mice. 
A

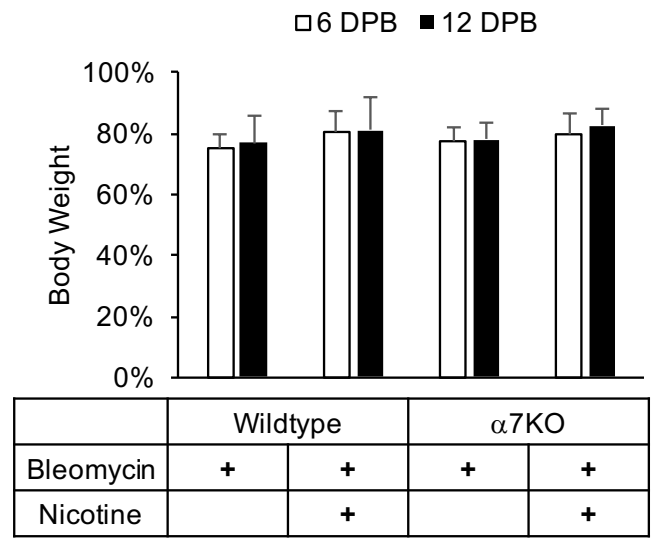

B

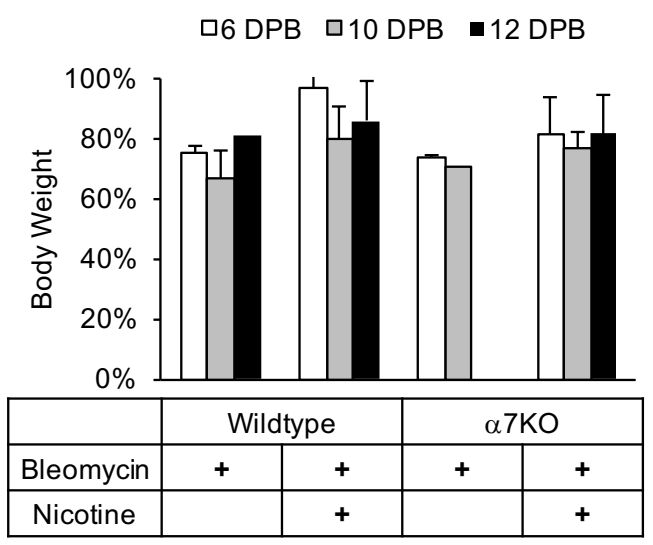

Figure 35. Nicotine-Treatment Does Not Affect Bleomycin-Induced Weight Loss

Vicary et. Al., Unpublished

(A and B) Wildtype and $\alpha 7 \mathrm{nAChR}$ deficient ( $\alpha 7 \mathrm{KO})$ mice were exposed to nicotine $(100 \mu \mathrm{g} / \mathrm{ml})$ in the drinking water for (A) 107 or (B) 207 days then instilled with intratracheal bleomycin $(2.0 \mathrm{U} / \mathrm{kg})$. There was no significant difference in weight lost between the groups tested in A and B. Experiments were repeated at least 3 times. Experiments were repeated at least 3 times. Significance was assessed using $p$ values $<0.05$ obtained by 2 -way ANOVA with Bonferroni posttest. 
A

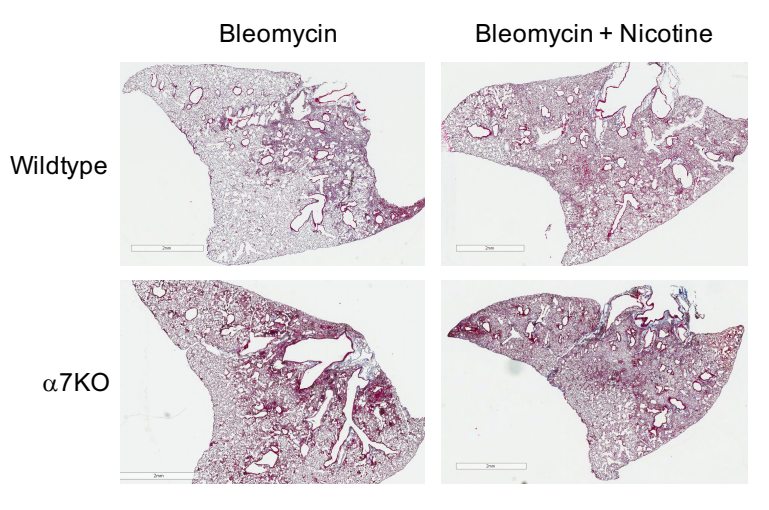

B

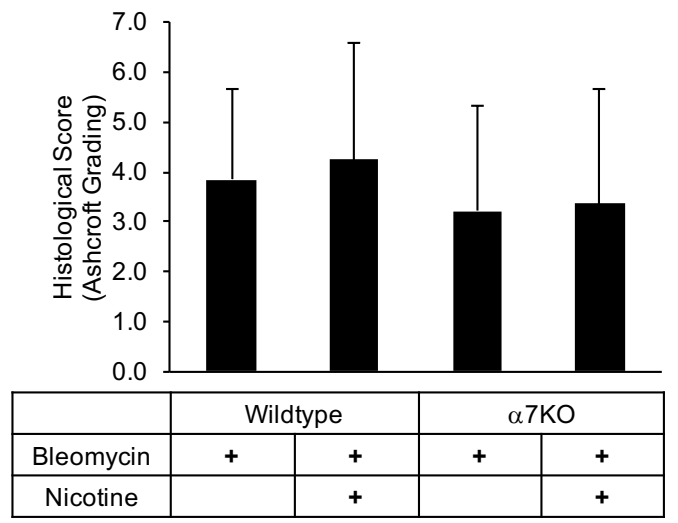

Figure 36. Nicotine-Induced Protection (107 Days) Against BleomycinInduced Death Showed No Differences in Fibrosis

Vicary et. Al., Unpublished

(A) Surviving mice from Figure 34A and B had their lungs inflated at standard pressure, fixed in formalin, paraffin-embedded, and sectioned for histological analysis. Lung sections were stained with hematoxylin and eosin. (B) The trichrome slides were blindly graded on the Ashcroft scale. No significance was found between groups. Experiments were repeated at least 3 times. Significance was assessed using $p$ values $<0.05$ obtained by 2 -way ANOVA with Bonferroni posttest. 
A

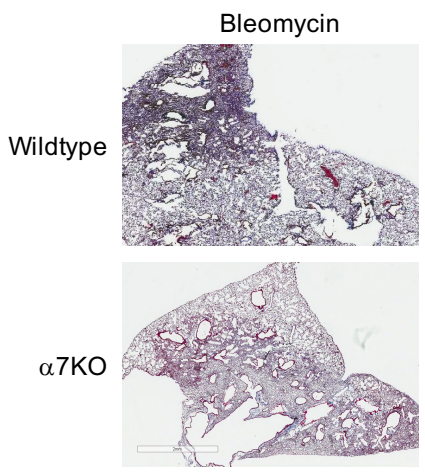

Bleomycin + Nicotine

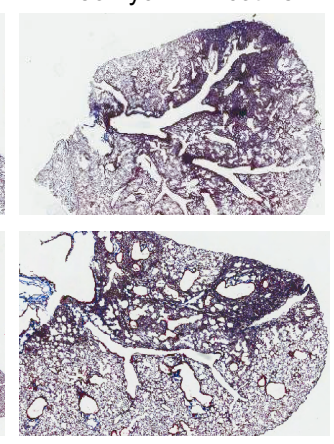

B

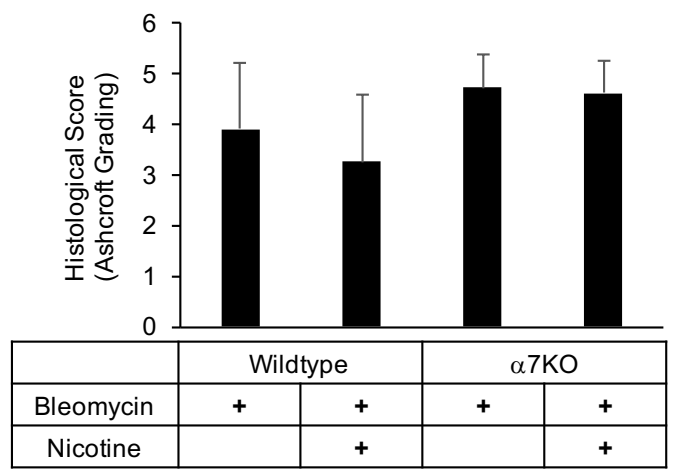

Figure 37. Nicotine-Induced Protection (207 Days) Against BleomycinInduced Death Showed No Differences in Fibrosis

Vicary et. Al., Unpublished

(A) Surviving mice from Figure $34 \mathrm{C}$ had their lungs inflated at standard pressure, fixed in formalin, paraffin-embedded, and sectioned for histological analysis. Lung sections were stained with hematoxylin and eosin. (B) The tri-chrome slides were blindly graded on the Ashcroft scale. No significance was found between groups. Experiments were repeated at least 3 times. Significance was assessed using $p$ values $<0.05$ obtained by 2 -way ANOVA with Bonferroni posttest. 
A

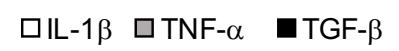

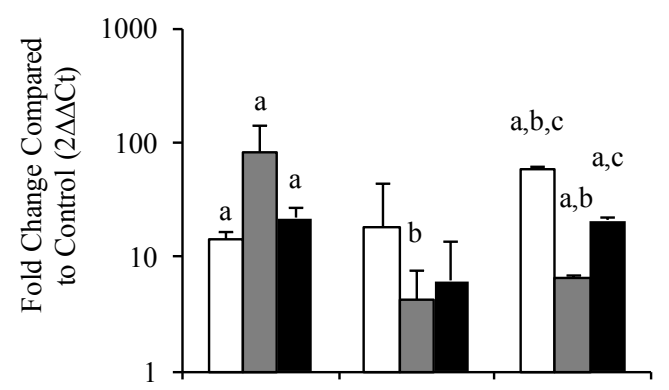

\begin{tabular}{|c|c|c|c|}
\hline & \multicolumn{3}{|c|}{ Wildtype } \\
\hline Nicotine & + & & + \\
\hline Bleomycin & & + & + \\
\hline
\end{tabular}

B

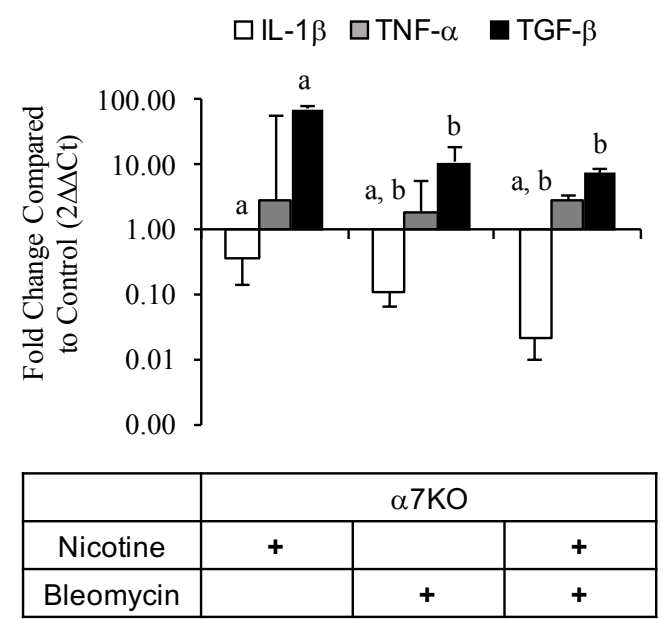

Figure 38. Nicotine-Induced Protection Against Bleomycin-Induced Death Not Connected to Inflammation

Vicary et. Al., Unpublished

(A) We examined the mRNA expression of soluble mediators involved in inflammation (IL-1 $\beta$ and TNF- $\alpha$ ) and fibrosis (TGF- $\beta$ ) in the surviving animals. In wildtype mice, both nicotine and bleomycin stimulated the expression of all three mRNAs, but the expression pattern did not suggest inflammation as the cause of nicotine-induced protection. Untreated wildtype controls normalized have expression equal to 1. (B) Lack of $\alpha 7 \mathrm{nAChR}$ was associated with increased expression of all three mRNAs at baseline, and this general pattern of expression was not greatly affected by nicotine or bleomycin. Untreated $\alpha 7 \mathrm{KO}$ controls normalized have expression equal to 1 . Experiments were repeated at least 3 times. Significance was assessed using $p$ values $<0.05$ obtained by 2 -way ANOVA with Bonferroni posttest. $\mathrm{a}=$ compared to control, $\mathrm{b}=$ compared to nicotine, $\mathrm{c}=$ compared to bleomycin 


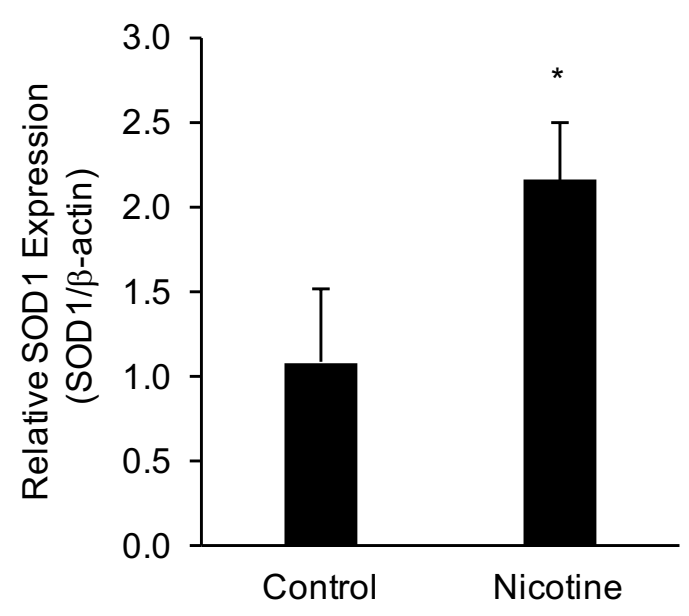

Figure 39. Nicotine-Induces Superoxide Dismutase 1 (SOD1) mRNA Expression

Wildtype lung fibroblasts were cultured in nicotine $(50 \mu \mathrm{g} / \mathrm{ml})$ for 24 hours and then isolated for mRNA. SOD1 expression was significantly upregulated in nicotinetreated fibroblasts. Experiments were repeated at least 3 times. Significance was assessed using $p$ values $<0.05$ obtained by two-tailed t-test. 
A

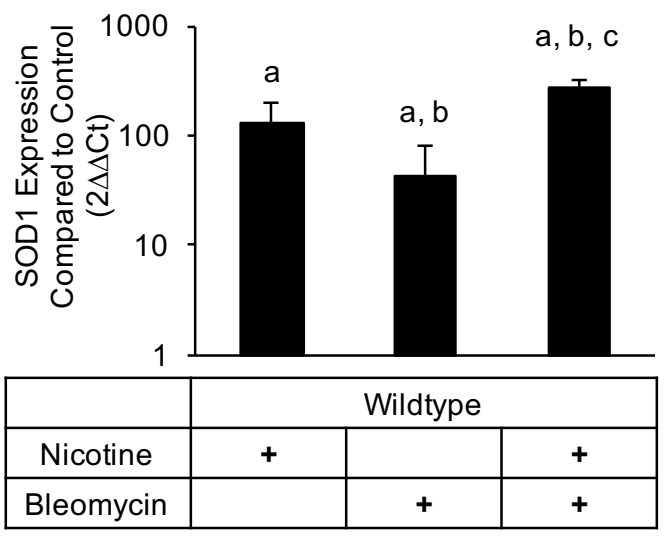

B

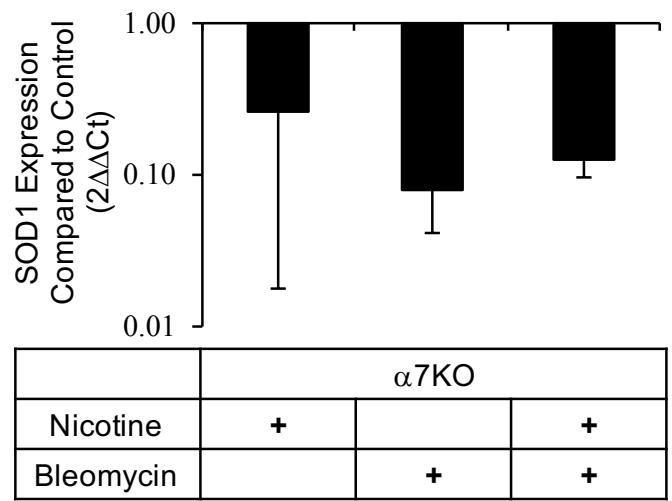

Figure 40. Nicotine-Induces SOD1 mRNA Expression and Activity In Vivo

Vicary et. Al., Unpublished

(A and B) Whole lung mRNA isolated from wildtype and $\alpha 7 \mathrm{KO}$ mice treated with nicotine and/or bleomycin showed significant induction of SOD1 in all three treatment groups. Nicotine and bleomycin had significantly more expression then nicotine or bleomycin alone. Untreated wildtype and $\alpha 7 \mathrm{KO}$ control mice were normalized to have expression equal to 1 . Induction of SOD1 was blunted in the $\alpha 7 K O$ groups. Significance was assessed using $p$ values $<0.05$ using 2-way ANOVA with Bonferroni posttest. $a=$ compared to control, $b=$ compared to nicotine, $\mathrm{c}=$ compared to bleomycin 


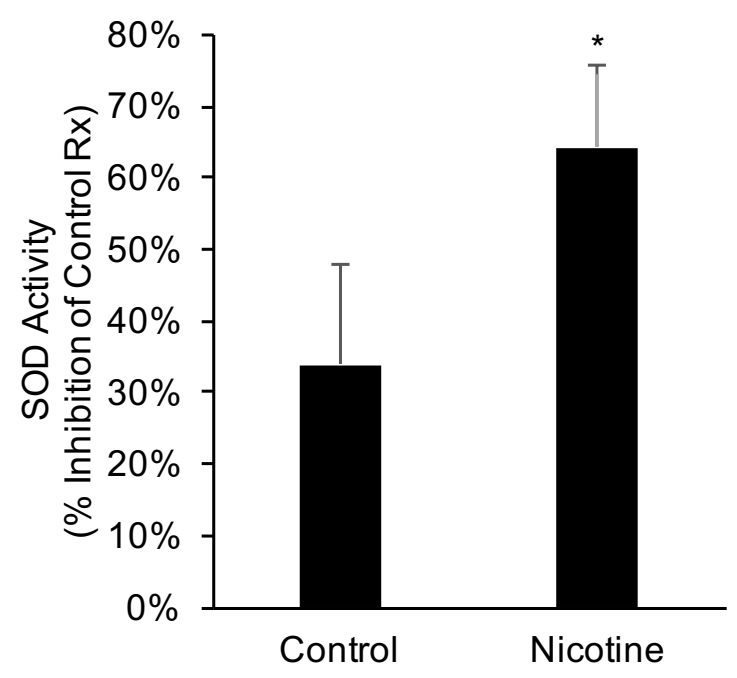

Figure 41. Nicotine-Induces SOD1 Activity In Vivo

In vivo activity of superoxide dismutase was determined using an SOD enzymatic assay. Nicotine-treated mice significantly increased SOD activity when compared to control mice. Experiments were repeated at least 3 times. Significance was assessed using $\mathrm{p}$ values $<0.05$ obtained by two-tailed $\mathrm{t}$-test. 


\section{$\underline{5.3 \text { Discussion }}$}

We set out to investigate the impact of chronic nicotine exposure on bleomycin-induced lung injury. It is well known that bleomycin-induced tissue injury is greatly affected by method of injection, timing, and batch of drug. Routes of injection include inhalational, intratracheal, intraperitoneal, intravenous, and subcutaneous [270]. During the first two weeks after injection, bleomycin induces a robust inflammatory response including IL-1 $\beta$, TNF- $\alpha$ and IL-16 [271]. Bleomycin also induces an intense oxidant response characterized by increased SOD, catalase, glutathione peroxidase malondialdehyde [272-277].

Considering reports by us and others showing nicotine induces extracellular matrix proteins involved in pulmonary fibrosis, we predicted that nicotine would worsen outcomes $[35,77,238]$. Surprisingly, we found that chronic nicotine exposure induced protection against death in mice exposed to bleomycin (Figure 34). Perhaps the most important observation we found was that the protective effect of nicotine was mediated through $\alpha 7 \mathrm{nAChRs}$ since the mortality of animals lacking these receptors was the same as that for wildtype animals. Thus, activation of cholinergic signaling through $\alpha 7 \mathrm{nAChRs}$ resulted in protection against death in the setting of bleomycin.

The protective effect of nicotine was early (12 days) after bleomycin administration suggesting effects during the late inflammatory stage of injury and not related to the fibrotic process seen later in these animals (Figure 36). Thus, we speculated that nicotine affect the inflammatory response. Nicotine has been 
shown to have anti-inflammatory effects on macrophages by reducing the expression of pro-inflammatory cytokines [278-280]. In contrast, we found increased IL-1 $\beta$ and TNF- $\alpha$ expression in the lungs of nicotine-treated animals, suggesting nicotine may stimulate inflammatory cytokines in lung (Figure 38). The cellular origins of these cytokines were not investigated during these studies. However, the $\alpha 7 \mathrm{nAChR}$ seems to play a role in mediating an inflammatory response, with $\alpha 7 \mathrm{KO}$ mice having no or reduced induction of inflammatory cytokines IL-1 $\beta$ and TNF- $\alpha$.

Lastly, investigating other potential mechanisms of protection, we found an increase in SOD1 expression and activity associated with nicotine exposure (Figure 39 and Figure 40). Previous research supports the protective effects of anti-oxidants, with overexpression of SOD and inhalation of lecithinized SOD shown to be protective against bleomycin-induced fibrosis [153, 281]. Additionally, SOD1 has been shown to attenuate DNA breakage induced by bleomycin in vitro [282]. Other SODs, such as EC-SOD (SOD 3) are also protective against bleomycin-induced fibrosis [153]. This mechanism may also be relevant clinically, since Pirfenidone, an FDA approved drug for pulmonary fibrosis, has been shown to rescue SOD loss in renal proximal tubular cells [283].

It should be highlighted that we only tested chronic nicotine exposure, which is likely to elicit responses that differ from those triggered by acute nicotine exposure. Not much research has been conducted on acute nicotine exposure, but the lung is a short-term depot for nicotine after exposure, with the lungs showing 
higher levels than the brain [28]. Additionally, acute nicotine exposure raises cotinine levels similar to chronic exposure, but doesn't affect lung function [284]. Although these observations point to the involvement of $\alpha 7$ nAChRs and anti-oxidants mediating the protective effects of nicotine, we were unable to determine exactly how chronic nicotine exposure protected mice against death caused by bleomycin-induced lung injury. 
CHAPTER VI

\section{A NEW DIAGNOSTIC PHYSIOLOGICAL FORMULA FOR EARLIER DECTECTION OF PULMONARY FIBROSIS PROGRESSION IN IPF PATIENTS}

\section{$\underline{6.1 \text { Introduction }}$}

Idiopathic pulmonary fibrosis (IPF) is defined progressive bilateral fibrotic changes of unknown etiology [1, 5]. IPF affects over 100,000 patients in the U.S. and hundreds of thousands across the world with a dismal survival rate of 3 years from the time of diagnosis $[2,6,285]$. IPF is difficult to diagnose; characterized by impaired lung function and presence of usual interstitial pneumonia, often needing several doctors for confirmation [11, 12, 286]. The number of IPF cases appears to be increasing; due in part to the aging population, increasing patient and physician awareness, and improving computer tomography (CT) imaging [10]. In addition to diagnosis, monitoring IPF severity and progression is critical to decision-making for treatment, management and timing for lung transplantation. 
Currently two new drugs to treat IPF are on the market, but they are not curative, only slowing the progression of fibrosis in some individuals $[287,288]$. The definitive treatment for IPF is lung transplantation in individuals who qualify [5]. The progression of fibrosis varies greatly between patients, with some remaining stable for years, while others decline rapidly [5]. Determination of disease progression in IPF is multifactorial including reported and objective measures of exertional tolerance, radiological changes, and measurement of lung function [289]. Early evidence of pulmonary fibrosis might be manifested by a decrease in lung capacity and reduced diffusing capacity for carbon monoxide, which is typical of fibrosing lung disorders. A six-minute walk test (6MWT) with a portable oximeter should also be considered as hypoxemia may only be detected during exertion in early stages $[5,290]$. Chest imaging is important in confirming IPF diagnosis with high-resolution computed tomography (HRCT) being the best imaging for IPF confirmation.

Forced vital capacity (FVC) is the one spirometry measure of lung function testing that best correlates with disease progression [291, 292]. A $10 \%$ decline in FVC from the baseline measure at diagnosis is considered a significant worsening and is a major end-point in clinical trials in IPF $[50,51]$. The FVC alone, however, is a surrogate only for the restrictive impairment produced by lung fibrosis and manifested through increased elastic recoil of the fibrotic tissue compared to normal lung.

There has been little attention paid to airflow abnormalities in IPF and other lung fibrosing disorders from a clinical standpoint. In addition to FVC, spirometry 
can also measure airway obstruction by forced expiratory volume in 1 second (FEV1) [293]. A FEV1/FVC ratio (Tiffeneau-Pinelli index) has been found to correlate with survival in IPF, but is rarely used clinically [294, 295]. Despite the lack of attention on airflow changes in IPF, a few studies have suggested that airflow abnormalities may be a key determinants of survival in this disease [296298]. High lung elastic recoil is believed to make it difficult to blow expiration fast and consequently makes FEV1/FVC high [299]. Indeed, bronchiolar fibrosis and inflammation seen in histological specimens of individuals with IPF and other forms of pulmonary fibrosis have been known for decades and correlate with impairments in airflow [300-302]. Brown and colleagues found a significantly lower FEV1/FVC in their 5-year survivor group compared to those who died or received lung transplants [296]. Additionally, Schwartz et al. found decreased survival in IPF patients with higher FEV1/FVC [297]. Finally, Wells and co-workers developed a composite physiologic index for predicting survival in IPF in which the FEV1 is an important variable [298]. These studies indicate that first, airflow abnormalities are important in the pathogenesis of IPF and second, that routine clinical tests that measure both airflow and lung capacities can be used to assess disease severity and prognosis.

These findings, along with the typical radiological features of traction bronchiectasis seen in IPF suggest that abnormalities in the airways and airflow are an important part of the pathogenesis in this disease [12]. Cherniack et. Al. showed that total lung capacity and FVC correlated the severity and extent of cellular infiltration of the alveolar walls [303]. Thus, any disease process that 
affects both lung recoil and bronchial structure should impact both airflow and lung capacity simultaneously. Using the belief that fibrosis causes loss of total lung capacity and lung elasticity, we focused on the comparison between the standard

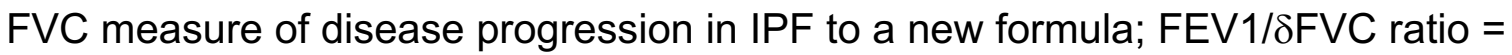
absolute value (FEV1 / (predicted FVC - actual FVC)). The goal of this research is to detect changes in disease progression and severity earlier in the disease course, enhancing clinical decision-making and decreasing the time to progression of end-points in clinical trials (Figure 42).

\section{$\underline{6.2 \text { Results }}$}

\section{Patient Characteristics}

The PANTHER trial yielded 264 patients for analysis. Patients were separated by whether their pulmonary fibrosis progressed over the course of the study (drop in 10\% FVC) (Table 1). Descriptive statistics showed difference in FVC, FEV1 and DLCO \% predicted values. Additionally, CPI and total lung capacity were different.

The cohort of 85 patients (43 placebo/42 treatment) who achieved $\geq 10 \%$

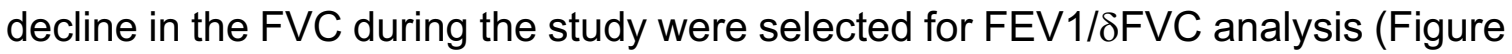
43). Descriptive statistics compared placebo and treatment groups (Table 2). The average age of the patients was 67 years with $82 \%$ of patient's being male. Over $74 \%$ of the patients are current or former smokers. 


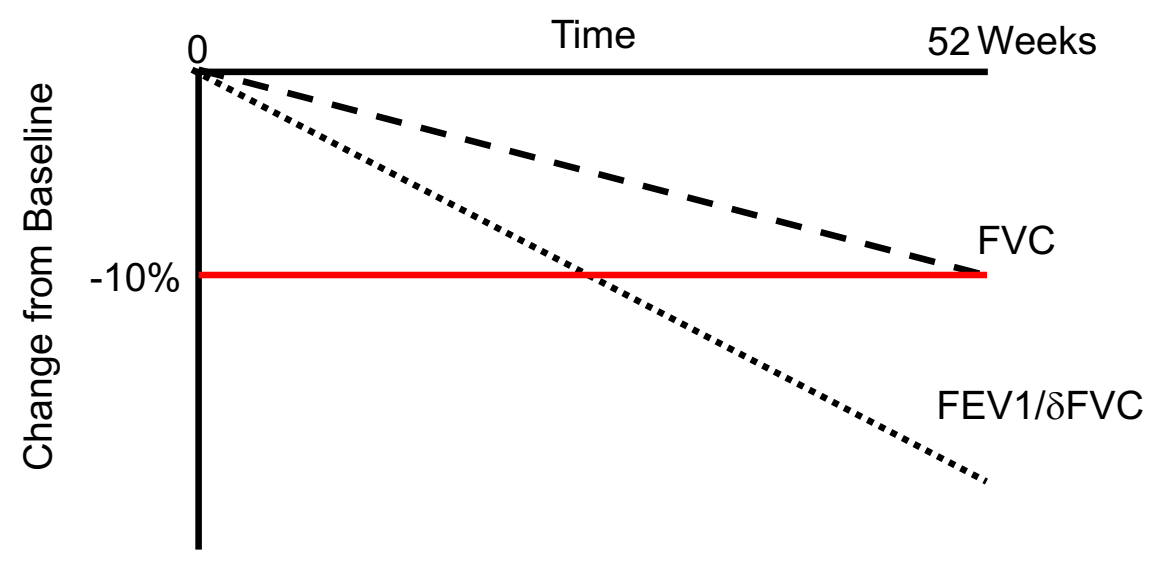

Figure 42. Comparing FEV1/8FVC ratio to FVC Alone

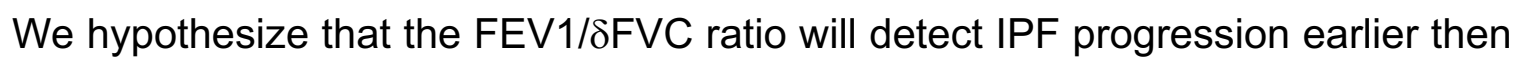
FVC alone. Progression is marked by a decline of $10 \%$ from the patient's baseline value. Using the belief that fibrosis causes loss of total lung capacity and lung elasticity, we compare the standard FVC measure of disease progression in IPF to $\mathrm{FEV} 1 / \delta \mathrm{FVC}$ ratio to detect changes in disease progression and severity earlier in the disease course, enhancing clinical decision-making and decreasing the time to progression of end-points in clinical trials.

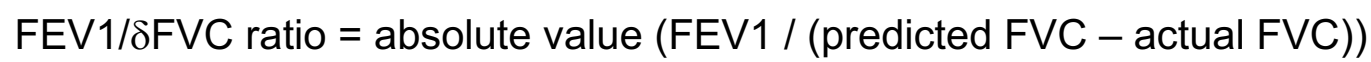




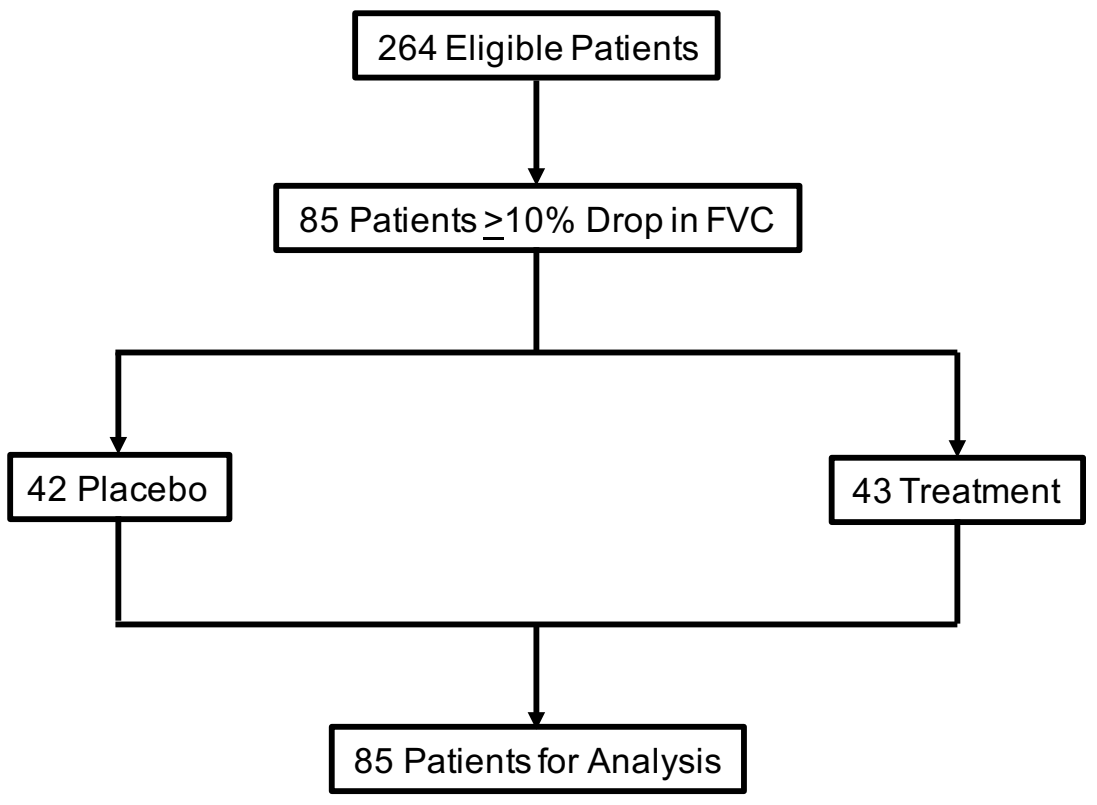

Figure 43. PANTHER Trial Enrollment and Outcomes

Vicary et. Al., Unpublished

Patients with IPF between the ages of 35-85 years who had mild- tomoderate lung-function impairment (defined as a forced vital capacity of $\geq 50 \%$ and a predicted carbon monoxide diffusing capacity of $\geq 30 \%$ were eligible. Patients had received the diagnosis of IPF on the basis of high-resolution computed tomography (CT) or biopsy 48 months or less before enrollment. The PANTHER trial database provided 240 patients for analysis. Individuals who achieved $\geq 10 \%$ decline in the FVC during the course of the study were selected for analysis; 42 placebo and 43 treatment patients. No difference was detected in FVC between the placebo and treatment groups, so all 85 patients were included in statistical analysis. 


\begin{tabular}{|c|c|c|c|}
\hline Variable & $\begin{array}{c}10 \% \text { FVC } \\
\text { Drop } \\
(\mathrm{N}=85)\end{array}$ & $\begin{array}{l}\text { No } 10 \% \\
\text { FVC Drop } \\
(\mathrm{N}=179)\end{array}$ & P-value \\
\hline \multicolumn{4}{|l|}{ Demographics } \\
\hline Age, Median (IQR) & $68(12)$ & $67(11)$ & 0.501 \\
\hline Sex: male, $\mathrm{n}(\%)$ & $70(82)$ & $135(75)$ & 0.268 \\
\hline Race: non-white, n (\%) & $5(6)$ & $7(4)$ & 0.531 \\
\hline Hispanic ethnicity, $\mathrm{n}(\%)$ & $3(4)$ & $11(6)$ & 0.559 \\
\hline \multicolumn{4}{|l|}{ Smoking History } \\
\hline Current smoker, n (\%) & $1(1)$ & $7(4)$ & 0.443 \\
\hline Former smoker, $\mathrm{n}(\%)$ & $63(74)$ & $123(69)$ & 0.47 \\
\hline Never smoked, $\mathrm{n}(\%)$ & $21(25)$ & $48(27)$ & 0.765 \\
\hline \multicolumn{4}{|l|}{ IPF Progress } \\
\hline Duration of IPF (Years), Median (IQR) & $0.5(1.1)$ & $0.8(1.3)$ & 0.087 \\
\hline Years since CT diagnosis of IPF, Median (IQR) & $0.3(0.9)$ & $0.5(1.2)$ & 0.037 \\
\hline $\begin{array}{l}\text { Years since lung biopsy diagnosis of IPF, Median } \\
\text { (IQR) }\end{array}$ & $0.5(1.1)$ & $0.6(1.3)$ & 0.342 \\
\hline \multicolumn{4}{|l|}{ Base Lab Values } \\
\hline FEV1, Median (IQR) & $2.3(0.7)$ & $2.4(0.8)$ & 0.088 \\
\hline FEV \% Predicted, Median (IQR) & $73.2(18)$ & $79.1(22.1)$ & 0.003 \\
\hline FVC, Median (IQR) & $2.9(1)$ & $2.9(1)$ & 0.193 \\
\hline FVC \% Predicted, Median (IQR) & $67.9(16.1)$ & $73.6(21.1)$ & 0.012 \\
\hline FEV1/FVC\%, Median (IQR) & $77.2(2.5)$ & $77.4(2.1)$ & 0.929 \\
\hline FEV1/dFVC, Median (IQR) & $2.6(1.4)$ & $2.8(2.1)$ & 0.199 \\
\hline Alveolar Volume, Median (IQR) & $3.8(1.2)$ & $4(1.3)$ & 0.057 \\
\hline Inspired Volume, Median (IQR) & $2.7(0.9)$ & $2.9(1.2)$ & 0.063 \\
\hline DLCO Actual, Median (IQR) & $12.2(4.8)$ & $13.1(5)$ & 0.063 \\
\hline DLCO Corrected, Median (IQR) & $12(4.8)$ & $13.2(5.3)$ & 0.137 \\
\hline DLCO \% Predicted, Median (IQR) & $41.6(12.2)$ & $45.4(14.3)$ & 0.034 \\
\hline CPI, Median (IQR) & $54(12.4)$ & $49.1(19)$ & 0.006 \\
\hline Alveolar PO2, Median (IQR) & $98.7(5.6)$ & $99.6(7.7)$ & 0.229 \\
\hline Arterial PO2, Median (IQR) & $82.1(11.1)$ & $82(16.8)$ & 0.628 \\
\hline Arterial PCO2, Median (IQR) & $39(4.1)$ & $38(5)$ & 0.142 \\
\hline Arterial SO2, Median (IQR) & $95.7(2.3)$ & $95.8(3)$ & 0.505 \\
\hline Alveolar-Arterial Gradient, Median (IQR) & $17.3(8.9)$ & $17.4(15.3)$ & 0.992 \\
\hline Total Lung Capacity, Median (IQR) & $4.1(1.4)$ & $4.4(1.5)$ & 0.032 \\
\hline Predicted Total Lung Capacity, Median (IQR) & $6.6(1.2)$ & $6.6(1.4)$ & 0.512 \\
\hline
\end{tabular}

\section{Table 1. Drop in 10\% FVC Patient Cohort Baseline Characteristics}

Vicary et. Al., Unpublished The PANTHER trial patients were separated by whether their idiopathic pulmonary fibrosis (IPF) progressed over the course of the study (drop in 10\% FVC). Differences in FVC, FEV1 and DLCO \% predicted values were detected. Additionally, CPI and total lung capacity showed statistical differences. CT = Computer Tomography, FEV1 = Forced Expiratory Volume in One Second, FVC = Forced Vital Capacity, DLCO = Diffusing Capacity of the Lungs for Carbon Monoxide, $\mathrm{CPI}=$ Composite Physiologic Index 


\begin{tabular}{|c|c|c|c|}
\hline Variable & $\begin{array}{l}\text { Placebo } \\
(\mathrm{N}=\mathbf{4 2})\end{array}$ & $\begin{array}{c}\text { Treatment } \\
\text { (N=43) }\end{array}$ & P-value \\
\hline \multicolumn{4}{|l|}{ Demographics } \\
\hline Age, Median (IQR) & $65.5(12.2)$ & $69(11)$ & 0.076 \\
\hline Sex: male, $n(\%)$ & $33(79)$ & $37(86)$ & 0.407 \\
\hline Race: non-white, n (\%) & $1(2)$ & $4(9)$ & 0.36 \\
\hline Hispanic ethnicity, n (\%) & $1(2)$ & $2(5)$ & $>0.999$ \\
\hline \multicolumn{4}{|l|}{ Smoking History } \\
\hline Current smoker, n (\%) & $1(2)$ & $0(0)$ & 0.494 \\
\hline Former smoker, $\mathrm{n}(\%)$ & $31(74)$ & $32(74)$ & $>0.999$ \\
\hline Never smoked, $\mathrm{n}(\%)$ & $10(24)$ & $11(26)$ & $>0.999$ \\
\hline \multicolumn{4}{|l|}{ IPF Progress } \\
\hline Duration of IPF (Years), Median (IQR) & $0.5(1.2)$ & $0.5(1)$ & 1 \\
\hline Years since CT diagnosis of IPF, Median (IQR) & $0.3(0.9)$ & $0.3(0.9)$ & 0.665 \\
\hline $\begin{array}{l}\text { Years since lung biopsy diagnosis of IPF, Median } \\
\text { (IQR) }\end{array}$ & $0.5(1)$ & $0.5(1.1)$ & 0.969 \\
\hline \multicolumn{4}{|l|}{ Base Lab Values } \\
\hline Baseline FEV1, Median (IQR) & $2.3(0.7)$ & $2.3(0.8)$ & 0.476 \\
\hline Baseline FEV \% Predicted, Median (IQR) & $74.7(18.1)$ & $72.9(18.6)$ & 0.585 \\
\hline Baseline FVC, Median (IQR) & $3(1.1)$ & $2.9(0.9)$ & 0.318 \\
\hline Baseline FVC \% Predicted, Median (IQR) & $68.9(14.6)$ & $67.4(20.1)$ & 0.465 \\
\hline Baseline FEV1/FVC\%, Median (IQR) & $77.8(2.5)$ & $76.9(2.3)$ & 0.146 \\
\hline Baseline FEV1/dFVC, Median (IQR) & $2.7(1.2)$ & $2.5(1.4)$ & 0.299 \\
\hline Baseline Alveolar Volume, Median (IQR) & $3.9(1.4)$ & $3.6(1.1)$ & 0.286 \\
\hline Baseline Inspired Volume, Median (IQR) & $2.9(1)$ & $2.7(0.7)$ & 0.389 \\
\hline Baseline DLCO Actual, Median (IQR) & $11.8(4.7)$ & $12.3(4.5)$ & 0.906 \\
\hline Baseline DLCO Corrected, Median (IQR) & $11.9(4.8)$ & $12.3(4.8)$ & 0.885 \\
\hline Baseline DLCO \% Predicted, Median (IQR) & $40.9(10.3)$ & $42(12.8)$ & 0.796 \\
\hline Baseline CPI, Median (IQR) & $53.9(12.6)$ & $55.1(12.6)$ & 0.573 \\
\hline Baseline Alveolar PO2, Median (IQR) & $97.2(6.4)$ & $99.1(5)$ & 0.703 \\
\hline Baseline Arterial PO2, Median (IQR) & $82.2(12.9)$ & $82.1(10)$ & 0.662 \\
\hline Baseline Arterial PCO2, Median (IQR) & $39(4)$ & $39.2(5)$ & 0.675 \\
\hline Baseline Arterial SO2, Median (IQR) & $96(2.7)$ & $95.3(1.4)$ & 0.622 \\
\hline Baseline Alveolar-Arterial Gradient, Median (IQR) & $17.3(10.7)$ & $16.9(8.9)$ & 0.544 \\
\hline Baseline Total Lung Capacity, Median (IQR) & $4.1(1.6)$ & $4.1(1.2)$ & 0.671 \\
\hline Predicted Total Lung Capacity, Median (IQR) & $6.6(1.3)$ & $6.6(0.8)$ & 0.874 \\
\hline
\end{tabular}

\section{Table 2. FEV1/8FVC Ratio Patient Cohort Baseline Characteristics}

Vicary et. Al., Unpublished

The cohort of 85 idiopathic pulmonary fibrosis (IPF) patients who achieved $>10 \%$ decline in the FVC during the study were compared for baseline characteristics in placebo versus treatment group. No significant differences were detected between the placebo and treatment groups. CT = Computer Tomography, FEV1 = Forced Expiratory Volume in One Second, FVC = Forced Vital Capacity, DLCO = Diffusing Capacity of the Lungs for Carbon Monoxide, CPI = Composite Physiologic Index 


\section{Spirometry Value Distribution}

The patient cohort displayed Gaussian distribution for baseline values of FEV1, FVC and FEV1/FVC ratio (Figure 44). Baseline values included an average FEV1 of 2.3 liters (L) (73.7\% predicted), average FVC of 3 liters $(68 \%$ predicted) and an average $\% \mathrm{FEV} 1 / \mathrm{FVC}$ of $77.26 \%$. Average baseline value of FEV1/אFVC was 2.6 with two outliers skewing the histogram (one with $\sim 600$, another with 93). By nature of the formula, it is possible for very high numbers to

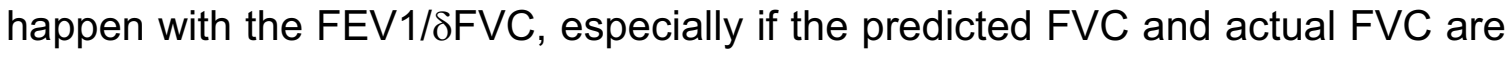
very close.

\section{Primary Outcomes}

Time to $10 \%$ decline in FVC was compared to $10 \%$ decline in FEV1/אFVC.

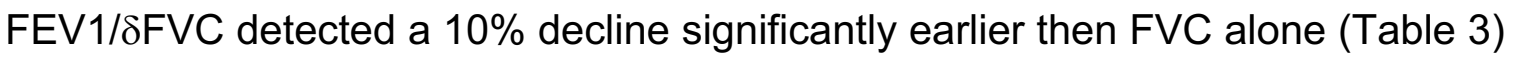
(Stuart-Maxwell Test $\mathrm{X}_{(3)}^{2}=23.58, \mathrm{p}<0.001$ ). The time to $10 \%$ decline in FVC was compared to $10 \%$ drop in FEV1/8FVC (Figure 45 ). $42.5 \%$ of the patients had a $10 \%$ drop detected in FVC and FEV1/8FVC at the same visit. $25 \%$ of patients were detected 15 weeks earlier, while another $25 \%$ of the patients were detected 30 weeks earlier. Cumulatively, over $50 \%$ of the patients had a $10 \%$ drop in

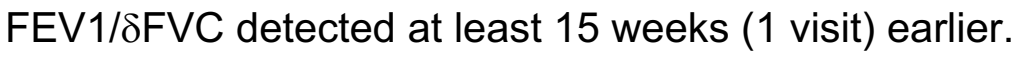

\section{Secondary Outcomes}

Investigating the potential role of spirometry values on clinical outcomes, a drop in $10 \%$ FVC and FEV1/8FVC ratio were analyzed as predictors for re- 
hospitalization and death. An increase in deaths was detected in the cohort who dropped more than $10 \%$ in their FVC (Table 4). No differences found between control and treatment cohorts using the FEV1/8FVC ratio were found (Table 5). Additionally, Kaplan-Meier comparison curves were computed for death/hospitalization using FVC alone or FEV1/SFVC, but neither showed enough efficacy to be used clinically (Figure 46). Lastly, patients were compared weekly for placebo vs treatment or those who had a \%FVC drop at the same time as $\%$ FEV1/8FVC vs later. Neither comparison showed a significant difference (Figure 47). 
A

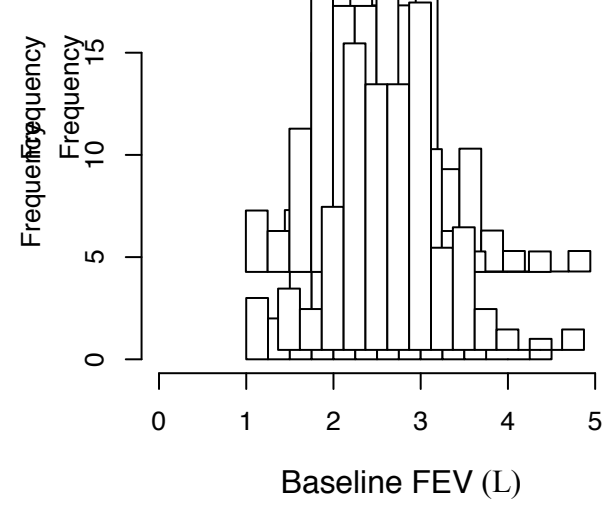

$\mathrm{C}$

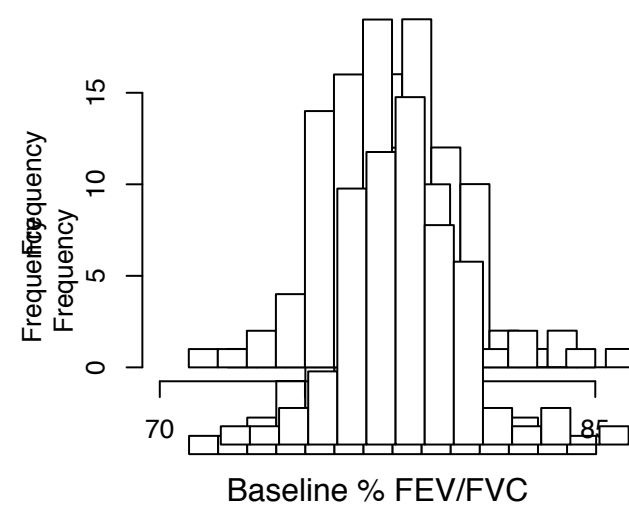

B

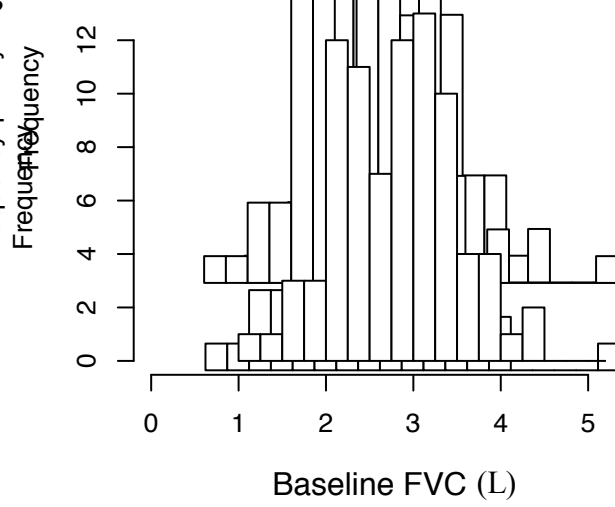

$\mathrm{D}$

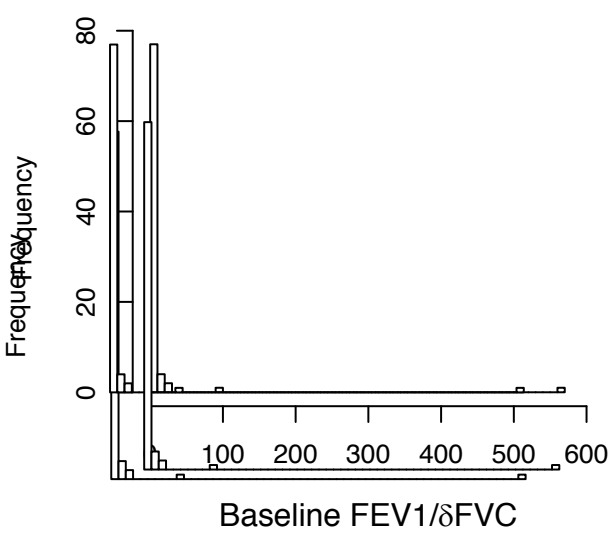

Figure 44. Distribution of FEV1, FVC, FEV1/FVC and FEV1/8FVC Values

Vicary et. Al., Unpublished

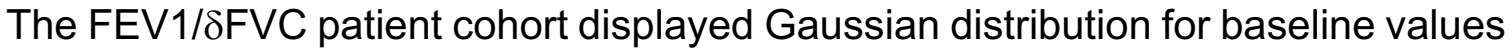
of FEV1, FVC and FEV1/FVC ratio. (A) Baseline values included an average FEV1 of 2.3 liters $(\mathrm{L})(73.7 \%$ predicted) with a range from 1-5 L. (B and C) Patients had an average FVC of 3 liters (68\% predicted) with a range of 1-5 $L$ and an average

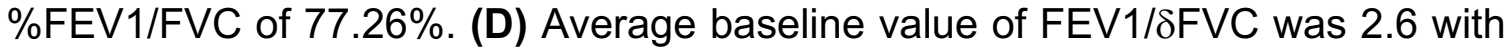
two outliers skewing the histogram (one with $\sim 600$, another with $\sim 93$ ). 


\begin{tabular}{|c|c|c|c|c|c|c|}
\hline \multicolumn{7}{|c|}{ OVERALL } \\
\hline & \multicolumn{6}{|c|}{ Time to $10 \%$ loss FVC } \\
\hline \multirow{6}{*}{ 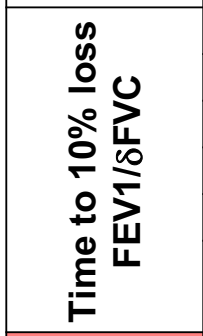 } & & 15 weeks & 30 weeks & 45 weeks & 60 weeks & Row Total \\
\hline & 15 weeks & 17 & 12 & 16 & 7 & 52 \\
\hline & 30 weeks & 0 & 7 & 7 & 5 & 19 \\
\hline & 45 weeks & 0 & 0 & 9 & 2 & 11 \\
\hline & 60 weeks & 0 & 0 & 0 & 3 & 3 \\
\hline & $\begin{array}{c}\text { Column } \\
\text { Total }\end{array}$ & 17 & 19 & 32 & 17 & 85 \\
\hline $\begin{array}{l}\text { FVC } 10 \% \\
\text { loss earlier } \\
\text { by } 3 \text { visits }\end{array}$ & $\begin{array}{l}\text { FVC } 10 \% \\
\text { loss earlier } \\
\text { by } 2 \text { visits }\end{array}$ & $\begin{array}{l}\text { FVC } 10 \% \\
\text { loss earlier } \\
\text { by } 1 \text { visit }\end{array}$ & $\begin{array}{c}10 \% \text { loss } \\
\text { at same } \\
\text { visit }\end{array}$ & $\begin{array}{c}\text { FEV1/8FVC } \\
10 \% \text { loss } \\
\text { earlier by } 1 \\
\text { visit }\end{array}$ & $\begin{array}{c}\text { FEV1/8FVC } \\
10 \% \text { loss } \\
\text { earlier by } 2 \\
\text { visits } \\
\end{array}$ & $\begin{array}{c}\text { FEV1/8FVC } \\
10 \% \text { loss } \\
\text { earlier by } 3 \\
\text { visits }\end{array}$ \\
\hline
\end{tabular}

Table 3. FEV1/8FVC Ratio Decreases by $10 \%$ Earlier Then FVC Alone

Vicary et. Al., Unpublished

Time to $10 \%$ decline in FVC was compared to $10 \%$ decline in FEV1/8FVC.

FEV1/8FVC detected a 10\% decline significantly earlier then FVC alone (StuartMaxwell Test X2(3) $=23.58, p<0.001)$. 


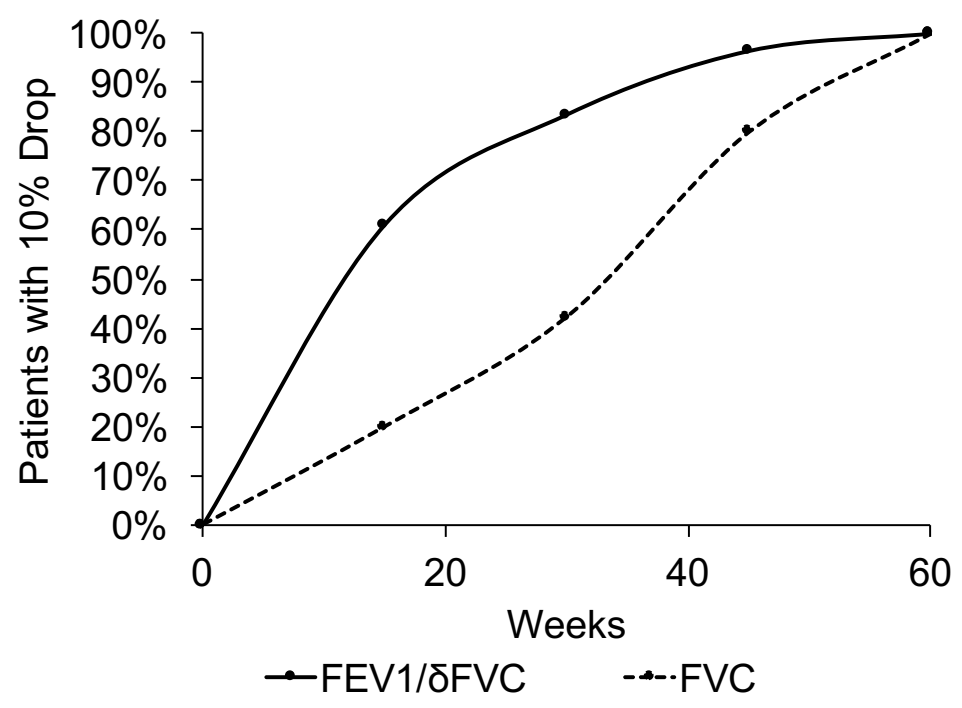

Figure 45. FEV1/8FVC Earlier Detection in Patients by Weeks

Vicary et. Al., Unpublished

The time to $10 \%$ decline in FVC was compared to the time of $10 \%$ drop in FEV $1 / \delta F V C$. Over $75 \%$ of patients had a $10 \%$ decline detected in FEV $1 / \delta F V C$ by 30 weeks ( 3 visits), where a 10\% drop in FVC had been detected in less than $50 \%$ of patients. 


\begin{tabular}{|l|c|c|c|}
\multicolumn{1}{|c}{ Variable } & \multicolumn{1}{c|}{$\begin{array}{c}\text { 10\% FVC } \\
\text { Drop } \\
\text { (N = 85) }\end{array}$} & $\begin{array}{c}\text { No 10\% } \\
\text { FVC Drop } \\
\text { (N=179) }\end{array}$ & P-value \\
\hline Death, n (\%) & $6(7)$ & $3(2)$ & 0.033 \\
\hline Time to Death, Median (IQR) & $64(3.3)$ & $63.9(5)$ & 0.656 \\
\hline Hospitalized, n (\%) & $16(19)$ & $22(12)$ & 0.189 \\
\hline Time to Hospitalization, Median (IQR) & $63.9(15.9)$ & $63.3(11.1)$ & 0.89 \\
\hline Death or Hospitalization, n (\%) & $17(20)$ & $24(13)$ & 0.203 \\
\hline Time to Death/Hospitalization, Median (IQR) & $63.9(15.9)$ & $63.3(11.1)$ & 0.89 \\
\hline
\end{tabular}

Table 4. Drop in $10 \%$ FVC as Predictor of Hospitalization/Death

Vicary et. Al., Unpublished

The PANTHER trial patients were separated by whether their pulmonary fibrosis progressed over the course of the study (drop in 10\% FVC). Predicting rehospitalization and death endpoints showed a significant difference in deaths between patients who did and did not drop in 10\% FVC. 


\begin{tabular}{|l|c|c|c|}
\hline \multicolumn{1}{|c}{ Variable } & $\begin{array}{c}\text { Placebo } \\
\text { (N = 42) }\end{array}$ & Treatment & P-value \\
\hline Death, $\mathrm{n}(\%)$ & $2(5)$ & $4(9)$ & 0.676 \\
\hline From respiratory causes & $2(5)$ & $3(7)$ & $>0.999$ \\
\hline Hospitalized, $\mathrm{n}(\%)$ & $8(19)$ & $8(19)$ & $>0.999$ \\
\hline Death or Hospitalization, $\mathrm{n}(\%)$ & $8(19)$ & $9(21)$ & $>0.999$ \\
\hline \multicolumn{4}{|l|}{ Survival at 60 weeks based on Kaplan-Meier estimator, \% (95\% Cl) } \\
\hline Death & $94.7(87.8-100.0)$ & $89.9(80.9-99.8)$ & 0.441 \\
\hline Hospitalization & $79.2(67.3-93.2)$ & $80.5(69.3-93.7)$ & 0.932 \\
\hline Death or Hospitalization & $79.2(67.3-93.2)$ & $78.5(67.0-92.0)$ & 0.878 \\
\hline
\end{tabular}

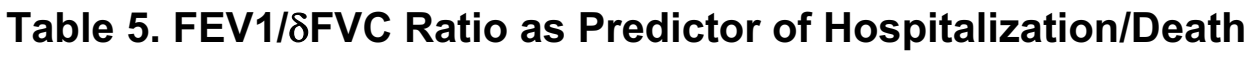

The cohort of 85 patients who achieved $>10 \%$ decline in the FVC during the course of the study were compared for endpoint characteristics in placebo versus treatment group. Predicting re-hospitalization and death endpoints showed no difference between FEV1/אFVC ratio and FVC. 


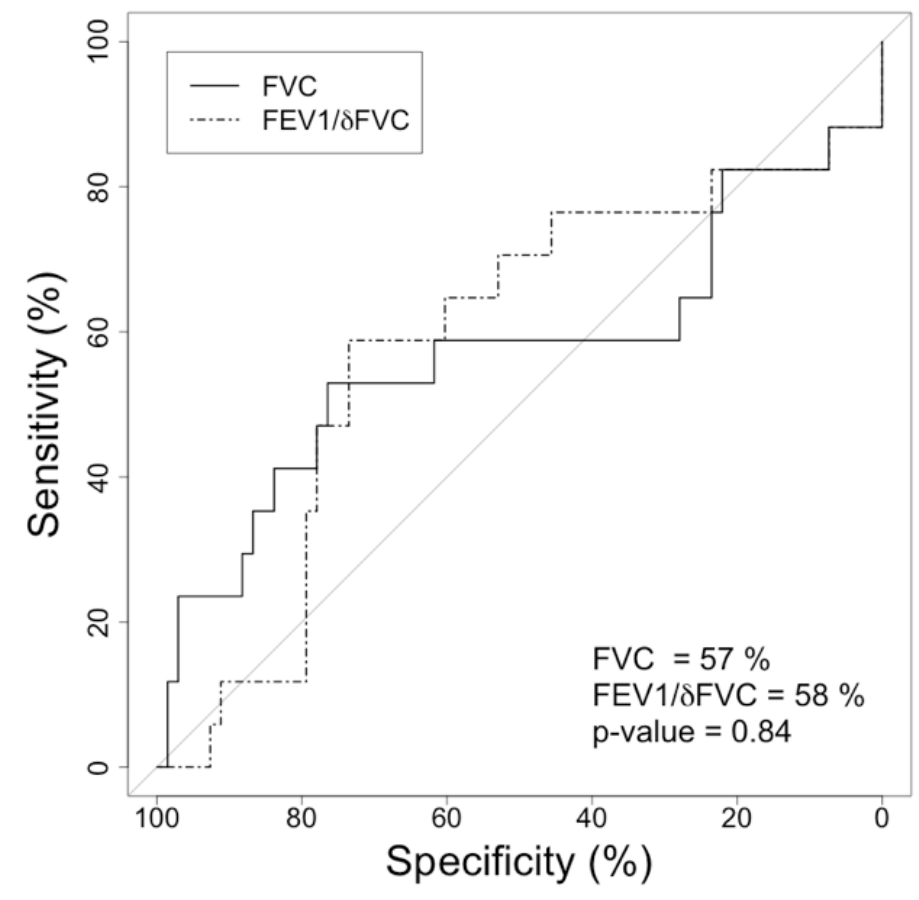

Figure 46. Death and Hospitalization Receiver Operating Characteristics (ROC) Curve of FEV1/8FVC and FVC Alone

Vicary et. Al., Unpublished

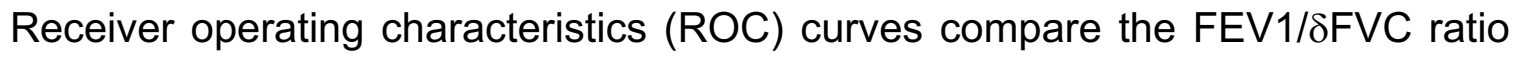
versus FVC alone in regards to sensitivity and specificity as a predictor of death

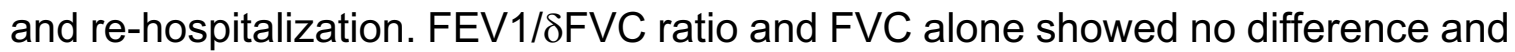
neither were accurate enough predicting death and hospitalizations to be used clinically. 
A

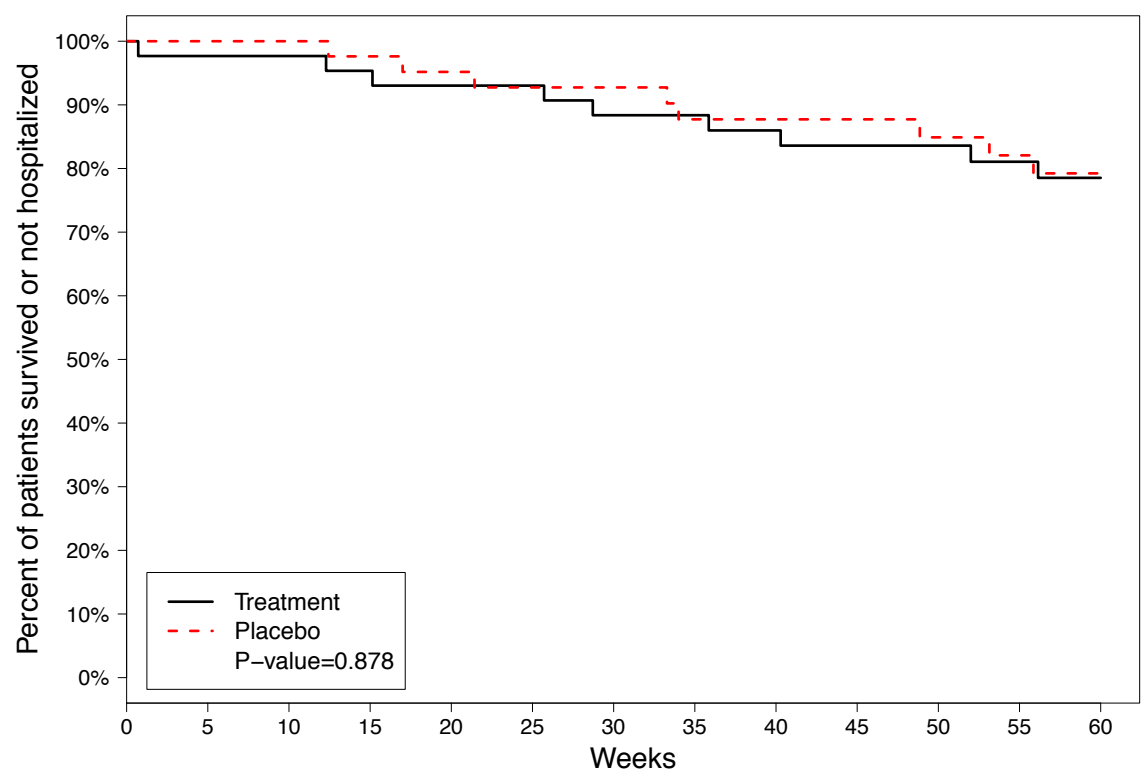

B

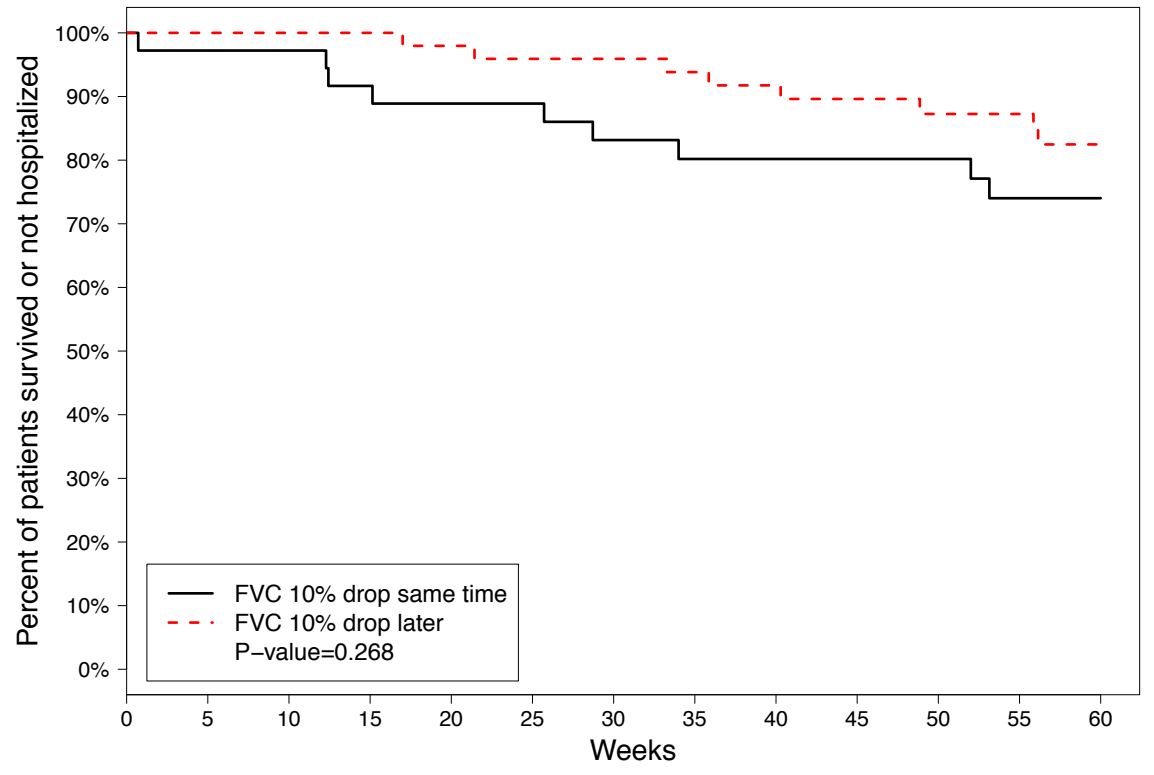

Figure 47. Patient Hospitalization/Death by Weeks

Vicary et. Al., Unpublished

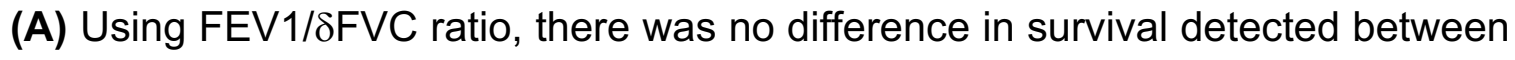
treatment and placebo groups. (B) No difference in survival between $10 \%$ drop in

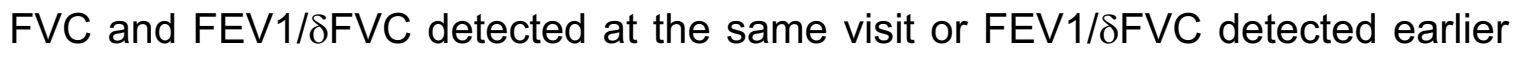
were detected. 


\subsection{Discussion}

With IPF affecting so many people around the world and having an average survival rate of 3 years, the need for better treatments is imperative. The FDA recently approved two drugs for the treatment of IPF and several other drugs are in development $[287,288]$. Creating more sensitive markers for IPF progression can allow drug trials to be completed earlier, allowing for more efficient FDA approval. Better disease markers will also allow physicians to determine the best treatment regimen for patients.

The current paradigm on the progression of pulmonary fibrosis has always included loss of lung volume due to loss of lung elasticity due to the expansion of fibrotic foci, but restrictive airway remodeling has been overlooked. The research presented here and by others show that including restrictive airway measurements might increase the specificity and sensitivity of spirometry as a diagnostic marker for fibrosis progression and survival. Nishiyama et. Al. showed lower FEV1/FVC (below 83\%) was associated with increased patient survival [294]. Additionally, shown here, restrictive airway (FEV1) with lung volume (FVC) allowed for earlier detection of $10 \%$ decline then FVC alone.

There are limitations to this study. Co-morbidities may affect the ratio, such as combined pulmonary fibrosis and emphysema diagnoses which showed to lower the FEV1/FVC ratio [304]. Additionally, only progressive patients (drop $\geq 10 \%$ FVC) were used for analysis. Including non-progressive patients would not have 
allow us to compared our primary endpoints (FEV1/אFVC and FVC) for patients that did not drop $10 \%$ in FVC by the end of the study.

Multiple values and tests may end up being used in clinical practice for measuring progression, including spirometry and biomarkers [305]. Daily FVC values recently showed significance in predicting patient survival [292]. Additionally, the 6-minute walk test (6MWT) has shown to be associated with dyspnea and quality of life scores, but no significant difference was detected between 6MWT and FVC [290]. We hope research presented here will help to advance the ability to track progression of IPF in patients. 


\section{CHAPTER VII}

\section{PULMONARY FIBROSIS IN HERMANSKY-PUDLAK SYNDROME}

\subsection{Introduction}

Pulmonary fibrosis is characterized by the accumulation of fibroblasts and the unrestrained chaotic expression and deposition of connective tissue matrices leading to effacement of the pulmonary architecture with loss of lung function. Environmental factors (e.g. asbestos and silica exposure), genetic factors (e.g. surfactant-related proteins and telomerase), and viral factors (herpesvirus) have been associated with the development of lung fibrosis, while other factors are believed to contribute to its progression (e.g., allergens, tobacco) [1, 13-17]. However, pulmonary fibrosis often occurs in subjects where a cause cannot be identified [1].

Despite the growing association of genetics with pulmonary fibrosis, the mechanisms by which abnormalities in these genes lead to alterations in cellular functions; extracellular matrix expression, deposition and degradation; production of profibrotic growth factors; and, ultimately, disruption of lung homeostasis and pulmonary fibrosis remain poorly understood. 
One genetic disorder associated with highly penetrant pulmonary fibrosis is the Hermansky-Pudlak Syndrome (HPS), a rare autosomal recessive disorder characterized by oculocutaneous albinism, bleeding diathesis, granulomatous colitis, and, in many cases, pulmonary fibrosis. HPS affects approximately 1:500,000-1,000,000 people worldwide, and has been reported in individuals from India, Japan, China, United Kingdom, Western Europe, Pakistan, Canada and the United States [306, 307]; the HPS Network registry also includes patients from Israel, Australia, Hungary, Mexico, Brazil, Peru, Qatar, and Bolivia, among others. Although HPS is a very rare disease, it is relatively common in Puerto Rico, where the prevalence of HPS-1 is estimated to be about $1: 1,800$ in the Northwest region of the island, accounting for approximately $50 \%$ of all cases globally [308]. Unfortunately, the average life expectancy of HPS patients is $40-50$ years and there are currently no effective treatments, with over $70 \%$ of patients dying due to complications directly related to the disease [309].

Here, we summarize the current understanding of the pathogenesis of HPS and its clinical manifestations and management, while discussing recent discoveries that might lead to the identification of potential targets for intervention and effective therapies in the future. This review is intended to raise awareness about pulmonary fibrosis in HPS, and prompt interest in further research in this syndrome, research that could also hold the key for understanding more common pulmonary fibrosing disorders. 


\subsection{Genetic and Functional Abnormalities in Hermansky-Pudlak Syndrome}

(HPS)

HPS was first described in 1959 by Dr. Frantisek Hermansky and Dr. Paulus

Pudlak who identified two patients with oculocutaneous albinism and bleeding diathesis in Czechoslovakia [310]. HPS is a non-sex-linked autosomal recessive disorder with no known disease manifestations in carriers. Ten types of HPS have been defined by nine unique genes distinguished by their signs, symptoms, and genetic cause; and in most cases, each subtype has more than one variant (Table 6) [307, 311-313]. HPS-1 is the most common and generally severe subtype. HPS2 and -4 also have severe manifestations and HPS-10 has neurological changes, while patients with the other variants (HPS-3, 5-9) generally manifest somewhat milder symptoms [314].

In Puerto Rico, HPS-1 and -3 are the most common subtypes. This is because certain populations in Puerto Rico were established by a very small number of individuals who carried the silent gene (founder effect) [308]. One in twenty-one Puerto Ricans in the Northwest of the Island are carriers of the gene mutation (>160,000 people) [315]. Thus, it is estimated that 7-8 children with HPS will be born on the island each year [308]. In Puerto Rico, HPS-1 arises mainly due to a duplication/frameshift (16-base pairs) on exon 15 of the HPS1 gene, accounting for $45 \%$ of all HPS cases globally and most cases in the island [308, 316]. Outside of Puerto Rico, HPS-1 is caused by different mutations within the HPS1 gene loci [317]. Another $25 \%$ of HPS cases in the island occur from a 


\begin{tabular}{|c|c|c|c|c|}
\hline $\begin{array}{c}\text { Human } \\
\text { Type }\end{array}$ & Gene & NCBI & Human & Protein \\
& & RefSeq & $\begin{array}{c}\text { Chromosome } \\
\text { Location }\end{array}$ & Complexes \\
\hline HPS1 & HPS1 & NM_000195 & $10 q 24.2$ & BLOC-3 \\
\hline HPS2 & AP3B1 & NM_003664 & $5 q 14.1$ & AP-3 \\
\hline HPS4 & HPS3 & NM_032383 & 3q24 & BLOC-2 \\
\hline HPS5 & HPS5 & NM_0181507 & $11 \mathrm{p} 15.1$ & BLOC-2 \\
\hline HPS6 & HPS6 & NM_024747 & $10 q 24.32$ & BLOC-2 \\
\hline HPS7 & DTNBP1 & NM_032122 & $6 p 22.3$ & BLOC-1 \\
\hline HPS8 & BLOC1S3 & NM_212550 & $19 q 13.32$ & BLOC-1 \\
\hline HPS9 & BLOC1S6 & NM_012388 & $15 q 21.1$ & BLOC-1 \\
\hline HPS10 & AP3D1 & NM_003938 & $19 p 13.3$ & AP-3 \\
\hline
\end{tabular}

Table 6. Human Hermansky-Pudlak Syndrome (HPS) Variants

HPS is a non-sex-linked autosomal recessive disorder with no known disease manifestations in carriers. Nine types of HPS have been defined by nine unique genes distinguished by their signs, symptoms, and genetic cause. 
deletion (3,904-base pairs) in HPS3. HPS3 is believed to have developed in the $1880-1890$ s in the central mountainous region of Puerto Rico $[308,316,318]$. Consistent with the genetic drift expected over hundreds of years, more recent studies have suggested that carrier frequencies for HPS1 and HPS3 are similar across Puerto Rico [308, 319].

Exactly how the genetic mutations in HPS genes lead to all manifestations of the disease is unknown. HPS genes code for HPS proteins, which form complexes termed biogenesis of lysosome-related organelles complexes (BLOCs) (Figure 48) [320-322]. Lysosome related organelles (LROs) perform specialized functions in the sorting and trafficking of specific cargo. BLOC-3, a complex which includes the HPS1 and HPS4 proteins and others, functions as a Rab32/38 guanine nucleotide exchange factor; these are proteins capable of activating small GTPases, thereby affecting intracellular signaling and downstream targets.

HPS mutations impair the biogenesis and function of LROs, which explains some disease manifestations. For example, LRO dysfunction affecting pigmentproducing cells (termed melanocytes) is responsible for albinism. Specifically, in melanocytes, activated Rab32/38 is needed for the transport of tyrosinase and tyrosinase related protein 1 (TYRP1) from early ribosomes to melanosomes; this likely represents a key step and its disruption has been shown to lead to the oculocutaneous manifestations of HPS-1 [323]. In over $80 \%$ of patients with HPS, this is manifested by lentigines, while a small percentage of patients may also develop solar keratosis, squamous cell, or basal cell carcinomas [315]. Decreased pigmentation in the eyes causes legal blindness in most patients, as well as 


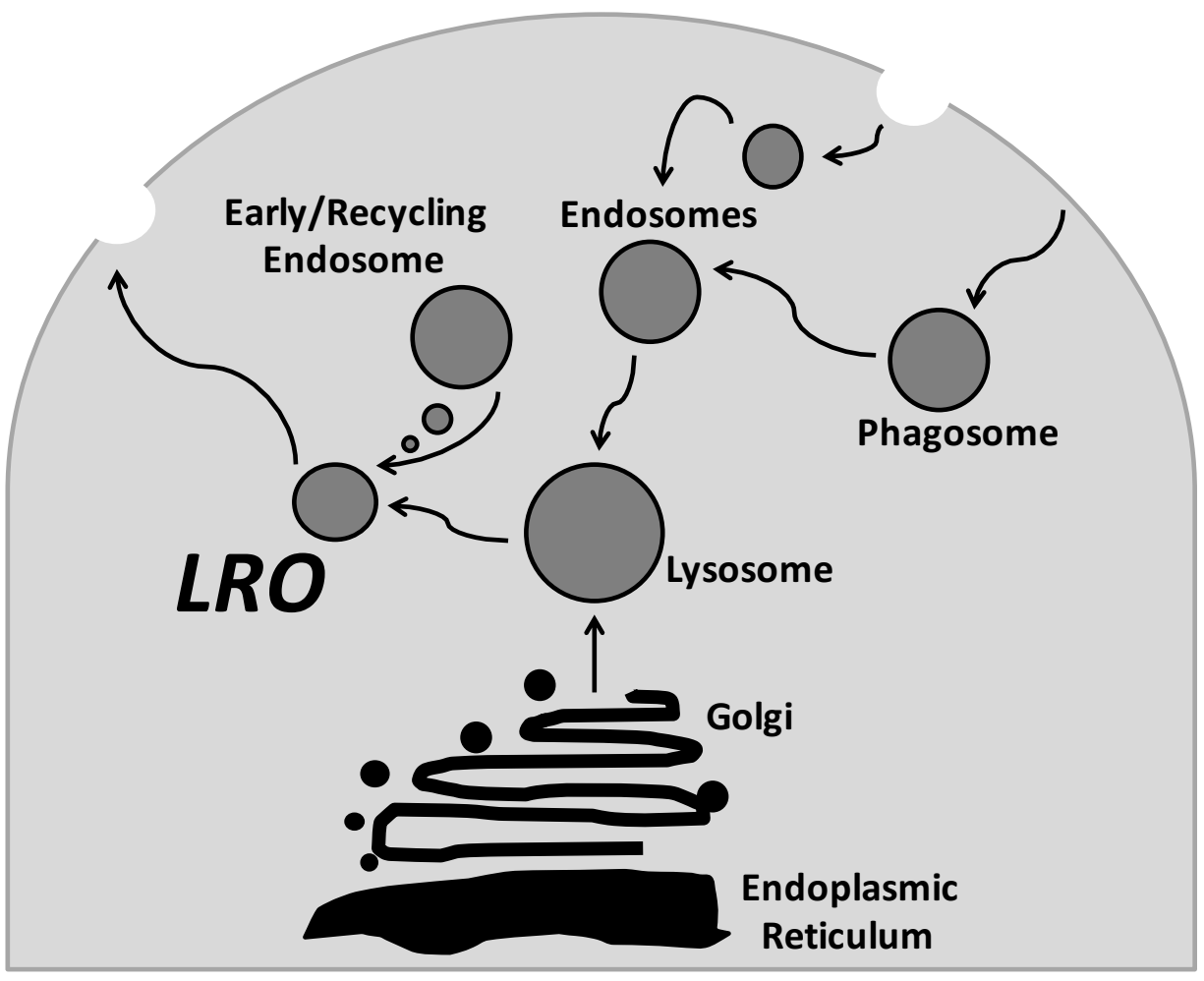

Figure 48. Lysosomal-Related Organelles (LROs) in HPS

Lysosome-related organelles (LROs) are a group of cell type-specific subcellular compartments that share some features with endosomes and lysosome, but also develop unique properties as they harbor specialized cargoes. Many of the clinical manifestations of HPS are explained by abnormalities in the formation and trafficking of LROs, including albinism (melanosomes), bleeding (platelet dense granules), and pulmonary fibrosis (likely related at least in part to abnormal lamellar body genesis in type II alveolar epithelial cells). 
photophobia, impaired dark adaptation, and nystagmus.

Similarly, defects in platelet LROs leads to bleeding diathesis due to inhibition of the formation of platelet dense bodies [324]. In type II alveolar epithelial cells of the lung, Rab38 helps maintain lamellar body morphology and surfactant homeostasis [323]. The role of this defect in the development of pulmonary fibrosis is not known, but it is intriguing to note that abnormalities in surfactant proteins have been identified in idiopathic pulmonary fibrosis and related interstitial lung disorders [323, 325, 326]. Disease mechanisms remain uncertain for the occurrence of granulomatous colitis that has also been reported in approximately $15 \%$ of HPS patients, not necessarily tracking with occurrence of subtype risk for pulmonary fibrosis.

Accumulation of an amorphous lipid-protein complex, ceroids, which increases with age in patients with HPS has been speculated to be a potential trigger for the development of tissue inflammation, possibly contributing to some of the multi-organ manifestations of HPS [315, 322, 327, 328].

\subsection{HPS and Pulmonary Fibrosis (HPS-PF)}

In a joint statement of the American Thoracic Society and European Respiratory Society, HPS-related pulmonary fibrosis (HPS-PF) and idiopathic pulmonary fibrosis (IPF) are considered similar entities (albeit with distinct causes)

since both can show similar histological patterns [41]. IPF occurs spontaneously in most patients, but research has uncovered some genetic mutations in surfactant 
proteins, mucins, and telomerases that are considered relevant [1, 14-17]. Only HPS $-1,-2$ and -4 are associated with the development of pulmonary fibrosis, but $100 \%$ of HPS-1 patients will develop HPS-PF [328]. Both HPS-PF and IPF are characterized by irreversible and progressive fibrosis of the lung parenchyma and interalveolar septa, ultimately leading to death from respiratory failure. Similar symptoms are found in HPS-PF and IPF, including increased dyspnea initially manifested as exercise intolerance that progresses to dyspnea at rest and the need for supplemental oxygen over time.

One intriguing, but important difference between IPF and HPS-PF is the age at which pulmonary fibrosis is detected. Spontaneous IPF manifests in patients mostly over age 50 (this is not the case for familial IPF), while HPS-PF occurs much earlier (30-40 years). Another major difference is that the average survival time after diagnosis differs with IPF carrying a dismal 3-year survival rate of $50 \%$, while HPS patients live approximately 10 years after diagnosis [5, 329]; this might reflect earlier ascertainment and not differing natural history.

Current understanding of the pathogenesis of pulmonary fibrosis points to epithelial cell dysfunction as the inciting cause of the disease. Several intracellular trafficking processes might be affected leading to endoplasmic reticulum stress and apoptosis caused by environmental exposure to injurious agents (e.g., viral infection, recurrent aspiration of gastric contents) or through genetic abnormalities (e.g., surfactant proteins). Injured or dysfunctional epithelial cells are proposed to promote the accumulation of fibroblasts that are responsible for the excessive deposition of extracellular matrices [18]. The origins of these fibroblasts are 
unclear, but combinations of mechanisms are hypothesized, including the transdifferentiation of epithelial cells (epithelial-mesenchymal transformation), the differentiation of fibrocytes and stem cells, and the proliferation of resident fibroblasts [42]. Regardless of their source of origin, the interplay between dysfunctional epithelial cells and activated fibroblasts (also termed myofibroblasts because of their expression of smooth muscle cell markers) seems to drive unrestrained collagen deposition and tissue scarring. Underlying amplifying factors like aging and epigenetic changes are thought to further perpetuate the progressive nature of pulmonary fibrosis [18].

There is a growing body of work dissecting the mechanisms of pulmonary fibrosis in HPS. HPS gene products are ubiquitously expressed and thus many cell types have been potentially implicated as contributors to the pathogenesis of HPS-PF. First, there is strong evidence that macrophage-mediated inflammation precedes pulmonary fibrosis in HPS patients. Bronchoalveolar lavage (BAL) fluid of HPS-PF patients contained increased total cell counts and alveolar macrophages [330], at a time when patients still had relatively preserved lung function. Furthermore, alveolar macrophages from HPS patients have been shown to be constitutively activated, expressing excess cytokines and chemokines when studied ex vivo [330]. BAL fluid from HPS patients also had elevated levels of several cytokines, including chemokine (C-C motif) ligand 2 (CCL2/MCP-1), macrophage colony-stimulating factor (M-CSF), and granulocyte-macrophage colony-stimulating factor (GM-CSF) [330]. Interestingly, elevation of CCL2 levels was directly associated with the progression of HPS-PF; a similar association has 
been observed in IPF and animal models of pulmonary fibrosis [331, 332].

Abnormalities in fibroblasts have also been reported including increased expression of galectin-3, a $\beta$-galactoside-binding lectin with profibrotic effects [333]. In addition, patients with HPS-PF have been found to have increased numbers of circulating CXCR4-positive fibrocytes compared to subjects with HPS without lung disease or healthy control subjects [334]. Increasingly, as suggested for IPF, studies point to the type II alveolar epithelial cell as central in the pathogenesis of HPS pulmonary fibrosis. Lamellar bodies (responsible for producing surfactant proteins) are a type of LRO [326], making alveolar epithelial cells a good candidate for dysfunction in HPS.

As HPS lung tissue is limited in availability for research due to disease rarity and contraindication to lung biopsy because of the bleeding diathesis, much HPS research to date has been conducted in murine models, which also afford opportunities for access to cells and tissues, and genetic manipulations not possible in humans. Murine models of HPS reliably share many aspects of the human disease including hypopigmentation, deficiency of platelet dense granules, and abnormalities of type II alveolar epithelial cells and alveolar macrophages (Table 7 and Table 8). Mice have more than 16 different genes with HPS-like phenotypes [335], though many of the gene functions are unknown. At least six mouse models have the orthologous mutations to the human genes [336-341].

Interestingly, murine models of HPS-1 (Pale ear) and HPS-2 (Pearl) show activation of alveolar macrophages in the lung, but not in the blood or peritoneum [342, 343]. Pale ear HPS1 mice do not develop spontaneous fibrosis, but have 


\begin{tabular}{|c|c|c|}
\hline Mouse Model & Mouse RefSeq & Mouse Chromosome Location \\
\hline Pale Ear & NM_019424 & Chr19 \\
\hline Pearl & NM_009680 & Chr13 \\
\hline Cocoa & NM_080634 & Chr13 \\
\hline Light Ear & NM_138646 & Chr5 \\
\hline Ruby eye-2 & AF534396 & Chr7 \\
\hline Ruby eye & NM_176785 & Chr19 \\
\hline Sandy & NM_025772 & Chr13 \\
\hline Zebrafish & NM_001025476 & Chr15 \\
\hline Pallid & NM_019788 & Chr2 \\
\hline Muted & NM_139063 & Chr13 \\
\hline Cappuccino & NM_133724 & Chr5 \\
\hline Mocha & NM_007460 & Chr10 \\
\hline
\end{tabular}

Table 7. Mouse models of HPS

Vicary et. Al., 2016

There are several murine models of HPS. Each model of HPS has been defined by a unique gene and a name based off the specific characteristics. 


\begin{tabular}{|c|c|}
\hline Physiology/Cellular Abnormality & Mouse HPS Gene(s) \\
\hline Hypopigmentation & All \\
\hline Prolonged Bleeding & All \\
\hline Lung Disease & $\begin{array}{c}\text { HPS1/HPS2 Double Mutant, } \\
\text { Pldn }\end{array}$ \\
\hline Decreased Lysosomal Enzyme Secretion & Most \\
\hline Decreased CTL Granule Secretion & Rab27a \\
\hline Dysregulated Mast Cell Granule Secretion & HPS6 \\
\hline CD1d Deficiency & HPS2 \\
\hline NK Cell Dysfunction & HPS1, Pldn, rp \\
\hline Decreased Proplatelet Production & Rabggta \\
\hline Night Blindness & HPS2 \\
\hline Susceptibility to Anesthetics & Pldn, rp \\
\hline Protection from Atherosclerosis & HPS4, HPS6 \\
\hline Increased Emperipolesis & Rabggta \\
\hline Otolith Deficiency & Ap3d, Pldn, mu \\
\hline Hippocampal Granule Deficiency & Ap3d \\
\hline Decreased Lifespan & Rabggta, HPS2, HPS3, PIdn \\
\hline
\end{tabular}

Table 8. Phenotype and cellular abnormalities in mouse models of HPS

Murine models of HPS reliably share many aspects of the human disease including hypopigmentation, deficiency of platelet dense granules, and abnormalities of type II alveolar epithelial cells and alveolar macrophages. 
higher baseline collagen deposition and show increased inflammation and collagen expression in response to silica challenge [342, 344, 345]. In response to bleomycin, HPS-1 and HPS-2 mice developed fibrosis significantly earlier and to a greater extent than wildtype mice [343]. Also, higher levels of transforming growth factor- $\beta$ were detected in bleomycin-treated HPS mice. However, it is intriguing that spontaneous lung fibrosis does not occur in these single mutant models, suggesting that other factors and secondary hits are required to develop the full manifestations of the disease. With aging, spontaneous fibrosis has been reported in a HPS double-mutant model, generated by mating HPS-1 and HPS-2 mice [346]. Alveolar epithelial cell dysfunction in HPS remains under study, but recent publications implicate alveolar epithelial cell apoptosis [346, 347], Chitinase-3-like-1 and its receptors [348], and autophagy [349] as potential mechanisms. Macrophage-mediated inflammation is also present in HPS mouse models. However, murine bone marrow transplant studies have demonstrated that macrophage abnormalities and fibrotic susceptibility are likely due to epithelial dysfunction with type II alveolar cell death, not intrinsic macrophage defects [347]. This is important because it suggests that HPS-PF could be driven more than just macrophage mutations. Collectively, these studies indicate the role of HPS mice as an experimental model for HPS disease, and identify mechanisms that are likely also relevant for other fibrosing lung diseases. 


\subsection{Diagnosing HPS and HPS-PF}

The diagnosis of HPS includes the documentation of oculocutaneous albinism and platelet storage pool deficiency [328]. HPS patients are potentially identified shortly after birth by their albinism, though the degree of hypopigmentation is variable. Other individuals are not diagnosed until after experiencing other disease complications such as excessive bleeding after circumcision or easy bruising; over $75 \%$ of patients have bruises at the time of clinical examination. Bleeding time is often quite prolonged, but is not recommended for diagnostic or screening due to poor specificity and lack of prognostic value [350]. Ophthalmologists might be the first to suggest testing for HPS due to diminished vision, with transillumination of the iris in addition to nystagmus and strabismus.

Testing for HPS is currently limited by complexity, expense, and availability of specialized diagnostics required for confirmation. The gold standard test for the platelet abnormality in HPS is analysis by whole mount electron microscopy for the absence of dense bodies [351]. Testing of platelet aggregation following platelet stimulation with adenosine diphosphate or epinephrine (using a platelet aggregometer) [352] provides a less specific alternative. Genetic testing plays a growing role in diagnosis, and it is important for determining HPS subtype (and prognosis). Such assessments are also essential to determine the numbers of individuals with distinct HPS subtypes in any given community. However, genetic testing has not been widely implemented for HPS due to the cost of next- 
generation sequencing [353]. Genetic testing could be used to help identify specific mutations as the price of next-generation sequencing decreases. Not all patients with HPS have identified genetic mutations though, suggesting that there are additional HPS disease-causing genes to be discovered. In the absence of genetic confirmation, patients with the classic manifestations of the syndrome (e.g., albinism with bleeding disorder, colitis or pulmonary fibrosis) should be considered to have HPS unless proven otherwise.

The progression of HPS-PF is characterized by the development of dyspnea and increasingly debilitating hypoxia. Thus, it is recommended that all patients with HPS-1, -2 , and -4 be evaluated for lung function during adolescence and yearly thereafter [328]. Pulmonary physiology is best evaluated with pulmonary function tests including diffusing capacity for carbon monoxide. Early evidence of pulmonary fibrosis might be manifested by a decrease in force vital capacity and lung volumes (indicating a restrictive defect) and reduced diffusing capacity for carbon monoxide, which is typical of fibrosing lung disorders. A sixminute walk test (6MWT) using a portable oximeter should also be considered as hypoxemia may only be detected during exertion in early stages of the disease.

HPS-PF is best evaluated by high-resolution computed tomography. Common changes include ground-glass opacities, reticulation of interstitial spaces and, with advanced disease, loss of lung volume, honey-combing, and traction bronchiectasis [314, 354, 355]. The predominant findings in HPS-PF occur within the lung periphery and progress towards the central portion of the lung as the disease progresses. The most common abnormalities in cases of minimal severity 
are interlobular septal thickening, reticular opacities, ground-glass opacities and subpleural honey-combing. As the disease progresses, reticular opacities and subpleural honeycombing are present to a greater extent. Additionally, a wider range of findings including bronchiectasis, peribronchovascular thickening, and pleural thickening have been reported. In severe cases, sub-pleural honeycombing and bronchiectasis are more common, and subpleural reticular opacities, groundglass opacities, and peribronchovascular thickening are also present $[322,354$, 356]. Thus, although the pattern of pulmonary fibrosis in HPS-PF may be similar to that observed in IPF, several findings are not consistent such as ground glass opacification and pleural thickening (Figure 49).

In contrast to cases being evaluated for IPF and related disorders, a lung biopsy is not recommended in suspected HPS-PF due to the bleeding diathesis. Imaging studies and pulmonary function tests could be conducted to track progression of fibrosis. Bronchoscopy has been used for research purposes, but does not have proven benefits for HPS diagnosis. In short, the diagnosis of HPSPF differs from that of IPF in that lung biopsy is not recommended, and a high level of suspicion is required at an early age in patients with HPS-1, -2 and -4 .

\subsection{Treatment and Management of HPS-PF}

At the moment, no Federal Drug Agency (FDA) approved interventions are specifically designated for the treatment of HPS and HPS-PF, and progress in this 

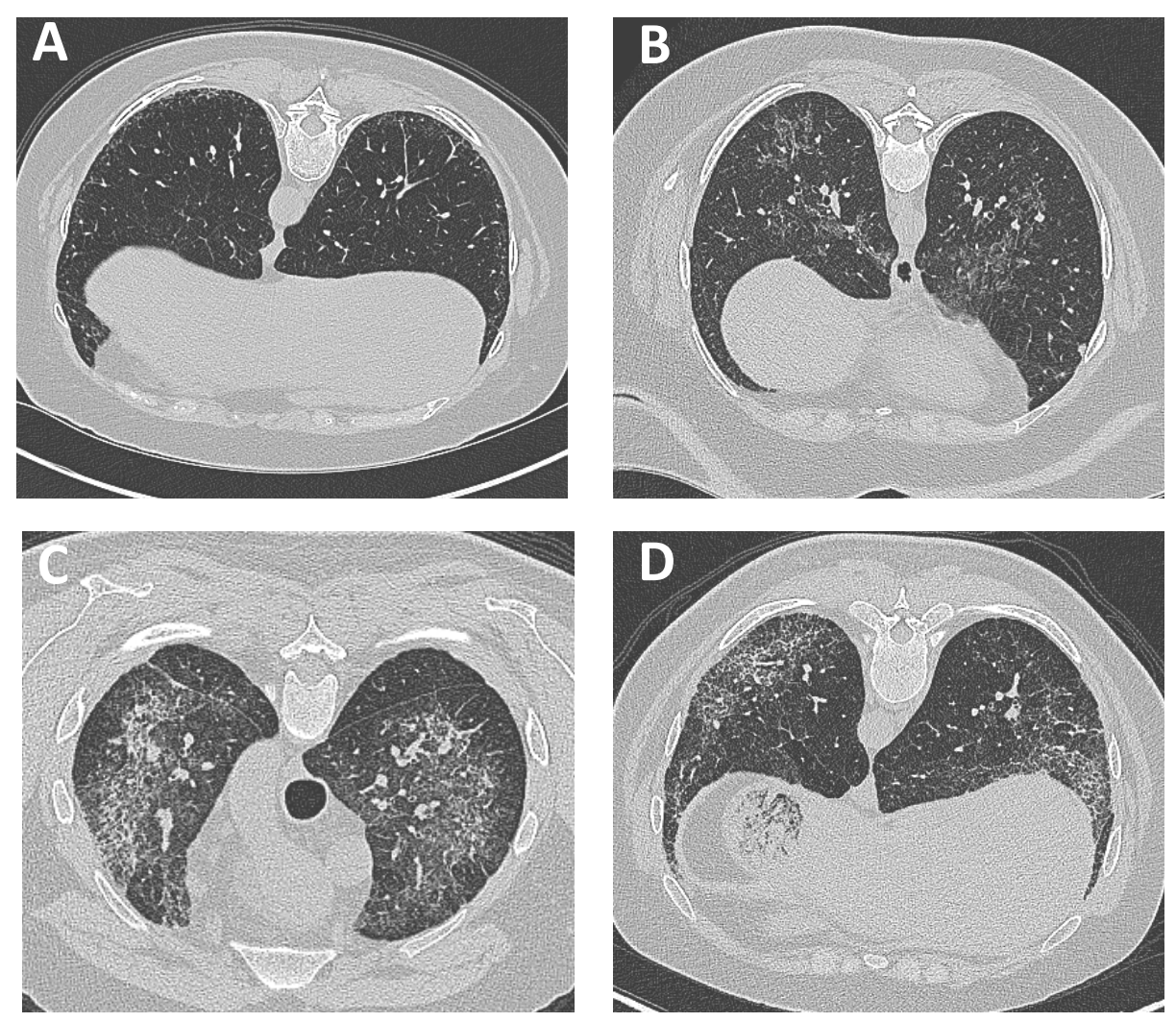

Figure 49. Examples of Chest CT findings in Subjects with HPS-PF

Vicary et. Al., 2016

(A) Case of 50 years old patient with HPS-1 showing mild changes consistent of subpleural infiltrates. (B) Case of 41 years old patient with central infiltrates. (C) Ground glass opacification. (D) Case of 31 years old patient with mid to upper lung infiltrates (D). The images used were from patients consented under the Rare Lung Diseases Consortium HPS study under Vanderbilt University IRB protocol number 150104. 
area has been hindered by the lack of large placebo-controlled, randomized clinical trials. Similar to IPF, corticosteroids have not shown clinical efficacy and are not recommended for HPS-PF. Thus, until safe and effective treatment strategies are developed, lung transplantation may remain the only effective treatment $[319,354$, 357].

As is the case for IPF, treatment relies on preventive care and support as needed. Patients with hypoxemia should be provided supplemental oxygen. Infection prophylaxis is also critical; thus, influenza and 13-valent and 23-valent pneumococcal vaccinations are recommended, and administered with caution to avoid intramuscular bleeding. Additionally, a 5 year polysaccharide booster vaccine may be beneficial [358]. Avoidance of cigarette smoke and other pulmonary irritants is critical, while daily exercise is recommended to avoid deconditioning. HPS patients should avoid unprotected eye and skin exposure to the sun due to their albinism. Menometrorrhagia is common in women, but can be lessened with medication [315, 328]. Further, patients with HPS should avoid aspirin and non-steroidal anti-inflammatory drugs due to platelet inhibition. Extensive injuries should prompt a hospital visit and consultation of a hematologist.

The approval of two new anti-fibrotic drugs, pirfenidone and Nintedanib, for the treatment of IPF, has prompted new interest in identifying drugs capable of reversing or, at the very least, halting the progression of HPS-PF. It should be emphasized that these drugs are not curative, but instead appear to slow down the decline of lung fibrosis in IPF. Pirfenidone, a small molecule capable of inhibiting TGF- $\beta$, showed success in slowing pulmonary dysfunction in clinical trials with IPF 
patients [50, 52]. Interestingly, ex vivo, pirfenidone showed significantly decreased inflammatory cytokines in HPS patient alveolar macrophages [330]. An initial clinical trial of HPS-1 patients with pirfenidone showed no significant slowing of fibrosis progression by forced vital capacity (FVC) [329]. Secondary analysis of this patient population showed patients with an initial FVC value $>50 \%$ of expected had significant decrease in the progression of their pulmonary fibrosis. Disappointingly, a follow-up study with HPS-1 and -4 patients with milder disease showed no significant benefit and was stopped prematurely, but it was likely underpowered [359]. Future clinical studies will be needed in HPS to identify effective treatments.

Considering the genetic basis for the development of HPS, one would predict that gene transfer might be successful in treating this disease. In fact, others have used lentiviruses for the transfer of the HPS1 gene into human dermal melanocytes in vitro and shown correction of the expression and function of the HPS1 gene in these cells [325]. However, difficulties in gene transfer methods in the lung, particularly to the alveolar epithelium, hamper progress in this area, and much still needs to be learned before gene therapy is considered for potential correction of BLOC-3 dysfunction.

\subsection{Summary and Discussion}

HPS is an autosomal recessive disorder caused by defects in the biogenesis of lysosome-related organelles (LROs) and intracellular trafficking. 
Even though HPS is considered an orphan disease (affecting less than 200,000 individuals in the U.S.), its consequences are devastating and often affect young individuals. The epidemiology, natural history, clinical heterogeneity, and best approach to pulmonary monitoring in this population remain insufficiently understood. Despite significant scientific advances, additional research is needed to identify the mechanisms by which the genetic mutations responsible for HPS result in the clinical manifestations of the syndrome. In vitro and ex vivo studies with tissue, cells, blood, bronchoalveolar lavage fluid and other samples collected from patients, as well as in vivo studies in animal models hold promise for determining relevant biological pathways for intervention. Well-conducted placebo controlled and randomized clinical trials will accelerate the evaluation of new antifibrotic agents and other interventions with therapeutic potential. Undoubtedly, the small number of patients affected by HPS will make large-scale studies difficult. Currently, two clinical trials are recruiting HPS-PF patients to hopefully develop better biomarkers for disease progression (NCT02368340, NCT00001456). In the end, such investigations should lead to effective and safe therapies for HPS and its manifestations, especially pulmonary fibrosis. In the meantime, we must find ways to address the clinical needs of these patients. The diagnosis, management, and follow-up of these patients will require the establishment of multidisciplinary centers of excellence staffed with experts in this area. In Puerto Rico, the increased incidence of HPS warrants the development of such a center. Considering that centers with capabilities for lung transplantation are not available in Puerto Rico, connections with centers in the U.S. with transplantation capability 
is important since this procedure is currently the only option for a significant number of patients with HPS-PF. 


\section{CHAPTER VIII}

\section{SUMMARY AND DISCUSSION}

Pulmonary fibrosis affects millions worldwide. This condition is associated with excessive accumulation of activated fibroblasts and chaotic deposition of collagens and other extracellular matrices that lead to obliteration of the healthy lung structure. In many cases, this condition is progressive, thereby leading to lung dysfunction and, ultimately, respiratory failure and death. This is true for IPF, a stereotypical fibrosing lung disorder that affects over 100,000 patients in the U.S., and carries a poor prognosis, with an average survival limited to 3 years after diagnosis [1, 2]. Although two FDA-approved drugs have recently reached the market, they do not improve lung function and long term efficacy is uncertain [50, $52,54]$. Thus, studies directed at better understanding the pathophysiology of this condition and its early detection are needed.

Exactly how tobacco contributes to this condition is unknown. Understanding the pathogenesis of tobacco-related diseases is a significant public health problem, with approximately 40 million Americans smoking cigarettes. Tobacco smoke is thought to contribute to the development and progression of pulmonary fibrosis as many patients with IPF and related fibrosing lung disorders 
are current or ex-smokers [1, 23-25]. This research focused on a major component of tobacco, nicotine, because of recent studies showing that, in addition to promoting addiction, nicotine may stimulate cell proliferation, affect redox reactions, influence inflammation, and promote tissue remodeling during embryogenesis. Thus, this dissertation set out to study if nicotine promotes ECM expression in pulmonary fibroblasts, the main effector cell in pulmonary fibrosis, and the mechanisms responsible for these effects; examine the impact of nicotine on injury-induced lung fibrosis; and determine if nicotine influences survival after injury. We also set out to test the prognostic impact of non-invasive measures capable of detecting early progression of fibrosis. Multiple in vitro and in vivo models as well as national databases were used to address these complex areas.

We found that nicotine promotes the expression of fibronectin and type I collagen, ECM components highly expressed in injured tissues and implicated in wound healing and disrepair. Interestingly, we identified $\alpha 7 \mathrm{nAChRs}$ as the receptors responsible for these events. Based on the above, we predicted that nicotine would enhance fibrosis in a bleomycin-model of lung injury. Nicotine did enhance fibrosis in this model but, interestingly, it appeared to be both harmful and protective as higher doses of bleomycin increased mortality, yet nicotine reduced this harmful effect. The latter was mediated via $\alpha 7 \mathrm{nAChRs}$ as well. Finally, we defined a new formula obtained through lung physiological testing that may be useful in the clinical arena for early detection of progression of lung fibrosis. These findings are considered novel and important for the following reasons. 
First, our data provide a mechanistic description that links tobacco exposure to the development of fibrosing lung disease. However, it is important to note that nicotine does not cause lung fibrosis. Instead, it promotes relative alterations in the composition of the ECM without altering the overall tissue architecture. This effect, termed transitional remodeling, may not create physiological abnormalities at baseline, but we speculate that it may render the host susceptible to disrepair after a second insult (e.g., infection or inhalation of toxicants). This is an intriguing concept as it suggests that other conditions might promote transitional remodeling. In fact, it has been reported that chronic alcohol abuse might lead to similar changes [360]. Of note, aging is also associated with transitional remodeling. This is highly relevant since aging is yet another factor linked to the development of IPF [361]. Thus, identifying challenges like nicotine, that can promote transitional remodeling would be important as these observations might allow us to identify those which are susceptible to pulmonary fibrosis after injury. Of course, genetics, extent of injury, nature of the insult, and other factors are likely to contribute, and this explains why not all smokers develop pulmonary fibrosis.

The concept of transitional remodeling is also important because it raises questions as to how this process may promote disrepair. Our work shows that monocytic cells cultured atop of ECMs derived from nicotine-treated lung fibroblasts express high levels of the pro-inflammatory cytokine IL-1 $\beta$. Thus, we speculate that chronic nicotine exposure might promote transitional remodeling that does not cause disease at baseline, but may promote disrepair after injury by 
stimulating excessive inflammation induced through its interaction with immune cells recruited to the lung after a second insult (Figure 4).

Exposure to tobacco smoke is historically pro-inflammatory, exposing the lung to thousands of compounds and heat. Conversely, nicotine alone is typically considered anti-inflammatory with activation of the cholinergic anti-inflammatory pathway [362]. This belief is conflicted by others demonstrating nicotine can contribute to disease by stimulation of inflammation [363]. Together, the role of nicotine is still being investigated, but the differential effects of nicotine on inflammation may depend on dosage, organ and cells exposed. This is supported by the presence of $\alpha 7$ nAChRs present on several different cell types present throughout the body. Thus, nicotine may directly reduce inflammatory processes in immune cells, but the production of transitional matrices indirectly induces inflammation. At baseline, the anti-inflammatory effects of nicotine might be helpful to avoid disrepair, but this might render the host susceptible to infection. On the other hand, nicotine's pro-inflammatory effects might be helpful to combat infection, while promote disrepair and tissue fibrosis. Fine tuning of these processes will be required to target cholinergic signaling in ways that promote healthy rather than a fibrotic lung state.

This work also suggests that the newly deposited transitional matrix may affect other pulmonary cells involved in the reparative process such as epithelial cells, lung fibroblasts, and endothelial cells. We speculate that by altering the underlying ECM, the injured lung is less able to return to its original structure after injury. If true, preventing the development of a transitional matrix might be 
therapeutic. On the other hand, it is conceivable that transitional matrices are necessary for adequate repair after certain (and perhaps less devastating) insults. Thus, further investigations are needed to determine the true role of transitional matrices prior to engaging in work directed at targeting this process in the clinical arena. Nevertheless, our work provides a model and a framework on which to design such studies.

Additionally, an important aspect of the concept of transitional remodeling is whether it affects the lung at baseline in ways that promote disrepair other than via inflammation. We hypothesized that perhaps nicotine-induced transitional remodeling may affect tissue stiffness. The latter is important when considered against emerging literature suggesting a role for mechanobiology in the control of biological functions. In the lung, one can envision cells embedded in a normal ECM with a certain stiffness that allows for careful regulation of cellular signaling. This is disrupted in fibrosis where tissue stiffness is greatly enhanced, thereby promoting differentiation, fibroproliferation and further ECM overexpression. However, we do not know if nicotine-induced transitional remodeling influences tissue stiffness. We predict that nicotine-induced transitional remodeling may only enhance tissue stiffness slightly, but by promoting disrepair and enhanced fibrosis, it might lead to significant alterations in tissue stiffness after a second insult. We set out to study this using atomic force microscopy and found increases in lung tissue stiffness in animals exposed to both bleomycin and nicotine when compared to those treated with bleomycin alone. These preliminary data needed to be 
confirmed and further investigations will be required to determine the true impact of transitional remodeling on lung tissue stiffness.

Another important observation made by this work relates to $\alpha 7 \mathrm{nAChRs}$, as these receptors mediated the effects of nicotine in our in vitro and in vivo models. This is important for two main reasons. First, there are at least $13 \mathrm{nAChRs}$ that could be targeted, but our studies point to $\alpha 7 \mathrm{nAChRs,} \mathrm{thereby} \mathrm{accelerating} \mathrm{this}$ research significantly. Also important is the fact that there are currently clinically available FDA-approved agents capable of targeting these receptors (e.g., Varenicline) and others in development (PNU-282987). Therefore, technology is already available to develop safe and effective methods of targeting these receptors if further data confirm that $\alpha 7 \mathrm{nAChRs}$ are indeed worth targeting for intervention in fibrosing lung disorders. However, it should be highlighted that $\alpha 7$ nAChRs are present in nearly all cells, ranging from neuronal cells to pulmonary cells. Thus, targeting this receptor might create problems as other organs are affected (e.g., brain). Also, how $\alpha 7$ nAChRs modulate intracellular signaling in the presence of other nAChRs is not entirely clear. Additionally, our data suggest that absence of $\alpha 7 \mathrm{nAChRs}$ has unforeseen effects at homeostasis. One explanation is that its elimination might unveil the roles of other nAChRs which may or may not be protective in the setting of injury and disrepair. The cholinergic signaling system is quite complex and further information will be required before these findings could be exploited at the clinic.

Despite the above, our studies strongly suggest that chronic nicotine exposure promotes lung tissue remodeling and may enhance inflammation, 
thereby promoting disrepair after injury and fibrosis. Thus, avoidance of tobacco exposure is important. However, we also found that nicotine has some protective effects. In our hands, and as observed by others, higher doses of bleomycin induced mortality. However, nicotine was protective in this setting and this effect was again mediated via $\alpha 7 \mathrm{nAChRs}$. Thus, like a coin, nicotine appears to have two faces. On one hand, nicotine can promote disrepair after injury, whereas on the other hand, it can promote survival. This is important as one would need to dissect these distinct effects to take advantage of the beneficial ones, while reducing the injurious ones. In our model, we found that nicotine was associated with alterations in cytokine expression, but other than significant changes in IL-1 $\beta$, little was found that could explain the observations made. However, we did observe an upregulation of superoxide dismutase expression and activity in the lungs of nicotine-treated animals, and these effects was eliminated in the $\alpha 7$ nAChRs knockout mice. Thus, increased anti-oxidant activity through expression of SOD might explain the beneficial effects of nicotine on injury-induced death, but this will require further investigation. Even more convoluting is the fact that recent data suggest that anti-oxidants might benefit some patients with IPF, while not having an effect and even worsening outcomes in many others [364, 365]. These effects are related to genetic variants that influence responsiveness to these agents (e.g., TOLLIP) [366]. Our data suggests that in patients with such genetic variants, nicotine might be protective, while it might be detrimental in others. Further research linking these events to genetic variants will be required before we can determine the true role of nicotine in fibrosing lung disorders and death. 
Together, our studies help explain the role of smoking in fibrosing lung disorders, unveil $\alpha 7 \mathrm{nAChRs}$ as potential targets for intervention, further define the concept of transitional remodeling, its impact, and reveal how chronic nicotine exposure might influence multiple systems in ways that promote tissue remodeling. However, this work would not be useful if this new knowledge cannot be translated to the clinical arena. To that end, we began to examine ways to detect early progression of fibrosis. For this, we moved from bench work and animal models to human data. This retrospective analysis revealed that a new formula based on variables obtained via regular physiological testing might detect early progression of fibrosis. This is important for clinical research as many studies evaluating the impact of novel interventions in lung fibrosis require a significant number of patients and at least 52 weeks. This explains why these studies typically require a multi-center approach and tens of millions of dollars for completion. However, novel approaches capable of identifying disease progression earlier would potentially decrease the number of subjects required as well as the time needed to reach a statistically meaningful result. Based on our data, we speculate that the IMPULSIS and CAPACITY studies, which helped bring the two new anti-fibrotic drugs to the market, could have been terminated much earlier, perhaps 3-6 months earlier, thereby decreasing expenses by at least a third. More importantly, this approach would be extremely helpful at the clinic as clinicians could identify patients not responding to a particular intervention earlier without the need for radiologic testing and larger waiting periods. 
One interesting aspect of the new formula is that it predicted early progression based on the drop of physiological function, but it failed to predict hospitalization and acute exacerbations. This suggests that deterioration of lung function is not directly correlated to those other variables of patient outcomes. Based on these observations, this new formula will need to be tested for several variables prospectively to determine its true sensitivity and specificity.

In summary, this work provides a foundation for further research directed at defining the true role of tobacco and how interventions targeting the cholinergic system might impact fibrosing lung disorders in the clinic (Figure 50). 


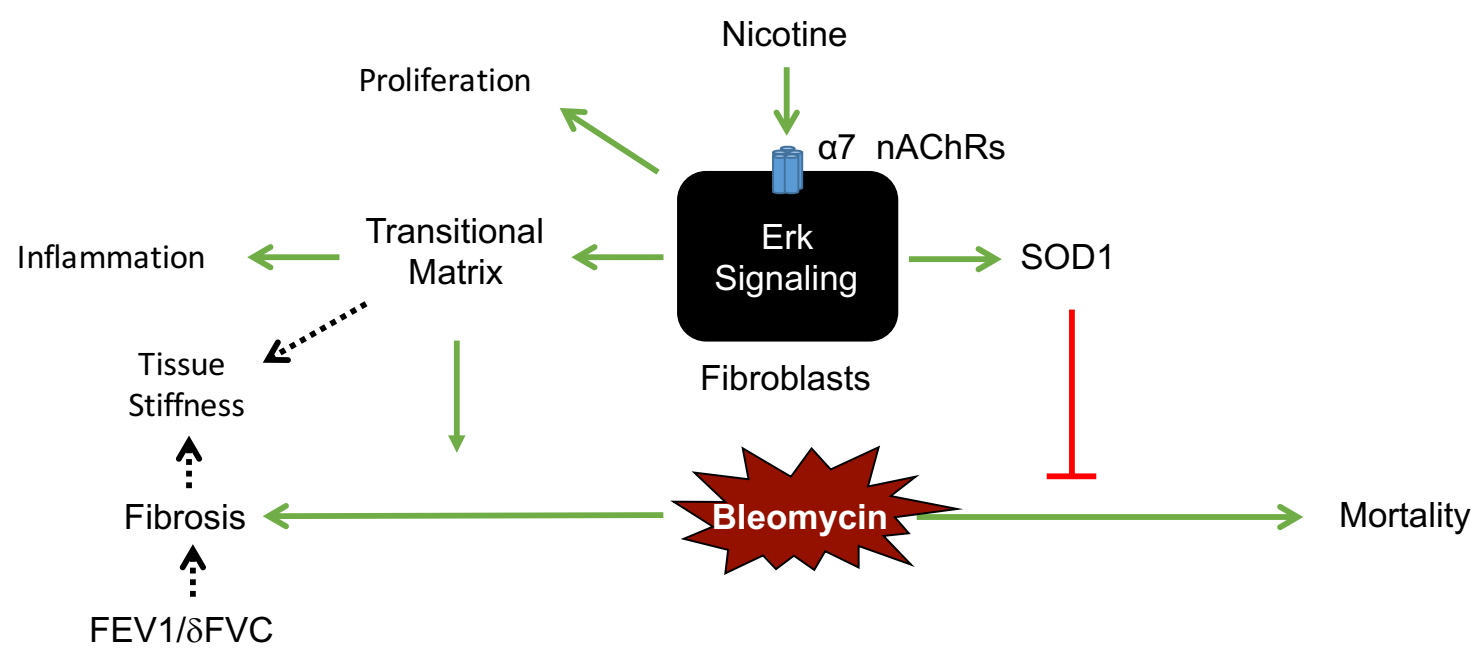

\section{Figure 50. Dissertation Summary}

Nicotine induces a transitional matrix consisting of increased collagen type I, fibronectin, and $\alpha$-SMA expression through $\alpha 7$ nAChRs. This transitional matrix renders a host susceptible to accelerated pulmonary fibrosis exposed to lung injury. Alternatively, nicotine induces the anti-oxidant, SOD 1, which appear to protect against mortality induced by bleomycin-induced lung injury. The promotion of both remodeling and anti-oxidants is an intriguing idea, because it suggests that activation of $\mathrm{nAChR}$ might decrease oxidative stress, while increasing tissue remodeling. This interaction may be critical to the pathogenesis of chronic lung disorders like pulmonary fibrosis characterized by ECM remodeling with low levels of inflammation. Lastly, including a measure of obstructive airway in the

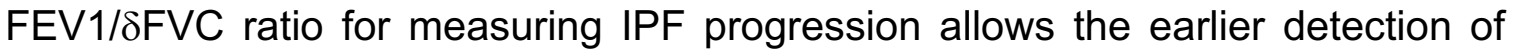
idiopathic pulmonary fibrosis progression. 


\section{STRENGTHS AND WEAKNESSES}

\section{Strengths}

There were several strengths of this dissertation. One strength is the identification of a transitional matrix as a new potential mechanism mediating interaction between pulmonary fibrosis and environmental toxicants (i.e., nicotine). This work describes a characterization of one in vitro and two murine models of lung injury (acute lung injury and pulmonary fibrosis) after chronic nicotine exposure. Characterization of these models using $\alpha 7 \mathrm{nAChR}$ knockout cells and mice allows for a better understanding of how nicotine- $\alpha 7 \mathrm{nAChR}$ interaction affects the lung prior to and after exposure to a secondary injury. The findings of this study provide rationale to investigate $\alpha 7 \mathrm{nAChR}$ as a therapeutic target for clinical treatment, especially with drugs currently in development and FDA approved (e.g. varenicline and PNU-282987).

Additionally, development and verification of lung decellularization methodology allows for the investigation of the mechanical properties and integrin interactions of the lung. Pulmonary fibrosis is marked by pulmonary dysfunction, which can be further investigated using acellular lungs. Understanding differences in extracellular matrix composition induced by a pulmonary challenge can provide new therapeutic targets separate from cells.

Lastly, clinically tracking the progression of pulmonary fibrosis is critical in patient care and clinical trials. Developing a new non-invasive measure of fibrosis 
progression allows for improved patient care and shorter clinical trials, save development costs and accelerated timeline to drugs to receive FDA approval.

\section{Weaknesses}

Although several models and approaches were used in this dissertation, it should be emphasized that most of the studies were based on in vitro and animal models of lung disease. The model of bleomycin-induced fibrosis is the most commonly used murine model to investigate pulmonary fibrosis, but it does not fully capitulate the human disease. The model does not reflect the idiopathic nature of IPF and given time, bleomycin-induced fibrosis will naturally resolve.

Furthermore, this dissertation focuses on nicotine, one of several thousand compounds in cigarette smoke. Utilizing nicotine alone, allowed investigation of specific cellular mechanisms and pathways, but lacks the interactions seen in the complex chemical composition of cigarette smoke. Additionally, nicotine was administered via the oral route rather than via inhalation. When inhaled, nicotine concentrates in the lung before distributing through the bloodstream. This characteristic was not mimicked by the oral administration used in this dissertation. Thus, it is not certain if our findings are generalizable to models of smoke inhalation or if they are relevant to humans. Currently, lungs-on-a-chip systems are being developed, which will allow for better investigations of the effects of tobacco smoke on pulmonary cells [367]. In contrast, they might be quite important considering the emergence of e-cigarettes in younger adults. 
Lastly, despite showing that FEV1/8FVC detected earlier pulmonary decline when compared to FVC alone, specificity still needs to be verified. This will involve utilizing CT scans to verify patient loss of $\mathrm{FEV} 1 / \delta \mathrm{FVC}$ is associated with increased pulmonary fibrosis. This work may end up requiring an increase or decrease in the $10 \%$ threshold to be prognostically accurate.

The different approaches used in this dissertation provide a variety of weaknesses, but together, these can be addressed through future research and utilizing new methods; some of which are addressed in my future directions. 


\section{FUTURE DIRECTIONS}

\section{Pathway of Nicotine Protection}

Although this dissertation shows the involvement of $\alpha 7 \mathrm{nAChRs}$ and Erk, the pathways by which nicotine induces collagen type I and fibronectin are not completely elucidated. Whether the same pathway also controls the nicotine induction of SOD1 is unknown. Additionally, the potential involvement of nicotine as well as SOD2 and SOD3 need to be investigated. Finding additional pathway mediators will be important, including potentially, Nrf2. Knockout and overexpressing mice for the three SODs are available, providing useful tools to further investigate the role of SODs in acute lung injury.

\section{Integrin Induction of IL-1 $\beta$}

Showing a transitional matrix can induce IL-1 $\beta$ expression through $\alpha 2$ integrin activation is intriguing concept. Investigating this mechanism of inflammation activation could provide druggable targets. Excessive inflammasome activation has been associated with neurogenerative and metabolic diseases, but recent research has also implicated inflammasomes in pulmonary fibrosis [368, 369]. Inflammasome activation drives cleavage of $\mathrm{IL}-1 \beta$ to its active, proinflammatory, form. Some research has implicated integrins in the activation with inflammation. In a murine model of arthritis, blockade of $\alpha 2 \beta 1$ integrin decreased 
inflammation and the number of Th17 cells [370, 371]. Additionally, another murine model of rheumatoid arthritis, knockout of $\alpha 2 \beta 1$ integrin reduced basal levels and blocked TNF- $\alpha$ induction of ERK-1/2 [372]. In airway smooth muscle cells, $\alpha 2 \beta 1$ integrin activation mediated IL-1 $\beta$-dependent secretory responses [373]. Lastly, $\alpha 2 \beta 1$ is not the only integrin worth investigation, with inhibition of the $\alpha 5 \beta 1$ integrin inhibiting NLRP3 expression and IL-1 $\beta$ expression and secretion THP cells [374].

\section{Anti-Oxidant Induction}

Another form of oxidant stress is best known as redox stress and can be measured by evaluating redox potential [375]. Redox stress is created by the relative concentrations of thiol disulfide couples such as cysteine and cysteine (Cys/CySS) and glutathione and glutathione disulfide (GSH and GSSG). Alterations in these thiol disulfide couples result in alterations in redox potential (Eh). Eh Cys/CySS exerts its main effects extracellularly, while Eh GSH/GSSH is more relevant intracellularly [376]. nAChRs could also play important roles in oxidant stress as the Roman lab recently reporting that $\alpha 4 \mathrm{nAChRs}$ might serve as sensors of Eh Cys/CySS in lung fibroblasts [377]. Additionally, our group has also reported that bleomycin-induced lung injury is associated with oxidation of the Eh Cys/CySS, which stimulates the proliferation of primary lung fibroblasts and their expression of $\alpha$-smooth muscle actin and fibronectin, which are also hallmarks of IPF [378-381]. 


\section{Elucidating Complicated Tobacco Smoke}

Having evaluated the role of $\alpha 7 \mathrm{nAChRs}$ in mediating the effects of nicotine, we have also explored the effects of cigarette smoke extract (CSE) on lung fibroblasts. As expected, CSE stimulated significant fibronectin protein production at 6 months (Figure 51 and Figure 52). With the myriad of chemicals in tobacco, it makes it difficult to elucidate the vast number of changes made by tobacco exposure, but recently a 'smoking-lung-on-a-chip' system has been developed, which provides a powerful tool to study the effects of whole cigarette and ecigarette smoke on lung cells [382].

\section{Tobacco-related Genetic Changes}

Advances in genetic technology have invigorated the focus of the scientific community on genetic and epigenetic changes driving diseases. The multifarious

composition of tobacco causes a myriad of genetic changes [383]. Our lab is currently studying epigenetic changes caused by nicotine exposure, which could help to uncover genetic changes caused by nicotine. These changes could provide druggable targets with histone acetylators and deacetylators. 


\section{Cancer}

Lung fibrosis has been associated with other diseases, including lung cancer [384]. In the Roman lab, Caleb Greenwell has done some work on the influence of pulmonary microenvironment on lung cancer, showing increased lung metastasis in the presence of pulmonary fibrosis (Figure 53) Additionally, over the course of my Ph.D., I conducted some research on lung cancer, consisting of a published review of the mammalian target of rapamycin (mTOR) in lung cancer and the potential for CRKL to mediate epithelial-mesenchymal transition, an important process in metastasis, in lung cancer (Submitted) [385]. 

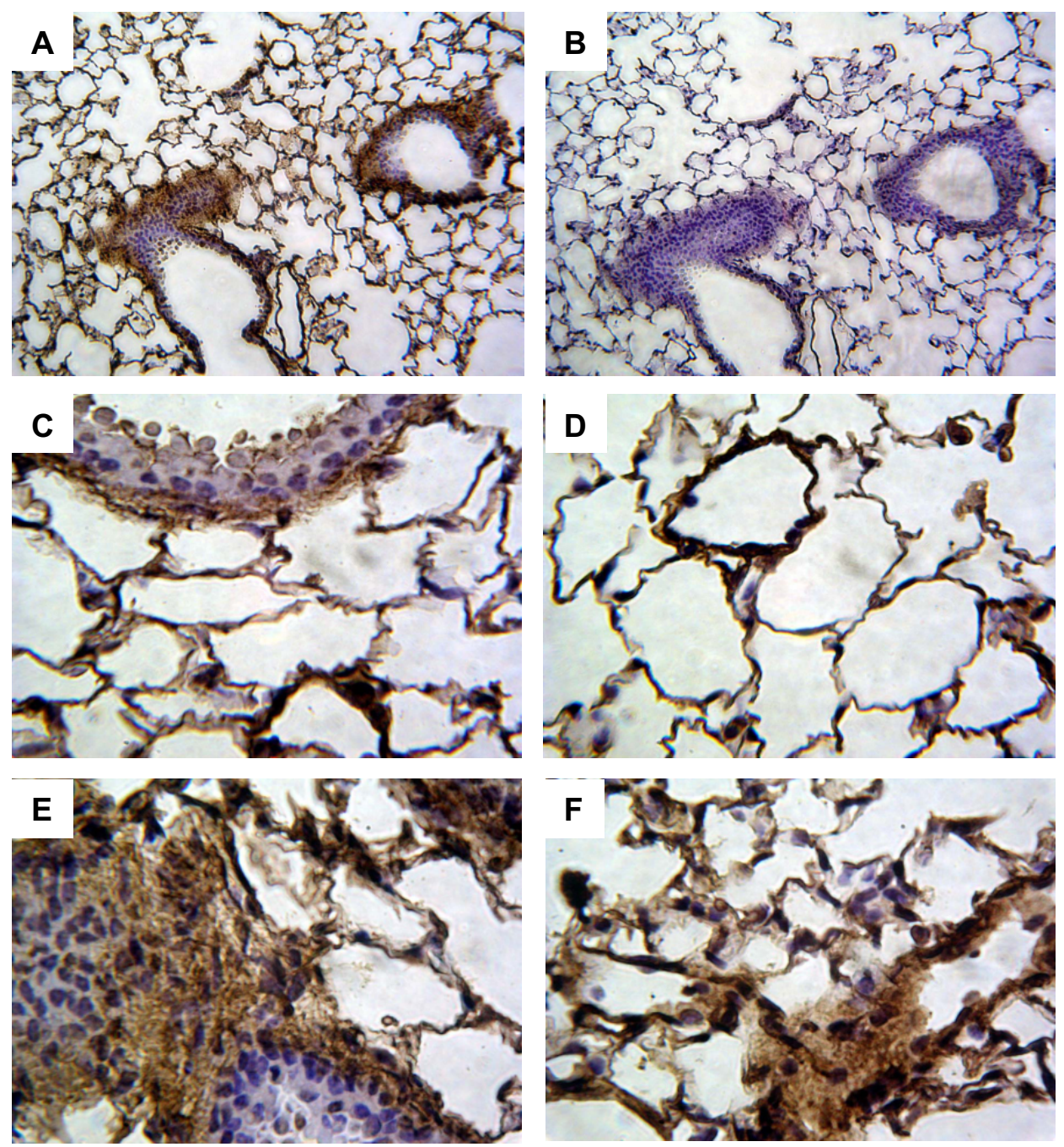

Figure 51. Cigarette Smoke Induces Fibronectin In Vivo

Roman et. Al. Unpublished

(A) In collaboration with Douglas Arenberg at the University of Michigan, mice were exposed to tobacco smoke for up to 6 months (see methods). They were then sacrificed and the lungs processed for immunohistochemistry. A: anti-fibronectin antibody; B: Control IgG; C and D: Control lungs stained for fibronectin. E and F: Fibronectin staining in smoke-exposed animals. 
A

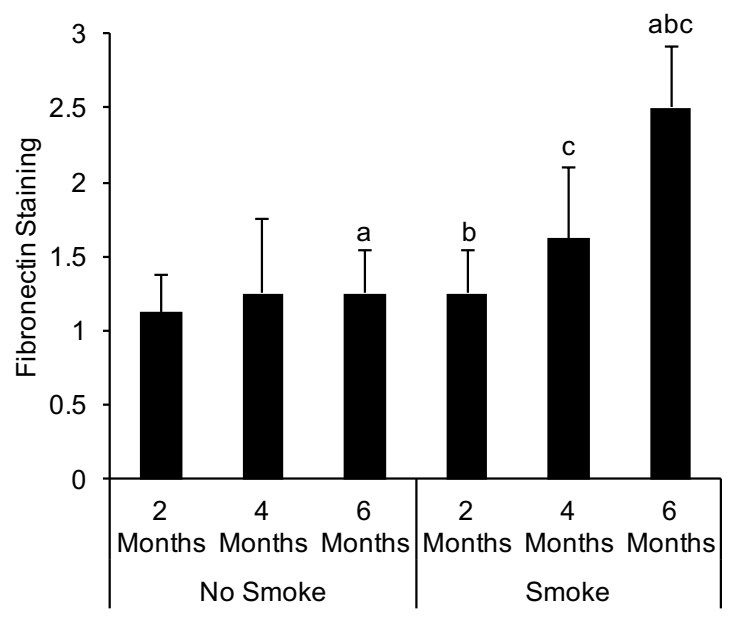

B

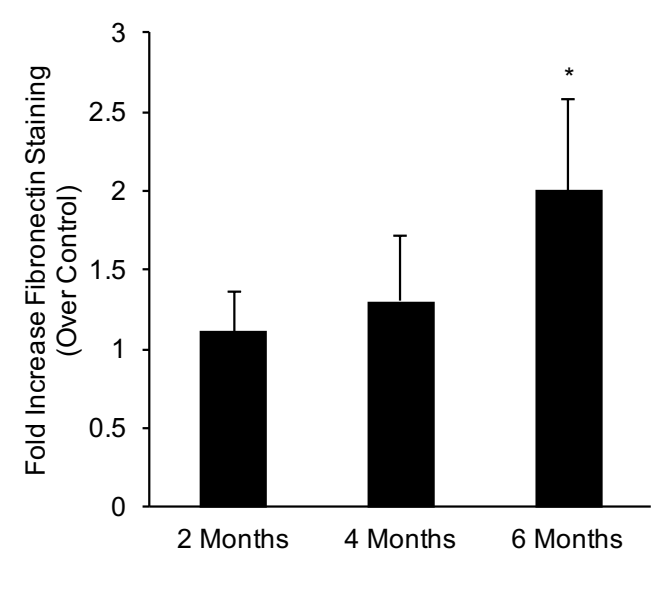

Figure 52. Cigarette Smoke Induces Fibronectin Time-Dependently In Vivo

Roman et. Al. Unpublished

(A) Blinded evaluation of intensity of fibronectin staining at different stages. (B)

Difference in fibronectin staining between control and CSE exposed mice as fold change. Significance was assessed using $p$ values $<0.05$ obtained by two-tailed ttest. 
A

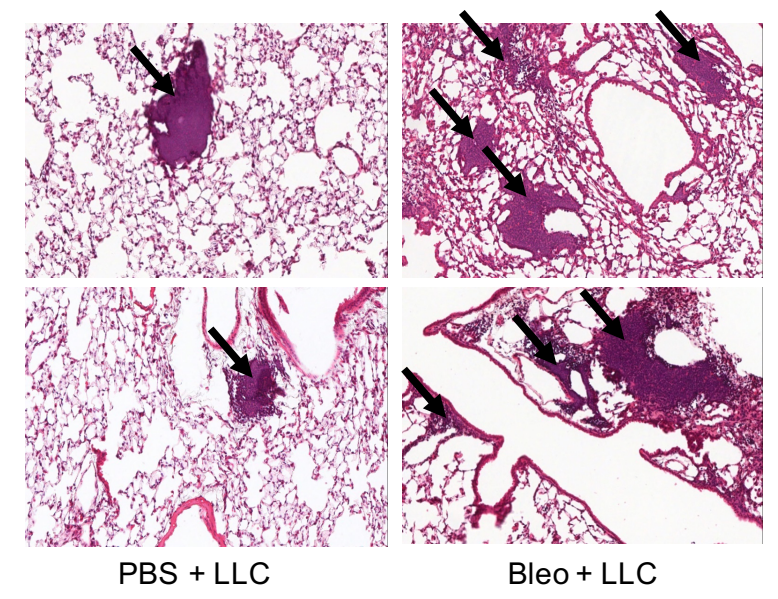

B

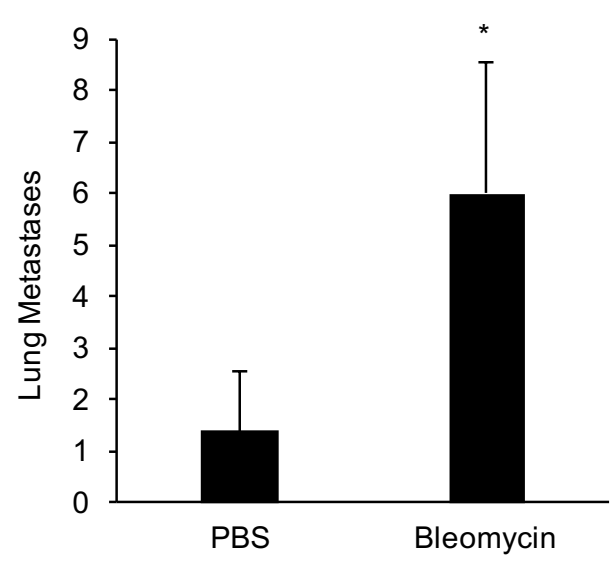

Figure 53. Bleomycin Treatment is Associated with Increased Metastases in Aged Lungs

Greenwell et. Al. Unpublished

(A) Aged ( 9.5 months) mice were treated with PBS or bleomycin followed by injection with Lewis Lung Carcinoma cells. Lungs were inflated at standard pressure, fixed in formalin, paraffin-embedded, and sectioned $(5 \mu \mathrm{m})$ for histological analysis. Representative images depict PBS- and bleomycin-treated aged mice at $14 x$ magnification. Tumor metastases are observed in all images (indicated by arrows), while fibrosis is clear in the bleomycin treated lungs. (B) The number of lung metastasis was analyzed. Bleomycin instillation in aged animals increased the number of lung metastases compared to PBS. Significance was assessed using $p$ values $<0.05$ obtained by two-tailed t-test. 


\section{REFERENCES}

1. Ley, B. and H.R. Collard, Epidemiology of idiopathic pulmonary fibrosis. Clin Epidemiol, 2013. 5: p. 483-92.

2. King, T.E., Jr., et al., Predicting survival in idiopathic pulmonary fibrosis: scoring system and survival model. Am J Respir Crit Care Med, 2001. 164(7): p. 1171-81.

3. Ryu, J.H., T.V. Colby, and T.E. Hartman, Idiopathic pulmonary fibrosis: current concepts. Mayo Clin Proc, 1998. 73(11): p. 1085-101.

4. Michaelson, J.E., S.M. Aguayo, and J. Roman, Idiopathic pulmonary fibrosis: a practical approach for diagnosis and management. Chest, 2000. 118(3): p. 788-94.

5. Raghu, G., et al., An official ATS/ERS/JRS/ALAT statement: idiopathic pulmonary fibrosis: evidence-based guidelines for diagnosis and management. Am J Respir Crit Care Med, 2011. 183(6): p. 788-824.

6. Fernandez Perez, E.R., et al., Incidence, prevalence, and clinical course of idiopathic pulmonary fibrosis: a population-based study. Chest, 2010. 137(1): p. 129-37.

7. Nadrous, H.F., et al., Idiopathic pulmonary fibrosis in patients younger than 50 years. Mayo Clinic Proceedings, 2005. 80(1): p. 37-40. 
8. Selman, M., T.E. King, and A. Pardo, Idiopathic pulmonary fibrosis:

Prevailing and evolving hypotheses about its pathogenesis and implications for therapy. Annals of Internal Medicine, 2001. 134(2): p. 136151.

9. du Bois, R.M., Strategies for treating idiopathic pulmonary fibrosis. Nat Rev Drug Discov, 2010. 9(2): p. 129-40.

10. Olson, A.L., et al., Mortality from pulmonary fibrosis increased in the United States from 1992 to 2003. Am J Respir Crit Care Med, 2007. 176(3): p. 277-84.

11. Lee, A.S., et al., The burden of idiopathic pulmonary fibrosis: an unmet public health need. Respir Med, 2014. 108(7): p. 955-67.

12. Ryu, J.H., et al., Idiopathic pulmonary fibrosis: evolving concepts. Mayo Clin Proc, 2014. 89(8): p. 1130-42.

13. Kropski, J.A., W.E. Lawson, and T.S. Blackwell, Right place, right time: the evolving role of herpesvirus infection as a "second hit" in idiopathic pulmonary fibrosis. Am J Physiol Lung Cell Mol Physiol, 2012. 302(5): p. L441-4.

14. Selman, M., et al., Surfactant protein $A$ and $B$ genetic variants predispose to idiopathic pulmonary fibrosis. Hum Genet, 2003. 113(6): p. 542-50.

15. Thomas, A.Q., et al., Heterozygosity for a surfactant protein C gene mutation associated with usual interstitial pneumonitis and cellular nonspecific interstitial pneumonitis in one kindred. Am J Respir Crit Care Med, 2002. 165(9): p. 1322-8. 
16. Seibold, M.A., et al., A common MUC5B promoter polymorphism and pulmonary fibrosis. N Engl J Med, 2011. 364(16): p. 1503-12.

17. Traila, D., et al., Short telomeres in pulmonary fibrosis: from genetics to clinical significance. Pneumologia, 2015. 64(1): p. 8, 11-3.

18. King, T.E., A. Pardo, and M. Selman, Idiopathic pulmonary fibrosis. The Lancet, 2011. 378(9807): p. 1949-1961.

19. Allam, J.S. and A.H. Limper, Idiopathic pulmonary fibrosis: is it a familial disease? Curr Opin Pulm Med, 2006. 12(5): p. 312-7.

20. Steele, M.P., et al., Clinical and pathologic features of familial interstitial pneumonia. Am J Respir Crit Care Med, 2005. 172(9): p. 1146-52.

21. Lee, H.L., et al., Familial idiopathic pulmonary fibrosis: clinical features and outcome. Chest, 2005. 127(6): p. 2034-41.

22. Wahidi, M.M., et al., Familial pulmonary fibrosis in the United States. Chest, 2002. 121(3 Suppl): p. 30S.

23. Ryu, J.H., et al., Smoking-related interstitial lung diseases: a concise review. Eur Respir J, 2001. 17(1): p. 122-32.

24. American Thoracic Society. Idiopathic pulmonary fibrosis: diagnosis and treatment. International consensus statement. American Thoracic Society (ATS), and the European Respiratory Society (ERS). Am J Respir Crit Care Med, 2000. 161(2 Pt 1): p. 646-64.

25. Baumgartner, K.B., et al., Cigarette smoking: a risk factor for idiopathic pulmonary fibrosis. Am J Respir Crit Care Med, 1997. 155(1): p. 242-8. 
26. Sorensen, L.T., Wound healing and infection in surgery: the pathophysiological impact of smoking, smoking cessation, and nicotine replacement therapy: a systematic review. Ann Surg, 2012. 255(6): $p$. 1069-79.

27. Prevention, C.f.D.C.a., in How Tobacco Smoke Causes Disease: The Biology and Behavioral Basis for Smoking-Attributable Disease: A Report of the Surgeon General. 2010: Atlanta (GA).

28. Brewer, B.G., A.M. Roberts, and P.P. Rowell, Short-term distribution of nicotine in the rat lung. Drug Alcohol Depend, 2004. 75(2): p. 193-8.

29. N.I.H., Nicotine Addiction. National Institute on Drug Abuse Research Report, 1998. 01-4342: p. 8.

30. Matta, S.G., et al., Guidelines on nicotine dose selection for in vivo research. Psychopharmacology (Berl), 2007. 190(3): p. 269-319.

31. Benowitz, N.L., et al., Oral contraceptives induce CYP2A6 activity and accelerate nicotine metabolism. Clinical Pharmacology \& Therapeutics, 2004. 75(2): p. P36-P36.

32. Molander, L., A. Hansson, and E. Lunell, Pharmacokinetics of nicotine in healthy elderly people. Clin Pharmacol Ther, 2001. 69(1): p. 57-65.

33. Cardinale, A., et al., Nicotine: specific role in angiogenesis, proliferation and apoptosis. Crit Rev Toxicol, 2012. 42(1): p. 68-89.

34. Sekhon, H.S., et al., Prenatal nicotine increases pulmonary alpha7 nicotinic receptor expression and alters fetal lung development in monkeys. J Clin Invest, 1999. 103(5): p. 637-47. 
35. Wongtrakool, C., et al., Nicotine alters lung branching morphogenesis through the alpha7 nicotinic acetylcholine receptor. Am J Physiol Lung Cell Mol Physiol, 2007. 293(3): p. L611-8.

36. Gunther, A., et al., Unravelling the progressive pathophysiology of idiopathic pulmonary fibrosis. Eur Respir Rev, 2012. 21(124): p. 152-60.

37. Loomis-King, H., K.R. Flaherty, and B.B. Moore, Pathogenesis, current treatments and future directions for idiopathic pulmonary fibrosis. Curr Opin Pharmacol, 2013. 13(3): p. 377-85.

38. Kropski, J.A., et al., Genetic studies provide clues on the pathogenesis of idiopathic pulmonary fibrosis. Dis Model Mech, 2013. 6(1): p. 9-17.

39. Selman, M. and A. Pardo, Role of epithelial cells in idiopathic pulmonary fibrosis: from innocent targets to serial killers. Proc Am Thorac Soc, 2006. 3(4): p. 364-72.

40. Sakai, N. and A.M. Tager, Fibrosis of two: Epithelial cell-fibroblast interactions in pulmonary fibrosis. Biochim Biophys Acta, 2013. 1832(7): p. $911-21$.

41. American Thoracic, S. and S. European Respiratory, American Thoracic Society/European Respiratory Society International Multidisciplinary Consensus Classification of the Idiopathic Interstitial Pneumonias. This joint statement of the American Thoracic Society (ATS), and the European Respiratory Society (ERS) was adopted by the ATS board of directors, June 2001 and by the ERS Executive Committee, June 2001. Am J Respir Crit Care Med, 2002. 165(2): p. 277-304. 
42. Phan, S.H., Genesis of the myofibroblast in lung injury and fibrosis. Proc Am Thorac Soc, 2012. 9(3): p. 148-52.

43. Kage, $\mathrm{H}$. and Z. Borok, EMT and interstitial lung disease: a mysterious relationship. Curr Opin Pulm Med, 2012. 18(5): p. 517-23.

44. Coward, W.R., G. Saini, and G. Jenkins, The pathogenesis of idiopathic pulmonary fibrosis. Ther Adv Respir Dis, 2010. 4(6): p. 367-88.

45. Roman, J., et al., An official American thoracic society workshop report: comparative pathobiology of fibrosing lung disorders in humans and domestic animals. Ann Am Thorac Soc, 2013. 10(6): p. S224-9.

46. Roman, J., Fibronectin and fibronectin receptors in lung development. Exp Lung Res, 1997. 23(2): p. 147-59.

47. Roman, J., Extracellular matrices in interstitial lung disease. 3 ed. Interstitial Lung Disease, ed. A.M. M Schwarz and T King. 2003, London, England: B. C. Decker, Inc. 20.

48. Noble, P.W., C.E. Barkauskas, and D. Jiang, Pulmonary fibrosis: patterns and perpetrators. J Clin Invest, 2012. 122(8): p. 2756-62.

49. Uhal, B.D., et al., Alveolar epithelial cell death adjacent to underlying myofibroblasts in advanced fibrotic human lung. Am J Physiol, 1998. 275(6 Pt 1): p. L1192-9.

50. King, T.E., Jr., et al., A phase 3 trial of pirfenidone in patients with idiopathic pulmonary fibrosis. N Engl J Med, 2014. 370(22): p. 2083-92.

51. Richeldi, L., et al., Efficacy and safety of nintedanib in idiopathic pulmonary fibrosis. N Engl J Med, 2014. 370(22): p. 2071-82. 
52. Azuma, A., et al., Exploratory analysis of a phase III trial of pirfenidone identifies a subpopulation of patients with idiopathic pulmonary fibrosis as benefiting from treatment. Respir Res, 2011. 12: p. 143.

53. Wuyts, W.A., et al., First Data on Efficacy and Safety of Nintedanib in Patients with Idiopathic Pulmonary Fibrosis and Forced Vital Capacity of $</=50 \%$ of Predicted Value. Lung, 2016.

54. Nair, G.B., et al., Newer developments in idiopathic pulmonary fibrosis in the era of anti-fibrotic medications. Expert Rev Respir Med, 2016. 10(6): p. 699-711.

55. Cox, T.R. and J.T. Erler, Remodeling and homeostasis of the extracellular matrix: implications for fibrotic diseases and cancer. Dis Model Mech, 2011. 4(2): p. 165-78.

56. Frantz, C., K.M. Stewart, and V.M. Weaver, The extracellular matrix at a glance. J Cell Sci, 2010. 123(Pt 24): p. 4195-200.

57. Matthes, S.A., et al., Chapter 20 - Comparative Biology of the Normal Lung Extracellular Matrix A2 - Parent, Richard A, in Comparative Biology of the Normal Lung (Second Edition). 2015, Academic Press: San Diego. p. $387-402$.

58. Naba, A., et al., The matrisome: in silico definition and in vivo characterization by proteomics of normal and tumor extracellular matrices. Mol Cell Proteomics, 2012. 11(4): p. M111 014647. 
59. Booth, A.J., et al., Acellular normal and fibrotic human lung matrices as a culture system for in vitro investigation. Am J Respir Crit Care Med, 2012. 186(9): p. 866-76.

60. Roman, J., Extracellular matrix and lung inflammation. Immunol Res, 1996. 15(2): p. 163-78.

61. Limper, A.H. and J. Roman, Fibronectin. A versatile matrix protein with roles in thoracic development, repair and infection. Chest, 1992. 101(6): p. 1663-73.

62. Roman, J., Fibronectin and Fibronectin Receptors in Lung Development. Experimental lung research, 1997. 23(2): p. 147-159.

63. Parekh, T., et al., Neutrophil chemotaxis in response to TGF-beta isoforms (TGF-beta 1, TGF-beta 2, TGF-beta 3) is mediated by fibronectin. J Immunol, 1994. 152(5): p. 2456-66.

64. Clark, R.A., Potential roles of fibronectin in cutaneous wound repair. Arch Dermatol, 1988. 124(2): p. 201-6.

65. Graves, K.L. and J. Roman, Fibronectin modulates expression of interleukin-1 beta and its receptor antagonist in human mononuclear cells. Am J Physiol, 1996. 271(1 Pt 1): p. L61-9.

66. Pacifici, R., et al., Collagen-induced release of interleukin 1 from human blood mononuclear cells. Potentiation by fibronectin binding to the alpha 5 beta 1 integrin. J Clin Invest, 1992. 89(1): p. 61-7. 
67. Parekh, T., et al., Neutrophil Chemotaxis in Response to Tgf-Beta Isoforms (Tgf-Beta-1, Tgf-Beta-2, Tgf-Beta-3) Is Mediated by Fibronectin. Journal of Immunology, 1994. 152(5): p. 2456-2466.

68. Bitterman, P.B., Role of fibronectin as a growth factor for fibroblasts. The Journal of Cell Biology, 1983. 97(6): p. 1925-1932.

69. Chang, Z.L., et al., Fibronectin cell-binding domain triggered transmembrane signal transduction in human monocytes. J Leukoc Biol, 1993. 53(1): p. 79-85.

70. Sueblinvong, V., et al., Predisposition for disrepair in the aged lung. Am J Med Sci, 2012. 344(1): p. 41-51.

71. Villiger, B., et al., Bronchoalveolar fibronectin in smokers and nonsmokers. Am Rev Respir Dis, 1981. 124(5): p. 652-4.

72. Song, W.D., et al., Fibronectin and hyaluronan in bronchoalveolar lavage fluid from young patients with chronic obstructive pulmonary diseases. Respiration, 1995. 62(3): p. 125-9.

73. Redington, A.E., P. Sime, P.H. Howarth, and S.T. Holgate. , Fibroblasts and the extracellular matrix in asthma. Inflamamtory mechanisms of asthma, ed. S.T.H.a.W.W. Busse. 1998, New York, NT: Marcel Dekker, Inc. 24.

74. Vanacker, N.J., et al., Effect of combining salmeterol and fluticasone on the progression of airway remodeling. Am J Respir Crit Care Med, 2002. 166(8): p. 1128-34. 
75. Nakstad, B., N.P. Boye, and T. Lyberg, Distribution of bronchoalveolar cells and fibronectin levels in bronchoalveolar lavage fluids from patients with lung disorders. Scand J Clin Lab Invest, 1990. 50(6): p. 587-93.

76. Ayad, S., The extracellular matrix factsbook. 2nd ed. Factsbook series. 1998, San Diego: Academic Press. x, 301 p.

77. Roman, J., et al., Nicotine and fibronectin expression in lung fibroblasts: implications for tobacco-related lung tissue remodeling. FASEB J, 2004. 18(12): p. 1436-8.

78. Muro, A.F., et al., An essential role for fibronectin extra type III domain A in pulmonary fibrosis. American journal of respiratory and critical care medicine, 2008. 177(6): p. 638-45.

79. Kummer, W., K.S. Lips, and U. Pfeil, The epithelial cholinergic system of the airways. Histochem Cell Biol, 2008. 130(2): p. 219-34.

80. Improgo, M.R., et al., From smoking to lung cancer: the CHRNA5/A3/B4 connection. Oncogene, 2010. 29(35): p. 4874-84.

81. Koval, L., et al., Differential involvement of alpha4beta2, alpha7 and alpha9alpha10 nicotinic acetylcholine receptors in B lymphocyte activation in vitro. Int J Biochem Cell Biol, 2011. 43(4): p. 516-24.

82. Sato, K.Z., et al., Diversity of $m R N A$ expression for muscarinic acetylcholine receptor subtypes and neuronal nicotinic acetylcholine receptor subunits in human mononuclear leukocytes and leukemic cell lines. Neurosci Lett, 1999. 266(1): p. 17-20. 
83. Benfante, R., et al., Expression of the alpha7 nAChR subunit duplicate form (CHRFAM7A) is down-regulated in the monocytic cell line THP-1 on treatment with LPS. J Neuroimmunol, 2011. 230(1-2): p. 74-84.

84. Lindstrom, J., Nicotinic acetylcholine receptors in health and disease. Mol Neurobiol, 1997. 15(2): p. 193-222.

85. Roman, J., et al., Nicotine and fibronectin expression in lung fibroblasts: implications for tobacco-related lung tissue remodeling. The FASEB journal : official publication of the Federation of American Societies for Experimental Biology, 2004. 18(12): p. 1436-8.

86. Zia, S., et al., Nicotine enhances expression of the alpha 3, alpha 4, alpha 5, and alpha 7 nicotinic receptors modulating calcium metabolism and regulating adhesion and motility of respiratory epithelial cells. Research communications in molecular pathology and pharmacology, 1997. 97(3): p. 243-62.

87. Phan, S.H., The myofibroblast in pulmonary fibrosis. Chest, 2002. 122(6 Suppl): p. 286S-289S.

88. Sekhon, H.S., et al., Prenatal nicotine increases pulmonary alpha7 nicotinic receptor expression and alters fetal lung development in monkeys. The Journal of Clinical Investigation, 1999. 103(5): p. 637-47.

89. Sekhon, H.S., et al., Prenatal nicotine exposure alters pulmonary function in newborn rhesus monkeys. Am J Respir Crit Care Med, 2001. 164(6): p. 989-94. 
90. Sekhon, H.S., et al., Maternal nicotine exposure upregulates collagen gene expression in fetal monkey lung. Association with alpha7 nicotinic acetylcholine receptors. Am J Respir Cell Mol Biol, 2002. 26(1): p. 31-41.

91. Sekhon, H.S., et al., Prenatal nicotine exposure increases connective tissue expression in foetal monkey pulmonary vessels. Eur Respir J, 2004. 23(6): p. 906-15.

92. Zheng, Y., et al., Nicotine stimulates human lung cancer cell growth by inducing fibronectin expression. Am J Respir Cell Mol Biol, 2007. 37(6): p. 681-90.

93. Wang, Y., et al., Human bronchial epithelial and endothelial cells express alpha7 nicotinic acetylcholine receptors. Mol Pharmacol, 2001. 60(6): p. 1201-9.

94. Lam, D.C., et al., Expression of nicotinic acetylcholine receptor subunit genes in non-small-cell lung cancer reveals differences between smokers and nonsmokers. Cancer Res, 2007. 67(10): p. 4638-47.

95. Moodley, Y.P., et al., Fibroblasts isolated from normal lungs and those with idiopathic pulmonary fibrosis differ in interleukin-6/gp130-mediated cell signaling and proliferation. Am J Pathol, 2003. 163(1): p. 345-54.

96. Yoshida, K., et al., MAP kinase activation and apoptosis in lung tissues from patients with idiopathic pulmonary fibrosis. J Pathol, 2002. 198(3): $p$. 388-96.

97. Robledo, R. and B. Mossman, Cellular and molecular mechanisms of asbestos-induced fibrosis. J Cell Physiol, 1999. 180(2): p. 158-66. 
98. Sullivan, D.E., et al., Tumor necrosis factor-alpha induces transforming growth factor-beta1 expression in lung fibroblasts through the extracellular signal-regulated kinase pathway. Am J Respir Cell Mol Biol, 2005. 32(4): p. $342-9$.

99. Madala, S.K., et al., MEK-ERK pathway modulation ameliorates pulmonary fibrosis associated with epidermal growth factor receptor activation. Am J Respir Cell Mol Biol, 2012. 46(3): p. 380-8.

100. Galuppo, M., et al., MEK inhibition suppresses the development of lung fibrosis in the bleomycin model. Naunyn Schmiedebergs Arch Pharmacol, 2011. 384(1): p. 21-37.

101. Ulloa, L. and K.J. Tracey, The "cytokine profile": a code for sepsis. Trends Mol Med, 2005. 11(2): p. 56-63.

102. Miyazawa, A., Y. Fujiyoshi, and N. Unwin, Structure and gating mechanism of the acetylcholine receptor pore. Nature, 2003. 423(6943): p. 949-55.

103. Borovikova, L.V., et al., Vagus nerve stimulation attenuates the systemic inflammatory response to endotoxin. Nature, 2000. 405(6785): p. 458-62.

104. Matsunaga, K., et al., Involvement of nicotinic acetylcholine receptors in suppression of antimicrobial activity and cytokine responses of alveolar macrophages to Legionella pneumophila infection by nicotine. J Immunol, 2001. 167(11): p. 6518-24.

105. Fujii, T., et al., Constitutive expression of mRNA for the same choline acetyltransferase as that in the nervous system, an acetylcholine- 
synthesizing enzyme, in human leukemic T-cell lines. Neuroscience Letters, 1999. 259(2): p. 71-74.

106. Sato, K.Z., et al., Diversity of $m R N A$ expression for muscarinic acetylcholine receptor subtypes and neuronal nicotinic acetylcholine receptor subunits in human mononuclear leukocytes and leukemic cell lines. Neuroscience Letters, 1999. 266(1): p. 17-20.

107. Gahring, L.C. and S.W. Rogers, Neuronal nicotinic acetylcholine receptor expression and function on nonneuronal cells. Aaps Journal, 2005. 7(4): p. E885-E894.

108. Sugano, N., et al., Nicotine inhibits the production of inflammatory mediators in U937 cells through modulation of nuclear factor-kappaB activation. Biochem Biophys Res Commun, 1998. 252(1): p. 25-8.

109. Wang, H., et al., Nicotinic acetylcholine receptor alpha7 subunit is an essential regulator of inflammation. Nature, 2003. 421(6921): p. 384-8.

110. Yoshikawa, H., et al., Nicotine inhibits the production of proinflammatory mediators in human monocytes by suppression of I-kappa $B$ phosphorylation and nuclear factor-kappa B transcriptional activity through nicotinic acetylcholine receptor alpha 7. Clinical and Experimental Immunology, 2006. 146(1): p. 116-123.

111. Tracey, K.J., The inflammatory reflex. Nature, 2002. 420(6917): p. 853-9.

112. Pinheiro, N.M., et al., Acute lung injury is reduced by the alpha7nAChR agonist PNU-282987 through changes in the macrophage profile. FASEB J, 2016. 
113. $\mathrm{Xu}, \mathrm{Y}$., et al., Cigarette smoke (CS) and nicotine delay neutrophil spontaneous death via suppressing production of diphosphoinositol pentakisphosphate. Proc Natl Acad Sci U S A, 2013. 110(19): p. 7726-31.

114. Seow, W.K., et al., Nicotine-induced release of elastase and eicosanoids by human neutrophils. Inflammation, 1994. 18(2): p. 119-27.

115. Hosseinzadeh, A., et al., Nicotine induces neutrophil extracellular traps. J Leukoc Biol, 2016. 100(5): p. 1105-1112.

116. Fernandez, I.E. and O. Eickelberg, The impact of TGF-beta on lung fibrosis: from targeting to biomarkers. Proc Am Thorac Soc, 2012. 9(3): p. $111-6$.

117. Ask, K., et al., Progressive pulmonary fibrosis is mediated by TGF-beta isoform 1 but not TGF-beta3. Int J Biochem Cell Biol, 2008. 40(3): p. 48495.

118. Liu, X., Inflammatory cytokines augments TGF-beta1-induced epithelialmesenchymal transition in A549 cells by up-regulating TbetaR-I. Cell Motil Cytoskeleton, 2008. 65(12): p. 935-44.

119. Kendall, R.T. and C.A. Feghali-Bostwick, Fibroblasts in fibrosis: novel roles and mediators. Front Pharmacol, 2014. 5: p. 123.

120. Kolb, M., et al., Transient expression of IL-1beta induces acute lung injury and chronic repair leading to pulmonary fibrosis. J Clin Invest, 2001. 107(12): p. 1529-36. 
121. Cavarra, E., et al., Early response to bleomycin is characterized by different cytokine and cytokine receptor profiles in lungs. Am J Physiol Lung Cell Mol Physiol, 2004. 287(6): p. L1186-92.

122. Wynn, T.A., Integrating mechanisms of pulmonary fibrosis. J Exp Med, 2011. 208(7): p. 1339-50.

123. Wynn, T.A., Fibrotic disease and the $T(H) 1 / T(H) 2$ paradigm. Nat Rev Immunol, 2004. 4(8): p. 583-94.

124. Romagnani, S., T-cell subsets (Th1 versus Th2). Ann Allergy Asthma Immunol, 2000. 85(1): p. 9-18; quiz 18, 21.

125. Boveda-Ruiz, D., et al., Differential role of regulatory $T$ cells in early and late stages of pulmonary fibrosis. Immunobiology, 2013. 218(2): p. 245254.

126. Huen, S.C., G.W. Moeckel, and L.G. Cantley, Macrophage-specific deletion of transforming growth factor-beta1 does not prevent renal fibrosis after severe ischemia-reperfusion or obstructive injury. Am J Physiol Renal Physiol, 2013. 305(4): p. F477-84.

127. Kruger, P., et al., Neutrophils: Between host defence, immune modulation, and tissue injury. PLoS Pathog, 2015. 11(3): p. e1004651.

128. Manoury, B., et al., Influence of early neutrophil depletion on MMPS/TIMP1 balance in bleomycin-induced lung fibrosis. Int Immunopharmacol, 2007. 7(7): p. 900-11. 
129. Wright, D.B., H. Meurs, and B.G. Dekkers, Integrins: therapeutic targets in airway hyperresponsiveness and remodelling? Trends Pharmacol Sci, 2014. 35(11): p. 567-74.

130. Manon-Jensen, T., N.G. Kjeld, and M.A. Karsdal, Collagen-mediated hemostasis. J Thromb Haemost, 2016. 14(3): p. 438-48.

131. Eckes, B., et al., Mechanical tension and integrin alpha 2 beta 1 regulate fibroblast functions. J Investig Dermatol Symp Proc, 2006. 11(1): p. 66-72.

132. Zeltz, C. and D. Gullberg, The integrin-collagen connection - a glue for tissue repair? J Cell Sci, 2016. 129(6): p. 1284.

133. Stahl, M., et al., Lung collagens perpetuate pulmonary fibrosis via CD204 and M2 macrophage activation. PLoS One, 2013. 8(11): p. e81382.

134. Prasse, A., et al., A vicious circle of alveolar macrophages and fibroblasts perpetuates pulmonary fibrosis via CCL18. Am J Respir Crit Care Med, 2006. 173(7): p. 781-92.

135. Murthy, S., et al., Alternative activation of macrophages and pulmonary fibrosis are modulated by scavenger receptor, macrophage receptor with collagenous structure. FASEB J, 2015. 29(8): p. 3527-36.

136. Xia, H., et al., Low alpha(2)beta(1) integrin function enhances the proliferation of fibroblasts from patients with idiopathic pulmonary fibrosis by activation of the beta-catenin pathway. Am J Pathol, 2012. 181(1): p. 222-33.

137. Halliwell, B. and J.M.C. Gutteridge, Free radicals in biology and medicine. 1989: Clarendon Press. 
138. Blokhina, O., E. Virolainen, and K.V. Fagerstedt, Antioxidants, oxidative damage and oxygen deprivation stress: a review. Ann Bot, 2003. 91 Spec No: p. $179-94$.

139. Bowler, R.P. and J.D. Crapo, Oxidative stress in airways: is there a role for extracellular superoxide dismutase? Am J Respir Crit Care Med, 2002. 166(12 Pt 2): p. S38-43.

140. McCord, J.M. and I. Fridovich, Superoxide dismutase. An enzymic function for erythrocuprein (hemocuprein). J Biol Chem, 1969. 244(22): p. 6049-55.

141. Weisiger, R.A. and I. Fridovich, Mitochondrial superoxide simutase. Site of synthesis and intramitochondrial localization. J Biol Chem, 1973. 248(13): p. 4793-6.

142. Marklund, S.L., Human copper-containing superoxide dismutase of high molecular weight. Proc Natl Acad Sci U S A, 1982. 79(24): p. 7634-8.

143. Crapo, J.D., et al., Copper,zinc superoxide dismutase is primarily a cytosolic protein in human cells. Proc Natl Acad Sci U S A, 1992. 89(21): p. $10405-9$.

144. Weisiger, R.A. and I. Fridovich, Superoxide dismutase. Organelle specificity. J Biol Chem, 1973. 248(10): p. 3582-92.

145. Robinson, B.H., The role of manganese superoxide dismutase in health and disease. J Inherit Metab Dis, 1998. 21(5): p. 598-603. 
146. Kinnula, V.L., et al., Oxidative stress in pulmonary fibrosis: a possible role for redox modulatory therapy. Am J Respir Crit Care Med, 2005. 172(4): p. $417-22$.

147. Montuschi, P., et al., 8-Isoprostane as a biomarker of oxidative stress in interstitial lung diseases. Am J Respir Crit Care Med, 1998. 158(5 Pt 1): p. $1524-7$.

148. Cantin, A.M., et al., Oxidant-mediated epithelial cell injury in idiopathic pulmonary fibrosis. J Clin Invest, 1987. 79(6): p. 1665-73.

149. Hallgren, R., et al., The eosinophil component of the alveolitis in idiopathic pulmonary fibrosis. Signs of eosinophil activation in the lung are related to impaired lung function. Am Rev Respir Dis, 1989. 139(2): p. 373-7.

150. Lakari, E., et al., Inducible nitric oxide synthase, but not xanthine oxidase, is highly expressed in interstitial pneumonias and granulomatous diseases of human lung. Am J Clin Pathol, 2002. 117(1): p. 132-42.

151. Dhouib, H., et al., Oxidative damage and histopathological changes in lung of rat chronically exposed to nicotine alone or associated to ethanol. Pathol Biol (Paris), 2015. 63(6): p. 258-67.

152. Toledano, A., M.I. Alvarez, and A. Toledano-Diaz, Variability in the effects of nicotine on different regions of the brain: changes in the concentration of superoxide dismutase isoforms. Cent Nerv Syst Agents Med Chem, 2014. 14(1): p. 10-22. 
153. Bowler, R.P., et al., Role of extracellular superoxide dismutase in bleomycin-induced pulmonary fibrosis. Am J Physiol Lung Cell Mol Physiol, 2002. 282(4): p. L719-26.

154. Heikkila-Laurila, H.P. and M.M. Rajamaki, Idiopathic pulmonary fibrosis in West Highland white terriers. Vet Clin North Am Small Anim Pract, 2014. 44(1): p. 129-42.

155. Williams, K., et al., Identification of spontaneous feline idiopathic pulmonary fibrosis: morphology and ultrastructural evidence for a type II pneumocyte defect. Chest, 2004. 125(6): p. 2278-88.

156. Cohn, L.A., et al., Identification and characterization of an idiopathic pulmonary fibrosis-like condition in cats. J Vet Intern Med, 2004. 18(5): p. $632-41$.

157. Miele, A., et al., Chronic pleuropulmonary fibrosis and elastosis of aged donkeys: similarities to human pleuroparenchymal fibroelastosis. Chest, 2014. 145(6): p. 1325-32.

158. Williams, K.J., et al., Equine multinodular pulmonary fibrosis: a newly recognized herpesvirus-associated fibrotic lung disease. Vet Pathol, 2007. 44(6): p. 849-62.

159. Williams, K. and J. Roman, Studying human respiratory disease in animals--role of induced and naturally occurring models. J Pathol, 2016. 238(2): p. 220-32.

160. Izbicki, G., et al., Time course of bleomycin-induced lung fibrosis. Int J Exp Pathol, 2002. 83(3): p. 111-9. 
161. Sebti, S.M., et al., Bleomycin hydrolase: molecular cloning, sequencing, and biochemical studies reveal membership in the cysteine proteinase family. Biochemistry, 1989. 28(16): p. 6544-8.

162. Umezawa, H., et al., Studies on bleomycin. Cancer, 1967. 20(5): p. 891-5.

163. Yagoda, A., et al., Bleomycin, an antitumor antibiotic. Clinical experience in 274 patients. Ann Intern Med, 1972. 77(6): p. 861-70.

164. Swiderski, R.E., et al., Differential expression of extracellular matrix remodeling genes in a murine model of bleomycin-induced pulmonary fibrosis. Am J Pathol, 1998. 152(3): p. 821-8.

165. Liu, F. and D.J. Tschumperlin, Micro-mechanical characterization of lung tissue using atomic force microscopy. J Vis Exp, 2011(54).

166. Ebihara, T., et al., Changes in extracellular matrix and tissue viscoelasticity in bleomycin-induced lung fibrosis. Temporal aspects. Am J Respir Crit Care Med, 2000. 162(4 Pt 1): p. 1569-76.

167. Liu, T., et al., Regulation of found in inflammatory zone 1 expression in bleomycin-induced lung fibrosis: role of IL-4/IL-13 and mediation via STAT-6. J Immunol, 2004. 173(5): p. 3425-31.

168. Pechkovsky, D.V., et al., Alternatively activated alveolar macrophages in pulmonary fibrosis-mediator production and intracellular signal transduction. Clin Immunol, 2010. 137(1): p. 89-101.

169. Kim, K.K., et al., Alveolar epithelial cell mesenchymal transition develops in vivo during pulmonary fibrosis and is regulated by the extracellular matrix. Proc Natl Acad Sci U S A, 2006. 103(35): p. 13180-5. 
170. Liu, G., et al., miR-21 mediates fibrogenic activation of pulmonary fibroblasts and lung fibrosis. J Exp Med, 2010. 207(8): p. 1589-97.

171. Mouratis, M.A. and V. Aidinis, Modeling pulmonary fibrosis with bleomycin. Curr Opin Pulm Med, 2011. 17(5): p. 355-61.

172. Moore, B.B. and C.M. Hogaboam, Murine models of pulmonary fibrosis. Am J Physiol Lung Cell Mol Physiol, 2008. 294(2): p. L152-60.

173. Osanai, K., et al., The effect of cigarette smoke on bleomycin-induced pulmonary fibrosis in hamsters. Am Rev Respir Dis, 1988. 138(5): p. 1276-81.

174. Cisneros-Lira, J., et al., Cigarette smoke exposure potentiates bleomycininduced lung fibrosis in guinea pigs. American journal of physiology. Lung cellular and molecular physiology, 2003. 285(4): p. L949-56.

175. Cisneros-Lira, J., et al., Cigarette smoke exposure potentiates bleomycininduced lung fibrosis in guinea pigs. Am J Physiol Lung Cell Mol Physiol, 2003. 285(4): p. L949-56.

176. Teke, T., et al., Cigarette smoke and bleomycin-induced pulmonary oxidative stress in rats. Exp Ther Med, 2012. 4(1): p. 121-124.

177. Tee, S.Y., et al., Cell Shape and Substrate Rigidity Both Regulate Cell Stiffness. Biophysical Journal, 2011. 100(3): p. 303-303.

178. Smith, P.G., et al., Mechanical strain increases cell stiffness through cytoskeletal filament reorganization. American Journal of Physiology-Lung Cellular and Molecular Physiology, 2003. 285(2): p. L456-L463. 
179. Lo, C.M., et al., Cell movement is guided by the rigidity of the substrate. Biophysical Journal, 2000. 79(1): p. 144-152.

180. Pelham, R.J. and Y.L. Wang, Cell locomotion and focal adhesions are regulated by substrate flexibility (vol 94, pg 13661, 1997). Proceedings of the National Academy of Sciences of the United States of America, 1998. 95(20): p. 12070-12070.

181. Wong, J.Y., et al., Directed movement of vascular smooth muscle cells on gradient-compliant hydrogels. Langmuir, 2003. 19(5): p. 1908-1913.

182. Discher, D.E., P. Janmey, and Y.L. Wang, Tissue cells feel and respond to the stiffness of their substrate. Science, 2005. 310(5751): p. 1139-43.

183. Engler, A.J., et al., Matrix elasticity directs stem cell lineage specification. Cell, 2006. 126(4): p. 677-689.

184. Mckee, C.T., et al., The effect of biophysical attributes of the ocular trabecular meshwork associated with glaucoma on the cell response to therapeutic agents. Biomaterials, 2011. 32(9): p. 2417-2423.

185. Shifren, A. and R.P. Mecham, The stumbling block in lung repair of emphysema: elastic fiber assembly. Proc Am Thorac Soc, 2006. 3(5): p. 428-33.

186. Senior, R.M., D.R. Bielefeld, and M.K. Abensohn, The effects of proteolytic enzymes on the tensile strength of human lung. Am Rev Respir Dis, 1975. 111(2): p. 184-8.

187. White, E.S., Lung extracellular matrix and fibroblast function. Ann Am Thorac Soc, 2015. 12 Suppl 1: p. S30-3. 
188. Laurent, G.J., Rates of collagen synthesis in lung, skin and muscle obtained in vivo by a simplified method using [3H]proline. Biochem J, 1982. 206(3): p. 535-44.

189. Shapiro, S.D., et al., Marked longevity of human lung parenchymal elastic fibers deduced from prevalence of $D$-aspartate and nuclear weaponsrelated radiocarbon. J Clin Invest, 1991. 87(5): p. 1828-34.

190. Chapman, H.A., Disorders of lung matrix remodeling. J Clin Invest, 2004. 113(2): p. 148-57.

191. Yeung, T., et al., Effects of substrate stiffness on cell morphology, cytoskeletal structure, and adhesion. Cell Motil Cytoskeleton, 2005. 60(1): p. 24-34.

192. Wipff, P.J., et al., Myofibroblast contraction activates latent TGF-beta1 from the extracellular matrix. J Cell Biol, 2007. 179(6): p. 1311-23.

193. Lo, C.M., et al., Cell movement is guided by the rigidity of the substrate. Biophys J, 2000. 79(1): p. 144-52.

194. Peyton, S.R. and A.J. Putnam, Extracellular matrix rigidity governs smooth muscle cell motility in a biphasic fashion. J Cell Physiol, 2005. 204(1): p. 198-209.

195. Karsdal, M.A., et al., Extracellular Matrix Remodeling: The Common Denominator in Connective Tissue Diseases Possibilities for Evaluation and Current Understanding of the Matrix as More Than a Passive Architecture, but a Key Player in Tissue Failure. Assay and Drug Development Technologies, 2013. 11(2): p. 70-92. 
196. Liu, F., et al., Feedback amplification of fibrosis through matrix stiffening and COX-2 suppression. J Cell Biol, 2010. 190(4): p. 693-706.

197. Cukierman, E., Cell migration analyses within fibroblast-derived 3-D matrices. Methods Mol Biol, 2005. 294: p. 79-93.

198. Burns, A.R., C.W. Smith, and D.C. Walker, Unique structural features that influence neutrophil emigration into the lung. Physiol Rev, 2003. 83(2): $p$. 309-36.

199. Poobalarahi, F., C.F. Baicu, and A.D. Bradshaw, Cardiac myofibroblasts differentiated in 3D culture exhibit distinct changes in collagen I production, processing, and matrix deposition. American Journal of Physiology-Heart and Circulatory Physiology, 2006. 291(6): p. H2924H2932.

200. Mao, Y. and J.E. Schwarzbauer, Stimulatory effects of a threedimensional microenvironment on cell-mediated fibronectin fibrillogenesis. Journal of Cell Science, 2005. 118(19): p. 4427-4436.

201. Huh, D., et al., Reconstituting Organ-Level Lung Functions on a Chip. Science, 2010. 328(5986): p. 1662-1668.

202. Petersen, T.H., et al., Matrix composition and mechanics of decellularized lung scaffolds. Cells Tissues Organs, 2012. 195(3): p. 222-31.

203. Nichols, J.E., et al., Production and assessment of decellularized pig and human lung scaffolds. Tissue Eng Part A, 2013. 19(17-18): p. 2045-62. 
204. Melo, E., et al., Effects of the decellularization method on the local stiffness of acellular lungs. Tissue Eng Part C Methods, 2014. 20(5): p. $412-22$.

205. Binnig, G., C.F. Quate, and C. Gerber, Atomic force microscope. Phys Rev Lett, 1986. 56(9): p. 930-933.

206. Chang, Y.R., et al., Automated AFM force curve analysis for determining elastic modulus of biomaterials and biological samples. J Mech Behav Biomed Mater, 2014. 37: p. 209-18.

207. Cavalcante, F.S., et al., Mechanical interactions between collagen and proteoglycans: implications for the stability of lung tissue. J Appl Physiol (1985), 2005. 98(2): p. 672-9.

208. Butcher, D.T., T. Alliston, and V.M. Weaver, A tense situation: forcing tumour progression. Nat Rev Cancer, 2009. 9(2): p. 108-22.

209. Parameswaran, H., A. Majumdar, and B. Suki, Linking Microscopic Spatial Patterns of Tissue Destruction in Emphysema to Macroscopic Decline in Stiffness Using a 3D Computational Model. Plos Computational Biology, 2011. 7(4).

210. Liu, F., et al., Feedback amplification of fibrosis through matrix stiffening and COX-2 suppression. Journal of Cell Biology, 2010. 190(4): p. 693706.

211. Roman, J., et al., Ethanol stimulates the expression of fibronectin in lung fibroblasts via kinase-dependent signals that activate CREB. Am J Physiol Lung Cell Mol Physiol, 2005. 288(5): p. L975-87. 
212. Burnham, E.L., et al., Increased fibronectin expression in lung in the setting of chronic alcohol abuse. Alcohol Clin Exp Res, 2007. 31(4): p. $675-83$.

213. Brown, L.A., et al., Alveolar type I/ cells from ethanol-fed rats produce a fibronectin-enriched extracellular matrix that promotes monocyte activation. Alcohol, 2007. 41(5): p. 317-24.

214. Ritzenthaler, J. and J. Roman, Differential effects of protein kinase C inhibitors on fibronectin-induced interleukin-beta gene transcription, protein synthesis and secretion in human monocytic cells. Immunology, 1998. 95(2): p. 264-71.

215. Crapo, J.D., J.M. McCord, and I. Fridovich, Preparation and assay of superoxide dismutases. Methods Enzymol, 1978. 53: p. 382-93.

216. Rowell, P.P. and M.J. Clark, The effect of chronic oral nicotine administration on fetal weight and placental amino acid accumulation in mice. Toxicol Appl Pharmacol, 1982. 66(1): p. 30-8.

217. Rowell, P.P., et al., Oral administration of nicotine: its uptake and distribution after chronic administration to mice. J Pharmacol Methods, 1983. 9(4): p. 249-61.

218. Christensen, P.J., et al., Pneumocystis murina infection and cigarette smoke exposure interact to cause increased organism burden, development of airspace enlargement, and pulmonary inflammation in mice. Infect Immun, 2008. 76(8): p. 3481-90. 
219. Hautamaki, R.D., et al., Requirement for macrophage elastase for cigarette smoke-induced emphysema in mice. Science, 1997. 277(5334): p. 2002-4.

220. Shapiro, S.D., Animal models for chronic obstructive pulmonary disease: age of klotho and marlboro mice. Am J Respir Cell Mol Biol, 2000. 22(1): p. 4-7.

221. Ashcroft, T., J.M. Simpson, and V. Timbrell, Simple method of estimating severity of pulmonary fibrosis on a numerical scale. J Clin Pathol, 1988. 41(4): p. 467-70.

222. Kolachala, V.L., et al., Epithelial-derived fibronectin expression, signaling, and function in intestinal inflammation. J Biol Chem, 2007. 282(45): p. 32965-73.

223. Rodarte, J.R., Stress-Strain Analysis and the Lung. Federation Proceedings, 1982. 41(1): p. 130-135.

224. Idiopathic Pulmonary Fibrosis Clinical Research, N., et al., Prednisone, azathioprine, and $\mathrm{N}$-acetylcysteine for pulmonary fibrosis. $\mathrm{N}$ Engl J Med, 2012. 366(21): p. 1968-77.

225. Team, R.C., R: A language and environment for statistical computing. 2013.

226. Organization, W.H., WHO report on the global tobacco epidemic, 2011: warning about the dangers of tobacco. MPOWER, 2011. 
227. Totti, N., et al., Nicotine is chemotactic for neutrophils and enhances neutrophil responsiveness to chemotactic peptides. Science, 1984. 223(4632): p. 169-171.

228. Schuller, H.M., Is cancer triggered by altered signalling of nicotinic acetylcholine receptors? Nat Rev Cancer, 2009. 9(3): p. 195-205.

229. Vassallo, R., et al., Nicotine and oxidative cigarette smoke constituents induce immune-modulatory and pro-inflammatory dendritic cell responses. Mol Immunol, 2008. 45(12): p. 3321-9.

230. D'Armiento, J., et al., Collagenase expression in the lungs of transgenic mice causes pulmonary emphysema. Cell, 1992. 71(6): p. 955-61.

231. Wong, S., M.G. Belvisi, and M.A. Birrell, MMP/TIMP expression profiles in distinct lung disease models: implications for possible future therapies. Respir Res, 2009. 10: p. 72.

232. Moore, B.B., et al., CCR2-mediated recruitment of fibrocytes to the alveolar space after fibrotic injury. Am J Pathol, 2005. 166(3): p. 675-84.

233. Ooi, C.Y., et al., The role of collagen in extralobar pulmonary artery stiffening in response to hypoxia-induced pulmonary hypertension. Am J Physiol Heart Circ Physiol, 2010. 299(6): p. H1823-31.

234. Shintani, Y., et al., Collagen I promotes epithelial-to-mesenchymal transition in lung cancer cells via transforming growth factor-beta signaling. Am J Respir Cell Mol Biol, 2008. 38(1): p. 95-104.

235. Laskin, D.L., et al., Chemotactic activity of collagen-like polypeptides for human peripheral blood neutrophils. J Leukoc Biol, 1986. 39(3): p. 255-66. 
236. Schuller, H.M., et al., Interaction of tobacco-specific toxicants with the neuronal alpha(7) nicotinic acetylcholine receptor and its associated mitogenic signal transduction pathway: potential role in lung carcinogenesis and pediatric lung disorders. Eur J Pharmacol, 2000. 393(1-3): p. 265-77.

237. Spindel, E.R., Is nicotine the estrogen of lung cancer? Am J Respir Crit Care Med, 2009. 179(12): p. 1081-2.

238. Wongtrakool, C., et al., Prenatal nicotine exposure alters lung function and airway geometry through alpha7 nicotinic receptors. Am J Respir Cell Mol Biol, 2012. 46(5): p. 695-702.

239. Toyohara, J. and K. Hashimoto, alpha7 Nicotinic Receptor Agonists: Potential Therapeutic Drugs for Treatment of Cognitive Impairments in Schizophrenia and Alzheimer's Disease. Open Med Chem J, 2010. 4: p. 37-56.

240. Ghosh, A.K., et al., Smad-dependent stimulation of type I collagen gene expression in human skin fibroblasts by TGF-beta involves functional cooperation with p300/CBP transcriptional coactivators. Oncogene, 2000. 19(31): p. 3546-55.

241. Dobaczewski, M., et al., Smad3 signaling critically regulates fibroblast phenotype and function in healing myocardial infarction. Circ Res, 2010. 107(3): p. 418-28.

242. Ritzenthaler, J.D. and J. Roman, Interleukin-1 beta gene transcription in U937 cells is modulated by type I collagen and cytoskeletal integrity via 
distinct signaling pathways. Journal of Interferon and Cytokine Research, 2001. 21(2): p. 105-116.

243. Gullberg, D., et al., Analysis of alpha 1 beta 1, alpha 2 beta 1 and alpha 3 beta 1 integrins in cell--collagen interactions: identification of conformation dependent alpha 1 beta 1 binding sites in collagen type I. EMBO J, 1992. 11(11): p. 3865-73.

244. Dustin, M.L. and A.R. de Fougerolles, Reprogramming T cells: the role of extracellular matrix in coordination of $T$ cell activation and migration. Curr Opin Immunol, 2001. 13(3): p. 286-90.

245. Krieglstein, C.F., et al., Collagen-binding integrin alpha1beta1 regulates intestinal inflammation in experimental colitis. J Clin Invest, 2002. 110(12): p. $1773-82$.

246. Lois, M., et al., Ethanol Ingestion Increases Activation of Matrix Metalloproteinases in Rat Lungs during Acute Endotoxemia. American Journal of Respiratory and Critical Care Medicine, 1999. 160(4): p. 13541360.

247. Ramirez, A.M., et al., Activation of Tissue Remodeling Precedes Obliterative Bronchiolitis in Lung Transplant Recipients. Biomark Insights, 2008. 3: p. 351-359.

248. Muro, A.F., et al., An essential role for fibronectin extra type III domain A in pulmonary fibrosis. Am J Respir Crit Care Med, 2008. 177(6): p. 638-45. 
249. Barkan, D., J.E. Green, and A.F. Chambers, Extracellular matrix: a gatekeeper in the transition from dormancy to metastatic growth. Eur $\mathrm{J}$ Cancer, 2010. 46(7): p. 1181-8.

250. Park, S.J., et al., Abstract B16: The effect of e-cigarette exposure on airway epithelial cell gene expression and transformation. Clinical Cancer Research, 2014. 20(2 Supplement): p. B16.

251. Dutra, L.M. and S.A. Glantz, Electronic cigarettes and conventional cigarette use among us adolescents: A cross-sectional study. JAMA Pediatrics, 2014.

252. Fairchild, A.L., R. Bayer, and J. Colgrove, The renormalization of smoking? E-cigarettes and the tobacco "endgame". N Engl J Med, 2014. 370(4): p. 293-5.

253. Zhang, H.Y., et al., Lung fibroblast alpha-smooth muscle actin expression and contractile phenotype in bleomycin-induced pulmonary fibrosis. Am J Pathol, 1996. 148(2): p. 527-37.

254. Maouche, K., et al., \{alpha\}7 nicotinic acetylcholine receptor regulates airway epithelium differentiation by controlling basal cell proliferation. Am J Pathol, 2009. 175(5): p. 1868-82.

255. Annoni, R., et al., Extracellular matrix composition in COPD. Eur Respir J, 2012. 40(6): p. 1362-73.

256. Sand, J.M., et al., High levels of biomarkers of collagen remodeling are associated with increased mortality in COPD - results from the ECLIPSE study. Respir Res, 2016. 17(1): p. 125. 
257. Jamal, A., et al., Current cigarette smoking among adults - United States, 2005-2014. MMWR Morb Mortal Wkly Rep, 2015. 64(44): p. 1233-40.

258. Sciences, U.D.o.H.a.H., The Health Consequences of Smoking-50 Years of Progress: A Report of the Surgeon General, in The Health Consequences of Smoking-50 Years of Progress: A Report of the Surgeon General. 2014, US Department of Health and Human Sciences, Centers for Disease Control and Prevention: Atlanta (GA).

259. Schroeder, M.J. and A.C. Hoffman, Electronic cigarettes and nicotine clinical pharmacology. Tob Control, 2014. 23 Suppl 2: p. ii30-5.

260. King, B.A., et al., Trends in awareness and use of electronic cigarettes among US adults, 2010-2013. Nicotine Tob Res, 2015. 17(2): p. 219-27.

261. Albuquerque, E.X., et al., Mammalian nicotinic acetylcholine receptors: from structure to function. Physiol Rev, 2009. 89(1): p. 73-120.

262. Martin, W.J., 2nd and D.L. Kachel, Bleomycin-induced pulmonary endothelial cell injury: evidence for the role of iron-catalyzed toxic oxygenderived species. J Lab Clin Med, 1987. 110(2): p. 153-8.

263. Vyalov, S.L., G. Gabbiani, and Y. Kapanci, Rat alveolar myofibroblasts acquire alpha-smooth muscle actin expression during bleomycin-induced pulmonary fibrosis. Am J Pathol, 1993. 143(6): p. 1754-65.

264. Giri, S.N., et al., Increases in lung prolyl hydroxylase and superoxide dismutase activities during bleomycin-induced lung fibrosis in hamsters. Exp Mol Pathol, 1983. 39(3): p. 317-26. 
265. Yamazaki, C., et al., Production of superoxide and nitric oxide by alveolar macrophages in the bleomycin-induced interstitial pneumonia mice model. Jpn J Pharmacol, 1998. 78(1): p. 69-73.

266. Mungunsukh, O., et al., Bleomycin induces the extrinsic apoptotic pathway in pulmonary endothelial cells. Am J Physiol Lung Cell Mol Physiol, 2010. 298(5): p. L696-703.

267. Chaudhary, N.I., A. Schnapp, and J.E. Park, Pharmacologic differentiation of inflammation and fibrosis in the rat bleomycin model. Am J Respir Crit Care Med, 2006. 173(7): p. 769-76.

268. Chandrakasan, G. and R.S. Bhatnagar, Stimulation of collagen synthesis in fibroblast cultures by superoxide. Cell Mol Biol, 1991. 37(7): p. 751-5.

269. He, C., et al., Mitochondrial Cu,Zn-superoxide dismutase mediates pulmonary fibrosis by augmenting H2O2 generation. J Biol Chem, 2011. 286(17): p. 15597-607.

270. B, B.M., et al., Animal models of fibrotic lung disease. Am J Respir Cell Mol Biol, 2013. 49(2): p. 167-79.

271. Peng, R., et al., Bleomycin induces molecular changes directly relevant to idiopathic pulmonary fibrosis: a model for "active" disease. PLoS One, 2013. 8(4): p. e59348.

272. Mata, M., et al., Oral N-acetylcysteine reduces bleomycin-induced lung damage and mucin Muc5ac expression in rats. Eur Respir J, 2003. 22(6): p. $900-5$. 
273. Cortijo, J., et al., Attenuation by oral N-acetylcysteine of bleomycininduced lung injury in rats. Eur Respir J, 2001. 17(6): p. 1228-35.

274. Serrano-Mollar, A., et al., P-selectin upregulation in bleomycin induced lung injury in rats: effect of N-acetyl-L-cysteine. Thorax, 2002. 57(7): p. 629-34.

275. Iraz, M., et al., Ginkgo biloba inhibits bleomycin-induced lung fibrosis in rats. Pharmacol Res, 2006. 53(3): p. 310-6.

276. Teixeira, K.C., et al., Attenuation of bleomycin-induced lung injury and oxidative stress by $\mathrm{N}$-acetylcysteine plus deferoxamine. Pulm Pharmacol Ther, 2008. 21(2): p. 309-16.

277. Santos-Silva, M.A., et al., Redox imbalance and pulmonary function in bleomycin-induced fibrosis in C57BL/6, DBA/2, and BALB/c mice. Toxicol Pathol, 2012. 40(5): p. 731-41.

278. Pabst, M.J., et al., Inhibition of neutrophil and monocyte defensive functions by nicotine. J Periodontol, 1995. 66(12): p. 1047-55.

279. Yoshikawa, H., et al., Nicotine inhibits the production of proinflammatory mediators in human monocytes by suppression of I-kappaB phosphorylation and nuclear factor-kappaB transcriptional activity through nicotinic acetylcholine receptor alpha7. Clin Exp Immunol, 2006. 146(1): p. 116-23.

280. de Jonge, W.J. and L. Ulloa, The alpha7 nicotinic acetylcholine receptor as a pharmacological target for inflammation. Br J Pharmacol, 2007. 151(7): p. 915-29. 
281. Tanaka, K., et al., Effects of lecithinized superoxide dismutase and/or pirfenidone against bleomycin-induced pulmonary fibrosis. Chest, 2012. 142(4): p. 1011-9.

282. Galvan, L., et al., Inhibition of bleomycin-induced DNA breakage by superoxide dismutase. Cancer Res, 1981. 41(12 Pt 1): p. 5103-6.

283. Chen, J.F., et al., Improved mitochondrial function underlies the protective effect of pirfenidone against tubulointerstitial fibrosis in 5/6 nephrectomized rats. PLoS One, 2013. 8(12): p. e83593.

284. Flouris, A.D., et al., Acute impact of active and passive electronic cigarette smoking on serum cotinine and lung function. Inhal Toxicol, 2013. 25(2): p. $91-101$.

285. Raghu, G., et al., Incidence and prevalence of idiopathic pulmonary fibrosis. Am J Respir Crit Care Med, 2006. 174(7): p. 810-6.

286. Belkin, A. and J.J. Swigris, Patient expectations and experiences in idiopathic pulmonary fibrosis: implications of patient surveys for improved care. Expert Rev Respir Med, 2014. 8(2): p. 173-8.

287. Bonella, F., S. Stowasser, and L. Wollin, Idiopathic pulmonary fibrosis: current treatment options and critical appraisal of nintedanib. Drug Des Devel Ther, 2015. 9: p. 6407-19.

288. Noble, P.W., et al., Pirfenidone for idiopathic pulmonary fibrosis: analysis of pooled data from three multinational phase 3 trials. Eur Respir J, 2016. 47(1): p. 243-53. 
289. Oldham, J.M. and I. Noth, Idiopathic pulmonary fibrosis: early detection and referral. Respir Med, 2014. 108(6): p. 819-29.

290. Nathan, S.D., et al., Validation of test performance characteristics and minimal clinically important difference of the 6-minute walk test in patients with idiopathic pulmonary fibrosis. Respir Med, 2015. 109(7): p. 914-22.

291. Ley, B., H.R. Collard, and T.E. King, Jr., Clinical course and prediction of survival in idiopathic pulmonary fibrosis. Am J Respir Crit Care Med, 2011. 183(4): p. 431-40.

292. Russell, A.M., et al., Daily Home Spirometry: An Effective Tool for Detecting Progression in Idiopathic Pulmonary Fibrosis. Am J Respir Crit Care Med, 2016. 194(8): p. 989-997.

293. Li, X., et al., Relevance analysis of clinical and lung function parameters changing and prognosis of idiopathic pulmonary fibrosis. Int J Clin Exp Med, 2014. 7(12): p. 4759-69.

294. Nishiyama, O., et al., Prognostic value of forced expiratory volume in 1 second/forced vital capacity in idiopathic pulmonary fibrosis. Chron Respir Dis, 2016. 13(1): p. 40-7.

295. Cai, M., et al., Clinical features and outcomes of 210 patients with idiopathic pulmonary fibrosis. Chin Med J (Engl), 2014. 127(10): p. 186873.

296. Brown, A.W., et al., Dynamic patient counseling: a novel concept in idiopathic pulmonary fibrosis. Chest, 2012. 142(4): p. 1005-10. 
297. Schwartz, D.A., et al., Determinants of survival in idiopathic pulmonary fibrosis. Am J Respir Crit Care Med, 1994. 149(2 Pt 1): p. 450-4.

298. Wells, A.U., et al., Idiopathic pulmonary fibrosis: a composite physiologic index derived from disease extent observed by computed tomography. Am J Respir Crit Care Med, 2003. 167(7): p. 962-9.

299. Nishiyama, O. and Y. Tohda, Obstructive lung function in idiopathic pulmonary fibrosis. Chron Respir Dis, 2016. 13(2): p. 206.

300. Fulmer, J.D., et al., Small airways in idiopathic pulmonary fibrosis. Comparison of morphologic and physiologic observations. J Clin Invest, 1977. 60(3): p. 595-610.

301. Ostrow, D. and R.M. Cherniack, Resistance to airflow in patients with diffuse interstitial lung disease. Am Rev Respir Dis, 1973. 108(2): p. 20510.

302. Yernault, J.C., et al., Pulmonary mechanics in diffuse fibrosing alveolitis. Bull Physiopathol Respir (Nancy), 1975. 11(2): p. 231-44.

303. Cherniack, R.M., et al., Correlation of structure and function in idiopathic pulmonary fibrosis. Am J Respir Crit Care Med, 1995. 151(4): p. 1180-8.

304. Mura, M., et al., The Presence of Emphysema Further Impairs Physiologic Function in Patients With Idiopathic Pulmonary Fibrosis. Respiratory Care, 2006. 51(3): p. 257-265.

305. Ashley, S.L., et al., Six-SOMAmer Index Relating to Immune, Protease and Angiogenic Functions Predicts Progression in IPF. PLoS One, 2016. 11(8): p. e0159878. 
306. Yousaf, S., et al., Identification and clinical characterization of HermanskyPudlak syndrome alleles in the Pakistani population. Pigment Cell Melanoma Res, 2016. 29(2): p. 231-5.

307. Gahl, W.A. and M. Huizing, Hermansky-Pudlak Syndrome, in GeneReviews(R), R.A. Pagon, et al., Editors. 1993: Seattle (WA).

308. Santiago Borrero, P.J., et al., Genetic testing for oculocutaneous albinism type 1 and 2 and Hermansky-Pudlak syndrome type 1 and 3 mutations in Puerto Rico. J Invest Dermatol, 2006. 126(1): p. 85-90.

309. Witkop, C.J., et al., Hermansky-Pudlak syndrome (HPS). An epidemiologic study. Ophthalmic Paediatr Genet, 1990. 11(3): p. 245-50.

310. Hermansky, F. and P. Pudlak, Albinism associated with hemorrhagic diathesis and unusual pigmented reticular cells in the bone marrow: report of two cases with histochemical studies. Blood, 1959. 14(2): p. 162-9.

311. Li, W., et al., Murine Hermansky-Pudlak syndrome genes: regulators of lysosome-related organelles. Bioessays, 2004. 26(6): p. 616-28.

312. Wei, M.L., Hermansky-Pudlak syndrome: a disease of protein trafficking and organelle function. Pigment Cell Res, 2006. 19(1): p. 19-42.

313. Sanchez-Guiu, I., et al., Hermansky-Pudlak syndrome. Overview of clinical and molecular features and case report of a new HPS-1 variant. Hamostaseologie, 2014. 34(4): p. 301-9.

314. Kelil, T., et al., Hermansky-pudlak syndrome complicated by pulmonary fibrosis: radiologic-pathologic correlation and review of pulmonary complications. J Clin Imaging Sci, 2014. 4: p. 59. 
315. Witkop, C.J., et al., Albinism and Hermansky-Pudlak syndrome in Puerto Rico. Bol Asoc Med P R, 1990. 82(8): p. 333-9.

316. Anikster, Y., et al., Mutation of a new gene causes a unique form of Hermansky-Pudlak syndrome in a genetic isolate of central Puerto Rico. Nat Genet, 2001. 28(4): p. 376-80.

317. Carmona-Rivera, C., et al., Clinical, molecular, and cellular features of non-Puerto Rican Hermansky-Pudlak syndrome patients of Hispanic descent. J Invest Dermatol, 2011. 131(12): p. 2394-400.

318. Oh, J., et al., Positional cloning of a gene for Hermansky-Pudlak syndrome, a disorder of cytoplasmic organelles. Nat Genet, 1996. 14(3): p. $300-6$.

319. Hurford, M.T. and C. Sebastiano, Hermansky-pudlak syndrome: report of a case and review of the literature. Int J Clin Exp Pathol, 2008. 1(6): p. $550-4$.

320. Chiang, P.W., et al., The Hermansky-Pudlak syndrome 1 (HPS1) and HPS4 proteins are components of two complexes, BLOC-3 and BLOC-4, involved in the biogenesis of lysosome-related organelles. J Biol Chem, 2003. 278(22): p. 20332-7.

321. Huizing, M., et al., Nonsense mutations in ADTB3A cause complete deficiency of the beta3A subunit of adaptor complex-3 and severe Hermansky-Pudlak syndrome type 2. Pediatr Res, 2002. 51(2): p. 150-8.

322. Carter, B.W., Hermansky-Pudlak syndrome complicated by pulmonary fibrosis. Proc (Bayl Univ Med Cent), 2012. 25(1): p. 76-7. 
323. Wasmeier, C., et al., Rab38 and Rab32 control post-Golgi trafficking of melanogenic enzymes. J Cell Biol, 2006. 175(2): p. 271-81.

324. Mumford, A.D., et al., A review of platelet secretion assays for the diagnosis of inherited platelet secretion disorders. Thromb Haemost, 2015. 114(1): p. 14-25.

325. Ikawa, Y., et al., In vitro functional correction of Hermansky-Pudlak Syndrome type-1 by lentiviral-mediated gene transfer. Mol Genet Metab, 2015. 114(1): p. 62-5.

326. Zhang, L., et al., Rab38 targets to lamellar bodies and normalizes their sizes in lung alveolar type I/ epithelial cells. Am J Physiol Lung Cell Mol Physiol, 2011. 301(4): p. L461-77.

327. Shotelersuk, V. and W.A. Gahl, Hermansky-Pudlak syndrome: models for intracellular vesicle formation. Mol Genet Metab, 1998. 65(2): p. 85-96.

328. Seward, S.L., Jr. and W.A. Gahl, Hermansky-Pudlak syndrome: health care throughout life. Pediatrics, 2013. 132(1): p. 153-60.

329. Gahl, W.A., et al., Effect of pirfenidone on the pulmonary fibrosis of Hermansky-Pudlak syndrome. Mol Genet Metab, 2002. 76(3): p. 234-42.

330. Rouhani, F.N., et al., Alveolar macrophage dysregulation in HermanskyPudlak syndrome type 1. Am J Respir Crit Care Med, 2009. 180(11): p. 1114-21.

331. Inoshima, I., et al., Anti-monocyte chemoattractant protein-1 gene therapy attenuates pulmonary fibrosis in mice. Am J Physiol Lung Cell Mol Physiol, 2004. 286(5): p. L1038-44. 
332. Henderson, N.C., et al., Galectin-3 expression and secretion links macrophages to the promotion of renal fibrosis. Am J Pathol, 2008. 172(2): p. 288-98.

333. Cullinane, A.R., et al., Dysregulation of galectin-3. Implications for Hermansky-Pudlak syndrome pulmonary fibrosis. Am J Respir Cell Mol Biol, 2014. 50(3): p. 605-13.

334. Trimble, A., et al., Circulating fibrocytes as biomarker of prognosis in Hermansky-Pudlak syndrome. Am J Respir Crit Care Med, 2014. 190(12): p. $1395-401$.

335. Swank, R.T., et al., Mouse models of Hermansky Pudlak syndrome: a review. Pigment Cell Res, 1998. 11(2): p. 60-80.

336. Suzuki, T., et al., Hermansky-Pudlak syndrome is caused by mutations in HPS4, the human homolog of the mouse light-ear gene. Nat Genet, 2002. 30(3): p. 321-4.

337. Zhang, Q., et al., Ru2 and Ru encode mouse orthologs of the genes mutated in human Hermansky-Pudlak syndrome types 5 and 6. Nat Genet, 2003. 33(2): p. 145-53.

338. Feng, G.H., et al., Mouse pale ear (ep) is homologous to human Hermansky-Pudlak syndrome and contains a rare 'AT-AC' intron. Human Molecular Genetics, 1997. 6(5): p. 793-797.

339. Gardner, J.M., et al., The mouse pale ear (ep) mutation is the homologue of human Hermansky-Pudlak syndrome. Proceedings of the National 
Academy of Sciences of the United States of America, 1997. 94(17): $p$. 9238-9243.

340. Feng, L., The beta3A subunit gene (Ap3b1) of the AP-3 adaptor complex is altered in the mouse hypopigmentation mutant pearl, a model for Hermansky- Pudlak syndrome and night blindness. Human Molecular Genetics, 1999. 8(2): p. 323-330.

341. Suzuki, T., et al., The gene mutated in cocoa mice, carrying a defect of organelle biogenesis, is a homologue of the human Hermansky-Pudlak syndrome-3 gene. Genomics, 2001. 78(1-2): p. 30-7.

342. Tang, X., et al., Lung pathology of pale ear mouse (model of HermanskyPudlak syndrome 1) and beige mouse (model of Chediak-Higashi syndrome): severity of giant lamellar body degeneration of type II pneumocytes correlates with interstitial inflammation. Pathol Int, 2005. 55(3): p. 137-43.

343. Young, L.R., et al., Susceptibility of Hermansky-Pudlak mice to bleomycininduced type I/ cell apoptosis and fibrosis. Am J Respir Cell Mol Biol, 2007. 37(1): p. $67-74$.

344. Young, L.R., et al., Lung-restricted macrophage activation in the pearl mouse model of Hermansky-Pudlak syndrome. J Immunol, 2006. 176(7): p. 4361-8.

345. Lyerla, T.A., et al., Aberrant lung structure, composition, and function in a murine model of Hermansky-Pudlak syndrome. Am J Physiol Lung Cell Mol Physiol, 2003. 285(3): p. L643-53. 
346. Mahavadi, P., et al., Epithelial stress and apoptosis underlie HermanskyPudlak syndrome-associated interstitial pneumonia. Am J Respir Crit Care Med, 2010. 182(2): p. 207-19.

347. Young, L.R., et al., The alveolar epithelium determines susceptibility to lung fibrosis in Hermansky-Pudlak syndrome. Am J Respir Crit Care Med, 2012. 186(10): p. 1014-24.

348. Zhou, Y., et al., Chitinase 3-like-1 and its receptors in Hermansky-Pudlak syndrome-associated lung disease. J Clin Invest, 2015. 125(8): p. 317892.

349. Ahuja, S., et al., MAP1LC3B overexpression protects against HermanskyPudlak syndrome type-1-induced defective autophagy in vitro. Am J Physiol Lung Cell Mol Physiol, 2016. 310(6): p. L519-31.

350. Rapaport, S.I., Preoperative hemostatic evaluation: which tests, if any? Blood, 1983. 61(2): p. 229-31.

351. Witkop, C.J., et al., Reliability of absent platelet dense bodies as a diagnostic criterion for Hermansky-Pudlak syndrome. Am J Hematol, 1987. 26(4): p. 305-11.

352. Zhou, L. and A.H. Schmaier, Platelet aggregation testing in platelet-rich plasma: description of procedures with the aim to develop standards in the field. Am J Clin Pathol, 2005. 123(2): p. 172-83.

353. Chakradhar, S., Insurance companies are slow to cover next-generation sequencing. Nat Med, 2015. 21(3): p. 204-5. 
354. Avila, N.A., et al., Hermansky-Pudlak syndrome: radiography and CT of the chest compared with pulmonary function tests and genetic studies. AJR Am J Roentgenol, 2002. 179(4): p. 887-92.

355. Brantly, M., et al., Pulmonary Function and High-Resolution CT Findings in Patients With an Inherited Form of Pulmonary Fibrosis, HermanskyPudlak Syndrome, Due to Mutations in HPS-1. Chest, 2000. 117(1): p. 129-136.

356. Leitman, B.S., et al., The Hermansky-Pudlak syndrome: radiographic features. Can Assoc Radiol J, 1986. 37(1): p. 42-5.

357. Lederer, D.J., et al., Successful bilateral lung transplantation for pulmonary fibrosis associated with the Hermansky-Pudlak syndrome. J Heart Lung Transplant, 2005. 24(10): p. 1697-9.

358. Tomczyk, S., et al., Use of 13-valent pneumococcal conjugate vaccine and 23-valent pneumococcal polysaccharide vaccine among adults aged >/=65 years: recommendations of the Advisory Committee on Immunization Practices (ACIP). MMWR Morb Mortal Wkly Rep, 2014. 63(37): p. 822-5.

359. O'Brien, K., et al., Pirfenidone for the treatment of Hermansky-Pudlak syndrome pulmonary fibrosis. Mol Genet Metab, 2011. 103(2): p. 128-34.

360. Poole, L.G. and G.E. Arteel, Transitional Remodeling of the Hepatic Extracellular Matrix in Alcohol-Induced Liver Injury. Biomed Res Int, 2016. 2016: p. 3162670. 
361. Leung, J., et al., The Role of Aging in Idiopathic Pulmonary Fibrosis. Lung, 2015. 193(4): p. 605-10.

362. Yan Han, e.a., Nicotine, an anti-inflammation molecule. Inflamm Cell Signal, 2014. 1(e155).

363. Gracia, M.C., Exposure to nicotine is probably a major cause of inflammatory diseases among non-smokers. Med Hypotheses, 2005. 65(2): p. 253-8.

364. Behr, J., et al., Antioxidative and clinical effects of high-dose Nacetylcysteine in fibrosing alveolitis. Adjunctive therapy to maintenance immunosuppression. Am J Respir Crit Care Med, 1997. 156(6): p. 1897901.

365. Demedts, M., et al., High-dose acetylcysteine in idiopathic pulmonary fibrosis. N Engl J Med, 2005. 353(21): p. 2229-42.

366. Noth, I., et al., Genetic variants associated with idiopathic pulmonary fibrosis susceptibility and mortality: a genome-wide association study. Lancet Respir Med, 2013. 1(4): p. 309-17.

367. Benam, Kambez H., et al., Matched-Comparative Modeling of Normal and Diseased Human Airway Responses Using a Microengineered Breathing Lung Chip. Cell Systems, 2016. 3(5): p. 456-466.e4.

368. Guo, H., J.B. Callaway, and J.P. Ting, Inflammasomes: mechanism of action, role in disease, and therapeutics. Nat Med, 2015. 21(7): p. 677-87.

369. Hosseinian, N., et al., The role of the NLRP3 inflammasome in pulmonary diseases. Ther Adv Respir Dis, 2015. 9(4): p. 188-97. 
370. El Azreq, M.A., et al., alpha2beta1 integrin regulates Th17 cell activity and its neutralization decreases the severity of collagen-induced arthritis. $\mathrm{J}$ Immunol, 2013. 191(12): p. 5941-50.

371. Nissinen, L., et al., Sulfonamide inhibitors of alpha2beta1 integrin reveal the essential role of collagen receptors in in vivo models of inflammation. Pharmacol Res Perspect, 2015. 3(3): p. e00146.

372. Peters, M.A., et al., The loss of alpha2beta1 integrin suppresses joint inflammation and cartilage destruction in mouse models of rheumatoid arthritis. Arthritis Rheum, 2012. 64(5): p. 1359-68.

373. Peng, Q., et al., Multiple beta 1 integrins mediate enhancement of human airway smooth muscle cytokine secretion by fibronectin and type I collagen. J Immunol, 2005. 174(4): p. 2258-64.

374. Jun, H.K., et al., Integrin alpha5beta1 activates the NLRP3 inflammasome by direct interaction with a bacterial surface protein. Immunity, 2012. 36(5): p. 755-68.

375. Jones, D.P. and Y. Liang, Measuring the poise of thiol/disulfide couples in vivo. Free Radic Biol Med, 2009. 47(10): p. 1329-38.

376. Moriarty-Craige, S.E. and D.P. Jones, Extracellular thiols and thiol/disulfide redox in metabolism. Annu Rev Nutr, 2004. 24: p. 481-509.

377. Ritzenthaler, J.D., et al., Nicotinic acetylcholine receptors are sensors for ethanol in lung fibroblasts. Alcohol Clin Exp Res, 2013. 37(6): p. 914-23.

378. Go, Y.M. and D.P. Jones, Cysteine/cystine redox signaling in cardiovascular disease. Free Radic Biol Med, 2011. 50(4): p. 495-509. 
379. Moriarty, S.E., et al., Oxidation of glutathione and cysteine in human plasma associated with smoking. Free Radic Biol Med, 2003. 35(12): p. $1582-8$.

380. lyer, S.S., et al., Oxidation of extracellular cysteine/cystine redox state in bleomycin-induced lung fibrosis. Am J Physiol Lung Cell Mol Physiol, 2009. 296(1): p. L37-45.

381. Ramirez, A., et al., Extracellular cysteine/cystine redox potential controls lung fibroblast proliferation and matrix expression through upregulation of transforming growth factor-beta. Am J Physiol Lung Cell Mol Physiol, 2007. 293(4): p. L972-81.

382. Benam, Kambez H., et al., Matched-Comparative Modeling of Normal and Diseased Human Airway Responses Using a Microengineered Breathing Lung Chip. Cell Systems, 2016.

383. Alexandrov, L.B., et al., Mutational signatures associated with tobacco smoking in human cancer. Science, 2016. 354(6312): p. 618-622.

384. Samet, J.M., Does idiopathic pulmonary fibrosis increase lung cancer risk? Am J Respir Crit Care Med, 2000. 161(1): p. 1-2.

385. Vicary, G.W. and J. Roman, Targeting the Mammalian Target of Rapamycin in Lung Cancer. The American Journal of the Medical Sciences. 352(5): p. 507-516. 


\section{ABBREVIATIONS}

\begin{tabular}{ll} 
AEC & Alveolar Epithelial Cells \\
ELF & Epithelial Lining Fluid \\
EMT & Epithelial-Mesenchymal Transition \\
FGF & Fibroblast Growth Factor \\
HPS & Hermansky-Pudlak Syndrome \\
HPS-PF & Hermansky-Pudlak Syndrome Associated Pulmonary Fibrosis \\
IIP & Idiopathic Interstitial Pneumonia \\
ILD & Interstitial Lung Disease \\
IPF & Idiopathic Pulmonary Fibrosis \\
MAPK & Mitogen-Activated Protein Kinase \\
PDGR & Platelet-Derived Growth Factor Receptor \\
ROS & Reactive Oxygen Species \\
SOD & Superoxide Dismutase \\
TGF- $\beta$ & Transforming Growth Factor- $\beta$ \\
TNF- $\alpha$ & Tumor Necrosis Factor- $\alpha$ \\
VEGF & Vascular Endothelial Growth Factor \\
\hline
\end{tabular}




\section{CURRICULUM VITA}

NAME:

ADDRESS:

DOB:

EDUCATION \& TRAINING:
Glenn Ward Vicary

1038 Brent Street Unit 104

Louisville, KY 40204

Oak Ridge, Tennessee, USA - November 9, 1987

B.S., Biology Minoring in Chemistry and Business Tusculum College 2006-2010

M.S., Pharmacology and Toxicology University of Louisville 2010-2013

Entrepreneurship Academy University of Louisville 2014

Ph.D., Pharmacology and Toxicology University of Louisville 2013-Current 
COMMUNITY SERVICE:

2015 - Volunteer. YMCA Healthy Kids Day

2012-2013 - Judge. Manual High School Science Fair

2013 - Coach. Upward Children's Soccer Program

2012 - Mentor. South Oldham High School Science Class

2011 - Judge. St. Patrick Catholic School Science Fair.

EXTRACURRICULAR:

2015 - Present - Research Intern, Apellis Pharmaceuticals,

Louisville, KY USA

2015 - 2016 - Research Intern, Revon Systems Inc.,

Louisville, KY USA

2011 - Present - Vice-President. Brent Park Condominium

Board

2011 - Present - Team Captain. Louisville Metro Parks

Kickball

2015 - Participant. StartUpLouisville

GRANT SUPPORT:

$\mathrm{NIH}$ Science-funded Training Program in Environment Health Sciences, T32-ES011564. The Role of Nicotinic Acetylcholine Receptors in Lung Injury and Repair. 
Vicary, GW., Roman, J., Pulmonary Fibrosis in Hermansky-Pudlak Syndrome - Insight into the pathogenesis of fibrosing lung disorders. Annals of American Thoracic Society, 2016. 13(10): p. 1839-1846

Vicary, GW., Roman, J., mTOR in Lung Cancer. American Journal of Medical Science, 2016. 352(5): p. 507-516

Vicary, GW. Torrez-González, E., Panchabhai, T., Ritzenthaler, JD., Nicotine stimulates collagen type I expression in lung via $\alpha 7$ nicotinic acetylcholine receptors. Respiratory Research. (Submitted)

Fan, Y., Ritzenthaler, JD., Zhang, W., Vicary, GW., \& Roman, J., CRKL stimulates epithelial-mesenchymal transition in non-small cell lung carcinoma cells via activation of ERK and induction of integrins. Thoracic Cancer. (Submitted)

Vicary, GW., Ellis. B. Does Exposure to the Hanford Tanks Affect Absence Rates of Employees? Oak Ridge Associated Universities Internal Report. 2009

Vicary, GW., Furmanek, S. Mattingly, B., Barber, C., Wiemken, T., Persaud, A., Guinn, B., Roman, J., Perez,

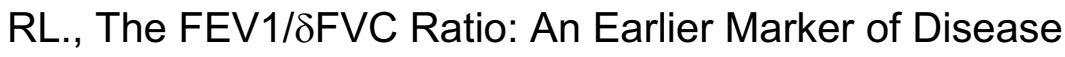
Progression in Idiopathic Pulmonary Fibrosis. (In Preparation)

Vicary, GW. Ritzenthaler, JD., Torrez-González, E., Roman, J., The role of nicotine in bleomycin-induced death and the potential role of superoxide dismutase. (In Preparation)

Vicary, GW. Ritzenthaler, JD., Torrez-González, E., Soucy P., Roman, J., The role of nicotine in bleomycin-induced pulmonary fibrosis. (In Preparation) 
POSTER

PRESENTATION:

Vicary, GW., Furmanek, S. Mattingly, B., Barber, C., Wiemken, T., Persaud, A., Guinn, B., Roman, J., Perez, RL., The FEV1/8FVC ratio: An Earlier Marker of Disease Progression in Idiopathic Pulmonary Fibrosis, American Thoracic Society National Meeting, Louisville KY, USA, 2017. Research!Louisville, Louisville KY, USA, 2016.

Vicary, GW., Ritzenthaler, JD., Torrez-González, E., Soucy P., Roman, J. Decellularized Normal and Fibrotic Murine Lung as a Culture System for Ex Vivo Investigation of CellMatrix Interactions. American Thoracic Society National Meeting, San Diego, CA, USA, 2015, Research!Louisville, Louisville KY, USA, 2015.

Vicary, GW., Ritzenthaler, JD., Torrez-González, E., Roman, J. Nicotine in Bleomycin-Induced Lung Injury: Protector or Cover-up?. American Thoracic Society National Meeting, San Diego, CA, USA, 2014, Research!Louisville, Louisville, KY, USA, 2014.

Vicary, GW., Ritzenthaler, JD., Torrez-González, E., Roman, J. Nicotine Induces a Collagen Rich Transitional Matrix: Roles of $\alpha 7$ nAChRs, MEK-1/Erk pathways, and Integrin Receptors. American Thoracic Society National Meeting, Philadelphia, PA, USA, 2013.

Vicary, GW., Ritzenthaler, JD., Torrez-González, E., Roman, J. Protective Role of Nicotine in Bleomycin-Induced Lung Injury and Development of Acellular Lungs.

Research!Louisville, Louisville, KY, USA, 2013.

Fan, Yu, Ritzenthaler, JD., Zhang, W., Vicary, GW., Roman, J. CRKL Stimulates Epithelial-Mesenchymal Transition in Non-Small Cell Lung Carcinoma Cells via activation of ERK and Induction of $\alpha 9 \beta 1$ integrin. American Thoracic Society National Meeting, San Francisco, CA, USA, 2012.

Vicary, GW., Ritzenthaler, JD., Torrez-González, E., 
Roman, J. Nicotine Stimulates Lung Fibroblasts to Produce a Type I Collagen-Matrix with Pro-Inflammatory Properties:

Role of $\alpha 7$ Nicotinic Acetylcholine Receptors and Mek1/Erk Pathways. Research!Louisville, Louisville, KY, USA, 2012.

Vicary, GW., Torrez-González, E., Panchabhai, T., Ritzenthaler, JD., Roman, J. Nicotine Induces Collagen Type I Expression in Lung: Role of Fibroblasts and Implications for Tobacco-Related Inflammation. American Thoracic Society National Meeting, Denver, CO, USA, 2011. 